\title{
WestVirginiaUniversity
}

THE RESEARCH REPOSITORY @ WVU

Graduate Theses, Dissertations, and Problem Reports

2005

\section{Dynamics and mechanism studies of nonlinear chemical systems}

Tabitha R. Chigwada-Razunguzwa

West Virginia University

Follow this and additional works at: https://researchrepository.wvu.edu/etd

\section{Recommended Citation}

Chigwada-Razunguzwa, Tabitha R., "Dynamics and mechanism studies of nonlinear chemical systems" (2005). Graduate Theses, Dissertations, and Problem Reports. 4141.

https://researchrepository.wvu.edu/etd/4141

This Dissertation is protected by copyright and/or related rights. It has been brought to you by the The Research Repository @ WVU with permission from the rights-holder(s). You are free to use this Dissertation in any way that is permitted by the copyright and related rights legislation that applies to your use. For other uses you must obtain permission from the rights-holder(s) directly, unless additional rights are indicated by a Creative Commons license in the record and/ or on the work itself. This Dissertation has been accepted for inclusion in WVU Graduate Theses, Dissertations, and Problem Reports collection by an authorized administrator of The Research Repository @ WVU.

For more information, please contact researchrepository@mail.wvu.edu. 


\title{
Dynamics and Mechanism Studies of Nonlinear Chemical Systems
}

Tabitha R. Chigwada-Razunguzwa

Dissertation submitted to the Eberly College of Arts and Sciences at West Virginia University in partial fulfillment of the requirements for the Degree of

\author{
Doctor of Philosophy \\ in \\ Chemistry
}

Kenneth Showalter, Ph.D., Co-Chair

Reuben H. Simoyi, Ph.D., Co-Chair

John H. Penn, Ph.D.

Terry Gullion, Ph.D.

Boyd Edwards, Ph.D.

C. Eugene Bennett Department of Chemistry

Morgantown, West Virginia

2005

Keywords: sulfur metabolism, S-oxygenation, thiourea, thiocarbamide, trimethylthiourea, phenylthiourea, tetramethylthiourea, aminoethanethiolsulfuric acid, oregonator model, resonance, spatiotemporal pattern, excitable media, photosensitive BZ reaction, chemical waves, peroxidase-oxidase reaction, BFSO model, 1983 Olsen model, NADH, oxygen 


\section{ABSTRACT \\ Dynamics and mechanism studies of nonlinear chemical systems \\ Tabitha R. Chigwada-Razunguzwa}

The kinetics and mechanisms of oxidation of selected thiocarbamides (tetramethylthiourea, trimethylthiourea, phenylthiourea, and 2-aminoethanethiolsulfuric acid) by chlorite in aqueous acidic media are investigated using UV/Vis, NMR, Stopped-flow techniques, and qualitative analysis. The reactions were extremely complex, with reaction dynamics strongly influenced by the $\mathrm{pH}$ of the reaction medium and formation of stable intermediates (sulfonic acids). Results revealed that oxidations of substituted thioureas do not always proceed via a stepwise oxidation of the sulfur center. Instead, reactions occurred in two stages: S-oxygenation of the sulfur center to yield the sulfinic acid, which then reacts in the second phase predominantly through an initial hydrolysis to produce a urea-type residue and the sulfoxylate anion. The sulfoxylate anion, a highly reducing species, is then rapidly oxidized to sulfate.

Experimental and numerical studies of local periodic forcing on an excitable Belousov-Zhabotinsky (BZ) medium in a thin gel layer are reported. Rather than the traditional suprathreshold perturbations giving rise to a local oscillatory state, waves were initiated in an excitable system via localized small amplitude variations in light intensity, without crossing into the oscillatory regime of the autonomous system. Initiation of waves in the initially quiescent medium was possible when the frequency of the sinusoidal perturbation was suitably tuned to that of the autonomous system. The region 
in phase space where wave initiation was possible depended on the parameter values of the perturbation, namely forcing frequency and forcing amplitude, and on the inherent properties of the autonomous system. Resonance patterns are found by relating the period between two waves to the period of the sinusoidal perturbation.

Experimental and theoretical studies of the peroxidase-oxidase (PO) reaction are reviewed. Numerical investigations into the initiation of trigger waves in an oscillatory one-dimensional PO reaction-diffusion system are presented. Trigger waves are initiated in the oscillatory system via localized perturbations in the concentration of one of the variables using the extended BFSO model. The chemical waves traveled with a sharp front and were not able to penetrate barriers to diffusion, which are properties characteristic of trigger waves. 
To my parents and husband for believing in me always. 


\section{ACKNOWLEDGEMENTS}

First and foremost I would like to thank my two graduate advisors and mentors, Dr. Reuben H. Simoyi and Dr. Kenneth Showalter, for their guidance and invaluable support throughout my studies at WVU. They introduced me into the fascinating and interesting fields of chemical kinetics and nonlinear dynamics.

I would like to express my gratitude to Drs. Florin Chirila and Mark Tinsley for insightful and stimulating discussions on the peroxidase-oxidase and resonance induced chemical waves projects. I am also indepted to Drs. Jianxia Cui and Eugene Mihaliuk for helpful suggestions on the resonance induced chemical waves project. Special thanks go

to Dr. Rotimi Olojo for his invaluable contributions on the S-oxygenation of selected thiocarbamides project. My deepest appreciation is extended to my research committee members, Drs. Terry Gullion, John Penn and Boyd Edwards, for their time, constructive criticisms and invaluable help afforded me in getting this work to this stage.

I would also like to thank my colleagues and friends, Edward Chikwana, Olufunke M. Olagunju, Mitsuhiro Murata, Aaron Steele, Yongjun Li, Zhaoyang Huang, Lauren Boyle, On-Uma Kheowan, and Shi Zhong. I would also like to express my sincere gratitude to my parents, sisters, and brothers for their enormous support and encouragement throughout my educational and professional pursuit over the years.

Finally, I am very grateful to my husband and best friend, Trust Razunguzwa, for his endless support, patience, and understanding all through my educational and professional pursuit over the years. 


\section{TABLE OF CONTENTS}

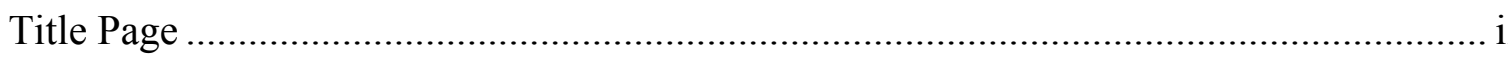

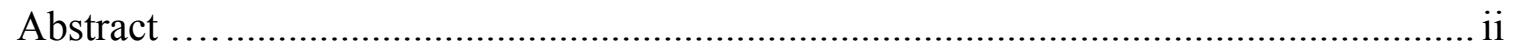

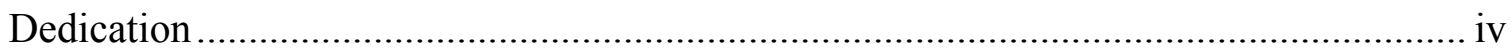

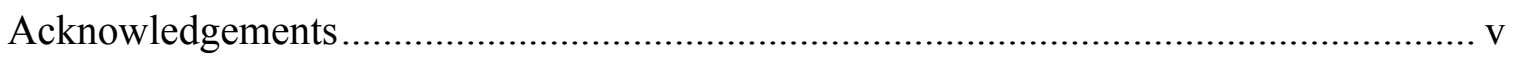

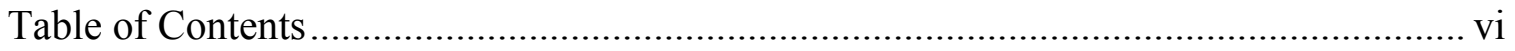

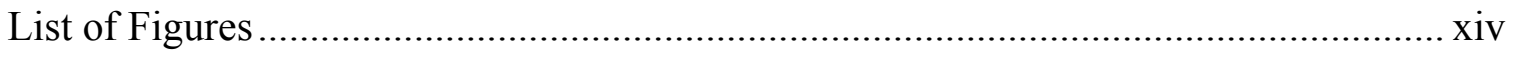

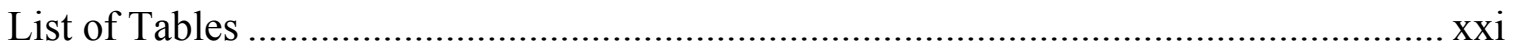

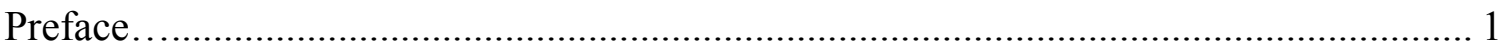

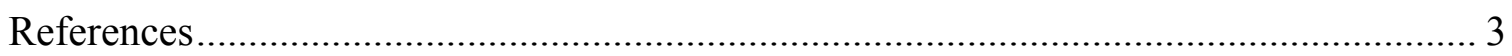

\section{PART I}

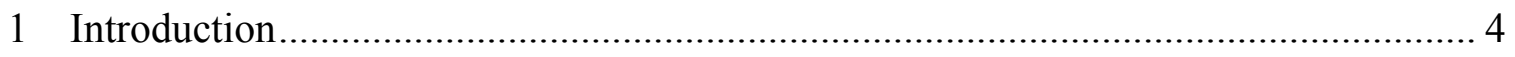

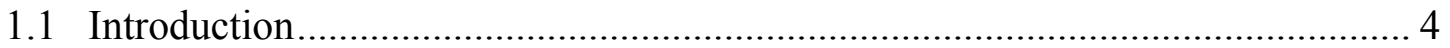

1.1.1 Sulfur-Oxygen Compounds ................................................................. 7

1.1.2 Metabolic Activation of S-atom via S-oxygenation .................................. 9

1.1.2.1 Enzymes involved in S-oxygenation ......................................... 10

1.3.1 Thiocarbamides $\left(\mathrm{R}^{1} \mathrm{R}^{2} \mathrm{NC}(=\mathrm{S}) \mathrm{NHR}^{3} \leftrightarrow \mathrm{R}^{1} \mathrm{R}^{2} \mathrm{C}(\mathrm{SH})=\mathrm{NR}^{3}\right) \ldots \ldots \ldots \ldots \ldots \ldots \ldots . .11$

1.3.1.1 Toxicity of Thiocarbamides ....................................................... 13

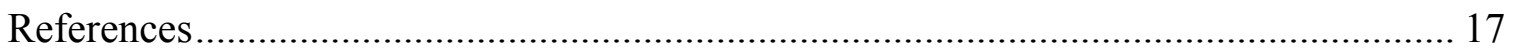

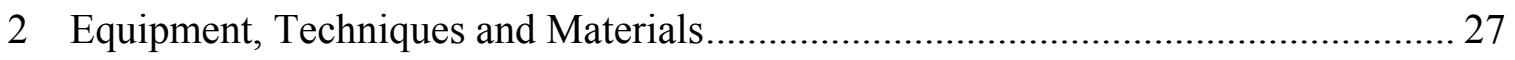

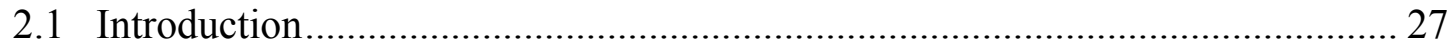

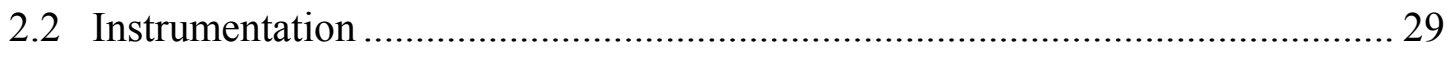

2.2.1 Conventional UV/Vis Spectrophotometry .............................................. 29 
2.2.2 Stopped-Flow Spectrophotometry ……………….................................. 30

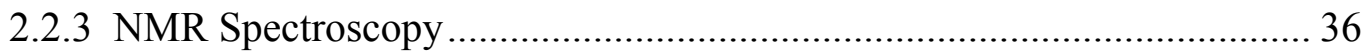

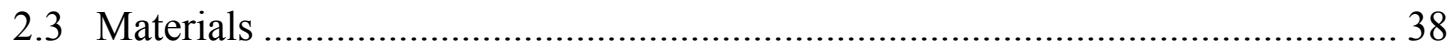

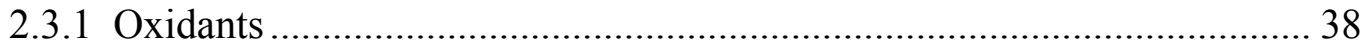

2.3.1.1 Preparation of Chlorine Dioxide.................................................. 39

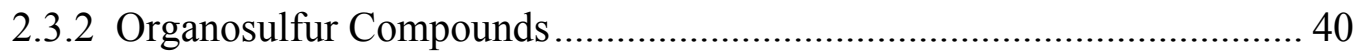

2.3.2.1 Synthesis of Phenylthiourea Dioxide .......................................... 40

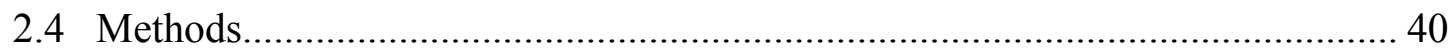

2.4.1 Reactant and Product Analysis .................................................................. 41

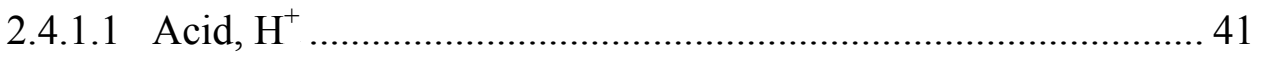

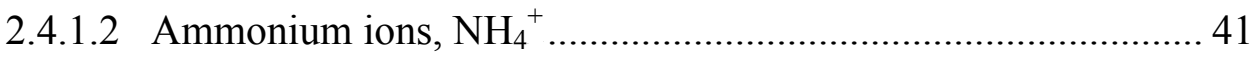

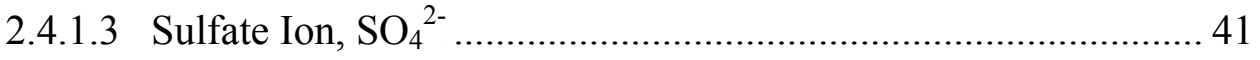

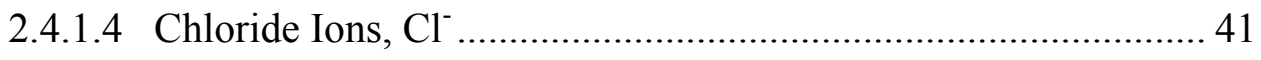

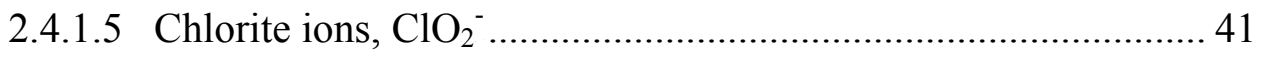

2.4.1.6 Tests for Adventitious Metal Ion Catalysis ................................ 42

2.4.2 Stoichiometric Determinations ............................................................. 42

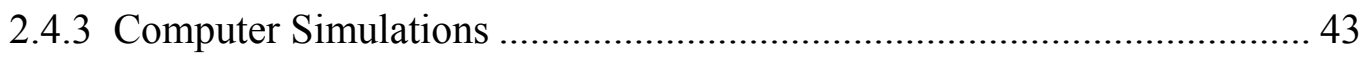

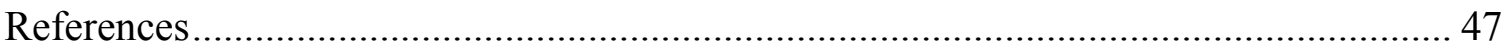

3 Oxidation of TTTU by Chlorite in Acidic Media .................................................... 51

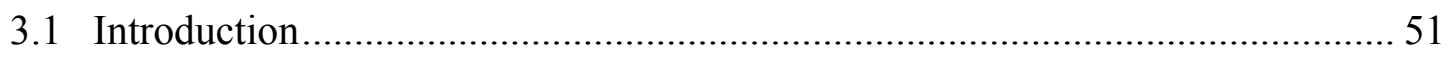

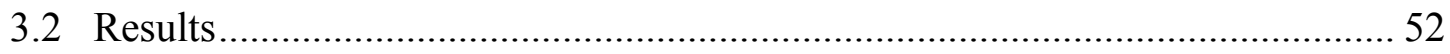

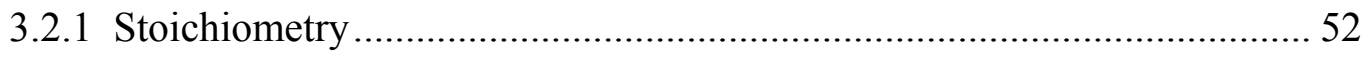

3.2.2 Reactant and Product Analysis .................................................................. 54 
3.2.2.1 Chlorite Dependence.................................................................... 55

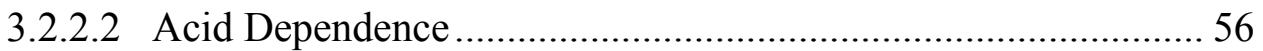

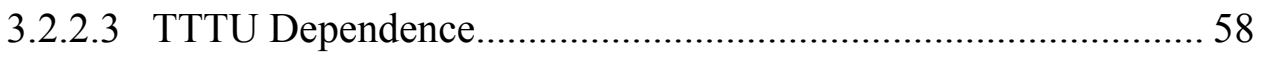

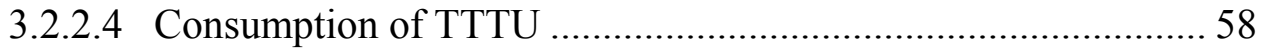

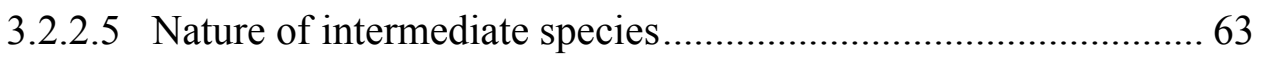

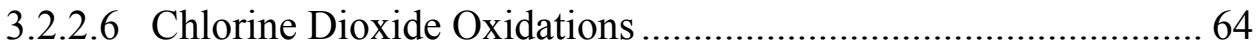

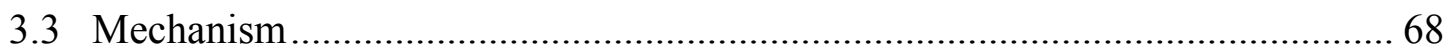

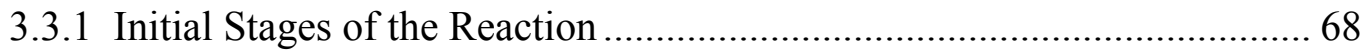

3.3.2 Chlorine Dioxide Formation .................................................................... 71

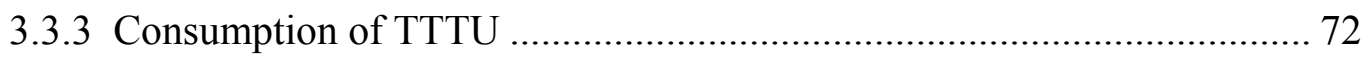

3.3.4 Chlorine Dioxide Consumption ............................................................. 73

3.3.5 Overall Reaction Mechanism and Computer Modeling ............................ 75

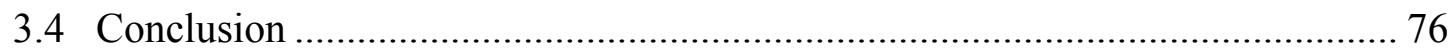

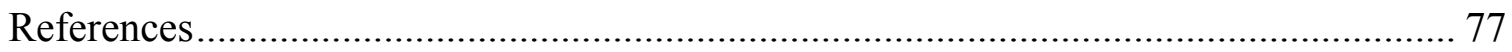

4 Oxidation of TMTU by Chlorite and Chlorine Dioxide ........................................... 79

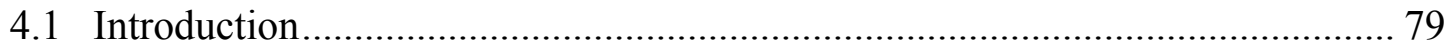

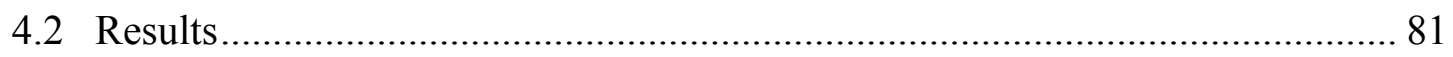

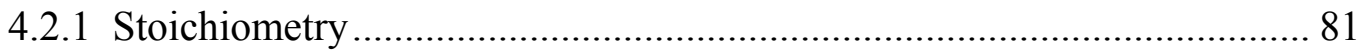

4.2.2 Reaction Dynamics ......................................................................... 82

4.2.2.1 Chlorite Dependence................................................................ 84

4.2.2.2 Acid Dependence .................................................................... 85

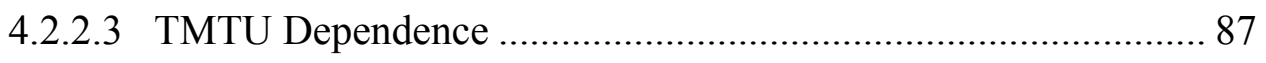

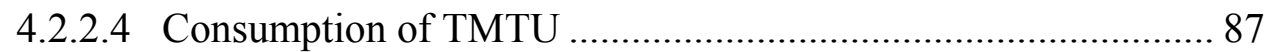




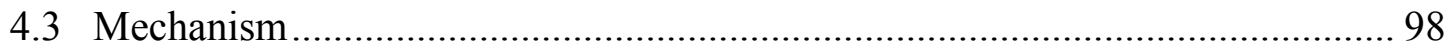

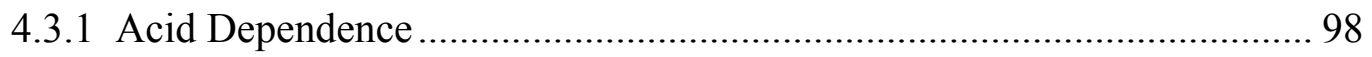

4.3.2 Further Oxidation of TMTU ................................................................. 103

4.3.3 Formation of Products......................................................................... 103

4.3.3.1 Formation of Chlorine Dioxide................................................ 107

4.3.4 Chlorine Dioxide Consumption ............................................................. 108

4.3.5 Experimental Evaluation of some Rate Constants .................................. 109

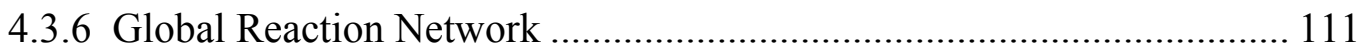

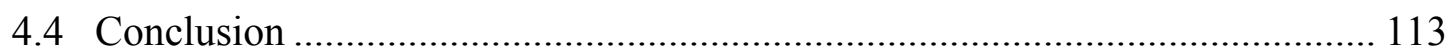

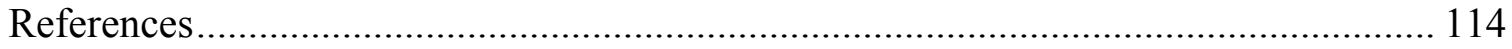

5 Oxidation of PTU by Chlorite in Acidic Media........................................................ 118

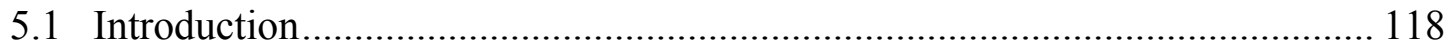

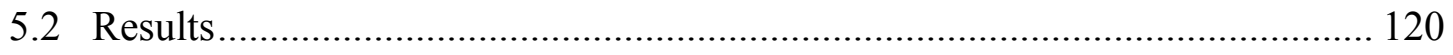

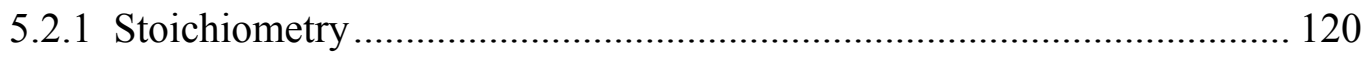

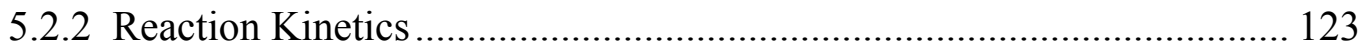

5.2.2.1 Chlorite Dependence.............................................................. 123

5.2.2.2 Acid Dependence .................................................................... 126

5.2.2.3 The Chlorite-Phenylthiourea Ratio .......................................... 128

5.2.2.4 Chlorine Dioxide Formation .................................................... 130

5.2.2.5 Oxidations by Chlorine Dioxide ................................................ 132

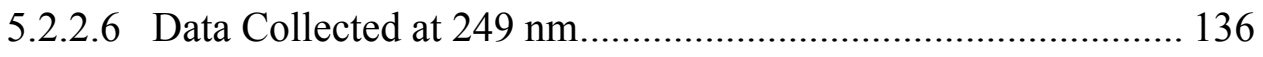

5.2.2.7 Chlorine Dioxide Oxidations Monitored at $249 \mathrm{~nm}$.................. 141 


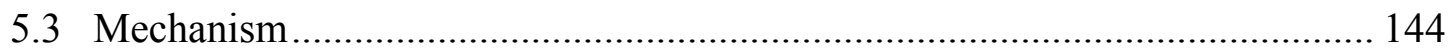

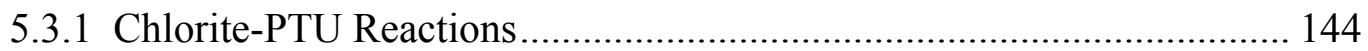

5.3.1.1 Evaluation of Kinetic Parameters $k_{1}$ and $k_{2} \ldots \ldots \ldots \ldots \ldots \ldots \ldots \ldots \ldots \ldots . \ldots . \ldots . \ldots . \ldots . \ldots 148$

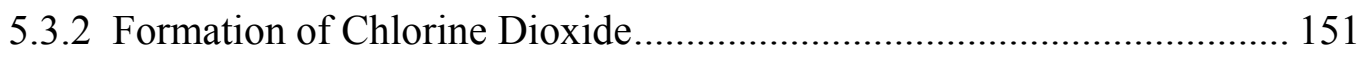

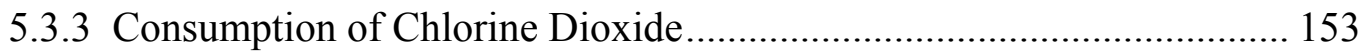

5.3.4 Nonlinear Consumption and Formation of Chlorine Dioxide ................ 154

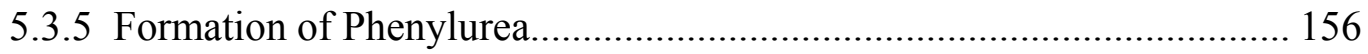

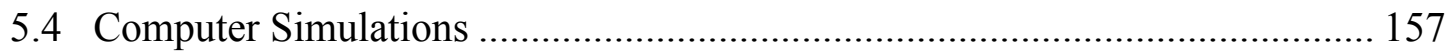

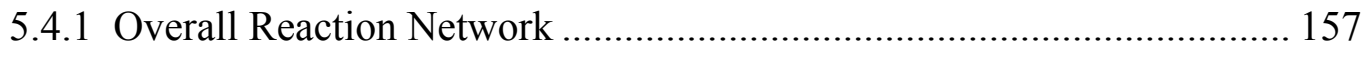

5.4.2 Choice and Establishment of Rate Constants ....................................... 160

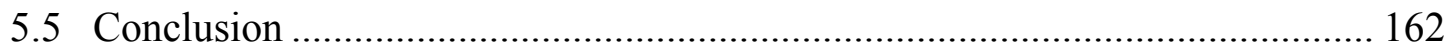

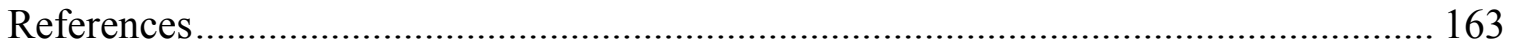

6 Kinetics and Mechanisms of the Oxidation of a Bunte salt AETSA by Chlorite..... 167

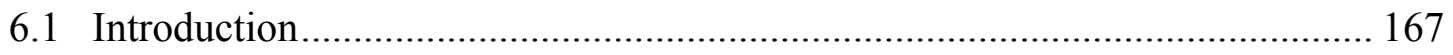

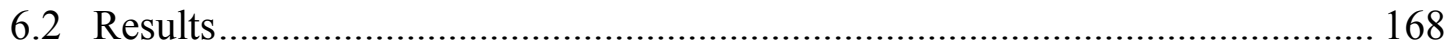

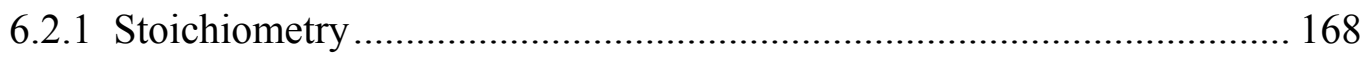

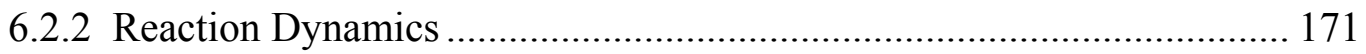

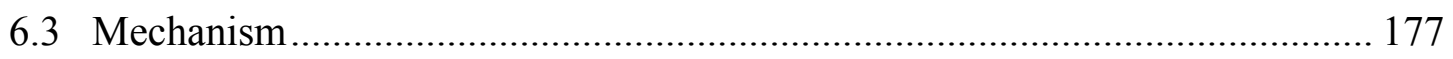

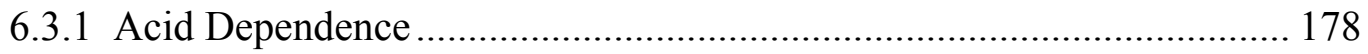

6.3.2 Consumption of AETSA ...................................................................... 180

6.3.3 Reaction of Chlorine Dioxide with AETSA ………………………........ 184

6.3.4 Formation of Chlorine Dioxide.............................................................. 186 
6.3.5 Quantitative Formation of Chlorine Dioxide

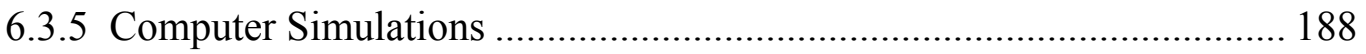

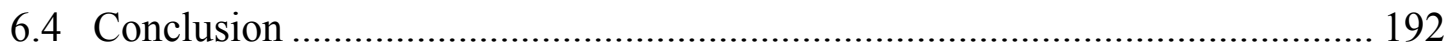

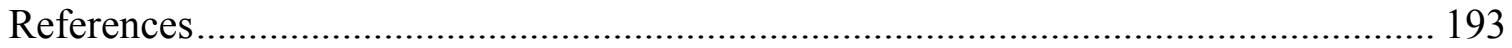

\section{PART II}

7 Resonance Induced Chemical Waves ....................................................................... 197

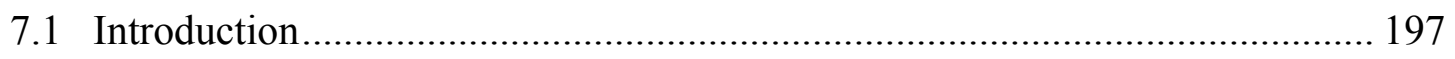

7.1.1 The Belousov-Zhabotinsky (BZ) Reaction.......................................... 200

7.1.1.1 Effect of Light on the Belousov-Zhabotinsky (BZ) Medium ... 201

7.1.1.2 The FKN Mechanism............................................................. 202

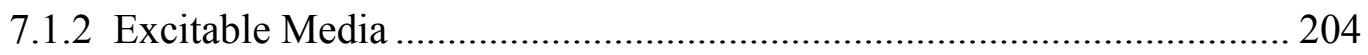

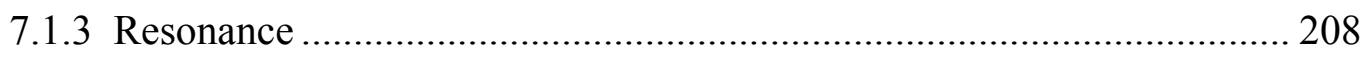

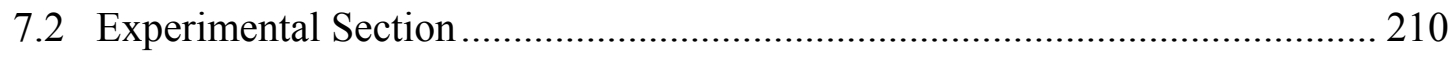

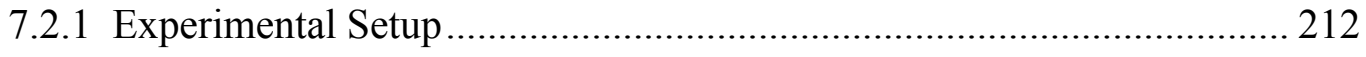

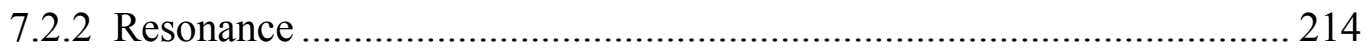

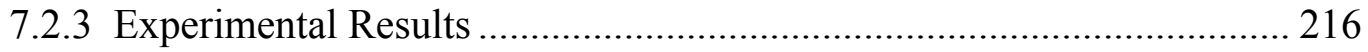

7.3 Numerical Simulations.......................................................................... 220

7.3.1 Resonance Induced Chemical Waves .................................................... 221

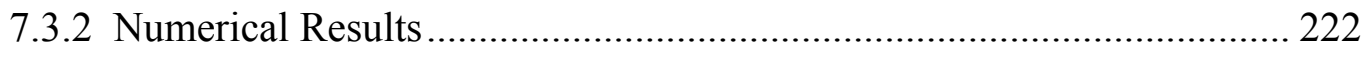

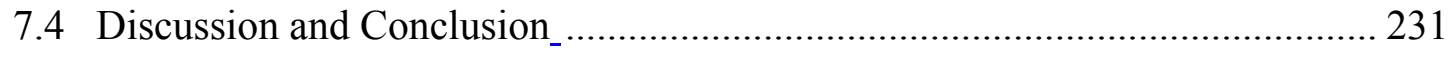

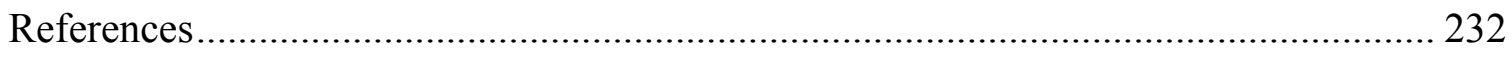

8 Chemical Waves in the Oscillatory Peroxidase-Oxidase Reaction ......................... 239

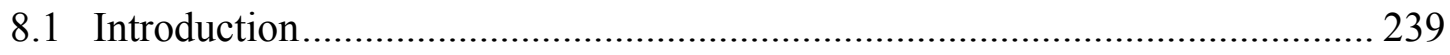




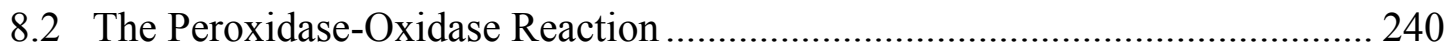

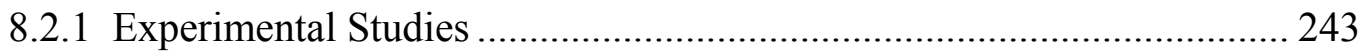

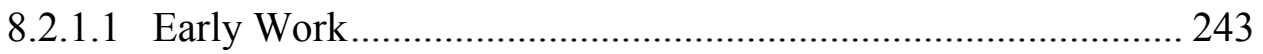

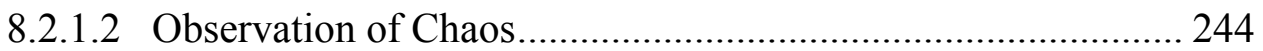

8.2.1.3 The Role of Methylene Blue................................................... 244

8.2.1.4 The Role of 2,4 Dichlorophenol ............................................ 245

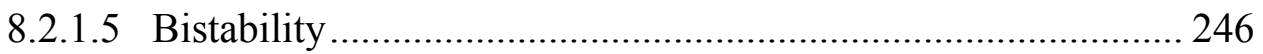

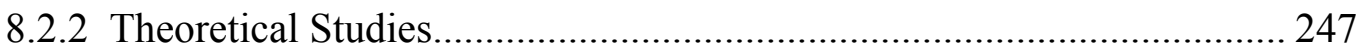

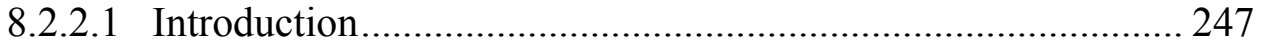

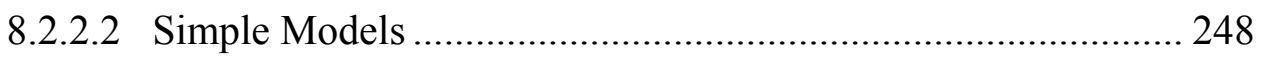

8.2.2.2.1 The Four-Variable Model ......................................... 248

8.2.2.2.1 The Dunford Model ................................................... 249

8.2.2.3 Detailed Models .................................................................... 250

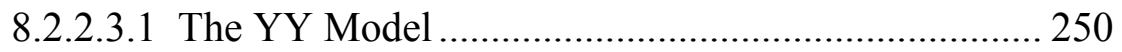

8.2.2.3.2 The FAB Model ...................................................... 250

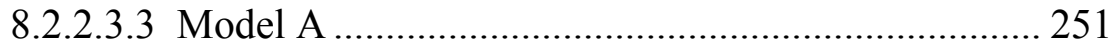

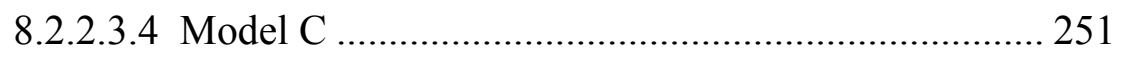

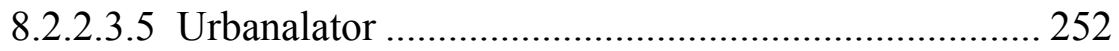

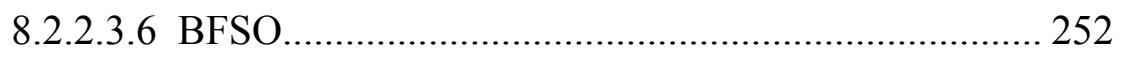

8.3 Chemical Waves In The Peroxidase-Oxidase Reaction.................................... 255

8.3.1 Generation of Chemical Waves in the BFSO-14 Model ........................ 256

8.3.1.1 The Chemical Reaction Model ............................................... 256

8.3.1.2 Reaction-Diffusion Equations for BFSO-14 Model ................. 258 
8.3.1.3 Wave Generation in the One-Dimensional BFSO-14 system... 258

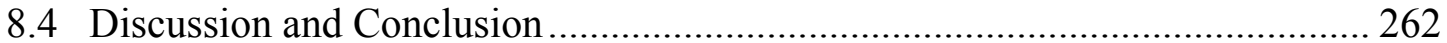

8.2 The Peroxidase-Oxidase Reaction .......................................................... 263

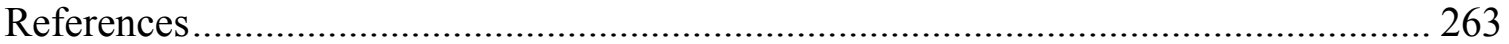




\section{LIST OF FIGURES}

1.1 Active Site of Flavin-Containing Monooxygenase (FMO) ….......................... 10

2.1 Schematic Diagram Showing all Components of the SF61-DX2 Double Mixing Stopped-Flow Fluorimeter 34

2.2 Sample Handling Unit (SHU) Flow Circuit Diagram for the SF-61 DX2 Double Mixing Stopped-Flow System 35

2.3 System Propagation Routine in the Stochastic Algorithm Employed by CKS .... 46

3.1 UV Absorption Spectrums of TTTU, $\mathrm{ClO}_{2}$, and Tetramethylurea ...................... 53

3.2 Absorbance Scans Of TTTU during Oxidation by Chlorite in Aqueous Acidic

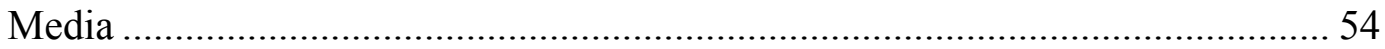

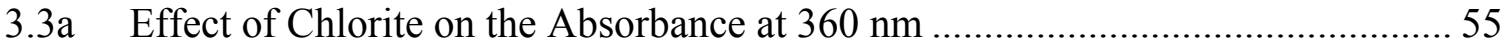

3.3b Plot Of $1 / \mathrm{T}_{\text {ind }}$ Vs. $\left[\mathrm{Clo}_{2}^{-}\right]$Using Stoichiometric Excess Of Chlorite, Showing that an Inverse Relationship Exists for a Long Range of Chlorite Concentrations ..... 56

3.4a Absorbance Traces at $360 \mathrm{~nm}$ Showing Effect of Low Acid Concentrations on the Oxidation of TTTU

3.4b Absorbance traces at $360 \mathrm{~nm}$ Showing Effect of High Acid Concentrations on the Oxidation of TTTU by Chlorite.

3.5 "Peacock-tail type" Traces Derived from Variation of [TTTU] $]_{0}$ in Large Excess of $\left[\mathrm{ClO}_{2}^{-}\right]_{0}$ 58

3.6a Effect of Chlorite on the Depletion of TTTU in its Oxidation by Chlorite Monitored at $248 \mathrm{~nm}$ 59

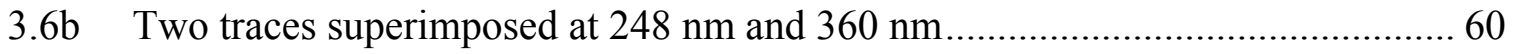


3.6c Effect Of Acid on the Depletion of TTTU in its Oxidation by Chlorite Monitored at $248 \mathrm{~nm}$

3.6d Effect of Acid on the Depletion of TTTU in its Oxidation by Chlorite Monitored at $248 \mathrm{~nm}$ 61

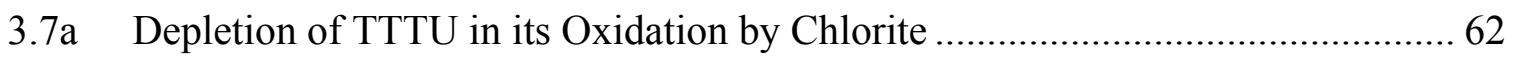

3.7b Effect of Initial TTTU Concentration on the Initial Rate of Consumption of Tetramethylthiourea 63

3.8 Effect of Acid on the Oxidation of TTTU by Chlorine Dioxide 65

3.9a Effect of Chlorine Dioxide in the Depletion of TTTU .........................................6 66

3.9b Expansion of the First Two Seconds of the Absorbance Traces shown in Figure 3.9a. 66

3.10a Effect of Chlorine Dioxide on the Oxidation of TTTU ………………................. 67

3.10b Variation of TTTU in its Oxidation by Chlorine Dioxide under Close to Pseudofirst Order Kinetics Environments

3.11 Effect of Chloride on the Oxidation of TTTU by Chlorine Dioxide .................... 68

4.1 UV Absorption Spectrum of TMTU, $\mathrm{ClO}_{2}$, and Trimethylurea .......................... 81

4.2 Absorbance Scans of TMTU during Oxidation by Chlorite in Aqueous Acidic Medium 83

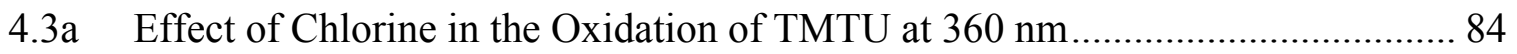

4.3b Plot Of $1 / \mathrm{T}_{\text {ind }}$ Vs. $\left[\mathrm{Clo}_{2}{ }^{-}\right]$Using Stoichiometric Excess Of Chlorite, Showing that an Inverse Relationship Exists for a Long Range of Chlorite Concentrations ..... 85

4.4a Absorbance Traces at $360 \mathrm{~nm}$ Showing Effect of Low Acid Concentrations on the Oxidation of TMTU by Chlorite 86 
4.4b Absorbance Traces at $360 \mathrm{~nm}$ Showing Effect of High Acid Concentrations on the

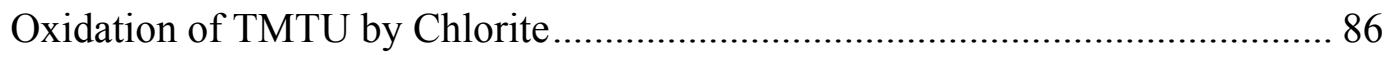

4.5 Effect of Varying TMTU on the Formation of Chlorine Dioxide ........................ 87

4.6a Effect of Acid on the Depletion of TMTU in its Oxidation by Chlorite Monitored

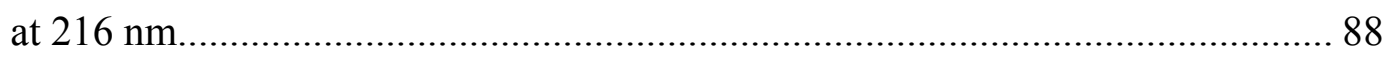

4.6b Effect of Acid on the Depletion of TMTU in its Oxidation by Chlorite Monitored

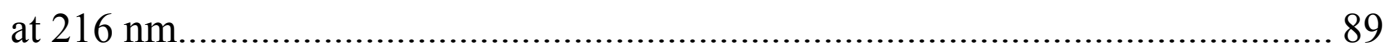

4.6c Effect of Acid on the Depletion of TMTU in its Oxidation by Chlorite Monitored at $216 \mathrm{~nm}$

4.7a Effect of Chlorite on the Depletion of TMTU in its Oxidation by Chlorite

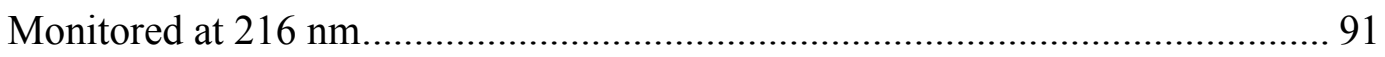

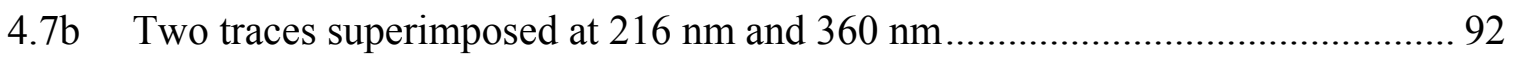

4.8a Depletion of TMTU in its Oxidation by Chlorite ................................................ 93

4.8b Effect of Initial TMTU Concentration on the Initial Rate of Consumption of

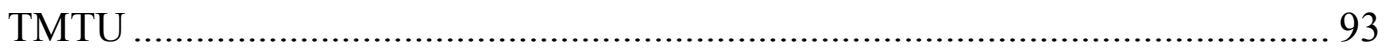

4.9a Oxidation of TMTU by Chlorine Dioxide Monitored at $360 \mathrm{~nm}$......................... 94

4.9b Effect of Chlorine Dioxide in the Depletion of TMTU …………….................... 95

4.10 Effect of Low Acid Concentrations on the Oxidation of TMTU by Chlorine

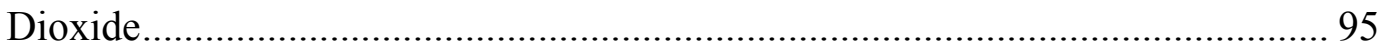

4.11 Effect of Chlorite on the Oxidation of TMTU by Chlorine Dioxide .................... 96

4.12a Chlorine Dioxide Variation in High Excess of TMTU at Intermediate Acid

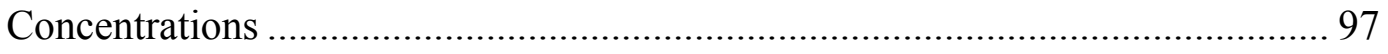


4.12b Pseudo-first Order Kinetics in High Excess of TMTU and High Acid Concentrations

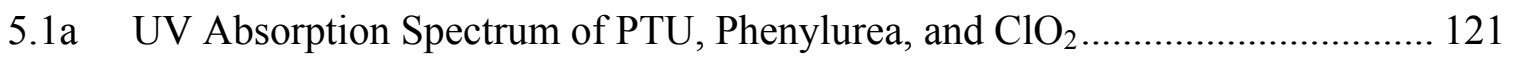

5.1b Spectral Scans of Reagent Solutions Containing Phenylthiourea and Varying Concentrations of Chlorite between $200 \mathrm{~nm}$ and $350 \mathrm{~nm}$.

5.2a General Absorbance Traces at $360 \mathrm{~nm}$ of the Reaction in Excess Chlorite ....... 124

5.2b Effect of Chlorite on the Induction Period...................................................... 125

5.3a Effect of Acid Concentrations on the Reaction Profiles with $\left[\mathrm{ClO}_{2}{ }^{-}\right]_{0} /[\mathrm{PTU}]_{0}=$ 4.0

$5.3 \mathrm{~b}$ Effect of Acid at High Oxidant to Reductant Ratios $\left[\mathrm{ClO}_{2}^{-}\right]_{0} /[\mathrm{PTU}]_{0}=10.0 \ldots . . .127$

$5.3 \mathrm{c}$ Effect of Acid at High Oxidant to Reductant Ratios $\left[\mathrm{ClO}_{2}{ }^{-}\right]_{0} /[\mathrm{PTU}]_{0}=10.0 \ldots . .128$

5.4 Effect of Varying [PTU $]_{0}$ on the formation of Chlorine Dioxide at Fixed Chlorite Concentrations

5.5 The Effect of $\left[\mathrm{ClO}_{2}^{-}\right]_{0} /[\mathrm{PTU}]_{0}$ ratio on the absorbance traces at $360 \mathrm{~nm} \ldots \ldots \ldots . .130$

5.6a Direct reaction of PTU and Chlorine Dioxide Monitored at $360 \mathrm{~nm}$ with Oxidant to Reductant Ratios below Stoichiometric amount. 133

5.6b Chlorine Dioxide Oxidation at Oxidant to Reductant Ratios; $\left[\mathrm{ClO}_{2}^{-}\right]_{0} /[\mathrm{PTU}]_{0}>$ 2. 135

5.6c Effect of Chlorite on the Oxidation of PTU by Chlorine Dioxide...................... 136

5.7a Depletion of PTU Monitored at $249 \mathrm{~nm}$ for the Oxidation by Chlorite ............. 137

5.7b Relationship between Initial Rate of Consumption of PTU and the Initial Concentrations 138

5.7c Depletion of PTU in its Oxidation by Chlorine Dioxide Monitored at $249 \mathrm{~nm} .139$ 
5.8a Absorbance Traces at $249 \mathrm{~nm}$ Showing the Effect of Acid on the Rate of Consumption of PTU at Low Acid Concentrations ............................................ 140

5.8b Initial Rate Plots for Low Acid Concentrations................................................... 140

5.8c Absorbance Traces at $249 \mathrm{~nm}$ Showing the Effect of Acid on the Rate of Consumption of PTU at High Acid Concentrations .......................................... 141

5.9a Chlorine Dioxide Oxidation. Chlorine Dioxide Variation.................................. 142

5.9b Chlorine Dioxide Oxidation. Effect of Low Acid Concentrations ..................... 143

5.9c Chlorine Dioxide Oxidation. Effect of High Acid Concentrations...................... 143

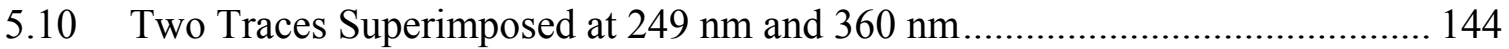

5.11 Simulations of the Data Shown in Figure 7 using Mechanism in Table 5.2 ….. 161

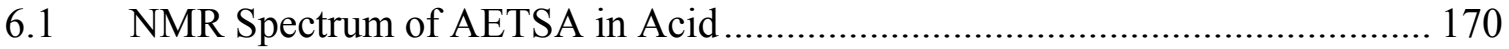

6.2 Effect of Chlorite on the Absorbance Activity at $360 \mathrm{~nm}$................................ 172

6.3 Plot of Induciton Time vs. $1 /\left[\mathrm{ClO}_{2}^{-}\right]_{0}$ using Stoichiometric Excess of Chlorite. 173

6.4 Absorbance Traces at $360 \mathrm{~nm}$ Showing Effect of Acid on the Oxidation of

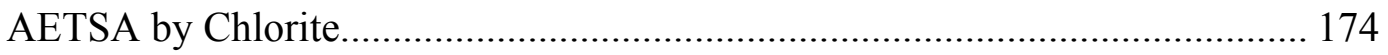

6.5 Induction Time vs. the Inverse of the Initial Acid Concentrations $\left[\mathrm{H}^{+}\right]_{0} \ldots \ldots \ldots . . . .175$

6.6 Effect of Varying [AETSA] $]_{0}$ on Formation of Chlorotaurine at Fixed Chlorite

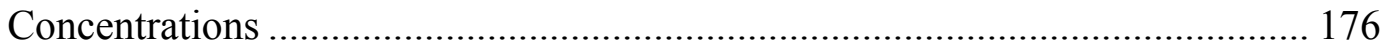

6.7 Absorbance Traces of AETSA Oxidation by $\mathrm{ClO}_{2}$ in acidic solution................ 177

6.8a Absorption Spectrum of AETSA Showing Peak at $241 \mathrm{~nm}$.............................. 181

6.8b Absorbance Traces Showing Consumption of AETSA by Chlorite at Varying $\left[\mathrm{H}^{+}\right]_{0}$ 182 
6.8c Plot of Initial Rate vs. $\left[\mathrm{H}^{+}\right]_{0}$ Showing a Hint of Saturation Kinetics as Acid Concentrations are Increased

6.9 Results of Modeling Reaction Mechanism Shown in Table 6.1

7.1 Typical Phase Plane for an Excitable System............................................... 205

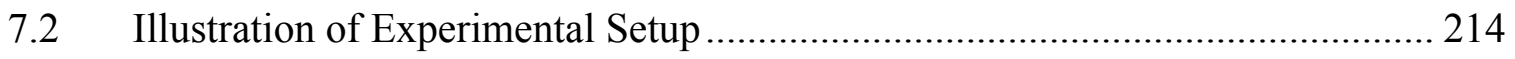

7.3 Image of the Illumination Pattern Projected onto the Gel .............................. 215

7.4 Images of an Oxidation Wave (white) Propagating into an Excitable Light Sensitive BZ Medium .......................................................................... 218

7.5 V-Shaped Curve that Encloses the Region in Forcing Amplitude - Forcing Frequency where the Initiation of Chemical Waves is Possible with Subthreshold Variations in Light Intensity.

7.6 Bifurcation Diagram Showing Firing Number $f n$ as a Function of Forcing Period for $\alpha=0.14$ 220

7.7 Phase diagram showing values of parameters $v / v_{0}$ and $\alpha$ that lead to initiation of waves. 225

7.8 Bifurcation Diagram showing Firing Number $f n$ as a Function of Normalized Forcing Frequency $v / v_{0}$ Illustrating the Dominance of 1:q Mode Locking Regions 226

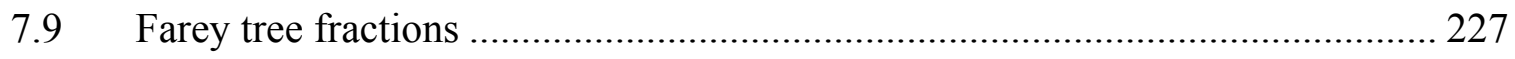

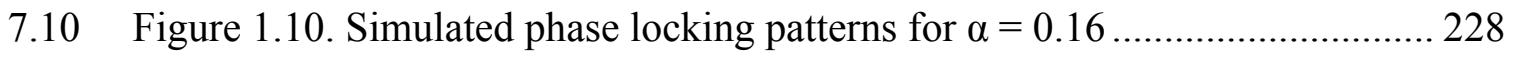

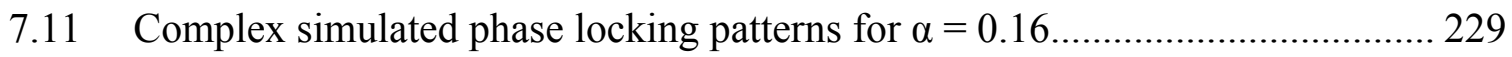

7.12 Images of an oxidation wave (white) propagating in two-dimensional excitable media. 230 
8.1a Calculated Space-Time Plots Showing Profiles of NAD ${ }^{\bullet}$ Radicals................... 260

8.1b Space-time plots showing profiles of NAD ${ }^{\circ}$ radicals demonstrating the wave propagating all the way to the boundary ................................................... 261

8.2 Simulated space-time plots of profiles of NAD ${ }^{\circ}$ radicals ................................. 261 


\section{LIST OF TABLES}

1.1 Sulfur-Oxygen Compounds in Various Oxidation States ................................... 8

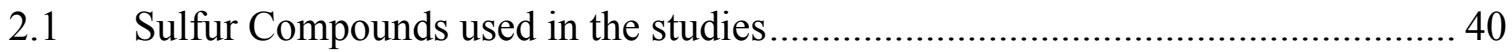

3.1 Chlorine Dioxide/TTTU Reactions in High Acid ........................................ 75

4.1 The Chlorite-Chlorine Dioxide-Trimethylthiourea Reaction Network .............. 111

5.1 Analysis of Absorbance Data from Figure 6b ............................................. 134

5.2 The Chlorite-Chlorine Dioxide-Phenylthiourea Reaction Network ................... 158

6.1 Full Mechanism for the Chlorite-AETSA Reaction ...................................... 189

7.1 The Field, Körös and Noyes (FKN) Mechanism ........................................... 203

7.2 State Variables of Some Representative Excitable Media............................... 207

7.3 Concentrations of Stock Solutions ...................................................................... 210

7.4 Composition of catalyst-free BZ solution................................................. 212

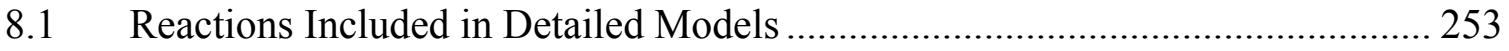




\section{PREFACE}

Nonlinear phenomena occurring in conditions far from equilibrium are of crucial importance in living systems [1]. Studies into nonlinear processes were initially hampered by earlier scientists' beliefs that these processes violated the laws of thermodynamics. The complexity of the reaction mechanisms involved and difficulty in solving nonlinear systems analytically also contributed to the initial reluctance of many scientists to be involved in this field [2]. Coupling of nonlinear chemical processes with diffusion may result in spatiotemporal ordering, leading to the formation of dissipative structures such as chemical waves [3-6]. In this dissertation, we report on the kinetics and mechanisms of nonlinear chemical systems that involve oxyhalogen-sulfur chemistry. We also report on the generation of chemical waves in reaction-diffusion systems of well known chemical oscillators.

This dissertation is composed of two parts, which are linked by the nonlinear character of all of the systems discussed in both parts.

Part I, S-oxygenation of selected thiocarbamides, deals with the chemical kinetics and mechanisms of selected substituted thioureas, which are presented in Chapters 1 to 6 . Chapter 1 is an overall introduction to the field of organosulfur chemistry, with emphasis on the chemical reaction dynamics and the biological significance of thiocarbamides and their bioactivation. A general overview of the instrumentation and experimental techniques used in the investigation of S-oxygenation of thiocarbamides reported in Part I is given in Chapter 2. In Chapter 3, a report on the oxidation of tetramethylthiourea, TTTU, by chlorite in acidic media is presented [7]. Chapter 4 reports on the oxidation of 
trimethylthiourea, TMTU, by chlorite and chlorine dioxide [8]. Oxidation of phenylthiourea, PTU [9], and 2-aminoethanethiolsulfuric acid, AETSA [10], are reported in chapters 5 and 6 , respectively.

Part II, Chemical waves in the Belousov-Zhabotinsky (BZ) and PeroxidaseOxidase $(\mathrm{PO})$ reaction systems, reports on the generation of trigger waves in chemical systems. Chapters 7 describes experimental and numerical investigations into the generation of resonance induced chemical waves in an excitable $\mathrm{BZ}$ reaction-diffusion system. In Chapter 8, a review of previous experimental and theoretical studies of the peroxidase-oxidase reaction is presented. Results of numerical simulations of an oscillatory PO reaction-diffusion system are also presented, showing the generation of trigger waves. 


\section{References}

1. A. Babloyantz, Molecules, Dynamics, and Life: An Introduction to SelfOrganization of Matter, (Wiley, New York, 1986).

2. S. H. Strogatz, Nonlinear Dynamics and Chaos: With Applications to Physics, Biology, Chemistry, and Engineering, (Addison-Wesley Pub, Reading, 1994).

3. A. M. Turing, "Chemical basis of morphogenesis," Trans. Roy. Soc. B237, 37-72 (1952).

4. A. M. Turing, "The chemical basis of morphogenesis," B. Math. Biol. 52, 153-197 (1990).

5. K. Showalter, "Pattern formation in a ferroin-bromate system," J. Chem. Phys. 73, 3735-3742 (1980).

6. M. J. B. Hauser and R. H. Simoyi, "Inhomogeneous precipitation patterns in a chemical wave," Phys. Lett. A 191, 31-38 (1994).

7. T. R. Chigwada and R. H. Simoyi, "S-oxygenation of thiocarbamides III: Oxidation of tetramethylthiourea by chlorite in acidic media," to be submitted.

8. T. R. Chigwada and R. H. Simoyi, "S-Oxygenation of Thiocarbamides II: Oxidation of Trimethylthiourea by Chlorite and Chlorine Dioxide," J. Phys. Chem. A 109, 1094-1104 (2005).

9. T. R. Chigwada, E. Chikwana, and R. H. Simoyi, "S-Oxygenation of Thiocarbamides I: Oxidation of Phenylthiourea by Chlorite in Acidic Media," J. Phys. Chem. A 109, 1081-1093 (2005).

10. C. R. Chinake, C. Mundoma, R. Olojo, T. Chigwada, and R. H. Simoyi, "Oxyhalogen-sulfur chemistry: Kinetics and mechanism of the oxidation of a Bunte salt 2-aminoethanethiolsulfuric acid by chlorite," Phys. Chem. Chem. Phys. 3, 4957-4964 (2001). 


\section{CHAPTER 1}

\section{Introduction}

\subsection{INTRODUCTION}

Sulfur is a pale yellow, tasteless, odorless, non-metallic brittle solid occurring widely in nature as iron pyrites $\left(\mathrm{FeS}_{2}\right)$, galena $(\mathrm{PbS})$, sphalerite $(\mathrm{ZnS}$ or $\mathrm{FeS})$, cinnabar

$(\mathrm{HgS})$, stibnite $\left(\mathrm{Sb}_{2} \mathrm{~S}_{3}\right)$, gypsum $\left(\mathrm{CaSO}_{4} \cdot 2\left(\mathrm{H}_{2} \mathrm{O}\right)\right)$, epsom salts $\left(\mathrm{MgSO}_{4} \cdot 7\left(\mathrm{H}_{2} \mathrm{O}\right)\right)$, colestite $\left(\mathrm{SrSO}_{4}\right)$ and barite $\left(\mathrm{BaSO}_{4}\right)$. Native or free sulfur forms occur in nature in the vicinity of volcanoes and hot springs, and are recovered using the Frasch process [1]. The word sulfur used interchangeably with the word brimstone ('the rock that burns'), may have one or two derivations: the Sanskrit word 'sulvere' or the Latin word 'sulphurium' both meaning sulfur.

Sulfur, the tenth most abundant element in the universe, is an essential element of all living organisms and comprises about $0.25 \%$ of total human body weight, similar to potassium [2,3]. The element sulfur is present in four amino acids: methionine, an essential amino acid; cystine and cysteine, which are synthesized from methionine; and taurine which is synthesized from methionine and cysteine. It is also present in two vitamins; thiamine, which is important to skin; and biotin, which is important to hair; and is necessary for the formation of collagen, keratin, insulin and heparin. Sulfur is important in cellular respiration [4], and as part of proteins where the disulfide bonds function to stabilize the tertiary bonds [2,5]. Sulfur is used in the manufacturing of sulfides, insecticides, plastics, enamels, dyes, bleaching agents, fertilizers, gunpowder, laxatives, fungicides, fumigants, and sulfuric acid [6-8]. Sulfur, so essential to life, and 
found in every cell of every organism, has been all but ignored by not only researchers but also by dietitians and physicians.

Sulfur is also important in environmental chemistry, where sulfur dioxide $\left(\mathrm{SO}_{2}\right)$ and nitrogen oxides $\left(\mathrm{NO}_{\mathrm{x}}\right)$ are the primary causes of acid rain, which causes substantial damage to the natural environment in affected regions. Hydrogen sulfide $\left(\mathrm{H}_{2} \mathrm{~S}\right)$, another important environmental pollutant, if metabolized in high concentrations can lead to death via respiratory paralysis. The occurrence of other sulfur compounds as environmental pollutants has resulted in the increased interest in sulfur chemistry.

Many of the sulfur compounds are either toxic or react to form toxic end products, hence the mechanisms by which such reactions occur needs to be elucidated. Studies over the years have shown that such reactions usually display a wide range of complex behavior. The research into the study of sulfur and its compounds has often been hampered by the existence of nonlinear features such as autocatalysis [9], autoinhibition [10], free radical mechanisms [11], polymerizations [12], variable stoichiometries [13], and a wide range of $\mathrm{pH}$ values over which the reactions can occur [14]. Such nonlinearities heighten the possibility of generating chemical instabilities [15]. The origin of such varied exotic behavior from such simple chemical systems is largely responsible for the unwillingness of many kineticists to study the reaction dynamics of reactions involving organic sulfur compounds. However, it is precisely because of the varied dynamics observed with sulfur chemistry that make sulfur compounds ideal for biological mechanisms. The progress in sulfur research [16] has also been hampered by the historically rigid differentiation between inorganic and organic sulfur chemistry. The sulfur compounds used in this study and that of other researchers are organic, and most of 
the relevant sulfur chemistry in the human body is of organic origin, although the products of their oxidation e.g. sulfates are not.

Most of the knowledge on sulfur compounds has been a byproduct of synthetic, analytical and metabolic investigations in other areas [16]. The range of physiological effects associated with organic sulfur chemistry, span from therapeutic to toxic, with several levels of each extreme represented [17]. Surprisingly, previous studies on the mechanistic details of physiological effects of organosulfur compounds have relied on cause and effect; a very inefficient way of rationalizing the effects of such a wide range of physiologically active compounds. Hence there is a need for systematic studies in which physiological effects are assessed based on the reactivities of the compounds and the mechanistic details elucidated.

One of the most significant parameters associated with the physiological role of organic sulfur compounds is the carbon-sulfur bond. Its cleavage or lack thereof, determines a series of events that are extremely important in ascertaining the physiological effects associated with the specific sulfur compound. Use of organic sulfur compounds as drugs is usually determined by the type and nature of the carbon-sulfur bond(s) present in the parent compound. Aside from carbon-sulfur bond cleavage [18], there are four other main reactions undertaken by organosulfur compounds in physiological environments. These are sulfide-disulfide equilibrium [19], free radical formation and detoxification [20], metabolic activation (enzymatic and non-enzymatic) [21], and metabolic S-oxidations [22]. These processes are responsible for most of the known physiological effects of organosulfur compounds. The objective of this study is partly to elucidate the mechanism of the carbon-sulfur bond cleavage (or lack thereof) of 
selected organosulfur compounds, with the ultimate aim of being able to predict the physiological effects of the parent compound.

\subsubsection{Sulfur - oxygen compounds}

Sulfur chemistry is often complicated by the ability of the sulfur atom to assume a wide range of possible oxidation states which range from -2 to +6 [23], as it is able to expand electronically into the accessible d-orbitals. This makes it possible for sulfur to form a series of oxyanions and other compounds at those oxidation states that are chemically, and biologically active. Such compounds can undergo redox reactions with liberation of energy as the oxidation state of sulfur increases. Electron flow from an electron donor via several intermediate electron carriers towards an electron acceptor is the means by which biochemical energy is generated [24-26]. These carriers are located in an orderly manner at, and within membranes. The transport of electrons across these membranes gives rise to potential differences, which in turn liberates energy for metabolic processes [25]. Thus an organism needs suitable electron donors in addition to electron acceptors. Sulfur compounds in higher states act as sinks for such reactions. Reduced sulfur compounds are excellent donors while intermediates between sulfide and sulfate can play both roles depending on the requirements [17]. There are a vast number of compounds in which one or more oxygen atoms are linked to the sulfur center, some of them are listed in Table 1.1.

Although several oxidation states are possible, there is a special stability associated with oxidation states $-2,0$, and +6 . Stability of these states depends also on the chemical environment. The 0 oxidation state is especially stabilized by polymerization, 
where $\mathrm{S}_{8}$ is the most stable form of $\mathrm{S}(0)$ [27], but is not stable in a sulfenic acid RS-OH [28]. The +2 state on the other hand can be stabilized in a sulfinic acid, but not in $\mathrm{SCl}_{2}$ [29].

Table 1.1: Sulfur-Oxygen Compounds in Various Oxidation States

\begin{tabular}{lllll}
\hline Oxidation & Compound & Structure & Compound & Structure \\
\hline-2 & Thiols & R-SH & Thioethers & R-S-R \\
-1 & Disulfides & R-S-S-R & Sulfoxides & R-S(O)R \\
0 & Sulfenic Acids & R-S-OH & Sulfones & R-S $(\mathrm{O})_{2} \mathrm{R}$ \\
+2 & Sulfinic Acids & R-S $(\mathrm{O}) \mathrm{OH}$ & Sulfamates & R-NH-S $(\mathrm{O})_{2} \mathrm{OH}$ \\
+4 & Sulfonic Acids & R-S $(\mathrm{O})_{2} \mathrm{OH}$ & & \\
+6 & Sulfate Esters & R-O-S $(\mathrm{O})_{2} \mathrm{OH}$ & & \\
\hline
\end{tabular}

Adapted from "Sulfur-Oxygen Compounds" by A.G. Renwick [30]

The chemical and biological properties of organic sulfur-oxygen compounds depend on the nature of the substituents on the sulfur, carbon and oxygen atoms. The carbon sulfur bond will only be strongly influenced by the substituents on the carbon and sulfur atoms [31]. This chapter concentrates on the main classes of sulfur-oxygen and their metabolic precursors that are relevant to the work presented in the following chapters of this dissertation. Emphasis will be placed on aromatic and methyl substituted and non-substituted thiocarbamides and their derivatives in order to best understand reactivities, which can be affected by conjugation of the reactive thiocarbonyl group. 


\subsubsection{Metabolic Activation of S-atom via S-oxygenation}

The sulfur-oxygen bond is one of the most important parameter in determining physiological activity of organic sulfur compounds. The only way to study the carbonsulfur bond is through S-oxygenations.

Sulfur compounds undergo a variety of metabolic reactions, namely oxidations, reductions, hydrolysis, and conjugations [32]. Sulfur in thiocarbamides, and most organosulfur compounds is nucleophilic, implying that such compounds are susceptible (in physiological environments) to metabolic oxidations [33]. The oxidation of these compounds should thus represent an important aspect of sulfur metabolism. These oxidations appear to be involved in many cellular functions, including the reductive degradation of polypeptide hormones and proteins, regulation of protein synthesis, maintenance of intracellular redox potential, and protection of cells from oxidative damage [34], to name just a few.

Adverse toxicological effects of organosulfur compounds such as pulmonary edema [35-37], thyroid depression [38-41], and liver necrosis [41-45] are widely believed to be due to the formation of S-oxides. S-oxygenation of the thionocarbonyl or thiourea moiety $(>\mathrm{C}=\mathrm{S})$ is considered to be the first step towards the bioactivation of organosulfur compounds. This reaction is catalyzed primarily by the flavin containing monooxygenases (FMO) also known as FAD-containing monooxygenase [46], and the microsomal cytochrome P450 system of enzymes (P450). Facile oxidations of the organosulfur compounds by reactive oxygen species, e.g. hydrogen peroxide, and superoxide ion, however, often complicate the interpretation of in vitro studies [33]. 
There are two possible ways of oxidizing the thiourea moiety of the organosulfur compounds: a) through electrophilic attack, and b) through successive addition of oxygen to the sulfur center [47]. Three possible oxyacids can arise from the stepwise oxidation of the sulfur center, namely: i) sulfenic acids (S-oxide, $\mathrm{RSOH}$ ), ii) sulfinic acids (S-dioxide, $\mathrm{RSO}_{2} \mathrm{H}$ ), and iii) sulfonic acids (S-trioxide, $\mathrm{RSO}_{3} \mathrm{H}$ ) [48]. These S-oxides are the reactive intermediates whose reactivity might lead to toxicity.

\subsubsection{Enzymes involved in S-oxygenation}

The first step in the bioactivation of thiono compounds, S-oxygenation, is believed to be catalyzed mostly by the membrane bound flavin-containing monooxygenase, to yield the sulfenic acid which is highly unstable [49]. FMOs, are proteins whose apparent molecular weight is in the range of 52,000 to 64,000 Daltons. The FMOs contain a single molecule of flavin adenine dinucleotide (FAD) as the active group [50,51].

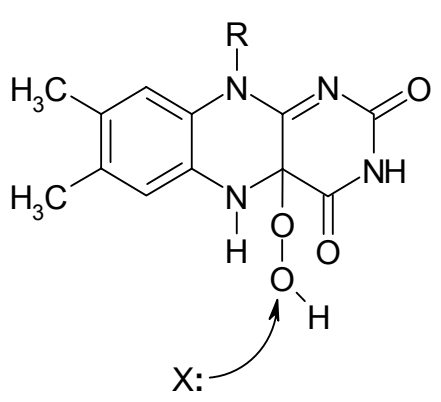

1

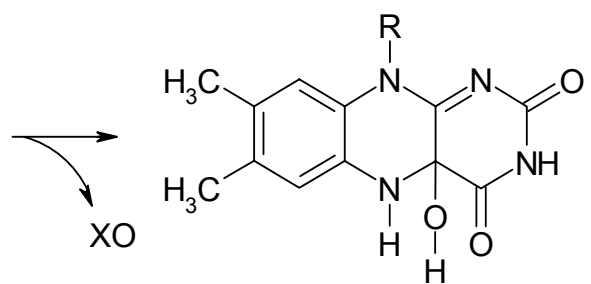

2

Figure 1.1: Active site of Flavin-Containing Monooxygenase (FMO) [52] 
The FADH-OOH complex, shown in Figure 1.1, is the $4 \alpha$-hydroxyperoxyflavin, a stable intermediate in the absence of the substrate [52]. This stability, however, is only relative since the release of superoxide and hydrogen peroxide is documented [53]. In the presence of a nucleophilic substrate (:X), e.g. sulfur or nitrogen, attack occurs on the distal oxygen atom to yield the oxygenated product $\mathrm{XO}$ with a water molecule playing a critical role in the transition state [52-56].

Cytochrome P450 enzymes also catalyze some of these oxidation reactions, e.g. in the bioactivation of methimazole [57], and $\alpha$-naphthylthiourea (ANTU) [46,58]. Cytochrome P450's are known to catalyze many if not all reactions catalyzed by FMO's. Cytochrome P450, like FMO also comprises of the FAD molecule $[59,60]$. The P450 enzymes are electron carriers that contain an iron porphyrin ring as the prosthetic group. The protein bound iron of the porphyrin ring facilitates the transfer of electrons by changing oxidation states between the ferrous $\left(\mathrm{Fe}^{2+}\right)$ and ferric $\left(\mathrm{Fe}^{3+}\right)$ states [59]. These enzymes (cytochrome P450) are utilized in other reactions aside from bioactivation of sulfur compounds, including degradation of xenobiotics, generation of hormones and biosynthesis of a variety of important biological compounds [61]. The cytochrome P450 family is a major participant in nearly all metabolisms of pharmaceutical reagents.

\subsubsection{Thiocarbamides $\left(\mathbf{R}^{1} \mathbf{R}^{2} \mathrm{NC}(=\mathbf{S}) \mathrm{NHR}^{3} \leftrightarrow \mathbf{R}^{1} \mathbf{R}^{2} \mathrm{C}(\mathrm{SH})=\mathrm{NR}^{3}\right)$}

Thiocarbamides are a family of compounds of thioamides with thiourea as the simplest compound in this class. The thionocabonyl moiety of thiocarbamide is known to be responsible for the wide use of thiocarbamides as insecticides [62,57], as therapeutic agents [63], as goitrogens [64], as non-nucleoside inhibitors of HIV-I reverse 
transcriptase $[65,66]$, to name a few. Thiocarbamides can be grouped, broadly, into four biologically active groups: rodenticides ( $\alpha$-napthylthiourea) [67-69], antithyroids for treating hyperthyroidism (propylthiouracil) [41,70,71], barbiturates (thiopentone) [72], and radical scavengers (dimethylthiourea) [73-75]. Thiourea, TU, the simplest thiocarbamide is an important industrial chemical, and is used in rubber production as a vulcanization accelerator [76-78], and as a component of solutions for ore leaching [79], for example. Thiourea is a strong goitrogen and its antithyroid activity is believed to lead to a disruption of the pituitary-thyroid hormonal regulatory system [64]. TU is also thought to be a potential melanoma-targeting agent [80].

The only naturally occurring thiocarbamide is ergothioneine found in large quantites in mammalian blood cells [81]. In contrast, the synthetic thiocarbamides have a wide and extensive usage, from intermediates in organic reactions to therapeutic agents. Methimazole, for example, marketed under the trade name Tapazole is used for medical treatment of hyperthyroidism due to Graves' disease [63,82]. Unfortunately, methimazole's toxicity sometimes makes it unsuitable as a drug and is substituted by the less effective propylthiouracil $[83,84]$. Phenylthiocarbamide or phenylthiourea (PTU) [85], and thiouracil [86] were also used to treat hyperthyroidism, but their uses were discontinued due to their toxic side effects . $\alpha$-naphthylthiourea [69,87] and N-methyl-N'(hydroxymethyl)thiourea [88] are used as rodenticides and bactericides respectively. The thiocarbamide diafenthurion, is used as an insecticide [62].

Dimethylthiourea, DMTU, is one of the most efficient scavengers of reactive, toxic oxygen species in vitro, and reduces oxidative injury in many biological systems [74,89-91]. DMTU is one of the most efficient scavengers known for hydrogen peroxide, 
hydroxyl radical, and hypochlorous acid [73,92]. DMTU is used in the textile industry as an antioxidant [93]. Diphenylthiourea, DPTU, and dibutylthiourea (DBTU), are also known to have antioxidant properties [94].

Thiocarbamides have been used in the development of promising potent HIV-1 non-nucleoside reverse transcriptase inhibitors (NNRTI) [66,95]. The range of physiological effects derived from a single thiourea compound makes these compounds well suited as dual function microbicidal contraceptives potentially capable of preventing HIV transmission as well as providing fertility control [95]. Compounds such as (N-[2(2-chlorophenethyl)]-N'-[2-(5-bromopyridyl)]-thiourea) and (N-[2-(5-bromopyridil)]-N'[2-(2-(1-cyclohexenyl)ethyl]-thiourea) were found to possess potent anti-HIV activity as well as sperm-immobilizing activity [66]. The spermicidal activity of these drugs makes them to be efficient replacements of current commercially available spermicidal microbicides such as the detergent-based nonoxynol-9, which disrupt cell membranes [66]. The first HIV experimental drug to be tested was Trovirdine [65,57].

\subsubsection{Toxicity of Thiocarbamides}

The thionocarbonyl or thiourea moiety $(>\mathrm{C}=\mathrm{S})$ of thiocarbamides asides for it being a pharmacophore, is also a potential toxophore. Toxicological effects of thiocarbamides include thyroid depression, pulmonary edema, and liver necrosis. Thus, a large number of potentially useful drugs containing the thionocarbonyl moiety are often limited in their use due to the toxic side effects associated with the compounds. The associated toxicity of thiocarbamides has been linked to their oxidative metabolism, especially S-oxygenation. 
It was reported in a 1961 publication that phenylthiourea is extremely toxic to rats, but that diphenylthiourea is not [96]. There have been several theories postulated on the varying degrees of toxicities of thioureas. One possibility was the nature of the metabolic end-products of the thioureas. In some cases, thioureas are desulfurized to their corresponding ureas e.g. phenylthiourea and thiourea. N,N-diphenylthiourea which was found to be non-toxic, is excreted primarily as the ring-metabolized products with the thione group intact, while phenylthiourea was excreted as sulfate and phenylurea. Thus, the toxicity of the thiocarbamide is determined by its metabolic pathway, intermediates and/or products. The toxicity of the thiocarbamides is related to the extent of desulfurization, with the more highly desulfurized compound being the most toxic. Hepatic toxicity is almost inevitable with most drugs that contain the carbon-sulfur bond [97]. There have been reports of fulminant hepatitis, hepatic necrosis, encephalopathy, and even death in some cases.

Subtle differences in substituted thioureas can impart vastly different degrees of toxicity. For example, while $\alpha$-naphthylthiourea produces pulmonary edema and pleural effusion in rats, its oxidation gives $\alpha$-naphthylurea, an innocuous substance to rats [98]. The degree of toxicity of thiocarbamides is also species-dependent $[99,100]$. Ethylenethiourea, for example, is known to be toxic in rats and mice, with its toxicities including goitrogenicity, carcinogenicity, and with teratogenic effects [101-103], although cats and rabbits [104], however, are not susceptible to its teratogenic effects [105]. These subtle differences may be related to the fact that the major pathways of metabolism in rats are oxidative whereas in cats, S-methylation predominates [106,107]. 
In order to understand why similar thiocarbamides possess varying toxicity effects, which in some cases are species dependent, and also understand the origin of nonlinearities observed with sulfur compounds, a series of studies were started in our laboratory [48], with the aim of systematically investigating and hopefully deducing a generalized algorithm for the oxidation of the sulfur center. Oxidation of the sulfur center generally displays nonlinear and exotic dynamic behavior which includes clock reaction mechanisms [108,109], chemical oscillations [15,110], oligooscillations [111], chemical chaos [112,113], $\mathrm{pH}$ oscillations [14,114], spatial patterns [115-117], and traveling waves [118]. Knowledge of the oxidation mechanisms of the sulfur center is essential in the elucidation of these observed nonlinear dynamics. This series of studies has so far resulted in the derivation of a simple algorithm which involves successive addition of oxygen onto the sulfur center up to the sulfonic acid stage, via non-radical two-electron equivalents transfer processes yielding a sulfate as the final product due to cleavage of the carbon-sulfur bond $[29,119]$. The rate-determining step was established to be the initial oxidation of the organosulfur compound, to the unstable sulfenic acid. The studies have also revealed, however, that nearly every organosulfur compound presents a unique reactivity, and has to be studied individually. This feature of diversity in oxidation mechanisms of closely related sulfur compounds has also been observed in biological chemistry [120]. Hydroxymethanesulfonic acid, for example, can be easily oxidized to a carboxylic acid and sulfate [121], while its homologue, the biologically important isethionic acid is extremely inert and is only oxidized only by the most powerful oxidizing agents [122]. This dissertation will involve a continuation of the studies aimed 
at systematically elucidating the S-oxygenation mechanisms of substituted thioureas in different electronic environments. 


\section{References}

1. D. Cavallini, G. E. Gaull, and V. Zappia, Natural Sulfur Compounds: Novel Biochemical and Structural Aspects, (Plenum Press, New York, 1980).

2. N. A. Campbell, Biology: Concepts \& Connections, (Benjamin Cummings, San Francisco, 2003).

3. C. Meyer, Sulfur, Energy, and Environment, (Elsevier Scientific Pub. Co., New York, 1977).

4. J. W. Hole, Human Anatomy and Physiology, (W.C. Brown Co., Iowa, 1981).

5. C. C. Price and S. Oae, Sulfur Bonding, (Ronald Press Co, New York, 1962).

6. G. Rayner-Canham and T. Overton, Descriptive Inorganic Chemistry, (W.H. Freeman, New York, 2003).

7. U. H. F. Sander, Sulfur, Sulfur Dioxide, and Sulfuric Acid: An Introduction to Their Industrial Chemistry and Technology, (British Sulphur Corp., London, 1984).

8. W. N. Tuller, The Sulphur Data Book, (New York, McGraw-Hill, 1954).

9. R. H. Simoyi and I. R. Epstein, "Systematic design of chemical oscillators. 40. Oxidation of thiourea by aqueous bromine: autocatalysis by bromide," J. Phys. Chem. 91, 5124-5128 (1987).

10. R. H. Simoyi, I. R. Epstein, and K. Kustin, "Systematic design of chemical oscillators. 49. Kinetics and mechanism of the autoinhibitory iodine-thiocyanate reaction," J. Phys. Chem. 93, 2792-2795 (1989).

11. Y. Luo, M. Orban, K. Kustin, and I. R. Epstein, "Mechanistic study of oscillations and bistability in the copper(II)-catalyzed reaction between hydrogen peroxide and potassium thiocyanate," J. Am. Chem. Soc. 111, 4541-4548 (1989).

12. D. Barnard, "Spontaneous decomposition of aryl thiolsulfinates," J. Chem. Soc. Abstracts, 4675-4676 (1957).

13. R. H. Simoyi, "New bromate oscillator: the bromate-thiourea reaction in a CSTR," J. Phys. Chem. 90, 2802-2804 (1986).

14. G. Rabai, M. Orban, and I. R. Epstein, "Systematic design of chemical oscillators. 64. Design of pH-regulated oscillators," Acc. Chem. Res. 23, 258-263 (1990).

15. M. Alamgir and I. R. Epstein, "Systematic design of chemical oscillators. Part 31. New chlorite oscillators: Chlorite-bromide and chlorite-thiocyanate in a CSTR," J. Phys. Chem. 89, 3611-3614 (1985). 
16. A. Senning, Sulfur in Organic and Inorganic Chemistry, (M. Dekker, New York, 1971).

17. R. J. Huxtable and W. M. Lafranconi, Biochemistry of Sulfur, (Plenum Press, New York, 1986).

18. V. Lepentsiotis, R. van Eldik, D. M. Stulov, and S. V. Makarov, "Investigation of the redox interaction between iron(III) 5,10,15,20-tetrakis(p-sulfonatophenyl)porphyrinate and aminoiminomethanesulfinic acid in aqueous solution," J. Chem. Soc. Dalton 2915-2920 (1998).

19. A. V. Zavialov, M. Gaestel, T. Korpela, and V. P. Zav'yalov, "Thiol-disulfide exchange between small heat shock protein 25 and glutathione," Biochim Biophys. Acta 1388, 123-132 (1998).

20. Y. Minamiyama, S. Takemura, K. Koyama, H. Yu, M. Miyamoto, and M. Inoue, "Dynamic aspects of glutathione and nitric oxide metabolism in endotoxemic rats," Am. J. Physiol. 271, G575-G581 (1996).

21. T. Mizutani, K. Yoshida, M. Murakami, M. Shirai, and S. Kawazoe, "Evidence for the involvement of n-methylthiourea, a ring cleavage metabolite, in the hepatotoxicity of methimazole in glutathione-depleted mice: Structure-toxicity and metabolic studies," Chem. Res. Toxicol. 13, 170-176 (2000).

22. L. L. Poulsen, R. M. Hyslop, and D. M. Ziegler, "S-Oxygenation of N-substituted thioureas catalyzed by the pig liver microsomal FAD-containing monooxygenase," Arch. Biochem. Biophys. 198, 78-88 (1979).

23. S. C. Mitchell and R. M. Nickson, "Metabolism of sulfur-containing xenobiotics," Sulfur Reports 13, 161-195 (1993).

24. A. A. Krasnovskii, "Evolution of uphill electron transfer in photosynthesis," in Science and Scientists: Essays by Biochemists, Biologists and Chemists, edited by M.Kageyama, K.Nakamura, and T.Oshima, (Japan Scientific Societies Press, Tokyo, 1981) p. 47-55.

25. A. A. Krasnovsky, "Evolution of uphill electron transfer," Biosystems 14, 81-87 (1981).

26. V. A. Shuvalov and A. A. Krasnovskii, "Photochemical electron transfer in reaction centers of photosynthesis," Biofizika 26, 544-556 (1981).

27. F. A. Cotton, Advanced Inorganic Chemistry, (Wiley, New York, 1999).

28. B. Testa, "Monooxygenase-catalysed oxidation of oxygen and sulfur containing compounds," in The Metabolism of Drugs and Other Xenobiotics. Biochemistry of Redox Reactions, edited by B.Testa, (Academic Press, London, 1995) p. 235-275. 
29. C. R. Chinake, R. H. Simoyi, and S. B. Jonnalagadda, "Oxyhalogen-sulfur chemistry: The bromate-(aminoimino)methanesulfinic acid reaction in acidic medium," J. Phys. Chem. 98, 545-550 (1994).

30. A. G. Renwick, "sulfur - Oxygen Compounds," in Biological Interactions of Sulfur Compounds, edited by S.Mitchell and C.Steve, (Taylor \& Francis, London, 1996) p. 42-76.

31. W. D. Jones, R. M. Chin, T. W. Crane, and D. M. Baruch, "Carbon-sulfur bond cleavage in thiophene by group 6 metallocenes," Organometallics 13, 4448-4452 (1994).

32. S. B. Park, W. N. Howald, and J. R. Cashman, "S-Oxidative cleavage of farnesylcysteine and farnesylcysteine methyl ester by the flavin-containing monooxygenase," Chem. Res. Toxicol. 7, 191-198 (1994).

33. A. Del Corso, M. Cappiello, and U. Mura, "Thiol dependent oxidation of enzymes: The last chance against oxidative stress," I. J. Biochem. 26, 745-750 (1994).

34. D. T. Organisciak, R. M. Darrow, Y. Jiang, I, G. E. Marak, and J. C. Blanks, "Protection by dimethylthiourea against retinal light damage in rats," Invest. Ophthalmol. Vis. Sci. 33, 1599-1609 (1992).

35. J. B. Mackenzie and C. G. Mackenzie, "Production of pulmonary edema by thiourea in the rat, and its relation to age," Proc. Soc. Exp. Bio. Med. 54, 34-37 (1943).

36. S. N. Giri, M. A. Hollinger, C. E. Cross, and D. L. Dungworth, "Effects of thiourea on pulmonary edema, pleural, and peritoneal effusions and toxicity in rats pretreated with actinomycin D," Toxicology 2, 211-222 (1974).

37. L. S. Gibbs and G. J. Traiger, "Effect of reserpine on N-methylthiobenzamideinduced pulmonary edema: role of lung norepinephrine and hypothermia," Toxicology 54, 311-321 (1989).

38. C. G. Mackenzie and J. B. Mackenzie, "Effect of sulfonamides and thioureas on the thyroid gland and basal metabolism," Endocrinology 32, 185-209 (1943).

39. U. Kabadi and R. Cech, "Therapeutic 131I dose in hyperthyroidism: Role of pretreatment with thionamide," Thyroidology 6, 87-92 (1994).

40. U. Kabadi, "Optimal LT4 dosage in primary hypothyroidism," J. Iowa Med. Soc. 84, 535-537 (1994).

41. R. Bruck, D. Frenkel, H. Shirin, H. Aeed, Z. Matas, M. Papa, L. Zaidel, Y. Avni, R. Oren, and Z. Halpern, "Hypothyroidism protects rat liver from acetaminophen hepatotoxicity," Dig. Dis. Sci. 44, 1228-1235 (1999). 
42. G. Malvaldi, "Hepatic tumors induced by thiobenzamide in the rat," Boll. Soc. Ital. Biol. Sper. 54, 1027-1030 (1978).

43. E. Chieli, G. Malvaldi, and R. Tongiani, "Early biochemical liver changes following thiobenzamide poisoning," Toxicology 13, 101-114 (1979).

44. E. Chieli, G. Malvaldi, and D. Segnini, "The hepatotoxicity of thiobenzamide-Soxide," Toxicol. Lett. 7, 175-180 (1980).

45. E. Chieli and G. Malvaldi, "Role of the microsomal FAD-containing monooxygenase in the liver toxicity of thioacetamide S-oxide," Toxicology 31, 41-52 (1984).

46. A. E. Miller, J. J. Bischoff, and K. Pae, "Chemistry of aminoiminomethanesulfinic and sulfonic acids related to the toxicity of thioureas," Chem. Res. Toxicol. 1, 169-174 (1988).

47. I. Orszagh, G. Bazsa, and M. T. Beck, "Spectrophotometric study of the reversible iodine-thiocyanate interaction," Inorg. Chim. Acta 6, 271-274 (1972).

48. E. Mambo and R. H. Simoyi, "Kinetics and mechanism of the complex oxidation of aminoiminomethanesulfinic acid by iodate in acidic medium," J. Phys. Chem. 97, 13662-13667 (1993).

49. C. J. Decker, D. R. Doerge, and J. R. Cashman, "Metabolism of benzimidazoline2-thiones by rat hepatic microsomes and hog liver flavin-containing monooxygenase," Chem. Res. Toxicol. 5, 726-733 (1992).

50. J. Ozols, "Isolation and Structure of a Third Form of Liver Microsomal Flavin Monooxygenase," Biochemistry 33, 3751-3757 (1994).

51. S. B. Park, J. D. Osterloh, S. Vamvakas, M. Hashmi, M. W. Anders, and J. R. Cashman, "Flavin-containing monooxygenase-dependent stereoselective Soxygenation and cytotoxicity of cysteine S-conjugates and mercapturates," Chem. Res. Toxicol. 5, 193-201 (1992).

52. K. C. Jones and D. P. Ballou, "Reactions of the 4a-hydroperoxide of liver microsomal flavin-containing monooxygenase with nucleophilic and electrophilic substrates," J. Biol. Chem. 261, 2553-2559 (1986).

53. S. E. Patton, G. M. Rosen, and E. J. Rauckman, "Superoxide production by purified hamster hepatic nuclei," Mol. Pharmacol. 18, 588-593 (1980).

54. R. D. Bach, J. J. W. McDouall, A. L. Owensby, and H. B. Schlegel, "Theoretical study of oxygen atom transfer. The role of electron correlation," J. Am. Chem. Soc. 112, 7065-7067 (1990). 
55. R. D. Bach, J. J. W. McDouall, A. L. Owensby, and H. B. Schlegel, "Potential for water catalysis in flavin-mediated hydroxylation. A theoretical study," J. Am. Chem. Soc. 112, 7064-7065 (1990).

56. S. Murahashi, T. Oda, and Y. Masui, "Flavin-catalyzed oxidation of amines and sulfur compounds with hydrogen peroxide," J. Am. Chem. Soc. 111, 5002-5003 (1989).

57. R. C. A. Onderwater, J. N. M. Commandeur, E. J. Groot, A. Sitters, W. M. P. B. Menge, and N. P. E. Vermeulen, "Cytotoxicity of a series of mono- and disubstituted thiourea in freshly isolated rat hepatocytes: A preliminary structuretoxicity relationship study," Toxicology 125, 117-129 (1998).

58. H. A. S. Van den Brenk, H. Kelly, and M. G. Stone, "Innate and drug-induced resistance to acute lung damage caused in rats by a-naphthyl thiourea (ANTU) and related compounds," Brit.J. Exp. Pathol. 57, 621-636 (1976).

59. S. D. Black and M. J. Coon, "P-450 cytochromes: structure and function," Adv. Enzymol. Rel. Areas Mol. Biol. 60, 35-87 (1987).

60. S. D. Black, "On the domain structure of cytochrome P450 102 (BM-3): isolation and properties of a 45-kDa FAD/NADP domain," Biochem. Biophys. Res. Commun. 203, 162-168 (1994).

61. H. V. Vadi and R. A. Neal, "Microsomal activation of thioacetamide-S-oxide to a metabolite(s) that covalently binds to calf thymus DNA and other polynucleotides," Chem. Biol. Interact. 35, 25-38 (1981).

62. I. Ishaaya, Z. Mendelson, and A. R. Horowitz, "Toxicity and growth-suppression exerted by diafenthiuron in the sweetpotato whitefly, Bemisia tabaci," Phytoparasitica 21, 199-204 (1993).

63. A. Shiroozu, K. Okamura, H. Ikenoue, K. Sato, T. Nakashima, M. Yoshinari, M. Fujishima, and T. Yoshizumi, "Treatment of hyperthyroidism with a small single daily dose of methimazole," J. Clin. Endocrinol. Met. 63, 125-128 (1986).

64. L. Jirousek and M. Soodak, "Positive iodine compounds as models of the thyroidal active iodine. Reaction of $\mathrm{N}$-iodosuccinimide and of $\mathrm{N}$-iodophthalimide with thiocarbamide goitrogens," J. Pharmacol. Exp. Ther. 191, 341-348 (1974).

65. R. C. A. Onderwater, J. N. M. Commandeur, and N. P. E. Vermeulen, "Comparative cytotoxicity of N-substituted N'-(4-imidazole-ethyl)thiourea in precision-cut rat liver slices," Toxicology 197, 81-91 (2004).

66. O. J. D'Cruz, T. K. Venkatachalam, and F. M. Uckun, "Novel thiourea compounds as dual-function microbicides," Biol. Reprod. 63, 196-205 (2000). 
67. I. Celik and H. Ozbek, "Effects of subchronic exposure to thiocarbamide on haematological and biochemical constituents of rats," Fresen. Environ. Bull. 13, 159-163 (2004).

68. C. P. Richter, "Substituted thioureas as rodenticides," patent number 2390848 (1946).

69. C. P. Richter, "Biological factors involved in poisoning rats with anaphthylthiourea (ANTU)," Proc. Soc. Exp. Biol. Med. 63, 364-372 (1946).

70. H. Orrego, J. E. Blake, L. M. Blendis, K. Compton, V, and Y. Israel, "Long-term treatment of alcoholic liver disease with propylthiouracil," N. Engl. J. Med. 317, 1421-1427 (1987).

71. M. B. Genter, "Evaluation of olfactory and auditory system effects of the antihyperthyroid drug carbimazole in the Long-Evans rat," J. Biochem. Mol. Toxicol. 12, 305-314 (1998).

72. S. D. Black, M. H. Linger, L. C. Freck, S. Kazemi, and J. A. Galbraith, "Affinity isolation and characterization of cytochrome P450 102 (BM-3) from barbiturateinduced Bacillus megaterium," Arch. Biochem. Biophys. 310, 126-133 (1994).

73. R. B. Fox, "Prevention of granulocyte-mediated oxidant lung injury in rats by a hydroxyl radical scavenger, dimethylthiourea," J. Clin. Invest. 74, 1456-1464 (1984).

74. K. M. Toth, J. M. Harlan, C. J. Beehler, E. M. Berger, N. B. Parker, S. L. Linas, and J. E. Repine, "Dimethylthiourea prevents hydrogen peroxide and neutrophil mediated damage to lung endothelial cells in vitro and disappears in the process," Free Radic. Biol. Med. 6, 457-466 (1989).

75. R. Bruck, H. Aeed, H. Shirin, Z. Matas, L. Zaidel, Y. Avni, and Z. Halpern, "The hydroxyl radical scavengers dimethylsulfoxide and dimethylthiourea protect rats against thioacetamide-induced fulminant hepatic failure," J. Hepatol. 31, 27-38 (1999).

76. A. P. Susamma and A. P. Kuriakose, "Studies on a novel accelerator system in the vulcanisation of polychloroprene rubber," Prog. Rubber Plast. Recycl. Technol. 19, 231-250 (2003).

77. M. Kurien, N. M. Claramma, and A. P. Kuriakose, "A new secondary accelerator for the sulfur vulcanization of natural rubber latex and its effect on the rheological properties," J. Appl. Polym. Sci. 93, 2781-2789 (2004).

78. M. Kurien, A. P. Susamma, and A. P. Kuriakose, "Amidino thiourea as a secondary accelerator in the sulphur vulcanization of natural rubber containing fillers," Prog. Rubber Plast. Recycl. Technol. 20, 133-162 (2004). 
79. S. Oae and T. Okuyama, Organic Sulfur Chemistry: Biochemical Aspects, (CRC Press, Boca Raton, Fla., 1992).

80. K. Ziegler-Skylakakis, S. Nill, J. F. Pan, and U. Andrae, "S-oxygenation of thiourea results in the formation of genotoxic products," Environ. Mol. Mutagen. 31, 362-373 (1998).

81. E. C. Stowell, "Ergothioneine," in Organic Sulfur Compounds, edited by N.Kharasch, (Pergamon Press, New York, 1961) p. 462-490.

82. M. Yoshinari, K. Inoue, T. Nakashima, K. Okamura, A. Shiroozu, H. Nishitani, T. Omae, M. Torisu, and S. Noguchi, "Acid protease activity in thyroid gland from patients with Graves' disease," Metabolism 32, 348-354 (1983).

83. M. S. Balkin, M. Buchholtz, J. Ortiz, and A. J. Green, "Propylthiouracil (PTU)induced agranulocytosis treated with recombinant human granulocyte colonystimulating factor (G-CSF)," Thyroid 3, 305-309 (1993).

84. A. Shiroozu, A. Taurog, H. Engler, and M. L. Dorris, "Mechanism of action of thioureylene antithyroid drugs in the rat: possible inactivation of thyroid peroxidase by propylthiouracil," Endocrinology 113, 362-370 (1983).

85. F. Facchini, A. Abbati, and S. Campagnoni, "Possible relations between sensitivity to phenylthiocarbamide and goiter," Hum. Biol. 62, 545-552 (1990).

86. R. Bayliss, I, "The management of thyroid diseases," Trans. Med. Soc. Lond. 88, 34-39 (1972).

87. S. H. Dieke and C. P. Richter, "Age and species variation in the acute toxicity of anaphthylthiourea," Proc. Soc. Exp. Biol. Med. 62, 22-25 (1946).

88. R. G. Pickard, "Treatment of peritonitis with per- and postoperative irrigation of the peritoneal cavity with noxythiolin solution," Brit. J. Surg. 59, 642-648 (1972).

89. C. J. Beehler, M. L. Simchuk, J. M. McCord, and J. E. Repine, "Effects of dimethylthiourea in hyperoxic injury," Journal of Laboratory and Clinical Medicine 119, 508-513 (1992).

90. C. J. Beehler, M. E. Ely, K. S. Rutledge, M. L. Simchuk, O. K. Reiss, P. F. Shanley, and J. E. Repine, "Toxic effects of dimethylthiourea in rats," J. Lab. Clin. Med. 123, 73-80 (1994).

91. C. J. Beehler, M. L. Simchuk, K. M. Toth, S. K. Drake, N. B. Parker, C. W. White, E. M. Berger, R. J. Sanderson, and J. E. Repine, "Blood sulfhydryl level increases during hyperoxia: A marker of oxidant lung injury," J. Appl. Physiol. 67, 1070-1075 (1989). 
92. C. P. L. Lewis, D. Dinsdale, and B. Nemery, "Potentiation of oxidant-induced toxicity in hamster lung slices by dimethylthiourea," Free Radic. Biol. Med. 16, 561-569 (1994).

93. A. Dooms-Goossens, B. Boyden, A. Ceuterick, and H. Degreef, "Dimethylthiourea, an unexpected hazard for textile workers," Contact Dermatitis 5, 367-370 (1979).

94. A. M. Saillenfait, J. P. Sabate, I. Langonne, and J. De Ceaurriz, "Difference in the developmental toxicity of ethylenethiourea and three N,N'-substituted thiourea derivatives in rats," Fundam. Appl. Toxicol. 17, 399-408 (1991).

95. O. J. D'Cruz, T. K. Venkatachalam, C. Mao, S. Qazi, and F. M. Uckun, "Structural requirements for potent anti-human immunodeficiency virus (HIV) and sperm-immobilizing activities of cyclohexenyl thiourea and urea nonnucleoside inhibitors of HIV-1 reverse transcriptase," Biol. Reprod. 67, 19591974 (2002).

96. R. L. Smith and R. T. Williams, "The metabolism of arylthioureas. I. The metabolism of 1,3-diphenyl-2-thiourea (thiocarbanilide) and its derivatives," J. Med. Pharm. Chem. 4, 97-107 (1961).

97. J. R. Cashman, K. K. Parikh, G. J. Traiger, and R. P. Hanzlik, "Relative hepatotoxicity of ortho and meta monosubstituted thiobenzamides in the rat," Chem. Biol. Interact. 45, 341-347 (1983).

98. P. W. Lee, T. Arnau, and R. A. Neal, "Metabolism of a-naphthylthiourea by rat liver and rat lung microsomes," Toxicol. Appl. Pharmacol. 53, 164-173 (1980).

99. R. A. Neal, T. Kamataki, A. L. Hunter, and G. Catignani, "Monooxygenase Catalysed Activation of Thiono-Sulfur Containing Compounds to Reactive Intermediates," in Microsomes and Drug Oxidations, edited by V.Ullrich, I.Roots, A.Hildebrandt, and R.W.Estabrook, (Pergamon Press, Oxford, 1977).

100. R. A. Neal and J. Halpert, "Toxicology of thiono-sulfur compounds," Annu. Rev. Pharmacol. Toxicol. 22, 321-339 (1982).

101. S. L. Graham, W. H. Hansen, K. J. Davis, and C. H. Perry, "Effects of one-year administration of ethylenethiourea upon the thyroid of the rat," J. Agricult. Food Chem. 21, 324-329 (1973).

102. K. S. Khera and F. Iverson, "Effects of pretreatment with SKF-525A, N-methyl2-thioimidazole, sodium phenobarbital, or methyl cholanthrene on ethylenethiourea-induced teratogenicity in rats," Teratology 24, 131-137 (1981).

103. B. M. Ulland, J. H. Weisburger, E. K. Weisburger, J. M. Rice, and R. Cypher, "Thyroid cancer in rats from ethylene thiourea intake," J. Natl. Cancer Inst. (1940-1978) 49, 583-584 (1972). 
104. K. S. Khera and F. Iverson, "Toxicity of ethylenethiourea in pregnant cats," Teratology 18, 311-313 (1978).

105. K. S. Khera, "N, N'-Ethylenethiourea. Teratogenicity study in rats and rabbits," Teratology 7, 243-252 (1973).

106. R. A. Weisiger and W. B. Jakoby, "Thiol S-methyltransferase from rat liver," Arch. Biochem. Biophys. 196, 631-637 (1979).

107. R. H. Lindsay, B. S. Hulsey, and H. Y. Aboul-Enein, "Enzymic S-methylation of 6-propyl-2-thiouracil and other antithyroid drugs," Biochem. Pharmacol. 24, 463468 (1975).

108. M. Orban, P. De Kepper, and I. R. Epstein, "Systematic design of chemical oscillators. Part 7. An iodine-free chlorite-based oscillator. The chloritethiosulfate reaction in a continuous flow stirred tank reactor," J. Phys. Chem. 86, 431-433 (1982).

109. I. Nagypal, I. R. Epstein, and K. Kustin, "Systematic design of chemical oscillators. Part 32. Kinetics and mechanism of the reaction between thiosulfate and chlorite ions at 90 Deg," International Journal of Chemical Kinetics 18, 345353 (1986).

110. M. Alamgir and I. R. Epstein, "Systematic design of chemical oscillations. 25. Complex dynamic behavior in a new chemical oscillator: the chlorite-thiourea reaction in a CSTR," Int. J. Chem. Kinet. 17, 429-439 (1985).

111. R. H. Simoyi, M. Manyonda, J. Masere, M. Mtambo, I. Ncube, H. Patel, I. R. Epstein, and K. Kustin, "Systematic design of chemical oscillators. 66. Kinetics and mechanism of the oxidation of thiocyanate by iodate," J. Phys. Chem. 95, 770-774 (1991).

112. M. Orban and I. R. Epstein, "Systematic design of chemical oscillators. Part 13. Complex periodic and aperiodic oscillation in the chlorite-thiosulfate reaction," $\mathrm{J}$. Phys. Chem. 86, 3907-3910 (1982).

113. C. J. Doona, R. Blittersdorf, and F. W. Schneider, "Deterministic chaos arising from homoclinicity in the chlorite-thiourea oscillator," J. Phys. Chem. 97, 72587263 (1993).

114. G. Rabai and I. R. Epstein, "Systematic design of chemical oscillators. 63. Large amplitude $\mathrm{pH}$ oscillation in the oxidation of hydroxylamine by iodate in a continuous-flow stirred tank reactor," J. Phys. Chem. 94, 6361-6365 (1990).

115. M. J. B. Hauser and R. H. Simoyi, "Inhomogeneous precipitation patterns in a chemical wave. Effect of thermocapillary convection," Chem. Phys. Lett. 227, 593-600 (1994). 
116. C. R. Chinake and R. H. Simoyi, "Fingering patterns and other interesting dynamics in the chemical waves generated by the chlorite-thiourea reaction," J. Phys. Chem. 98, 4012-4019 (1994).

117. C. R. Chinake and R. H. Simoyi, "Experimental studies of spatial patterns produced by diffusion-convection-reaction systems," J. Chem. Soc. Faraday Trans. 93, 1345-1350 (1997).

118. R. H. Simoyi, J. Masere, C. Muzimbaranda, M. Manyonda, and S. Dube, "Traveling wave in the chlorite-thiourea reaction," Int. J. Chem. Kinet. 23, 419429 (1991).

119. C. Mundoma and R. H. Simoyi, "Oxyhalogen-sulfur chemistry. Oxidation of 2aminoethanethiosulfuric acid by iodate in acidic medium," J. Chem. Soc. Faraday Trans. 93, 1543-1550 (1997).

120. T. Mizutani and K. Suzuki, "Relative hepatotoxicity of 2-(substituted phenyl)thiazoles and substituted thiobenzamides in mice: Evidence for the involvement of thiobenzamides as ring cleavage metabolites in the hepatotoxicity of 2-phenylthiazoles," Toxicol. Lett. 85, 101-105 (1996).

121. M. A. Salem, C. R. Chinake, and R. H. Simoyi, "Nonlinear Dynamics in Chemistry Derived from Sulfur Chemistry. Part 13. Oxyhalogen-Sulfur Chemistry: Oxidation of Hydroxymethanesulfinic Acid by Chlorite," J. Phys. Chem. 100, 9377-9384 (1996).

122. C. Mundoma and R. H. Simoyi, "Nonlinear dynamics derived from sulfur chemistry. 35. Nonlinear dynamics in the oxidations of substituted thioureas I: the reaction of 4-methyl-3-thiosemicarbazide with acidic iodate," Int. J. Chem. Kinet. 32, 193-203 (2000). 


\section{CHAPTER 2}

\section{Equipment, Techniques, and Materials}

\subsection{INTRODUCTION}

Kinetics is the study and interpretation of the rates of chemical reactions. It does not only deal with rate of a chemical reaction, but also includes factors affecting the rate of the reaction, such as temperature, pressure, ionic strength, solvent, concentrations of reagents etc, and interpretation of the rate in terms of a reaction mechanism [1]. Chemical kinetics tries to answer the question of what happens as the reactants are converted to products. Does the reaction occur in one step, or does it occur in multiple steps via intermediates? The study of chemical kinetics is important especially when one is trying of find the optimum reaction conditions, for example, during organic synthesis and chemical manufacturing.

Having established the overall stoichiometry of the reaction, the first undertaking of any kineticist is to ascertain all the individual steps or elementary reactions and their proper arrangement. Elementary reactions are the simplest obtainable molecular reactions [2], and depending on the number of reacting species can be unimolecular or bimolecular. The collection of all the individual steps in their proper order constitutes what is known as the reaction mechanism [2,3]. This devised model/mechanism may be subject to revision and refinement when new data or new theories are derived.

The rate of reaction is defined as the rate at which the reactant disappears, or the rate at which the product appears. The rate is ascertained by monitoring the concentrations or a certain property of the reagent as a function of time using analytical 
methods. The analytical methods include chemical methods in which one of the reactants or products is measured directly either by volumetric or gravimetric analysis, and physical methods, where a physical property, related to concentration, is measured as a function of time. Chemical methods are able to reveal a conclusive value of concentration directly. Physical methods, although more convenient, rapid, and usually do not result in destruction of the sample, do not furnish the actual concentration directly introducing sources of errors into the measurement. In choosing a physical method, it is important that the products and reactants have significant differences in their contribution to the physical property of choice.

Conventional methods are employed to follow slow reactions with half-lives of several minutes and longer [4]. These methods usually involve mixing of reagents or increasing temperature of the reacting system to start/initiate the reaction prior to taking measurements [5]. The period during which initiation of the reaction occurs, and the time required to make a measurement must be negligible when compared to the half-life of the reaction otherwise errors are introduced into the measurements. Special methods are utilized when dealing with rapid reactions with half-lives of less than a few seconds. Common techniques include: flow methods with stopped-flow $[1,4,6-8]$ being the most common, relaxation techniques [9-12], flash photolysis [13,14], pulse radiolysis [15-18] and magnetic resonance techniques $[19,20]$.

Stopped-flow [21-24] is one of the most frequently utilized rapid kinetics method and incorporates some of the characteristics of both static and flow systems. This technique was first utilized by Chance $[7,6]$ for reactions in solution. The method involves forcing two solutions through jets/syringes into a mixing chamber where rapid 
$\left(<10^{-3} \mathrm{~s}\right)$ mixing occurs. Flow of the solution to the chamber is then stopped suddenly, and data recording commences. The data is processed in much the same way as when using conventional techniques. Stopped-flow techniques have the advantage of using small volumes of reaction solutions, a fact which becomes very important when dealing with biological samples; e.g. enzymes, which are usually obtained in small quantities. This technique is appropriate provided the rate of mixing is fast compared to the rate of reaction being studied [4].

This chapter gives an overview of the instrumentation, materials as well as the experimental methods and techniques used in the elucidation of the oxidation mechanisms of selected thiocarbamides reported in this thesis.

\subsection{INSTRUMENTATION}

\subsubsection{Conventional UV/Vis Spectrophotometry}

Slow reactions, with half-lives of at least several minutes, were monitored using a Perkin Elmer Lambda 2S and a Hewlett-Packard (HP845x UV-Visible Chemstation) spectrophotometer (conventional) within the range of $190-1100 \mathrm{~nm}$. For both instruments, the tungsten lamp was used in the visible region, while the deuterium lamp was used for the ultraviolet (UV) region. Both instruments were interfaced to a Pentium III computer for data acquisition, storage, and manipulation. For the HP spectrometer, HP Biochemical Analysis UV-Vis Software [25] was used to run the instrument, while the Perkin Elmer Computer Spectrophotometer Software (PECSS) [26] was utilized for the Perkin Elmer instrument. For both instruments, the cell path length was $1 \mathrm{~cm}$, and reactions were carried out at a constant temperature of $25{ }^{\circ} \mathrm{C}$. Solutions were first mixed 
using a magnetic stirrer to initiate the reaction before being introduced into the spectrophotometer. A constant time lag of $30 \mathrm{~s}$ was maintained between mixing of reagents and commencement of data acquisition.

\subsubsection{Stopped-Flow Spectrophotometry}

Rapid reactions that could not be monitored using conventional methods, were monitored using stopped-flow techniques $[21,23,22,24]$. This technique allows reactions with time scales of milliseconds to be monitored, and since it has the advantage of rapid mixing, one can obtain experimental points in a very short time scale [5]. A Hi-Tech Scientific SF-61 DX2 double mixing spectrofluorimeter was utilized to monitor experiments with rapid reaction rates. Figure 2.1. is a schematic representation of the instrument, showing all the components of the SF-61 DX2 double mixing spectrofluorimeter [27]. This instrument is essentially a hybrid UV/VIS spectrophotometer designed to monitor fast signal changes using a rapid mixing accessory, the sample handling unit (SHU). The double mixing instrument has the advantage of being able to mix more than two solutions together (multi-mixing) at variable ratios.

Two solutions (each reagent solution containing one of the reactants) contained in separate drive syringes, are rapidly driven into the mixing chamber using a drive motor. Once in the chamber, the solutions are rapidly mixed, thus initiating the reaction, and at the same time displacing the previous aged solution (from the previous experiment) with freshly mixed reactants, which are then driven into the observation cell. The SHU for the SF-61 DX2 instrument facilitates both the single mixing of two reagents using only one 
of the drives, and double mixing three reactants via a push-push mode of operation. The later mode of operation enables transient species formed by mixing, to be subsequently mixed with a third reactant after a set delay period. The stop syringe functions to restrict the volume of solution consumed for each experiment, and to stop the flow. The flow of solution into the stop syringe causes the plunger to move back, subsequently triggering the data acquisition system. The dead time for this instrument is a millisecond, where the dead time is defined as the time it takes for the solution to flow from the mixing chamber to the observation cell. This enables reactions with millisecond and second timescales to be monitored via optical changes using either absorbance or fluorescence detection.

The process of mixing in a stopped-flow apparatus can be considered as occurring in two stages. The mixer takes in two liquids: solution $\mathrm{A}$ and solution $\mathrm{B}$, and partitions each into small volume elements, such that a region of $\mathrm{A}$ is next to a region of $\mathrm{B}$. Mixing is completed by the diffusion of the solute in A into the region of $\mathrm{B}$, and vice versa. Part of this diffusion occurs in the liquid as it flows from the mixer to the observation point, in the dead volume [27]. The more efficient the mixer is, the finer the subdivision into small volumes, and the more rapid the subsequent diffusion. The mixing efficiency is a function of how well the solutions are mixed prior to entering the observation cell.

For more viscous solvents, diffusion will be much slower [28], and consequently mixing will be slower too. Chemical reactions can only occur after the second stage, when reagents $\mathrm{A}$ and $\mathrm{B}$ are in contact at molecular level. This explains the distinctive sigmoidal shape for reaction traces with very rapid reactions rates that are close to the limit of measurability [27]. This is due to the fact that the reagent concentrations will not have reached the acceptable values for complete mixing; hence the reaction occurs at a 
slower rate during the initial millisecond time period. A short dead time as measured above, is not necessarily a good thing. The solution at the observation point may be young, not because it was transported rapidly from the mixer, but because it is still being mixed in the observation cell. Mixing of solutions sets an upper limit for the rate constant obtainable with the instrument. The instrument is designed in a way that ensures that the mixing time is less than the dead time, but not much less. For observed rate constants in excess of $1000 \mathrm{~s}^{-1}$, the experimentally obtained values are actually significantly less than those predicted by linear extrapolation as mixing won't be complete on the time scale under which the reaction occurs [27]. The magnitude of the effect depends upon the nature of the reaction, and the viscosity of the solvent, and has to be independently investigated for each particular system.

The SF-61 DX2 instrument uses proprietary software (Hi-Tech IS 1.0) for data acquisition and analysis, and the program also filters out noise by digitally processing the data. The virtue of this procedure lies in the "digitization error" [27]. The voltage from the photometric circuitry is converted into a digital form suitable for computer processing by a 12-bit analog-to-digital converter. A logarithmic time base is incorporated into the Hi-Tech Scientific IS-1 program; this is particularly powerful in recording complex, multiple exponentials, especially where there a limited quantity of reagent available, since one can span milliseconds to minutes in only one or two runs. With a logarithmic distribution of data points with respect to time, it is possible to achieve a good definition of fast phases, while also achieving good definition of endpoints for slower phases in a single run [29]. 
The light source utilized is a $12 \mathrm{~V}, 50 \mathrm{~W}$ quartz tungsten halogen lamp. The $75 \mathrm{~W}$ super quiet Xenon Arc lamp or a 100W short arc Mercury lamp could also be employed as light sources. A Czerny-Turner type monochromator mounted on a rail is used for this instrument. The instrument had two photomultipliers, an end-on 2 inch Hamamatsu R1463 and a side-on 1-1/8 inch Hamamatsu R928HA. Light passes from the monochromator to the photomultiplier tube via a 600 micron pure silica optic fiber. The optical cell with measurements of $10 \mathrm{~mm} \times 1.5 \mathrm{~mm} \times 1.5 \mathrm{~mm}$ is made from fused UV silica. The cell path length is $10 \mathrm{~mm}$ or $15 \mathrm{~mm}$ depending on the position of the optic fiber.

The diode array [30,31] attachment is a compact multi-channel detector incorporating a linear image sensor [27]. The linear image sensor is a self-scanning photo diode array with a scanning circuit composed of N-channel transistors turned on by a positive gate voltage. In conjunction with the "IBM-PC KINETASYST" software, the Rapid Scan diode array provides a facility for collecting and displaying a sequence of spectrally resolved data which can be manipulated, analyzed, and stored; furthermore cross-sections can be cut through multiple traces to generate time-resolved kinetic traces [27]. 


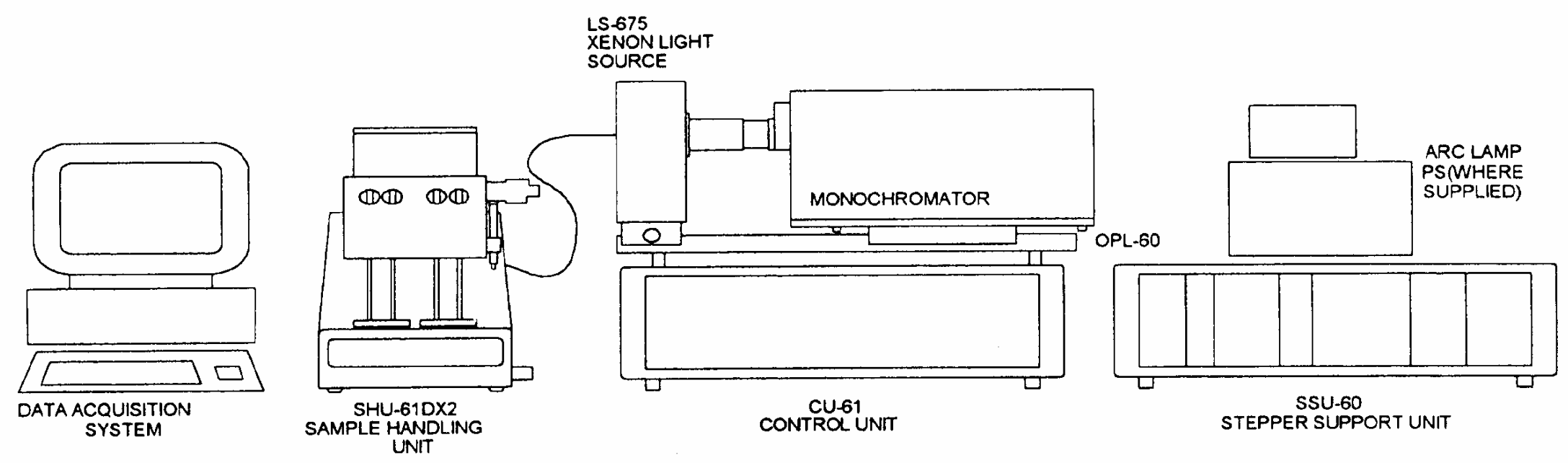

Figure 2.1: Schematic diagram showing all components of the SF61-DX2 double mixing stopped-flow fluorimeter Hi-tech Scientific Operator's Manual [27]. 


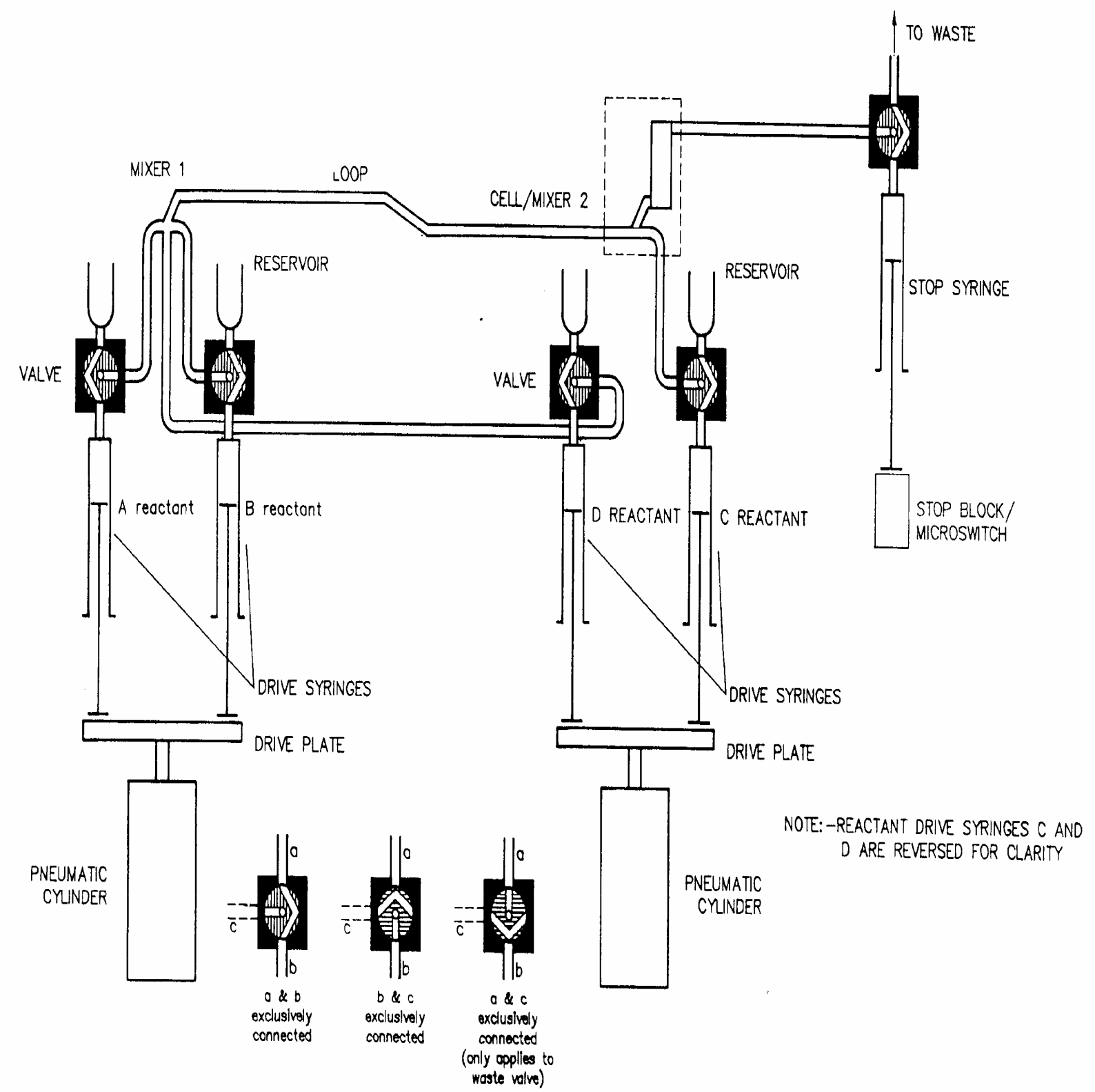

Figure 2.2: Sample Handling Unit (SHU) flow circuit diagram for the SF-61 DX2 double mixing stopped-flow system taken from Hi-tech Scientific Operator's Manual [27]. 


\subsubsection{NMR Spectroscopy}

Structural characterization and verification of products and intermediates was conducted using a JOEL $270 \mathrm{MHz}$ Nuclear Magnetic Resonance (NMR) spectrometer. This was possible as the organosulfur molecules are composed of atoms linked together by bonds. Each one of the atoms possesses a nucleus surrounded by an electron cloud. For the hydrogen atom, the nucleus is composed of a single proton which possesses a property called spin [32]. Nuclei such as the hydrogen nucleus, with unpaired spins of protons and neutrons, will possess an overall nuclear spin, thus creating a nuclear magnetic dipole moment, where the inherent magnitude of the dipole is a nuclear property commonly referred to as the dipole moment, $\mu$. Thus, if the nucleus is placed in a magnetic field, the nuclear dipole moment will precess about the magnetic field. The zcomponent of the nuclear magnetic moment of the nucleus can either be aligned parallel to the external field (low energy) or can be anti-parallel to the external field (high energy). A particle in the low energy state can be excited to the higher energy state, when it absorbs a photon of frequency $v$, where the energy of the photon is the difference in energy between the two states given by [32]

$$
\mathrm{E}=\mathrm{h} v
$$

The variable $v$ is also known as the Larmor frequency or resonance frequency [32,33]. Hence it is the nuclei of atoms that are involved in the absorption process in NMR, unlike in UV/VIS and IR absorption. The signal in NMR spectroscopy is due to the disparities in the energy absorbed by the nuclei as they are excited from the lower energy state to the 
higher energy state, and the energy emitted by the nuclei as they relax from the higher energy state to the lower energy state [33]. The intensity of the signal is proportional to the population difference between the two states; and the area under a given peak is directly proportional to the number of that type of protons in the molecule [34].

The frequency of electromagnetic radiation necessary for resonance to occur depends on both the strength of the external magnetic field, and on the characteristics of the nucleus being studied [33]. The electron cloud surrounding the nucleus tends to shield it from the full strength of the applied magnetic field [32]. The greater the electron density, the greater the shielding, thus nuclei which are in electron-rich environments will undergo transition at a higher applied field than nuclei in electron-poor environments. Similarly, nuclei that are adjacent to electronegative atoms, will be deshielded and undergo transitions at a lower applied filed. The resulting shift in the NMR signal for the given nuclei is referred to as the chemical shift $[34,33]$.

Spin-spin coupling [33] occurs between chemically inequivalent nuclei that are close enough to each other to exert an influence on each other's effective magnetic field, giving rise to the various splitting patterns observed in an NMR spectrum. Such an effect is observable if the distance between the chemically inequivalent nuclei is less than or equal to three bond lengths [33].

In some molecules, certain protons may appear to be inequivalent to other protons in a particular rotamer, although they become equivalent if rapid rotation occurs. In some instances, this does not remove the inequivalence and the protons can be interchanged via symmetry operations. This inherent asymmetry may result in observable differences in the chemical shifts. This phenomenon is best illustrated by the methylene protons of 
$\left(\mathrm{CH}_{3} \mathrm{CH}_{2} \mathrm{O}\right)_{2} \mathrm{SO}$ [34]. The two protons of each of the methylene groups are stereochemically inequivalent due to lack of symmetry at the sulfur center with respect to rotation about the S-O-C bonds. Such inequivalent nuclei, which cannot be interchanged by any of the symmetry elements, are commonly referred to as diastereotopic nuclei.

These phenomena were utilized in monitoring the progress of the reaction during the oxidation of organosulfur compounds. Local symmetry around the sulfur atom, changes during oxidation of an organosulfur compound, with the protons bonded to adjacent $\mathrm{C}$ or $\mathrm{N}$ atoms becoming diastereotropic, or stereochemically equivalent if they were initially diastereotropic.

Unless otherwise stated, the following reagents (Aldrich) and conditions were used: commercial grade deuterated water $\left(\mathrm{D}_{2} \mathrm{O}\right)$, deuterated perchloric acid $\left(\mathrm{DClO}_{4}\right)$ and deuterated sulfuric acid $\left(\mathrm{D}_{2} \mathrm{SO}_{4}\right)$ were used without further purification. All NMR experiments were performed using $\mathrm{D}_{2} \mathrm{O}$ at room temperature. The solvent $\left(\mathrm{D}_{2} \mathrm{O}\right)$ peak, $\delta$ $=4.67$ was used as an internal standard. The reactions were monitored by obtaining spectra at certain time intervals which were determined by the rate of reaction.

\subsection{MATERIALS}

\subsubsection{Oxidants}

The oxidants of choice were chlorite, $\mathrm{ClO}_{2}^{-}$(Fisher), chlorine dioxide, $\mathrm{ClO}_{2}$. Commercially available sodium chlorite (Aldrich) varied in purity (78-88\%) with the main impurities being chloride and carbonate. The sodium chlorite was recrystallized once from a water/ethanol/acetone mixture to bring the assay value to $95 \%$. The recrystallized chlorite was then standardized iodometrically by adding excess acidified 
potassium iodide and titrating liberated iodine against sodium thiosulfate using freshly prepared starch as an indicator [35]. Stock solutions of sodium chlorite were prepared freshly just before use, and were occasionally stabilized using $1.0 \times 10^{-3} \mathrm{M}$ sodium hydroxide especially for reactions conducted in highly acidic conditions.

\subsubsection{Preparation of Chlorine Dioxide}

Chlorine dioxide was prepared by reacting acidified chlorate with oxalic acid [36,37]. The stream was passed through a solution of sodium carbonate to remove impurities, and the chlorine dioxide was then collected in ice-cold water at $4{ }^{\circ} \mathrm{C}$, with $\mathrm{pH}$

adjusted to between $3-4$ using perchloric acid. The solution was stored in stoppered flasks wrapped in aluminium foil and kept at $4{ }^{\circ} \mathrm{C}$ for later use. The stochiometry for the overall reaction is:

$$
2 \mathrm{NaClO}_{3}(\mathrm{~s})+\mathrm{H}_{2} \mathrm{C}_{2} \mathrm{O}_{4} \cdot 2 \mathrm{H}_{2} \mathrm{O}(\mathrm{s})+2 \mathrm{H}_{2} \mathrm{SO}_{4}(\mathrm{aq}) \rightarrow 2 \mathrm{ClO}_{2}(\mathrm{~g})+2 \mathrm{CO}_{2}(\mathrm{~g})+4 \mathrm{H}_{2} \mathrm{O}(\mathrm{l})+2 \mathrm{NaHSO}_{4}
$$

Chlorine dioxide was standardized before use jointly by its molar absorptivity coefficient of $1265 \mathrm{M}^{-1} \mathrm{~cm}^{-1}$ at $360 \mathrm{~nm}$ [37-39] on a Perkin-Elmer Lambda $2 \mathrm{~S} \mathrm{UV} / \mathrm{vis}$ spectrophotometer and iodometrically by adding excess acidified potassium iodide and titrating liberated iodine against sodium thiosulfate using freshly prepared starch as an indicator and employing the following stoichiometries [40]:

$$
\begin{aligned}
2 \mathrm{ClO}_{2}+10 \mathrm{I}^{-}+8 \mathrm{H}^{+} & \rightarrow 5 \mathrm{I}_{2}+2 \mathrm{Cl}^{-}+4 \mathrm{H}_{2} \mathrm{O} \\
\mathrm{I}_{2}+2 \mathrm{~S}_{2} \mathrm{O}_{3}{ }^{2-} & \rightarrow 2 \mathrm{I}^{-}+\mathrm{S}_{4} \mathrm{O}_{6}{ }^{2-}
\end{aligned}
$$




\subsubsection{Organosulfur Compounds}

All the sulfur compounds used for the studies undertaken in this work, as listed in Table 2.1., were of analytical grade and were used without further purification unless otherwise stated.

Table 2.1.: Sulfur compounds used in the studies

\begin{tabular}{llll}
\hline CHEMICAL NAME & FORMULA & SOURCE & ACRONYM \\
\hline 2-aminoethanethiolsulfuric acid & $\mathrm{H}_{2} \mathrm{NCH}_{2} \mathrm{CH}_{2} \mathrm{~S}_{-} \mathrm{SO}_{3} \mathrm{H}$ & Aldrich & AETSA \\
1-Phenyl-2-thiourea & $\mathrm{PhN}(\mathrm{H}) \mathrm{CSNH}_{2}$ & Acros & PTU \\
1,1,3,3-tetramethyl-2-thiourea & $\mathrm{Me}_{2} \mathrm{~N}\left(\mathrm{Me}_{2} \mathrm{~N}\right) \mathrm{C}=\mathrm{S}$ & Acros & TTTU \\
1,1,3-trimethyl-2-thiourea & $\mathrm{Me}_{2}(\mathrm{NHMe}) \mathrm{C}=\mathrm{S}$ & TCI & TMTU \\
\hline
\end{tabular}

\subsubsection{Synthesis of Phenylthiourea dioxide (N-Phenylaminoiminomethanesulfinic acid)}

A mixture of PTU (0.02 moles) and molybdenyl acetylacetone (0.07 moles) dissolved in a 40-60 mixture of ether and dioxane, was slowly added to two equivalents of hydrogen peroxide with continuous stirring at $-15{ }^{0} \mathrm{C}$. The mixture was allowed to stand at room temperature for an hour and left overnight at $-4{ }^{0} \mathrm{C}$. Thawing the resultant mixture, yielded off-white crystals which were then washed using acetonitrile, vacuum dried, enclosed in a dark container, and kept in the freezer for later use.

\subsection{METHODS}

All reactions were run in perchloric acid (72\%, Fisher). Kinetic experiments were carried out at $25.0{ }^{\circ} \mathrm{C}$ and ionic strength was maintained at $1.0 \mathrm{M}$ using analytical grade sodium perchlorate, $\mathrm{NaClO}_{4}$ (Fisher) 


\subsubsection{Reactant and Product Analysis}

\subsubsection{Acid, $\mathrm{H}^{+}$}

Changes in $\mathrm{pH}$ of the solution were detected using acid-base indicators such as methyl red and methyl orange or by use of a $\mathrm{pH}$ electrode.

\subsubsection{Ammonium ions, $\mathrm{NH}_{4}^{+}$}

Ammonium ions were detected by boiling product solutions in strong base and testing the vapor with red litmus paper [39].

\subsubsection{Sulfate Ion, $\mathrm{SO}_{4}{ }^{2-}$}

Sulfate ions were determined by the classical gravimetric method of using barium chloride to precipitate the sulfate as barium sulfate $\left(\mathrm{BaSO}_{4}(\mathrm{~s})\right)$ [41].

\subsubsection{Chloride Ions, $\mathrm{Cl}^{-}$}

Chloride ions were detected by the addition of acidified silver nitrate solution to precipitate the chloride as silver chloride $(\mathrm{AgCl}(\mathrm{s}))$ [39].

\subsubsection{Chlorite Ions, $\mathrm{ClO}_{2}^{-}$}

Excess chlorite ions were determined iodometrically by adding excess acidified potassium iodide and titrating the liberated iodine against sodium thiosulfate [42] using freshly prepared starch as an indicator and utilizing stoichiometries $2.4 \mathrm{a}, 2.3 \mathrm{~b}$ [43,35]:

$$
\mathrm{ClO}_{2}^{-}+4 \mathrm{I}^{-}+4 \mathrm{H}^{+} \rightarrow 2 \mathrm{I}_{2}+\mathrm{Cl}^{-}+2 \mathrm{H}_{2} \mathrm{O}
$$




$$
\mathrm{I}_{2}+2 \mathrm{~S}_{2} \mathrm{O}_{3}{ }^{2-} \rightarrow 2 \mathrm{I}^{-}+\mathrm{S}_{4} \mathrm{O}_{6}{ }^{2-}
$$

\subsubsection{Tests for Adventitious Metal Ion Catalysis}

Water used for preparation of reagent solutions was obtained from a Barnstead Sybron Corporation water purification unit capable of producing both distilled and deionized water (Nanopure). Carrying out the reaction in either distilled water or deionized water did not yield any discernible differences in the overall reaction kinetics. Inductively-Coupled Plasma Mass Spectrometry (ICPMS) analysis was employed in quantifying the metal ion concentrations in the water used for our experimental studies. The results revealed that the water contained insignificant concentrations of iron, copper, and silver and approximately $1.5 \mathrm{ppb}$ of cadimium, and $0.43 \mathrm{ppb}$ of lead. Use of metal chelators, e.g. EDTA, to isolate metal ions, gave overall reaction dynamics that were indistinguishable from those carried out in deionized water and somewhat slower kinetics when compared to reactions done using singly distilled water.

\subsubsection{Stoichiometric Determinations}

Various oxidant to reductant ratios, $\mathrm{R}$, were mixed in stoppered volumetric flasks and scanned spectrophotometrically for formation of chlorine dioxide after a period of two days in order to ascertain the stoichiometry of the reaction. Iodometric techniques were next employed in determining the excess oxidant oxidizing power by adding excess acidified iodide, and then titrating the liberated iodine against standardized sodium thiosulfate. A plot of thiosulfate titer vs. amount of chlorite, when extrapolated to zero titer, yielded the stoichiometry of the reaction. 


\subsubsection{Computer Simulations}

Numerical simulations were carried out to complement our experiments on the oxidation of organosulfur compounds. After deriving a postulated reaction mechanism from experimental data, and using it to formulate a model for the reaction such that it closely parallels the experimental design, the next step for a kineticist is to check the accuracy of the model [1]. For simple systems, the model can be tested by applying certain approximations to the differential equations, such as steady-state approximations, quasi-state hypothesis or stationary-state hypothesis [1]. Differential equations for complex mechanisms on the other hand are solved using numerical methods/simulations which yield values of concentration as a function of time. The model is a list of all the elementary steps involved in the reaction in the correct sequence. It involves the reaction mechanism, with appropriate rate constants and a set of parameter values describing the initial conditions which are similar to those used in the experiment. From the model, a set of differential equations can be obtained showing the time dependence of each of the chemical variables. Prior to carrying out the simulations, certain approximations are applied to the differential equations in an attempt to simplify and/or combine them [1].

Solving the set of differential equations, gives the concentration of the chemical variables as a function of time. The simulated traces can then be compared to the experimental traces, and usually certain variables like rate constants or initial conditions are altered until the simulated traces are comparable to the experimental traces. There are two major techniques available for carrying out the simulations [44], namely the deterministic approach [45] and stochastic approach [46]. In the first approach, the timedependent behavior of the system is both continuous and deterministic, being regulated 
by the set of coupled ODE's [45]. The second approach on the other hand, is a much simpler one which considers the time-dependent behavior of the system as a discrete, random process determined by a single differential-difference equation $[45,46]$. The Runge-Kutta method [47] is commonly used to integrate a set of coupled ordinary differential equations (ODE's). When using this method, one assumes that the reaction is sufficiently well-understood and that the overall time-dependent behavior of the system is contained in the solution of the differential equations. This method is efficient in solving the differential equations although one encounters problems when a large number of steps are involved in the reaction mechanism [1].

The Chemical Kinetics Simulator (CKS) software package developed by IBM's Almaden Research Center [44] and KINTECUS developed by James C. Ianni [48] were used for carrying out simulations in this study. Both these algorithms are as accurate as the semi-implicit fourth order Runge-Kutta scheme developed by Kaps and Rentrop [49], and require fewer computer resources. CKS is a fairly user friendly package requiring no programming skills by the user. Reactions are simulated using a stochastic approach rather than the usual numerical solution of differential equations, and it calculates the concentration of all reactants as a function of time [44]. Instead of obtaining a solution describing the continuous behavior of the system in time, a probabilistic method for predicting the time evolution of the reacting system is employed. Figure 2.3. shows an illustration of the basic simulation loop used in the program [44]. The stochastic method is highly accurate as it takes full account of fluctuations and correlations as implied by the fundamental hypothesis of the stochastic formulation. CKS, however, is not capable 
of automatically finding a mechanistic fit to the experimental data, and often requires lots of computer time to execute.

KINTECUS on the other hand is powerful, easy to use, and is a rapid chemical kinetics compiler capable of carrying out simulations on a wide variety of chemical systems [48]. Unlike the CKS program, KINTECUS can actually fit the parameters at exactly the same time scale as the experimental data, and is able to use data from other programs such as Chemkin, SENKIM II/III without supercomputing knowledge, compiling or linking required. The program uses three input spreadsheet files: a reaction spreadsheet, a species description spreadsheet and a parameter description spreadsheet. An optional thermodynamics spreadsheet can be supplied for thermodynamic simulations. 


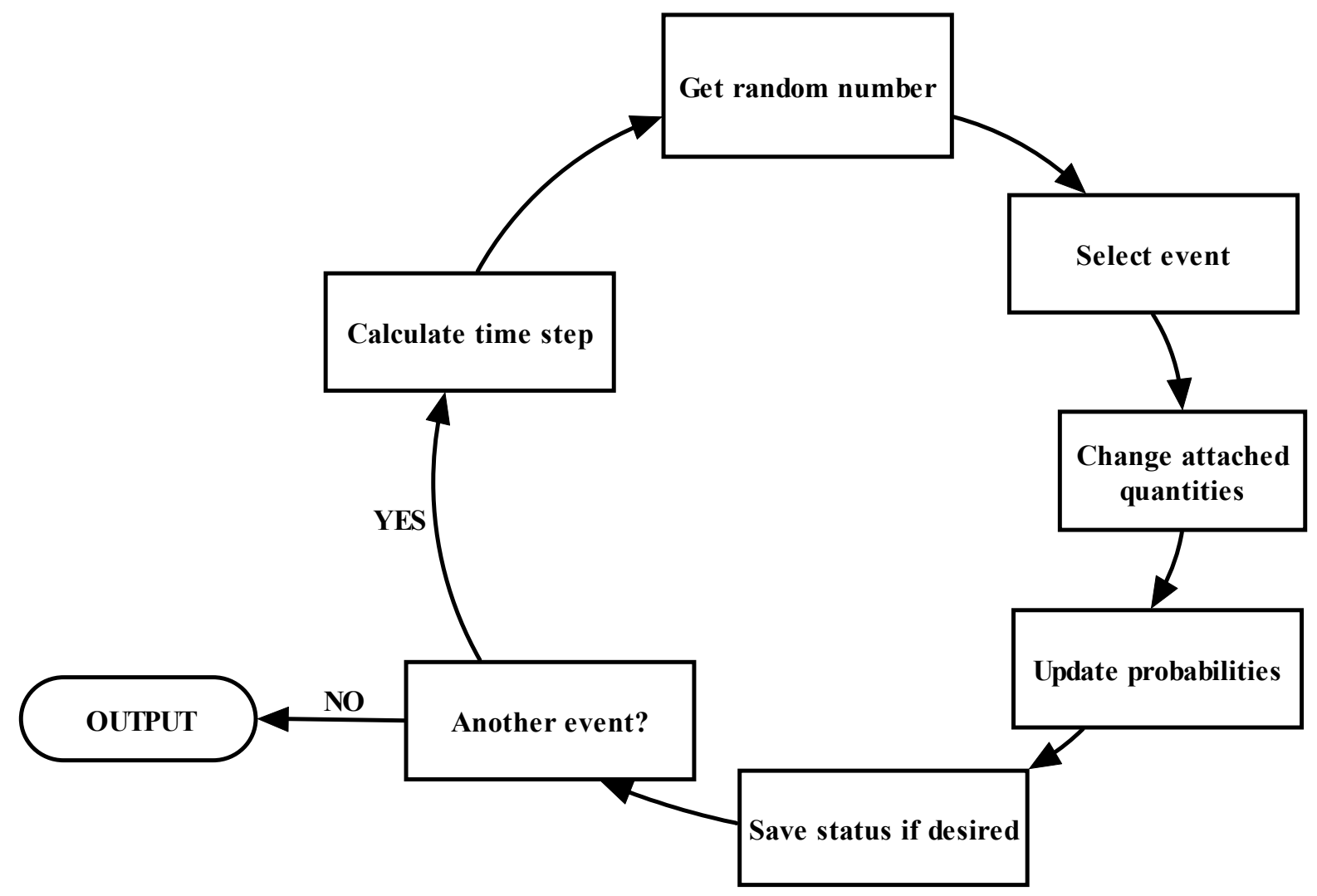

Figure 2.3: System propagation routine in the stochastic algorithm employed by CKS [44]. 


\section{References}

1. J. H. Espenson, Chemical Kinetics and Reaction Mechanisms, (McGraw-Hill, New York, 2002).

2. K. A. Connors, Chemical Kinetics: The Study of Reaction Rates in Solution, (VCH, New York, 1990).

3. M. Boudart, Kinetics of Chemical Processes, (Butterworth-Heinemann, Boston, 1991).

4. K. J. Laidler, Chemical Kinetics, (McGraw-Hill, New York ,1965).

5. A. A. Frost, R. G. Pearson, and a. joint, Kinetics and Mechanism; a Study of Homogeneous Chemical Reactions, (New York, Wiley, 1961).

6. B. Chance, "The accelerated-flow method for rapid reactions. II. Design, construction and tests," J. Franklin I. 229, 737-766 (1940).

7. B. Chance, "The accelerated-flow method for rapid reactions. I. Analysis," J. Franklin I. 229, 455-76,613 (1940).

8. Y. Engelborghs and K. Heremans, "Recent applications of the stopped-flow and pressure-jump relaxation techniques in the biological sciences," Biochem. Cell Biol. 39-62 (1983).

9. D. Poerschke, "Methods for studying fast kinetics in biological systems," NATO Advanced Study Institute Series, Series A: Life Sciences 45, 333-358 (1982).

10. G. M. Schneider, M. Dittmann, U. Metz, and J. Wenzel, "Kinetics of phase separation in fluid mixtures by relaxation methods: pressure-jump experiments and selected results," Pure Appl. Chem. 59, 79-90 (1987).

11. H. Strehlow, "Relaxation techniques in fast reaction kinetics," Adv. Mol. Relax. Pr. 2, 235-249 (1972).

12. H. Winkler, "Recent progress in the study of fast chemical reactions by relaxation techniques," Endeavour 33, 73-79 (1974).

13. T. J. Kemp, "Kinetic aspects of spin trapping," Prog. React. Kinet. Mec. 24, 287358 (1999).

14. J. Wirz, "Getting slow-motion pictures of fast reactions," Chimia 53, 232-234 (1999).

15. R. Van Eldik and D. Meyerstein, "High-pressure pulse radiolysis as a tool in the study of transition metal reaction mechanisms," Acc. Chem. Res. 33, 207-214 (2000). 
16. S. Adhikari and T. Mukherjee, "Kinetics of free radical reactions of some biologically important compounds as studied by pulse radiolysis," Prog. React. Kinet. Mec. 26, 301-336 (2001).

17. Y. Katsumura, "Ion beam pulse radiolysis study on intra-track reactions in aqueous solutions," Res. Chem. Intermediat. 27, 333-341 (2001).

18. P. Neta, D. Behar, and J. Grodkowski, "Pulse radiolysis studies of reaction kinetics in ionic liquids," ACS Symposium Series 856, 397-409 (2003).

19. A. G. Palmer, III, C. D. Kroenke, and J. P. Loria, "Nuclear magnetic resonance methods for quantifying microsecond-to-millisecond motions in biological macromolecules," Methods Enzymol. 339, 204-238 (2001).

20. G. Haneczok and J. Rasek, "Some applications of mechanical spectroscopy and magnetic relaxation techniques to the examination of diffusion processes in metals and alloys," Defect Diffus. Forum 188-190, 3-20 (2001).

21. L. Simandi, "New developments in the stopped-flow technique for fast reactions in solution," Magy. Kemik. Lapja 57, 122-126 (2002).

22. J. F. Eccleston, J. P. Hutchinson, and H. D. White, "Stopped-flow techniques," in Protein-Ligand Interactions, Structure and Spectroscopy: A Practical Approach, edited by S.E Harding and B.Z Chowdry, (Oxford University press, Oxford, 2001) p. 201-237.

23. K. Hiromi, "New fields of application of the stopped-flow technique," Trends Biochem. Sci. 3, 232-235 (1978).

24. A. Gomez-Hens and D. Perez-Bendito, "The stopped-flow technique in analytical chemistry," Anal. Chim. Acta 242, 147-177 (1991).

25. HP Operator's Manual for the HP8453 Spectrophotometer, (Hewlett Packard Inc, USA, 1987).

26. Perkin Elmer Lambda2 UV/VIS Spectromether User Manual, (Perkin Elmer, USA, 1992).

27. Hi-Tech Scientific Operator's Manual for the SF-61 DX2 Double-Mixing StoppedFlow Spectroflurieter, (Hi-Tech Limited, Salisbury, 1996).

28. M. J. Pilling and P. W. Seakins, Reaction Kinetics, (Oxford University Press, Oxford, 1995).

29. A. R. Walmsley and C. R. Bagshaw, "Logarithmic timebase for stopped-flow data acquisition and analysis," Anal. Biochem. 176, 313-318 (1989). 
30. D. G. Jones, "Photodiode array detectors in UV-VIS spectroscopy: Part I," Analytical Chemistry 57, 1057A-1058A, 1060A, 1062A, 1064A, 1066A, 1068A, 1070A, 1073A (1985).

31. D. G. Jones, "Photodiode array detectors in UV-VIS spectroscopy: part II," Anal. Chem. 57, 1207A-1208A (1985).

32. D. A. Skoog, Principles of Instrumental Analysis, (Saunders College Pub., Philadelphia, 1998).

33. R. Freeman, A Handbook of Nuclear Magnetic Resonance, (Longman Scientific \& Technical, Essex, 1987).

34. R. S. Drago and R. S. Drago, Physical Methods for Chemists, (Saunders College Pub., Fort Worth, 1992).

35. A. Indelli, "Kinetic study on the reaction of sodium chlorite with potassium iodide," J. Phys. Chem. 68, 3027-3031 (1964).

36. G. Brauer, Handbook of Preparative Inorganic Chemistry, Vol. 1. (Academic Press, New York, 1975) $3^{\text {rd }}$ Ed.

37. G. Gordon, R. G. Kieffer, and D. H. Rosenblatt, "Chemistry of chlorine dioxide," Prog. Inorg. Chem. 15, 201-286 (1972).

38. I. Lengyel, G. Rabai, and I. R. Epstein, "Experimental and modeling study of oscillations in the chlorine dioxide-iodine-malonic acid reaction," J. Am. Chem. Soc. 112, 9104-9110 (1990).

39. J. S. Clarke, Advanced Practical Chemistry, (Hodder and Stoughton, London, 1979).

40. J. Darkwa, R. Olojo, E. Chikwana, and R. H. Simoyi, "Antioxidant chemistry: oxidation of L-cysteine and its metabolites by chlorite and chlorine dioxide," J. Phys. Chem. A 108, 5576-5587 (2004).

41. D. A. Skoog and D. M. West, Analytical Chemistry, (Saunders College Pub., New York, 1980).

42. R. H. Simoyi, "Autocatalytic chlorite-bromide reaction," J. Phys. Chem. 89, 35703574 (1985).

43. D. M. Kern and C. H. Kim, "Iodine catalysis in the chlorite-iodide reaction," J. Am. Chem. Soc. 87, 5309-5313 (1965).

44. Chemical Kinetics Simulator User's Manual, (IBM Corporation, USA, 1995). 
45. J. S. Turner, "Discrete simulation methods for chemical kinetics," J. Phys. Chem. 81, 2379-2408 (1977).

46. D. T. Gillespie, "Exact stochastic simulation of coupled chemical reactions," J. Phys. Chem. 81, 2340-2361 (1977).

47. W. H. Press, Numerical Recipes in C: The Art of Scientific Computing, (Cambridge University Press, Cambridge, 2002).

48. I. C. James, KINTECUS User's Manual, (Vast Technologies Development Inc, Lansdowne, 1995).

49. P. Kaps and P. Rentrop, "Application of a variable-order semi-implicit Runge-Kutta method to chemical models," Comput. Chem. Eng. 8, 393-396 (1985). 


\section{CHAPTER 3}

\section{Oxidation of Tetramethylthiourea by Chlorite in Acidic Media}

\subsection{INTRODUCTION}

Tetramethylthiourea, TTTU, a member of the group of compounds called thiocarbamides, is known to be a chemical teratogen [1], carcinogen [2], mutagen, that possesses strong antithyroid activity in rats [3]. TTTU is a fetotoxin that produces malformations in the tail, palate or extremities in surviving rat fetuses [1]. Like dimethylthiourea, it is a potent active oxidant scavenger (AOS) [4-7], and has been reported to prevent active wall fibrosis, hence chronic obstructive pulmonary disease due to air pollutant particles (dust) [5]. None of the other substituted thioureas are as potent. Thioureas, in general, differentially induce physiological effects based on their structures i.e. degree and type of substituents attached to the parent thiourea, suggesting differences in their bioactivation mechanisms.

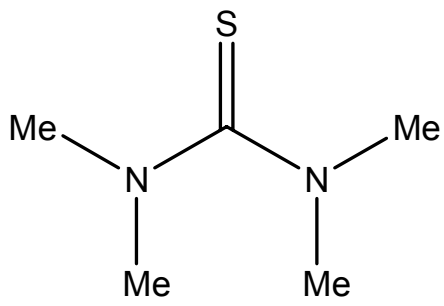

Tetramethylthiourea

This work is part of the continued efforts in trying to elucidate the S-oxygenation mechanisms of a series of substituted thioureas. This chapter reports on the comprehensive study on the oxidation mechanism of TTTU by mildly acidic chlorite solutions. TTTU differs from other substituted thioureas in that it does not possess a 
detachable proton on any of the nitrogen groups, and thus is unable to form stable oxoacids such as sulfinic and sulfonic acids without resorting to the formation of a zwitterion. These zwitterions are extremely unstable especially in the presence of nucleophiles in which a nucleophilic attack on the carbon center of the $\mathrm{CN}_{2}$ unit is very facile. Our preliminary investigations have shown that the reaction dynamics presented by the oxidation of TTTU are very different from those observed for the oxidation of dimethylthiourea, and trimethylthiourea using similar oxidants.

\subsection{RESULTS}

\subsubsection{Stoichiometry}

In excess chlorite conditions, the stoichiometry of the reaction was determined to be:

$2 \mathrm{ClO}_{2}{ }^{-}+\mathrm{Me}_{2} \mathrm{~N}\left(\mathrm{Me}_{2} \mathrm{~N}\right) \mathrm{C}=\mathrm{S}+\mathrm{H}_{2} \mathrm{O} \rightarrow 2 \mathrm{Cl}^{-}+\mathrm{Me}_{2} \mathrm{~N}\left(\mathrm{Me}_{2} \mathrm{~N}\right) \mathrm{C}=\mathrm{O}+\mathrm{SO}_{4}{ }^{2-}+2 \mathrm{H}^{+}$

This stoichiometry was determined by a combination of spectrophotometric, titrimetric, and gravimetric techniques. Figure 3.1 shows three superimposed spectra; (a) tetramethylurea, $\mathrm{TMU}\left(\lambda_{\max }=206 \mathrm{~nm}, \epsilon_{\max }=38,153 \mathrm{M}^{-1} \mathrm{~cm}^{-1}\right.$, and $€=0 \mathrm{M}^{-1} \mathrm{~cm}^{-1}$ at $\lambda=$ $248 \mathrm{~nm})$, (b) tetramethylthiourea, TTTU $\left(\lambda_{\max }=248 \mathrm{~nm}, \epsilon_{\max }=17,556 \mathrm{M}^{-1} \mathrm{~cm}^{-1}\right)$, and (c) chlorine dioxide $\left(\epsilon=135 \mathrm{M}^{-1} \mathrm{~cm}^{-1}\right.$ at $\left.\lambda=248 \mathrm{~nm}\right)$. Chlorine dioxide has negligible absorbance at $248 \mathrm{~nm}$ as shown by the low absorptivity coefficient. Tetramethylurea on the other hand, does not contribute at all to the absorbance at $248 \mathrm{~nm}$. 


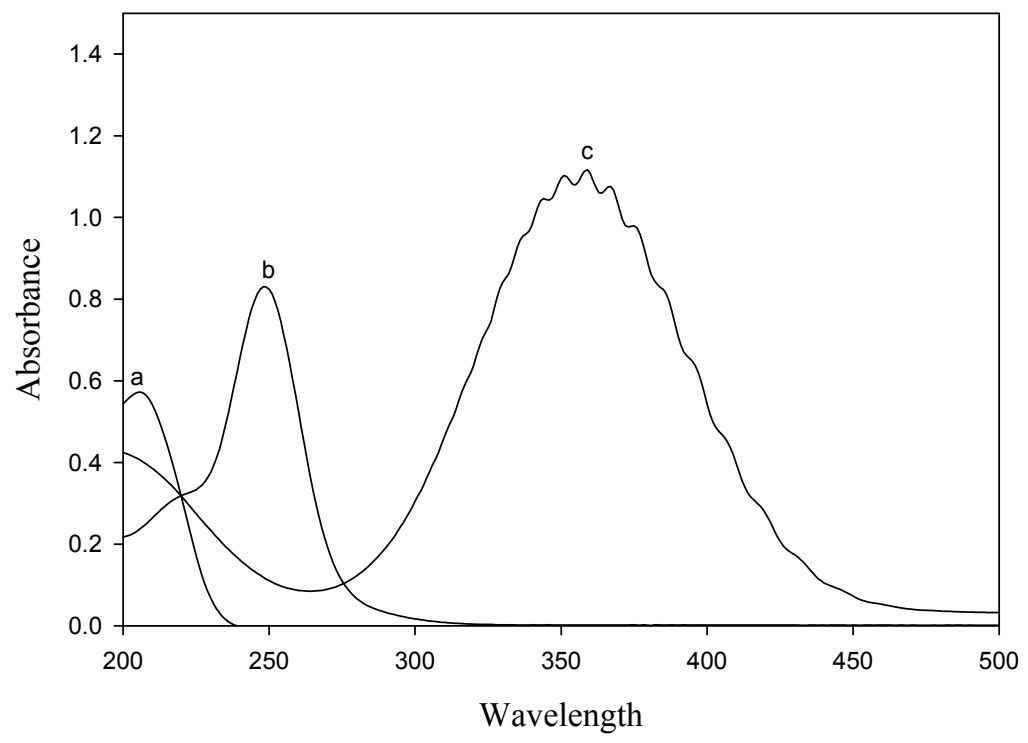

Figure 3.1: UV absorption spectrum of (a) $1.500 \times 10^{-5}$ Tetramethlyurea (b) $5.000 \times 10^{-5} \mathrm{M}$ TTTU and (c) $8.758 \times 10^{-4} \mathrm{M} \mathrm{ClO}_{2}$ showing peaks at $206 \mathrm{~nm}, 248 \mathrm{~nm}$ and $360 \mathrm{~nm}$ respectively. There is no interference from the substrate or product at $360 \mathrm{~nm}$.

The stoichiometric ratio, $\mathrm{R}$, needed for reaction 3.1 was the highest amount of chlorite needed for a fixed amount of TTTU that did not give the yellow chlorine dioxide as a product (excluding spurious and/or transient chlorine dioxide formations). Figure 3.3a shows the effect of altering initial chlorite concentrations (effectively altering R), while keeping all the other species constant. In this series of experiments, any chlorite concentrations with $\mathrm{R}<2$, did not produce chlorine dioxide and hence could not show an induction period (see figure $3 b$ ). Such traces will have an infinitely long induction period, and the inverse of such an induction period will be zero. An extrapolation of the linear plot of inverse of induction period vs. chlorite concentrations will give the exact amount of chlorite needed for stoichiometry 3.1. For a fixed amount of TTTU, $0.00125 \mathrm{M}$, the intercept value of chlorite obtained was $0.002547 \mathrm{M}$ (see figure $3.3 \mathrm{~b}$ ); which is very close to stoichiometry $2: 1$ of reaction 3.1 . 


\subsubsection{Reaction dynamics}

Figure 3.2 shows the observed activity of the reaction in the UV region. Initially there is a decrease in the peak at $248 \mathrm{~nm}$, while no activity is observed at $360 \mathrm{~nm}$. After a short induction time, a peak which increases in height is observed at $360 \mathrm{~nm}$, while the peak at $248 \mathrm{~nm}$ continues to decrease in height. The final absorption reading at $360 \mathrm{~nm}$ is attained after a very long incubation period, up to days in some cases. Most of the rapid increase in the peak at $360 \mathrm{~nm}$ is accomplished within the first $5-10 \mathrm{~s}$. Further increase after this period is very slow, and the chlorine dioxide formed after this period is due to the disproportionation of chlorite solutions in acidic media [8]:

$$
5 \mathrm{ClO}_{2}^{-}+4 \mathrm{H}^{+} \rightarrow 4 \mathrm{ClO}_{2(\mathrm{aq})}+2 \mathrm{H}_{2} \mathrm{O}+\mathrm{Cl}^{-}
$$

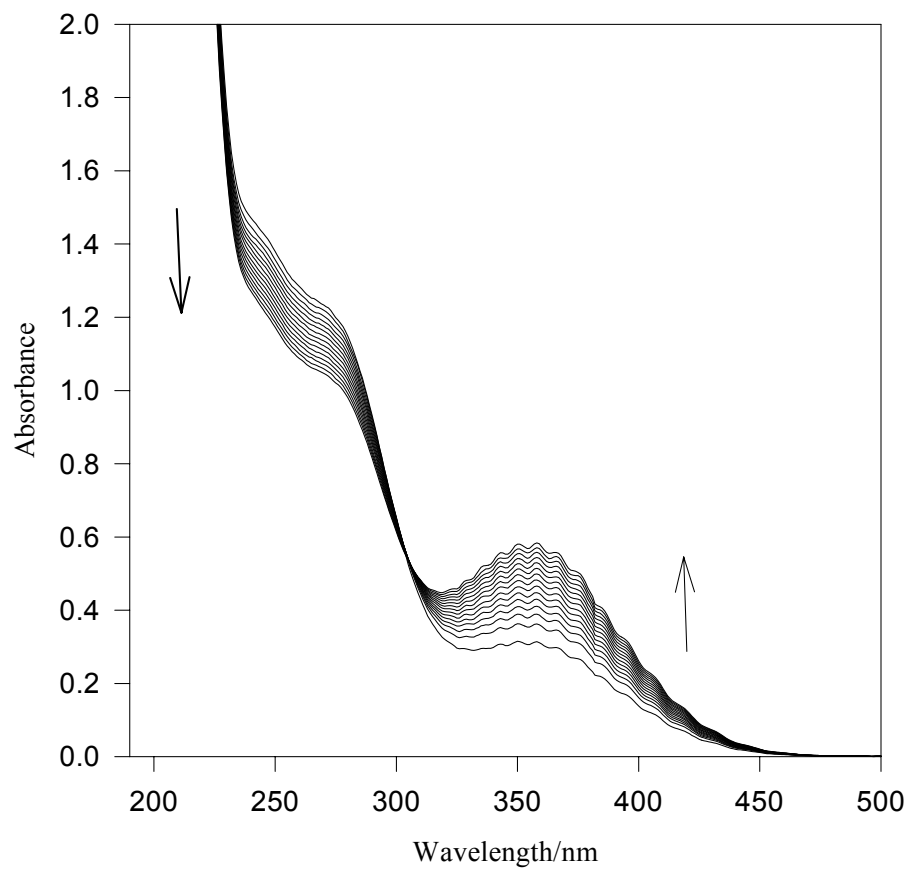

Figure 3.2: Absorbance scans of TTTU during oxidation by chlorite in aqueous acidic medium. Traces were collected every 60 second reaction time intervals. $[\mathrm{TTTU}]_{0}=5 \times 10^{-4} \mathrm{M},\left[\mathrm{ClO}_{2}^{-}\right]_{0}=4$ x $10^{-3} \mathrm{M}$, and $\left[\mathrm{HClO}_{4}\right]_{0}=0.1 \mathrm{M}$. 


\subsubsection{Chlorite dependence}

Figures $3.3 \mathrm{a}$ and $3.3 \mathrm{~b}$ show that there in an inverse relationship (to the first power) between the induction period and initial chlorite concentrations. Such a correlation would indicate that the reactions that consume TTTU, and those that form chlorine dioxide, though they both involve chlorite, react at different time scales. A ratio of oxidant to reductant of least 4.0 ensures that all the TTTU is oxidized to TMU. If the reaction between the product chlorine dioxide, and TTTU is slow, then one would expect oligooscillatory behavior in chlorine dioxide production.

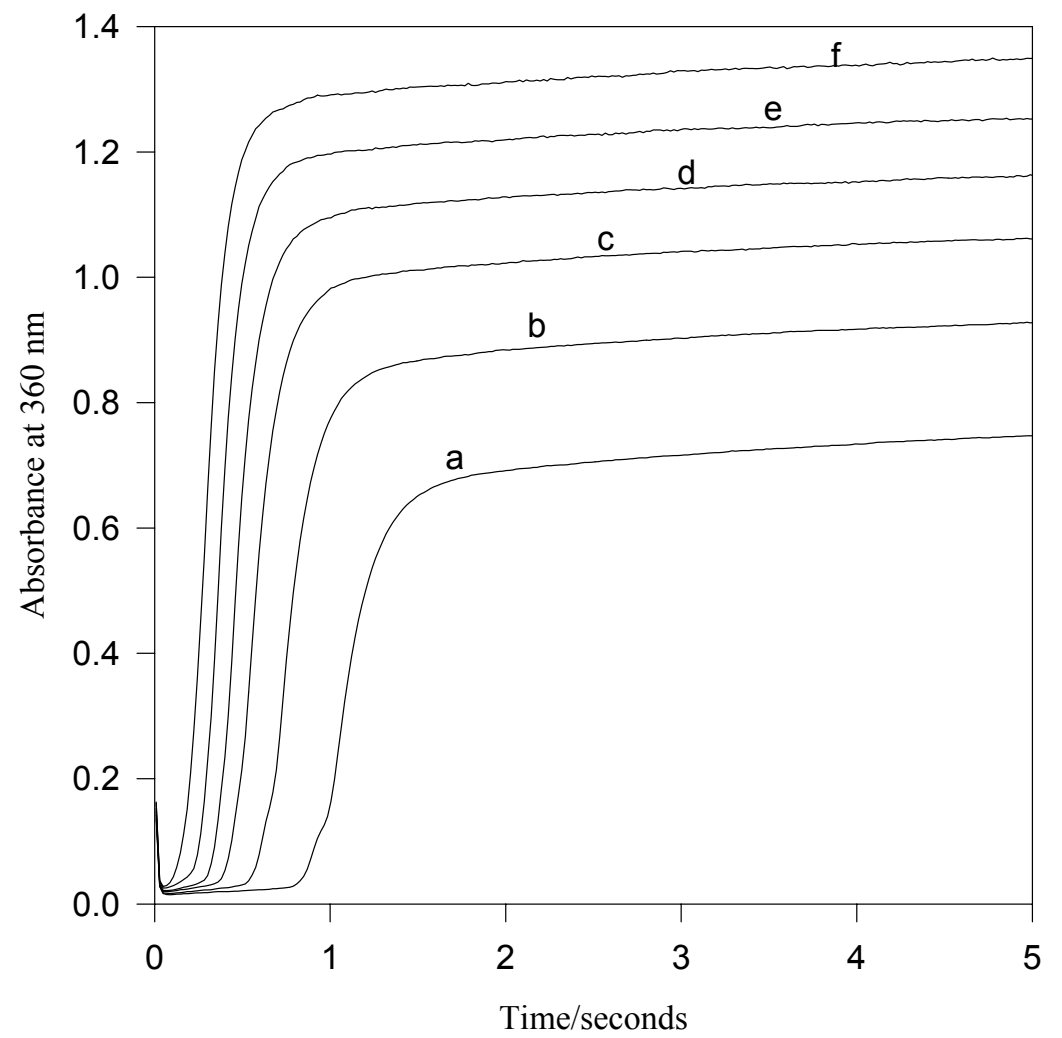

Figure 3.3a: Effect of chlorite on the absorbance at $360 \mathrm{~nm}$. Concentration of chlorite is directly proportional to the amount of $\mathrm{ClO}_{2}$ formed and inversely proportional to the induction period at fixed TTTU concentrations: $[\text { TTTU }]_{0}=1.25 \times 10^{-3} \mathrm{M},\left[\mathrm{HClO}_{4}\right]_{0}=0.125 \mathrm{M}, \mathrm{I}_{(\mathrm{NaClO} 4)}=1 \mathrm{M},\left[\mathrm{ClO}_{2}{ }^{-}\right.$ ]$_{0}=$ (a) $5.00 \times 10^{-3} \mathrm{M}$, (b) $6.25 \times 10^{-3} \mathrm{M}$, (c) $7.50 \times 10^{-3} \mathrm{M}$, (d) $8.75 \times 10^{-3} \mathrm{M}$, (e) $=10.00 \times 10^{-3} \mathrm{M}$, (f) $11.25 \times 10^{-3} \mathrm{M}$. 


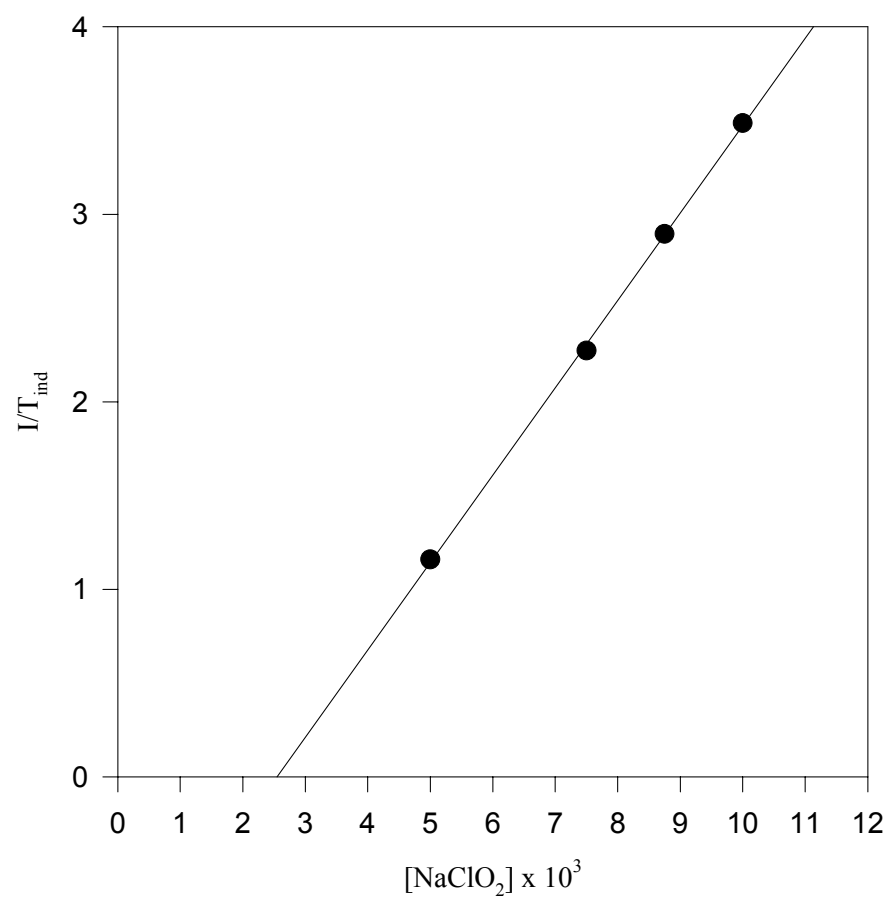

Figure 3.3b: Plot of $1 /$ Tind vs $\left[\mathrm{ClO}_{2}^{-}\right]$using stochiometric excess of chlorite, showing that an inverse relationship exists for a long range of chlorite concentrations. [TTTU $]_{0}=1.25 \times 10^{-3} \mathrm{M}$, $\left[\mathrm{HClO}_{4}\right]_{0}=0.125 \mathrm{M}, \mathrm{I}_{(\mathrm{NaClO} 4)}=1 \mathrm{M}$.

\subsubsection{Acid dependence}

The effect of acid depends on its strength. Figure 3.4a shows the effect of acid in low acid environments $(0.005-0.025 \mathrm{M})$. There is no real discernible induction period under these conditions ( $\mathrm{cf}$ figure $3.3 \mathrm{a}$ with $\left[\mathrm{H}^{+}\right]_{0}=0.125 \mathrm{M}$ ). In general, however, there is an increase in the rate of formation of chlorine dioxide with increase in acid concentrations for this acid range. A reversal of this trend is observed in high acid environments as shown in figure 3.4b. A well defined induction period which is independent of acid concentrations is now observed, although there is a decrease in the rate and amount of chlorine dioxide formed as acid concentrations are increased. This behavior could be due to the fact that acid either retards the reactions that form chlorine dioxide or catalyzes the reactions that consume it. 


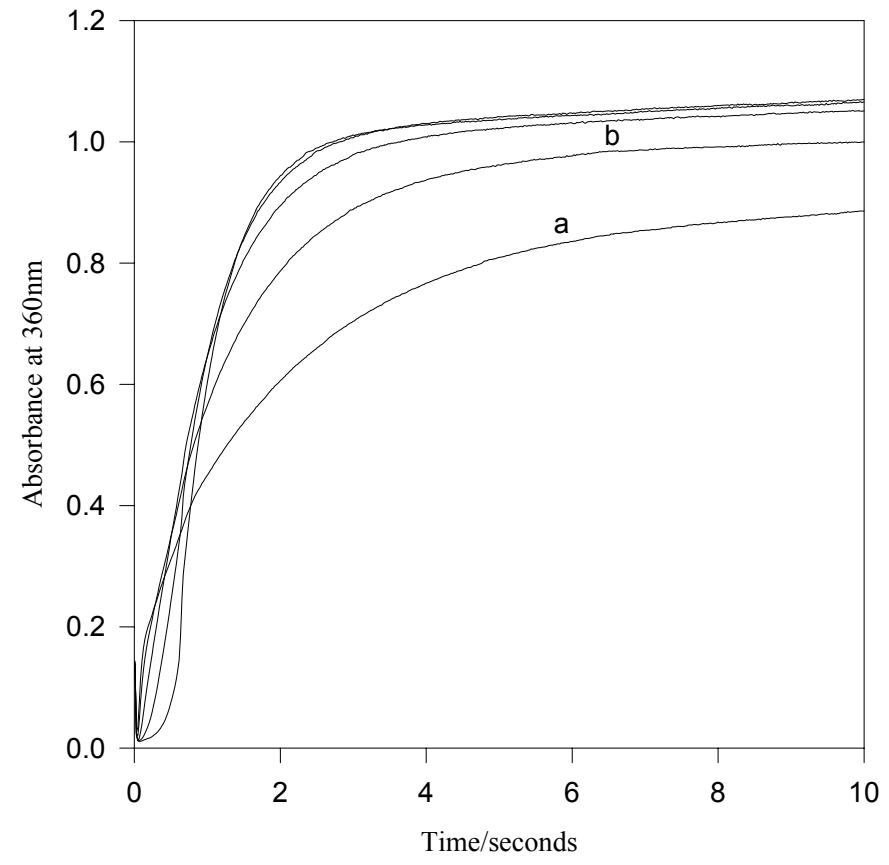

Figure 3.4a: Absorbance traces at $360 \mathrm{~nm}$ showing effect of low acid concentrations on the oxidation of TTTU by chlorite. In this case there is no well defined induction period. [TTTU $]_{0}=$ $1.25 \times 10^{-3} \mathrm{M},\left[\mathrm{ClO}_{2}^{-}\right]_{0}=5.00 \times 10^{-3} \mathrm{M}, \mathrm{I}_{(\mathrm{NaClO} 4)}=1 \mathrm{M},\left[\mathrm{HClO}_{4}\right]_{0}=$ (a) $0.005 \mathrm{M}$, (b) $=0.010 \mathrm{M}$.

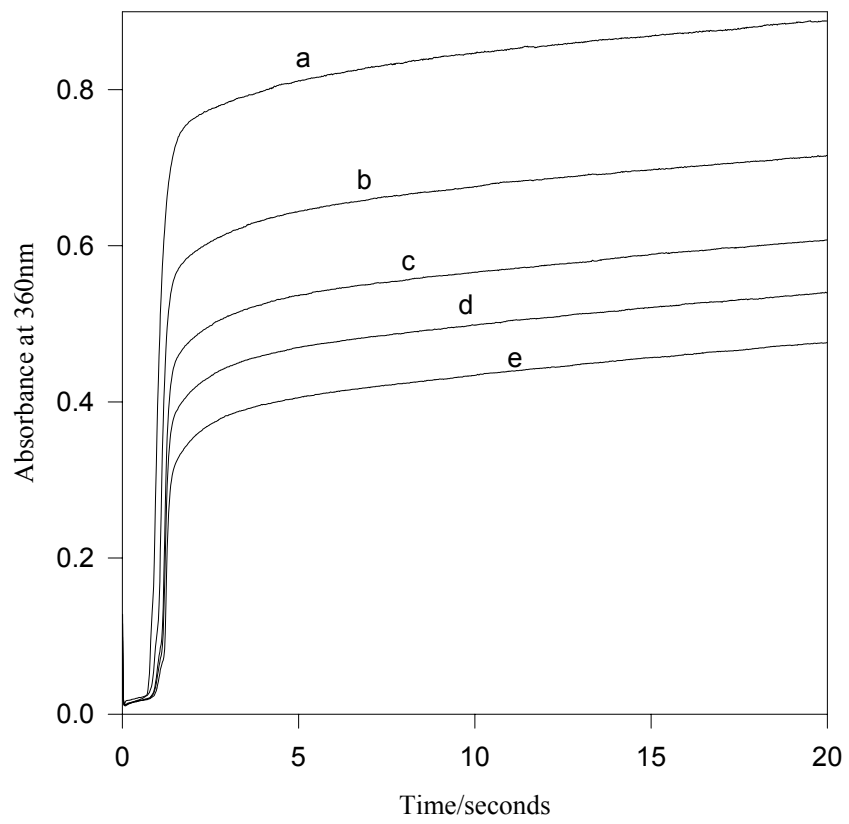

Figure 3.4b: Absorbance traces at $360 \mathrm{~nm}$ showing effect of high acid concentrations on the oxidation of TTTU by chlorite. In this case there is a well defined induction period which in not affected by the amount of acid. Concentration of acid is inversely proportional to the amount of chorine dioxide formed. The amount of $[\mathrm{TTTU}]_{0}=1.25 \times 10^{-3} \mathrm{M},\left[\mathrm{ClO}_{2}\right]_{0}=5.00 \times 10^{-3} \mathrm{M}, \mathrm{I}_{(\mathrm{NaClO} 4)}$ $=1 \mathrm{M},\left[\mathrm{HClO}_{4}\right]_{0}=$ (a) $0.1 \mathrm{M}$, (b) $0.2 \mathrm{M}$, (c) $0.3 \mathrm{M}$, (d) $0.4 \mathrm{M}$, (e) $0.5 \mathrm{M}$. 


\subsubsection{TTTU dependence}

The effect of TTTU shown in figure 3.5 is expected for conditions in which the chlorite is in overwhelming excess over TTTU. There are very small variations in the induction period with variations in TTTU concentrations. Higher TTTU concentrations, however, will show higher and more rapid production of chlorine dioxide after the induction period.

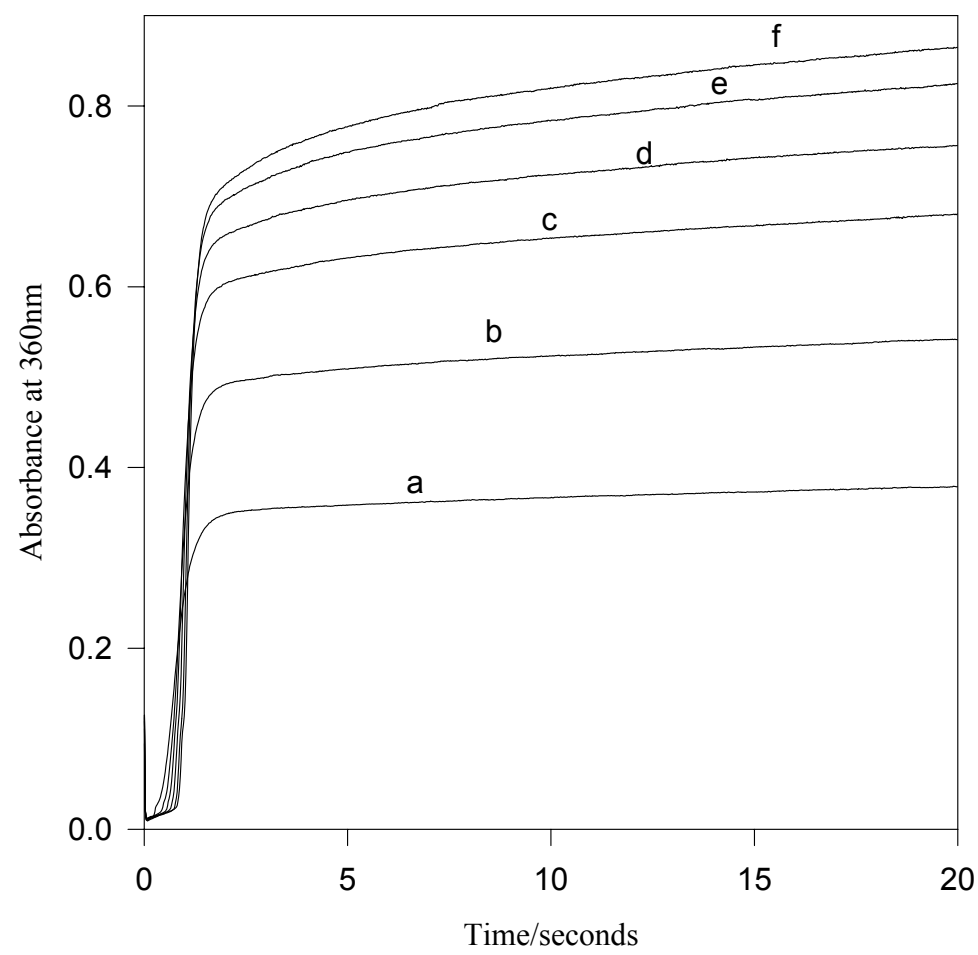

Figure 3.5: "Peacock-tail type" traces derived from variation of [TTTU $]_{0}$ in large excess of $\left[\mathrm{ClO}_{2}{ }^{-}\right]_{0}$. The induction period stays the same although the rate of formation of chlorine dioxide increases. The amount of chlorine dioxide formed is directly proportional to the amount $[\mathrm{TTTU}]_{0}$. $\left[\mathrm{ClO}_{2}{ }^{-}\right]_{0}=5.00 \times 10^{-3} \mathrm{M},\left[\mathrm{HClO}_{4}\right]_{0}=0.125 \mathrm{M}, \mathrm{I}_{(\mathrm{NaClO} 4)}=1 \mathrm{M},[\mathrm{TTTU}]_{0}=$ (a) $2.5 \times 10^{-4} \mathrm{M}$, (b) 5.0 x $10^{-4} \mathrm{M}$, (c) $7.5 \times 10^{-4} \mathrm{M}$, (d) $10.0 \times 10^{-4} \mathrm{M}$, (e) $12.5 \times 10^{-4} \mathrm{M}$, (f) $15.0 \times 10^{-4} \mathrm{M}$.

\subsubsection{Consumption of TTTU}

Figure 3.6a shows absorbance traces at $248 \mathrm{~nm}$, the $\lambda_{\max }$ for TTTU. It shows a two-phase reaction progress in which the first stage is characterized by a rapid decrease 
in absorbance followed by a stage where there is no change in absorbance. The second stage involved a rapid autocatalytic drop in absorbance to the final residual absorbance arising mainly from chlorine dioxide. The initial phase of this reaction has first order dependence on chlorite concentrations. The rate of the second phase of the reaction is also catalyzed by chlorite.

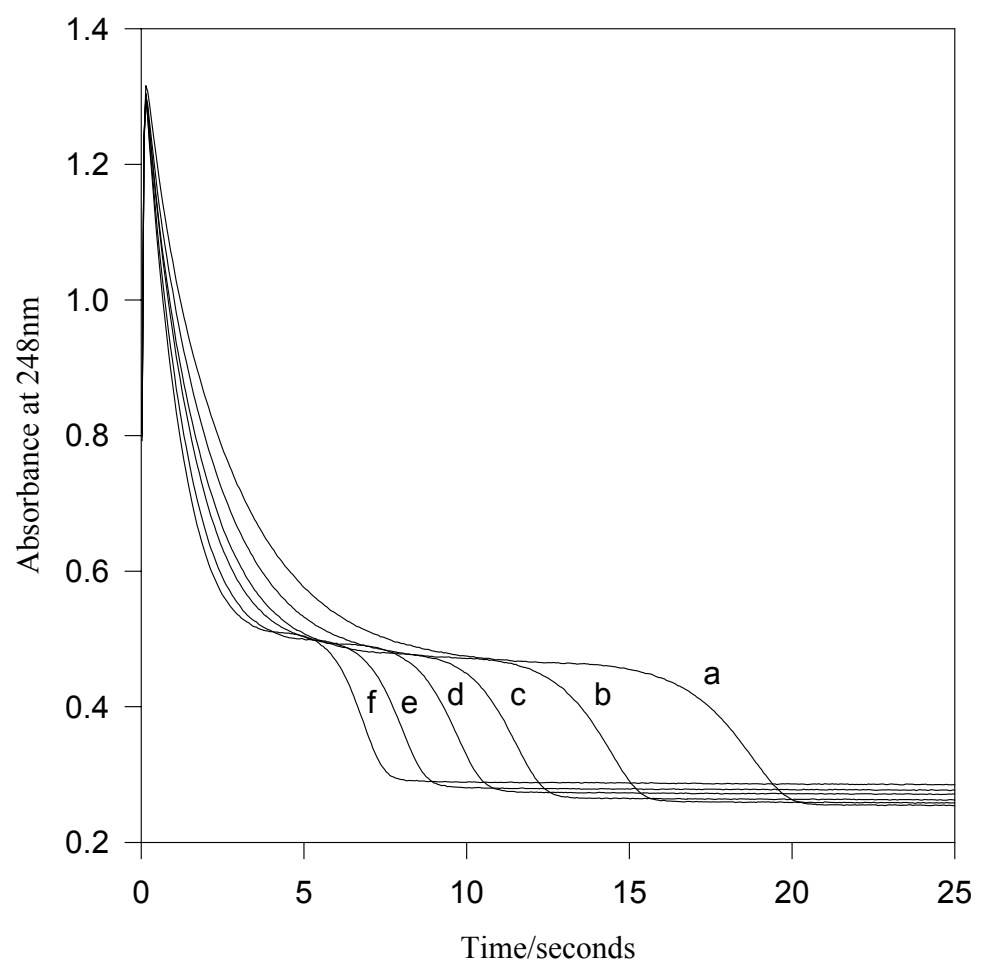

Figure 3.6a: Effect of chlorite on the depletion of TTTU in its oxidation by chlorite monitored at $248 \mathrm{~nm}$. The data displays two-stage kinetics. The products of the TTTU oxidation give residual absorbance as can be seen in the absorbance-time data. $[\mathrm{TTTU}]_{0}=7.5 \times 10^{-5} \mathrm{M},\left[\mathrm{HClO}_{4}\right]_{0}=0.125$ $\mathrm{M}, \mathrm{I}_{(\mathrm{NaClO} 4)}=1 \mathrm{M},\left[\mathrm{ClO}_{2}^{-}\right]_{0}=\left(\right.$ a) $5.0 \times 10^{-4} \mathrm{M}$, (b) $6.0 \times 10^{-4} \mathrm{M}$, (c) $7.0 \times 10^{-4} \mathrm{M}$, (d) $8.0 \times 10^{-4} \mathrm{M}$, (e) $9.0 \times 10^{-4} \mathrm{M}$, (f) $10.0 \times 10^{-4} \mathrm{M}$.

What might appear like a shutting down of the reaction about $5 \mathrm{~s}$ into the reaction is due to the formation of some TTTU intermediate, which will then either decompose or will be further oxidized to final products. This can be rationalized from the following analysis of our data: the final product, TMU, does not absorb at $248 \mathrm{~nm}$, and hence the observed 
absorbance at the end of the reaction is due to chlorine dioxide. The observed lack of change in absorbance at $248 \mathrm{~nm}(10 \mathrm{~s}$ into the reaction for trace a, figure $3.6 \mathrm{a})$ is partly derived from the fact that contribution from chlorine dioxide and depletion of TTTU effectively neutralize each other. Figure $3.6 \mathrm{~b}$ superimposes typical absorbance traces taken at $360 \mathrm{~nm}$ and $248 \mathrm{~nm}$. It shows that chlorine dioxide formation commences before then end of the first stage of the reaction and continues although at a slower rate after the end of this stage. It should be noted that chlorine dioxide production continues during the period when there is no activity in the absorbance at $248 \mathrm{~nm}$.

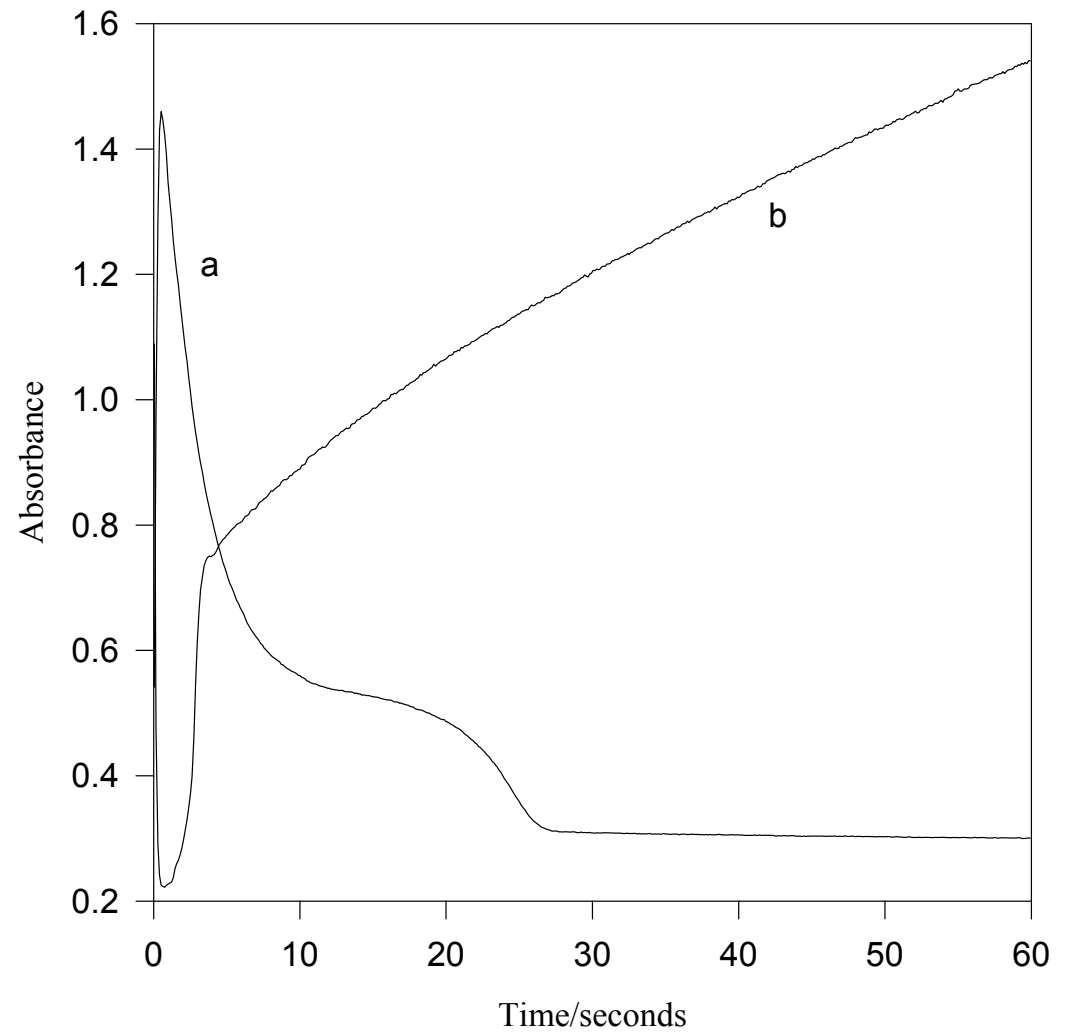

Figure 3.6b: Two traces superimposed at (a) $248 \mathrm{~nm}$ and (b) $360 \mathrm{~nm}$. $\left[\mathrm{ClO}_{2}{ }^{-}\right]_{0}=5.00 \times 10^{-4} \mathrm{M}$, $[\mathrm{TTTU}]_{0}=5.0 \times 10^{-5} \mathrm{M},\left[\mathrm{HClO}_{4}\right]_{0}=0.125 \mathrm{M}, \mathrm{I}_{(\mathrm{NaClO} 4)}=1 \mathrm{M}$. 


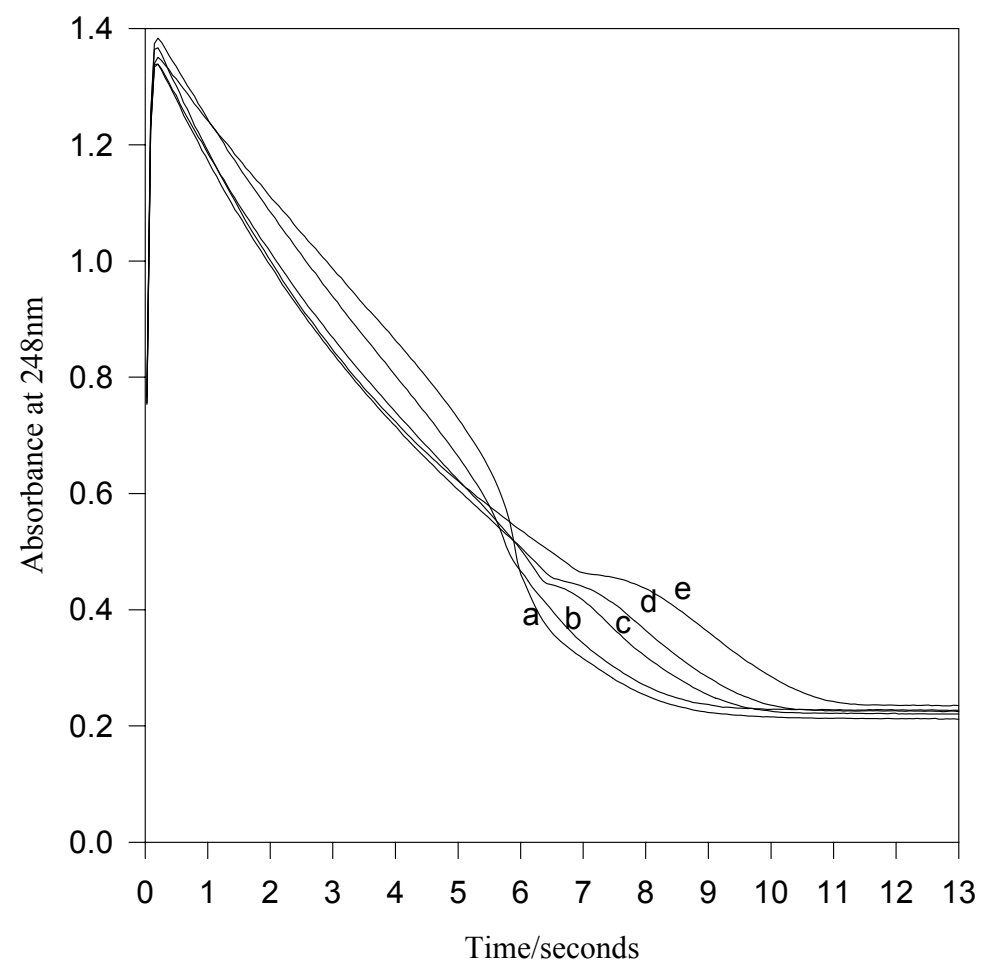

Figure 3.6c: Effect of acid on the depletion of TTTU in its oxidation by chlorite monitored at 248 $\mathrm{nm}$. The data also displays two-stage kinetics. [TTTU $]_{0}=7.5 \times 10^{-5} \mathrm{M},\left[\mathrm{ClO}_{2}{ }^{-}\right]_{0}=5.00 \times 10^{-4} \mathrm{M}$, $\mathrm{I}_{(\mathrm{NaClO} 4)}=1 \mathrm{M},\left[\mathrm{HClO}_{4}\right]_{0}=$ (a) $0.005 \mathrm{M}$, (b) $0.010 \mathrm{M}$, (c) $0.015 \mathrm{M}$, (d) $0.020 \mathrm{M}$, (e) $0.025 \mathrm{M}$.

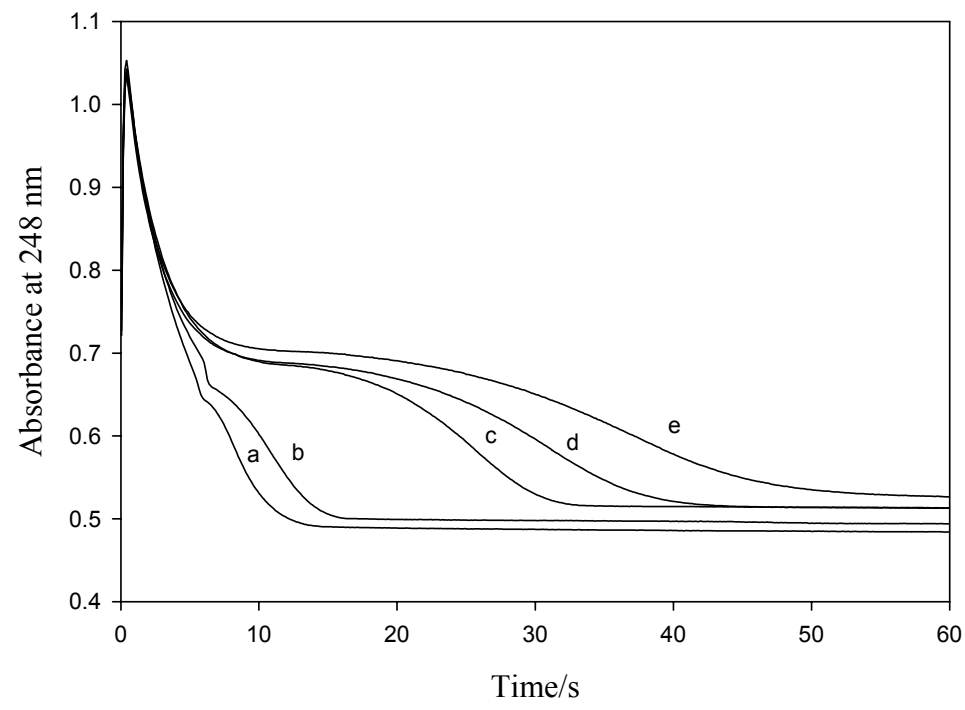

Figure 3.6d: Effect of acid on the depletion of TTTU in its oxidation by chlorite monitored at $248 \mathrm{~nm}$. The data still displays two-stage kinetics, with the rate of the first stage independent of acid concentrations while the second stage is catalyzed by lower acid concentrations. [TTTU $]_{0}=$ $5.00 \times 10^{-5} \mathrm{M},\left[\mathrm{ClO}_{2}^{-}\right]_{0}=5.00 \times 10^{-4} \mathrm{M}, \mathrm{I}_{(\mathrm{NaClO} 4)}=1 \mathrm{M},\left[\mathrm{HClO}_{4}\right]_{0}=$ (a) $0.025 \mathrm{M}$, (b) $0.075 \mathrm{M}$, (c) 0 . $1 \mathrm{M}$, (d) $0.15 \mathrm{M}$, (e) $0.2 \mathrm{M}$. 
Acid has opposite effects on the two stages of the reaction. Figure 3.6c shows a series of traces taken at low acid environments. Low acid environments deliver sigmoidal decay kinetics in the first stage, which give way to normal decay kinetics with a concave shape (see figure 3.6d) as the acid concentration is slowly increased. The second stage is very rapid at low acid concentrations, and in higher acid concentrations the intermediate lingers a bit longer. Pseudo-first order kinetics are never attained in conditions of high acid, coupled with high excess oxidant; $\mathrm{R} \geq 10$; and the rate of decay of TTTU in the first stage becomes insensitive to variations in acid, although the second phase is still inhibited by acid. The rate of TTTU depletion is first order (figures 3.7a and 3.7b) for all acid concentrations.

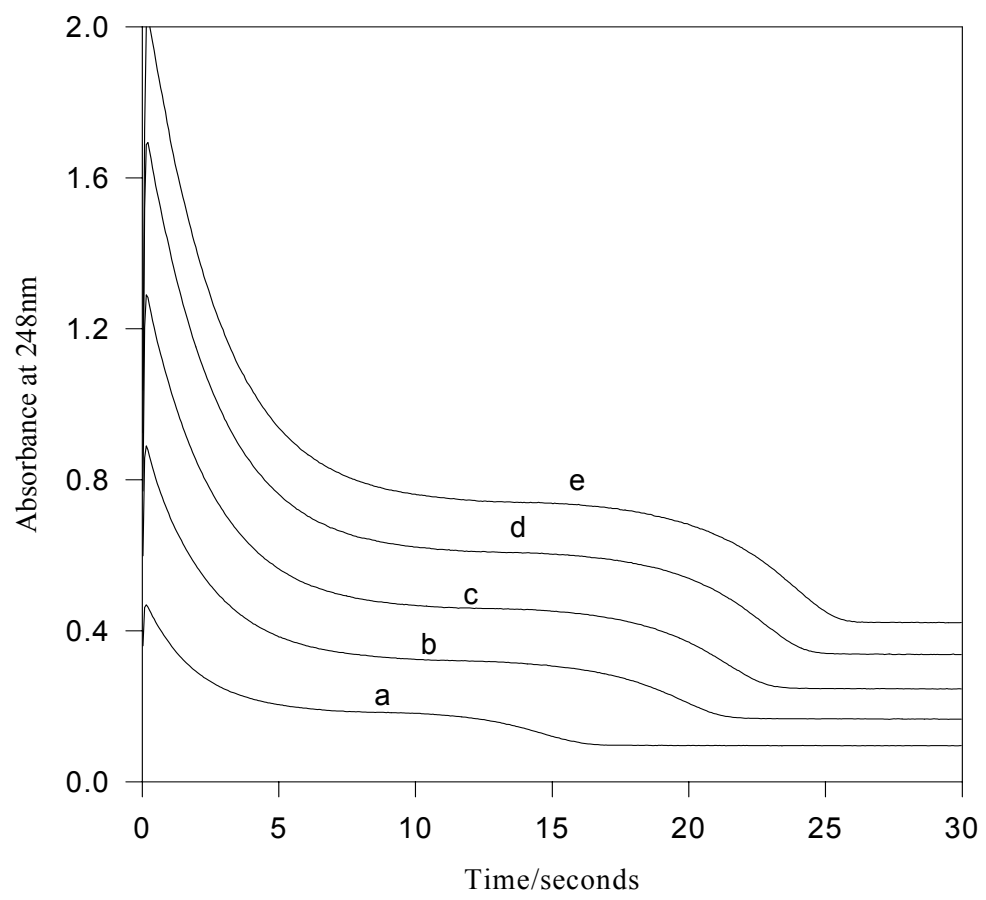

Figure 3.7a: Depletion of TTTU in its oxidation by chlorite. The data also displays two-stage kinetics. $\left[\mathrm{ClO}_{2}^{-}\right]_{0}=5.00 \times 10^{-4} \mathrm{M},\left[\mathrm{HClO}_{4}\right]_{0}=0.125 \mathrm{M},{ }_{(\mathrm{NaClO} 4)}=1 \mathrm{M},[\mathrm{TTTU}]_{0}=$ (a) $2.5 \times 10^{-5} \mathrm{M}$, (b) $5.0 \times 10^{-5} \mathrm{M}$, (c) $7.5 \times 10^{-5} \mathrm{M}$, (d) $10.0 \times 10^{-5} \mathrm{M}$, (e) $12.5 \times 10^{-5} \mathrm{M}$. 


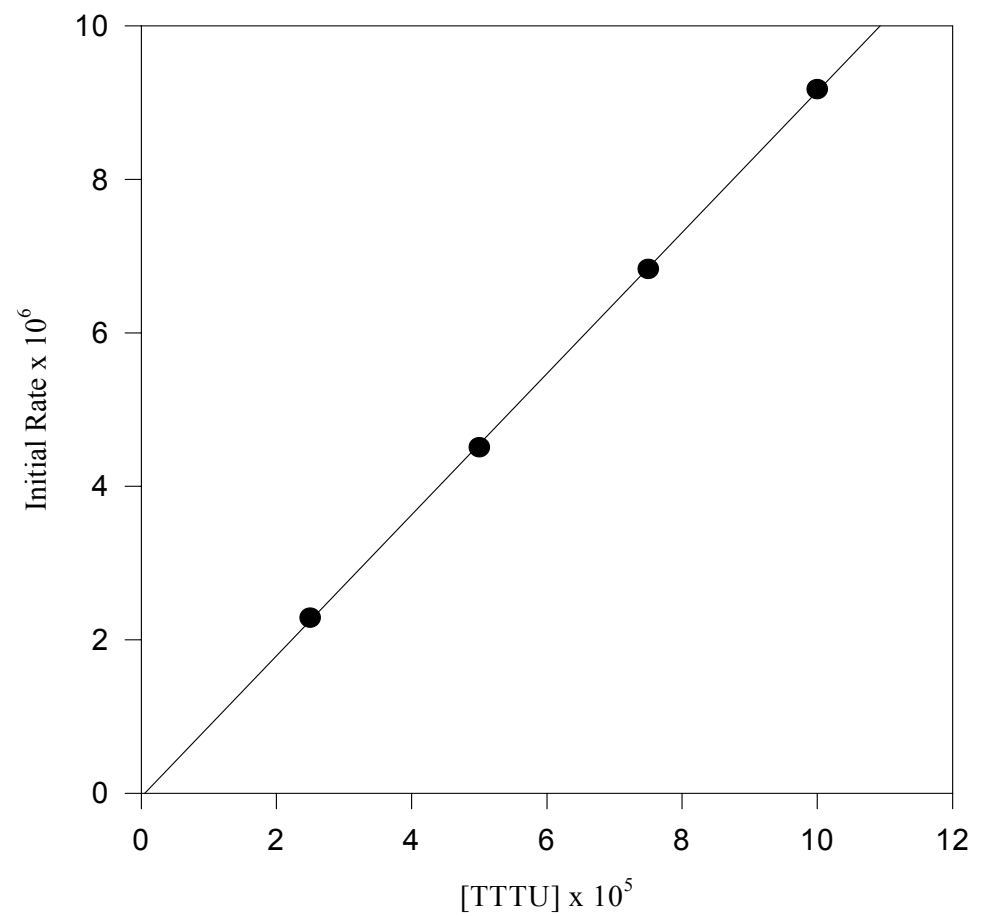

Figure 3.7b: Effect of initial TTTU concentration on the initial rate of consumption of TTTU. There is a solid linear dependence. $\left[\mathrm{ClO}_{2}^{-}\right]_{0}=5.00 \times 10^{-4} \mathrm{M},\left[\mathrm{HClO}_{4}\right]_{0}=0.125 \mathrm{M},\left(\mathrm{NaClO}_{4}\right)=1 \mathrm{M}$.

\subsubsection{Nature of intermediate species}

Figures 3.6a, 3.6b, 3.6d and 3.7a shows the formation of a long-lived intermediate species, about $10 \mathrm{~s}$ into the reaction, which rapidly decays after a short incubation period. This species appears to be more stable in acid and decomposes at a much slower rate in high acid environments. This intermediate species is the zwitterion sulfonic acid of TTTU, $\left(\mathrm{Me}_{2} \mathrm{~N}\right)_{2} \mathrm{CSO}_{3}$. Its further oxidation will give sulfate and urea as the final products. Its oxidation goes via the initial formation of bisulfite followed by its rapid oxidation to sulfate.

$$
\left(\mathrm{Me}_{2} \mathrm{~N}\right)_{2} \mathrm{CSO}_{3}+\mathrm{H}_{2} \mathrm{O} \rightleftharpoons\left(\mathrm{Me}_{2} \mathrm{~N}\right)_{2} \mathrm{C}=\mathrm{O}+\mathrm{HSO}_{3}^{-}+\mathrm{H}^{+}
$$


The sulfonic acid is the suggested intermediate based on the following experiment [9]: TTTU was mixed, in a $50 \%$ acetonitrile solution with exactly two equivalents of hydrogen peroxide. This solution was stirred, for over two hours at $-10{ }^{\circ} \mathrm{C}$. The resulting solution was allowed to thaw and stand overnight at room temperature. After about twelve hours some long, colorless, flat, triclinic-like crystals were formed. The supernatant solution also gave needle-like crystals after adding 50\% pet ether. Both sets of crystals, including the supernatant liquid, gave a spectrum with a peak at $224 \mathrm{~nm}$. The product of this synthesis was characterized as the (zwitterionic) sulfinic acid, $\left(\mathrm{Me}_{2} \mathrm{~N}\right)_{2} \mathrm{CSO}_{2}$ (through oxidation equivalents). The intermediate species formed in this reaction did not give the same spectrum as the sulfinic acid, and is assumed to be the sulfonic acid. The only other possibility, the sulfenic acid, is too unstable to be this longlived. Our previous work had shown that these organosulfur oxo-acids are more stable in acidic environments and quickly cleave the $\mathrm{C}-\mathrm{S}$ bond in basic environments [10-12] (e.g. guanidylation [13]). The basic environment allows the solvent, water, to act as a nucleophile, which can attack the positively charged four-electron, three-center carbon atom, eliminating the sulfur group, and forming the urea analogue of TTTU. High acid environments stabilize this zwitterion [11], and hence figures 3.6c and 3.6d show a slower decomposition of this intermediate species with increase in acid.

\subsubsection{Chlorine dioxide oxidations}

Most chlorine dioxide oxidations are retarded by acid, but figure 3.8 shows that acid catalyzes the oxidation of TTTU by chlorine dioxide at low acid concentrations. This is in agreement with experimental data in figure 3.4a done at the same low acid 
concentrations in which the rate of formation of chlorine dioxide increases with increase in acid concentrations and yet the effect is inhibitory at higher acid concentrations. However, some mild form of autocatalysis is also observed.

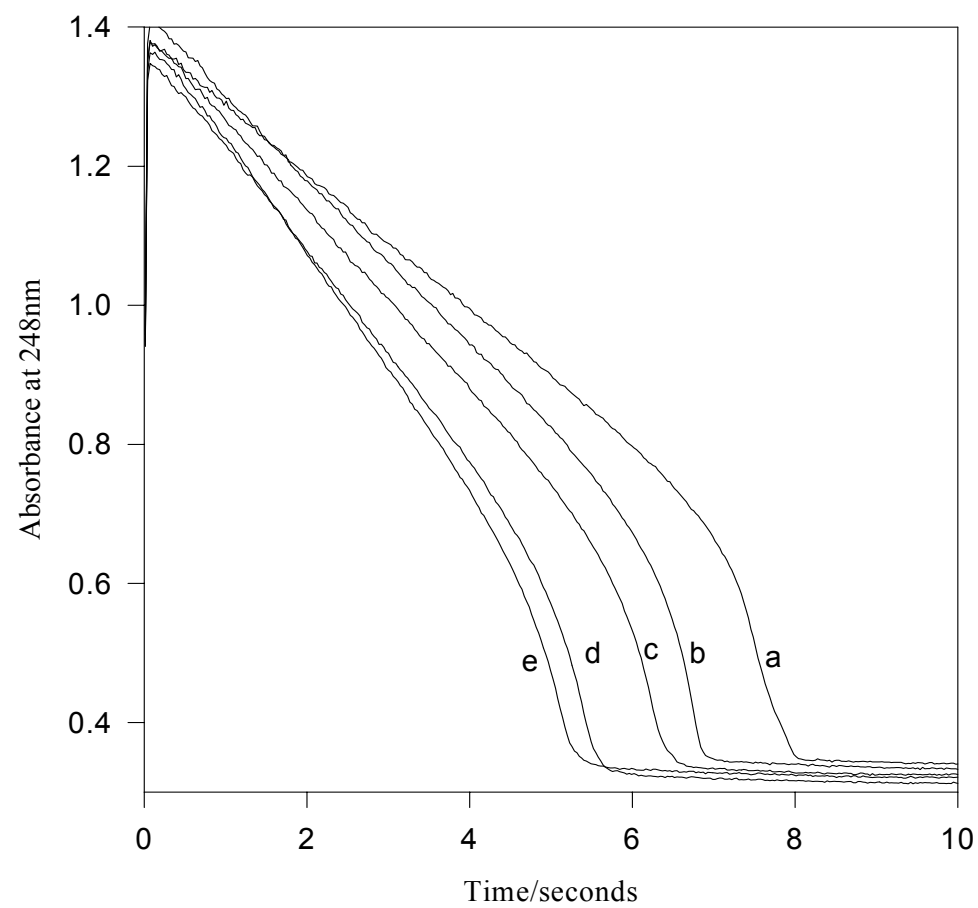

Figure 3.8: Effect of acid on the oxidation of TTTU by chlorine dioxide. Rate of depletion of TTTU increases with increase in acid concentrations. [TTTU $]_{0}=7.5 \times 10^{-5} \mathrm{M},\left[\mathrm{ClO}_{2}\right]_{0}=5.00 \mathrm{x}$ $10^{-4} \mathrm{M}, \mathrm{I}_{(\mathrm{NaClO} 4)}=1 \mathrm{M},\left[\mathrm{HClO}_{4}\right]_{0}=$ (a) $0.005 \mathrm{M}$, (b) $0.010 \mathrm{M}$, (c) $0.015 \mathrm{M}$, (d) $0.020 \mathrm{M}$, (e) 0.025 $\mathrm{M}$.

Figure 3.9a shows that the rate of depletion of TTTU in high acid and excess chlorine dioxide shows nearly zero order kinetics in TTTU. This is possible if there is a rapid and irreversible first step of the reaction that involves TTTU with the subsequent ratedetermining steps not involving TTTU. In figure $3.9 \mathrm{~b}$ the first $1 \mathrm{~s}$ of the reaction is expanded to show that initially there is a rapid and visible formation of an intermediate which is then slowly consumed to give products. In high acid, chlorine dioxide depletion follows nearly first order dependence (figure 3.10a). Data in figure 3.10a and 3.10b was 
performed in high excess of TTTU such that all chlorine dioxide is consumed at the end of the reaction.

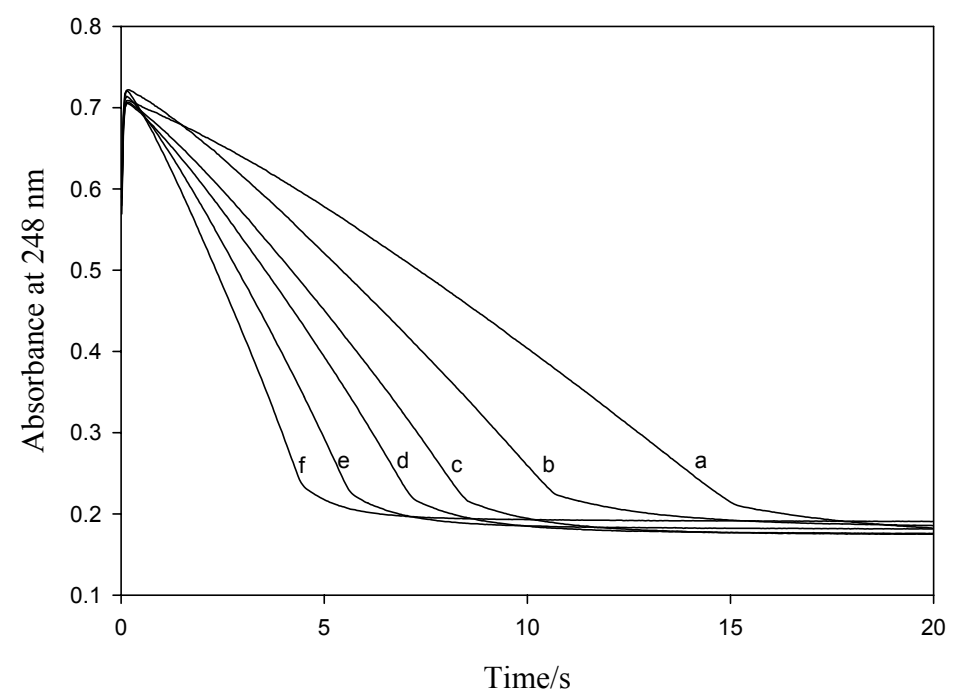

Figure 3.9a: Effect of chlorine dioxide in the depletion of TTTU. Rate of depletion of TTTU increases with increase in chlorine dioxide concentrations. $[\mathrm{TTTU}]_{0}=5.0 \times 10^{-5} \mathrm{M},\left[\mathrm{HClO}_{4}\right]_{0}=$ $0.125 \mathrm{M}, \mathrm{I}_{(\mathrm{NaClO} 4)}=1 \mathrm{M},\left[\mathrm{ClO}_{2}\right]_{0}=$ (a) $1.35 \times 10^{-4} \mathrm{M}$, (b) $1.80 \times 10^{-4} \mathrm{M}$, (c) $2.25 \times 10^{-4} \mathrm{M}$, (d) 2.70 x $10^{-4} \mathrm{M}$, (e) $3.60 \times 10^{-4} \mathrm{M}$, (f) $4.50 \times 10^{-4} \mathrm{M}$.

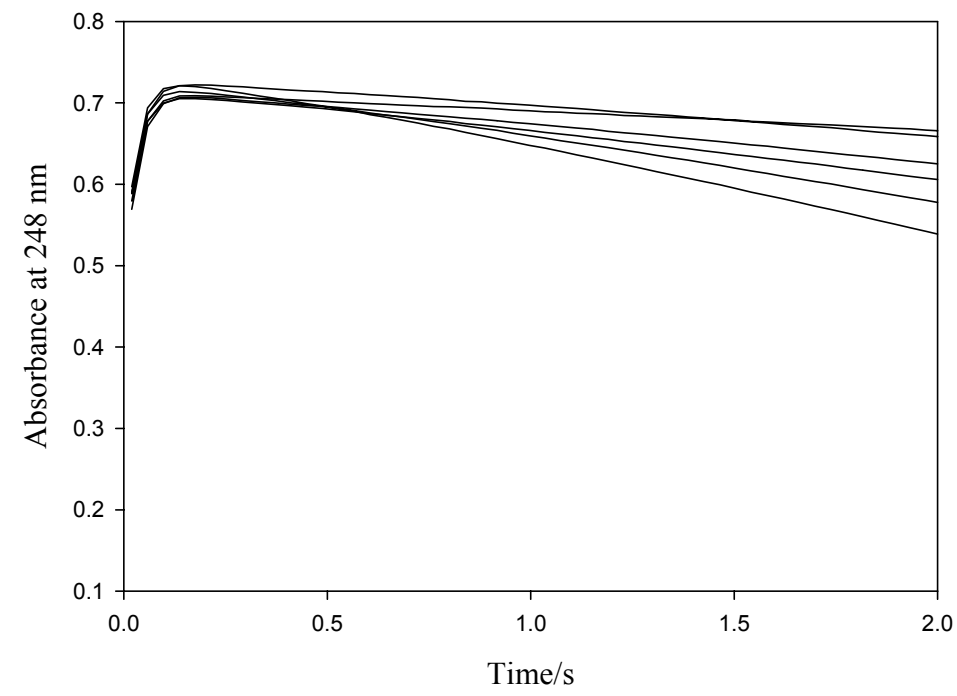

Figure 3.9b: Expansion of the first two seconds of the absorbance traces in figure 3.9a. There is an initial rapid formation of an adduct or intermediate within the first $0.5 \mathrm{~s}$. The rest of the reaction involves consumption of this adduct. 
The use of high acid simplifies the kinetics by using $\mathrm{HClO}_{2}$ as the oxidant with little or no contribution from $\mathrm{ClO}_{2}^{-}$.

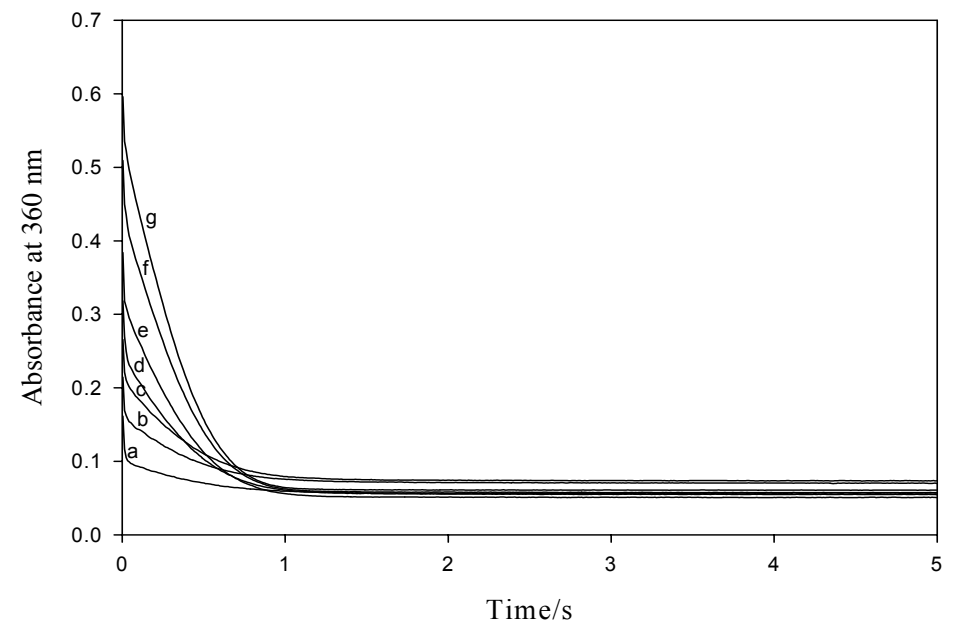

Figure 3.10a: Effect of chlorine dioxide on the oxidation of TTTU. The adduct observed in figures $3.9 \mathrm{a}$ and $3.9 \mathrm{~b}$ does not absorb at $360 \mathrm{~nm}$. The initial absorbencies observed are the ones expected from the contribution of chlorine dioxide on its own before its depletion commences.

Figure $3.10 \mathrm{~b}$ shows variation of TTTU while keeping chlorine dioxide concentration constant.

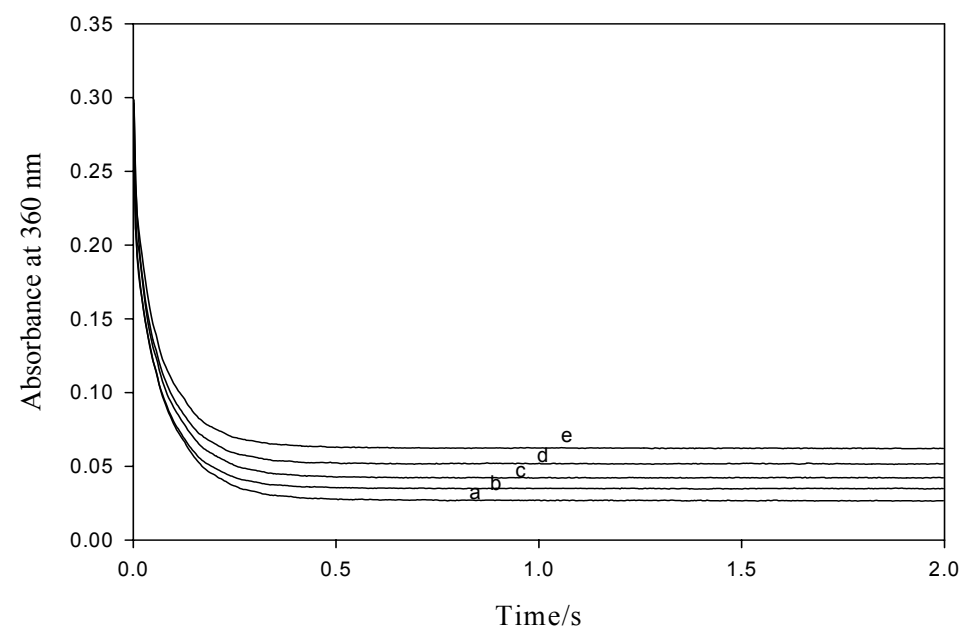

Figure 3.10b: Variation of TTTU in its oxidation by chlorine dioxide under close to pseudo-first oder kinetics environments. $\left[\mathrm{ClO}_{2}\right]_{0}=1.8 \times 10^{-4} \mathrm{M},\left[\mathrm{HClO}_{4}\right]_{0}=0.10 \mathrm{M}$, $[\mathrm{TTTU}]_{0}=$ (a) $2.50 \times 10^{-}$ ${ }^{4} \mathrm{M}$, (b) $3.75 \times 10^{-4} \mathrm{M}$, (c) $5.00 \times 10^{-4} \mathrm{M}$, (d) $6.25 \times 10^{-4} \mathrm{M}$, and (e) $=7.50 \times 10^{-4} \mathrm{M}$. 
Nearly pseudo-first order kinetics are observed, although the rate of chlorine dioxide consumption does not seem to be affected by TTTU. The observed differences in baselines are due to the varied input concentrations of TTTU and not to any changes in the kinetics. The surprising experimental finding was that chloride appears to have no effect on the reaction rate as shown in figure 3.11. It appeared to be more relevant in affecting the disproportionation of chlorite, which is the initial intermediate, formed as the reduction of chlorine dioxide commences.

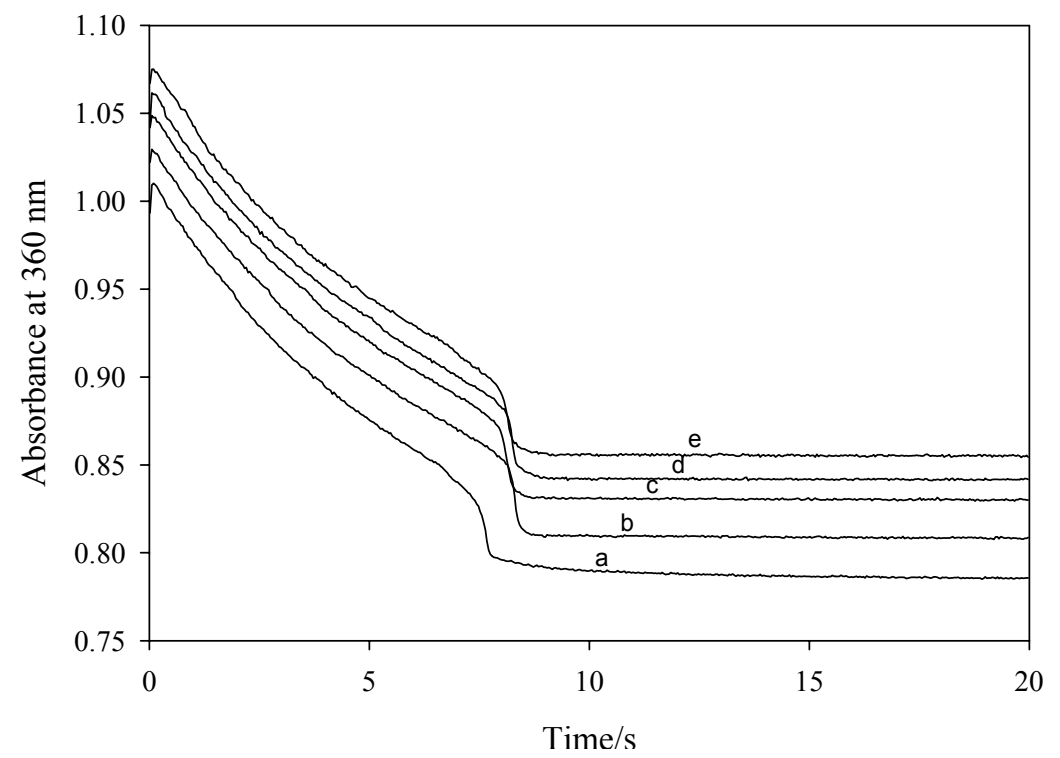

Figure 3.11: Effect of chloride on the oxidation of TTTU by chlorine dioxide. [TTTU] $]_{0}=2.5 \mathrm{x}$ $10^{-4} \mathrm{M},\left[\mathrm{ClO}_{2}\right]_{0}=1.25 \times 10^{-3} \mathrm{M},\left[\mathrm{HClO}_{4}\right]_{0}=0.005 \mathrm{M}, \mathrm{I}_{(\mathrm{NaClO})}=1 \mathrm{M},\left[\mathrm{Cl}^{-}\right]=$(a) $0.00 \mathrm{M}$, (b) $=$ $0.25 \mathrm{M},(\mathrm{c})=0.20 \mathrm{M},(\mathrm{d})=0.15 \mathrm{M},(\mathrm{e})=0.10 \mathrm{M}$.

\subsection{MECHANISM}

\subsubsection{Initial stages of the reaction}

The search for a plausible mechanism for this reaction should start with data in figure 3.6b. The short induction period with respect to absorptivity measurements at 360 $\mathrm{nm}$ comprises a very rapidly decreasing absorbance at $248 \mathrm{~nm}$. The next step would be 
the data in figure 3.6c in which the changes in consumption profiles of TTTU with acid concentrations have to be rationalized. Our mechanism should also be able to explain the apparent insensitivity of the reaction's induction period to acid concentrations at low acid concentrations (figure 3.4a) and the depressed chlorine dioxide formation with acid as observed in figure $3.4 \mathrm{~b}$ (high acid concentrations). Data in figure 3.6c suggests that there exists more than one oxidizing species in the reaction mixture and abundance of each species is dependent on the $\mathrm{pH}$ of the reaction medium. The $\mathrm{p} K_{\mathrm{a}}$ of chlorous acid is approximately $1.72[14]$ and hence in the $\mathrm{pH}$ ranges of $1-2$ one expects very dynamic changes in the relative concentrations of chlorous acid and chlorite anion. Thus, the change in observed kinetics with acid in this range can be attributed to the fact that both $\mathrm{HClO}_{2}$ and $\mathrm{ClO}_{2}^{-}$are active oxidants. We can set up a mass balance equation on the chlorine (III) species:

$$
[\mathrm{Cl}(\mathrm{III})]_{\mathrm{T}}=\left[\mathrm{ClO}_{2}^{-}\right]+\left[\mathrm{HClO}_{2}\right]
$$

Equation 3.4 relates the two reactive species; hence we can evaluate the following concentrations of $\mathrm{HClO}_{2}$ and $\mathrm{ClO}_{2}{ }^{-}$with respect to acid concentrations:

$$
\left[\mathrm{ClO}_{2}{ }^{-}\right]=\frac{[\mathrm{Cl}(\mathrm{III})]_{\mathrm{T}}}{1+K_{\mathrm{a}}\left[\mathrm{H}^{+}\right]} ;\left[\mathrm{HClO}_{2}\right]=\frac{K_{\mathrm{a}}^{-1}\left[\mathrm{H}^{+}\right][\mathrm{Cl}(\mathrm{III})]_{\mathrm{T}}}{1+K_{\mathrm{a}}{ }^{-1}\left[\mathrm{H}^{+}\right]}
$$

$\mathrm{HClO}_{2}$ concentrations dominate below $\mathrm{pH} 2$ and above this $\mathrm{pH}$ up to $\mathrm{pH} 4$ there will be a mixture of both the protonated and unprotonated forms of chlorine (III) species. Since the 
reaction is first order in chlorite and in TTTU (for the initial stages), we can write the rate of the reaction as:

$$
\text { Rate }=\frac{-\mathrm{d}[\mathrm{TTTU}]}{\mathrm{dt}}=[\mathrm{TTTU}]\left\{k_{1}\left[\mathrm{ClO}_{2}^{-}\right]+k_{2}\left[\mathrm{HClO}_{2}\right]\right\}
$$

Which simplifies to:

$$
\frac{-\mathrm{d}[\mathrm{TTTU}]}{\mathrm{dt}}=\frac{[\mathrm{TTTU}][\mathrm{Cl}(\mathrm{III})]_{\mathrm{T}}}{1+K_{\mathrm{a}}^{-1}\left[\mathrm{H}^{+}\right]}\left\{k_{1}+k_{2} K_{\mathrm{a}}^{-1}\left[\mathrm{H}^{+}\right]\right\}
$$

Equation 3.7 can be handled within certain concentration limits in which we can eliminate one of the terms. For example, in high acid conditions, $\left[\mathrm{ClO}_{2}{ }^{-}\right] \approx 0$ and $\left[\mathrm{HClO}_{2}\right]$ $\approx[\mathrm{Cl}(\mathrm{III})]_{\mathrm{T}}$; this simplifies to only the second term in equation 3.6:

$$
\text { Rate }=k_{2}[\mathrm{Cl}(\mathrm{III})]_{0}[\mathrm{TTTU}]
$$

We can then calculate a limiting $k_{2}$ value in high acid concentrations (e.g. figure 3.6d). By using $\mathrm{p} K_{\mathrm{a}}=1.72$ [14], and using known values for initial reagent concentrations, initial rate equation 3.7 can thus be calculated at each data point and a value for $k_{1}$ is evaluated. A series of experiments were then run at very high oxidant to reductant ratios and high acid concentrations such that $\left[\mathrm{H}^{+}\right]_{0},\left[\mathrm{ClO}_{2}\right]_{0}>>[\mathrm{TTTU}]$ in search for pseudofirst order kinetics, as shown in figure 3.10b. Or rate of reaction then becomes: 


$$
\frac{-\mathrm{d}[\mathrm{TTTU}]}{\mathrm{dt}}=k^{\mathrm{obs}}[\mathrm{TTTU}] ; \quad k^{\mathrm{obs}}=k_{2}\left[\mathrm{HClO}_{2}\right]=\mathrm{constant}
$$

This showed that the order of the reaction with respect to $\left[\mathrm{HClO}_{2}\right]$ is unity.

\subsubsection{Chlorine dioxide formation}

The initial stages of the reaction involve the formation of the reactive oxychlorine species, $\mathrm{HOCl}$. $\mathrm{HOCl}$ can then either further oxidize the substrate or react with chlorite to form chlorine dioxide. The first metabolite to be formed is a very unstable zwitterionic sulfenic acid species which should then be quickly further oxidized to the sulfinic acid zwitterions:

$$
\begin{aligned}
& \mathrm{ClO}_{2}^{-}+\left(\mathrm{Me}_{2} \mathrm{~N}\right)_{2} \mathrm{C}=\mathrm{S}+\mathrm{H}^{+} \rightarrow\left[\left(\mathrm{Me}_{2} \mathrm{~N}\right)_{2} \mathrm{C}-\mathrm{S}-\mathrm{O}\right]+\mathrm{HOCl} \\
& \mathrm{ClO}_{2}^{-}+\left[\left(\mathrm{Me}_{2} \mathrm{~N}\right)_{2} \mathrm{C}-\mathrm{S}-\mathrm{O}\right]+\mathrm{H}^{+} \rightarrow\left[\left(\mathrm{Me}_{2} \mathrm{~N}\right)_{2} \mathrm{C}-\mathrm{S}-\mathrm{O}_{2}\right]+\mathrm{HOCl} \\
& \mathrm{ClO}_{2}^{-}+\mathrm{HOCl}+\mathrm{H}^{+} \rightleftharpoons \mathrm{Cl}_{2} \mathrm{O}_{2}+\mathrm{H}_{2} \mathrm{O} \\
& \mathrm{ClO}_{2}^{-}+\mathrm{Cl}_{2} \mathrm{O}_{2} \rightarrow 2 \mathrm{ClO}_{2(\text { aq })}+\mathrm{Cl}^{-}
\end{aligned}
$$

Since reaction 3.12 is considered to be a fast reaction, the rate of formation of chlorine dioxide becomes dependent upon the rate of formation of $\mathrm{HOCl}$, from the oxidation of TTTU. This reaction sequence, $3.10-3.13$ is sufficient to explain data in figure 3.5 , in which the rate of formation of chlorine dioxide increased with increasing initial TTTU concentrations. The observed complex acid dependence can be explained via equation 3.7. In low acid concentrations, one can approximate the denominator to unity, and one would observe a mild catalysis based on the numerator term in acid. As acid 
concentration is increased, equation 3.7 predicts a saturation in which acid will no longer have an effect. Further retardation observed with increase in acid will be derived from the protonation of the TTTU, which occurs at much higher acid concentrations than protonation of chlorous acid.

$$
\left(\mathrm{Me}_{2} \mathrm{~N}\right)_{2} \mathrm{C}=\mathrm{S}+\mathrm{H}^{+} \rightleftharpoons\left[\left(\mathrm{Me}_{2} \mathrm{~N}\right)_{2} \mathrm{C}=\mathrm{S}-\mathrm{H}\right]^{+} ; K_{\mathrm{b}}
$$

If the protonated species is inert to attack by $\mathrm{HClO}_{2}$, then equation 3.7 can be re-written as:

$$
\frac{-\mathrm{d}[\mathrm{TTTU}]}{\mathrm{dt}}=\frac{[\mathrm{TTTU}][\mathrm{Cl}(\mathrm{III})]_{\mathrm{T}}}{\left(1+K_{\mathrm{a}}^{-1}\left[\mathrm{H}^{+}\right]\right)\left(1+K_{\mathrm{b}}{ }^{-1}\left[\mathrm{H}^{+}\right]\right)}\left\{k_{1}+k_{2} K_{\mathrm{a}}^{-1}\left[\mathrm{H}^{+}\right]\right\}
$$

to account for the retardation due to the formation of protonated TTTU. Equation 3.15 can then account for the mild catalysis at low acid, no effect at intermediate acid concentrations, and the retardation observed at high acid concentrations. The rate of formation of chlorine dioxide will mimic the rate of formation of $\mathrm{HOCl}$ which is controlled by equation 3.15 .

\subsubsection{Consumption of TTTU}

Figures $3.6 \mathrm{a}-3.6 \mathrm{~d}$, and figure 3.8 , show that the consumption of TTTU occurs in two distinct stages. The first stage gives way to a slow phase followed by a rapid decay to 
form products. The expected decay route for TTTU should involve the following pathway:
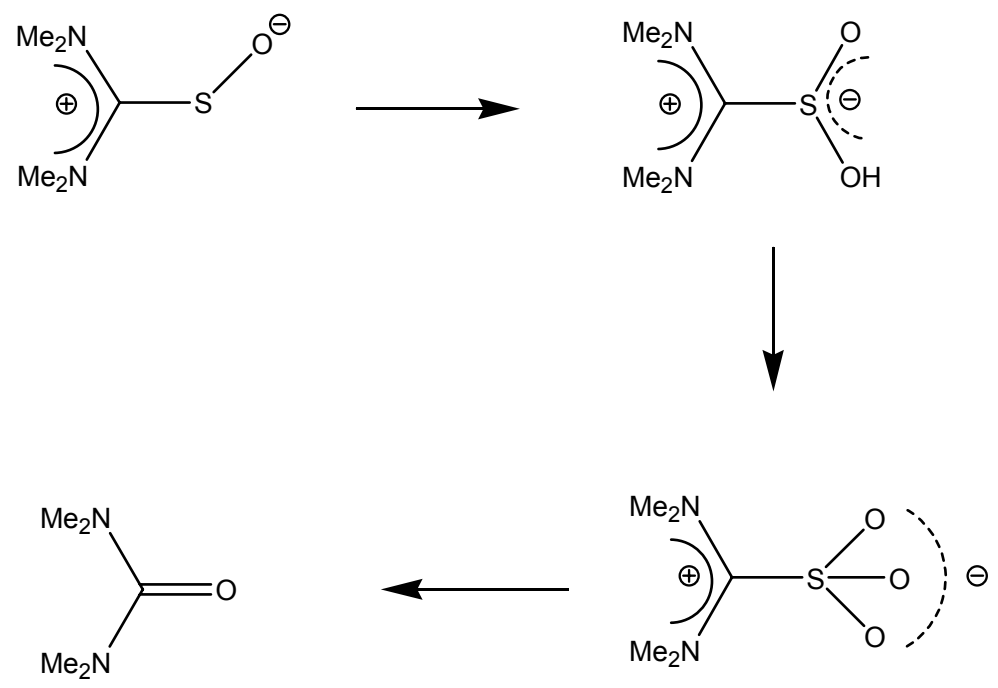

The second stage of the reaction involves the cleavage of the $\mathrm{C}-\mathrm{S}$ bond to yield tetramethylurea, TMU, and sulfate. By synthesizing the sulfinic zwitterion (see section 3.2.1.5), we have managed to prove that the last step involves the oxidation of the sulfonic acid to give urea and sulfate. This is an irreversible entropy-driven process in which the sulfonic acid breaks up. The rate of reaction of this process is determined by the rate of decomposition of the sulfonic acid, since we anticipate that the oxidation of bisulfate to sulfate to be fast.

\subsubsection{Chlorine dioxide consumption}

The main route for chlorine dioxide consumption is via its direct reaction with TTTU. Chlorine dioxide should react very slowly or should be inert to the zwitterion sulfur oxo-acids. Further oxidation of these oxo-acids will be accomplished through chlorite and $\mathrm{HOCl}$. The first step in the reaction of chlorine dioxide with TTTU involves 
the rapid formation of an intimate adduct of the two species, which is free energy driven. The sulfur center is nucleophilic with two lone pairs of electrons, while the chlorine center has an unpaired electron. The formation of the adduct, $\left[\left(\mathrm{Me}_{2} \mathrm{~N}\right)_{2} \mathrm{CSClO}_{2}\right]$, should be instantaneous:

$$
\left(\mathrm{Me}_{2} \mathrm{~N}\right)_{2} \mathrm{C}=\mathrm{S}+\mathrm{ClO}_{2} \rightarrow\left[\left(\mathrm{Me}_{2} \mathrm{~N}\right)_{2} \mathrm{CSClO}_{2}\right]
$$
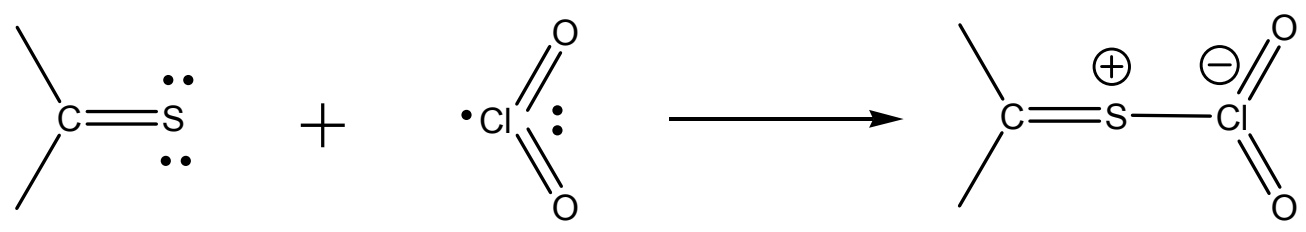

Our experimental data in figure $3.9 \mathrm{~b}$ shows that this adduct is formed within milliseconds of mixing of the two reagents. This adduct formation has been suggested in other chlorine dioxide oxidations [15-17]. Further reaction of the adduct will involve another chlorine dioxide molecule coupled with hydrolysis to yield the sulfenic acid zwitterion in a rate determining step:

$$
\left[\left(\mathrm{Me}_{2} \mathrm{~N}\right)_{2} \mathrm{CSClO}_{2}\right]+\mathrm{ClO}_{2}+\mathrm{H}_{2} \mathrm{O} \rightarrow\left[\left(\mathrm{Me}_{2} \mathrm{~N}\right)_{2} \mathrm{C}-\mathrm{S}-\mathrm{O}\right]+2 \mathrm{ClO}_{2}^{-}+2 \mathrm{H}^{+}
$$

The product of reaction 3.17 could either be chlorite or chlorous acid depending on the $\mathrm{pH}$ of the reaction solution. Chlorite is a more labile and active oxidizing agent than chlorine dioxide. Reactions 3.16 and 3.17 show that one TTTU molecule produces two molecules of chlorite. If chlorite oxidations are faster than chlorine dioxide's, we expect autocatalysis. This is indeed the case as shown by the data in figures 3.6c and 3.8. High concentrations of acid should destroy this autocatalysis as the reaction will predominantly 
proceed through chlorous acid. Data obtained in high acid concentrations as in figures 3.6a, 3.6b, 3.10a and 3.10b does not show any hint of sigmoidal decay kinetics which is indicative of autocatalysis.

\subsubsection{Overall reaction mechanism and computer modeling}

The modeling of the overall reaction scheme was done in stages, with the easiest system modeled first: the chlorine dioxide/TTTU reaction in high acid (figures 3.10a and 3.10b). The degree of complexity was then extended to low acid conditions where autocatalysis prevailed (figure 3.8). The overall reaction scheme involving chlorite was then performed last after satisfying the two simpler systems, and utilizing some of the kinetic constants optimized in the previous simulations.

Table 3.1: Chlorine dioxide/TTTU reactions in high acid

\begin{tabular}{lll}
\hline & Reaction & $k_{\mathrm{f}} ; k_{\mathrm{r}}$ \\
\hline $\mathrm{M} 1$ & $\mathrm{ClO}_{2}+\mathrm{R}_{2} \mathrm{C}=\mathrm{S} \rightarrow\left[\mathrm{R}_{2} \mathrm{CSClO}_{2}\right]$ & $2.5 \times 10^{3}$ \\
$\mathrm{M} 2$ & {$\left[\mathrm{R}_{2} \mathrm{CSClO}_{2}\right]+\mathrm{ClO}_{2}+\mathrm{H}_{2} \mathrm{O} \rightarrow \mathrm{R}_{2} \mathrm{C}-\mathrm{SO}+2 \mathrm{HClO}_{2}$} & 15 \\
$\mathrm{M} 3$ & $\mathrm{R}_{2} \mathrm{C}=\mathrm{S}+\mathrm{HClO}_{2} \rightarrow \mathrm{R}_{2} \mathrm{C}-\mathrm{SO}+\mathrm{HOCl}$ & 75 \\
$\mathrm{M} 4$ & $\mathrm{R}_{2} \mathrm{C}-\mathrm{SO}+\mathrm{HClO}_{2} \rightarrow \mathrm{R}_{2} \mathrm{C}-\mathrm{SO}_{2}+\mathrm{HOCl}$ & 500 \\
M5 & $\mathrm{R}_{2} \mathrm{C}-\mathrm{SO}_{2}+\mathrm{HClO}_{2} \rightarrow \mathrm{R}_{2}{\mathrm{C}-\mathrm{SO}_{3}+\mathrm{HOCl}}_{2}$ & $1 \times 10^{3}$ \\
M6 & $\mathrm{R}_{2} \mathrm{C}-\mathrm{SO}+\mathrm{HOCl} \rightarrow \mathrm{R}_{2} \mathrm{C}-\mathrm{SO}_{2}+\mathrm{H}^{+}+\mathrm{Cl}^{-}$ & $5 \times 10^{3}$ \\
M7 & $\mathrm{R}_{2} \mathrm{C}-\mathrm{SO}_{2}+\mathrm{HOCl} \rightarrow \mathrm{R}_{2} \mathrm{C}-\mathrm{SO}_{3}+\mathrm{H}^{+}+\mathrm{Cl}^{-}$ & $5 \times 10^{2}$ \\
M8 & $\mathrm{R}_{2} \mathrm{C}-\mathrm{SO}_{3}+\mathrm{HOCl} \rightarrow \mathrm{R}_{2} \mathrm{C}=\mathrm{O}+\mathrm{SO}_{4}{ }^{2-}+3 \mathrm{H}^{+}+\mathrm{Cl}^{-}$ & $5 \times 10^{3}$ \\
M9 & $\mathrm{R}_{2} \mathrm{C}-\mathrm{SO}_{3}+\mathrm{H}_{2} \mathrm{O} \rightarrow \mathrm{R}_{2} \mathrm{C}=\mathrm{O}+\mathrm{HSO}_{3}{ }^{-}+\mathrm{H}^{+}$ & 100 \\
M10 & $\mathrm{HSO}_{3}{ }^{-}+\mathrm{HOCl} \rightarrow \mathrm{SO}_{4}{ }^{2-}+2 \mathrm{H}^{+}+\mathrm{Cl}^{-}$ & $5 \times 10^{5}$ \\
\hline
\end{tabular}

The mechanism adopted for the simple chlorine dioxide/TTTU reaction is shown in Table 3.1. Its simplicity lies in the fact that it only has two adjustable kinetic parameters, $k_{\mathrm{M} 2}$ 
and $k_{\mathrm{M} 8}$. The assumed fast first reaction relinquished control of kinetics to the second reaction. Once the first S-oxide, sulfenic acid was formed, subsequent oxidations were not considered rate-determining. Reaction M9 is fast, and so the hydrolysis rate and the equilibrium constant for reaction M8 became important. Since no autocatalysis is observed under these conditions, evoking the $\mathrm{Cl}_{2} \mathrm{O}_{2}$ intermediate was unnecessary.

\subsection{CONCLUSION}

The oxidation kinetics of TTTU by chlorite is more complex and faster when compared to the oxidation of the unsubstituted thiourea [18]. TTTU reactions were also found to be faster than comparable oxidations of Trimethylthiourea (TMTU) [19]. Oxidation of TTTU by chlorite is complicated by the formation of a stable intermediate: the sulfonic acid which exists predominantly in the zwitterionic form. The rate of oxidation of the sulfonic acid was determined by its rate of decomposition, which is inhibited by acid. The oxidation of TTTU by chlorine dioxide was autocatalytic and inhibited by acid at high acid concentrations. Acid catalyzed the chlorine dioxide oxidations at low acid concentrations. The initial step in the chlorine dioxide oxidations involved the formation of an adduct of the radical chlorine dioxide species with the electron-rich sulfur center of the thiocarbamide followed by reaction of the adduct with another chlorine dioxide molecule and subsequent hydrolysis to yield chlorite and the sulfenic acid zwitterion. 


\section{References}

1. S. Teramoto, M. Kaneda, H. Aoyama, and Y. Shirasu, "Correlation between the molecular structure of $\mathrm{N}$-alkylureas and $\mathrm{N}$-alkylthioureas and their teratogenic properties," Teratology 23, 335-342 (1981).

2. E. F. Stula, H. Sherman, and J. R. Barnes, "Thyroid tumors in rats from tetramethylthiourea," J. Environ. Pathol. Toxicol. 2, 889-906 (1979).

3. C. Raby, J. F. Lagorce, A. C. Jambut-Absil, J. Buxeraud, and G. Catanzano, "The mechanism of action of synthetic antithyroid drugs: Iodine complexation during oxidation of iodide," Endocrinology 126, 1683-1691 (1990).

4. J. Dai, C. Xie, R. Vincent, and A. Churg, "Air pollution particles produce airway wall remodeling in rat tracheal explants," Am. J. Respir. Cell Mol. Biol. 29, 352358 (2003).

5. J. Dai, C. Xie, and A. Churg, "Iron loading makes a nonfibrogenic model air pollutant particle fibrogenic in rat tracheal explants," Am. J. Respir. Cell Mol. Biol. 26, 685-693 (2002).

6. P. C. Singhal, S. Gupta, P. Sharma, H. Shah, N. Shah, and P. Patel, "Receptor mediated endocytosis by mesangial cells modulates transmigration of macrophages," Inflammation 24, 519-532 (2000).

7. K. E. Driscoll, J. M. Carter, B. W. Howard, D. Hassenbein, Y. M. W. Janssen, and B. T. Mossman, "Crocidolite activates NF-kB and MIP-2 gene expression in rat alveolar epithelial cells. Role of mitochondrial-derived oxidants," Environ. Health Perspect. Suppl. 106, 1171-1174 (1998).

8. A. K. Horvath, I. Nagypal, G. Peintler, I. R. Epstein, and K. Kustin, "Kinetics and mechanism of the decomposition of chlorous acid," J. Phys. Chem. A 107, 69666973 (2003).

9. A. E. Miller, J. J. Bischoff, and K. Pae, "Chemistry of aminoiminomethanesulfinic and -sulfonic acids related to the toxicity of thioureas," Chem. Res. Toxicol. 1, 169174 (1988).

10. S. V. Makarov, C. Mundoma, J. H. Penn, J. L. Petersen, S. A. Svarovsky, and R. H. Simoyi, "Structure and stability of aminoiminomethanesulfonic acid," Inorg. Chim. Acta 286, 149-154 (1999).

11. S. V. Makarov, C. Mundoma, J. H. Penn, S. A. Svarovsky, and R. H. Simoyi, "New and surprising experimental results from the oxidation of sulfinic and sulfonic acids," J. Phys. Chem. A 102, 6786-6792 (1998). 
12. J. F. Ojo, A. Otoikhian, R. Olojo, and R. H. Simoyi, "Oxyhalogen-sulfur chemistry: Nonlinear oxidation kinetics of hydroxymethanesulfinic acid by acidic iodate," J. Phys. Chem. A 108, 2457-2463 (2004).

13. C. A. Maryanoff, R. C. Stanzione, J. N. Plampin, and J. E. Mills, "A convenient synthesis of guanidines from thioureas," Journal of Organic Chemistry 51, 18821884 (1986).

14. E. Hwang, J. N. Cash, and M. J. Zabik, "Chlorine and chlorine dioxide treatment to reduce or remove EBDCs and ETU residues in a solution," J. Agricult. Food Chem. 50, 4734-4742 (2002).

15. J. Darkwa, R. Olojo, E. Chikwana, and R. H. Simoyi, "Antioxidant chemistry: oxidation of L-cysteine and its metabolites by chlorite and chlorine dioxide," J. Phys. Chem. A 108, 5576-5587 (2004).

16. A. K. Horvath, I. Nagypal, and I. R. Epstein, "Kinetics and mechanism of the chlorine dioxide-tetrathionate reaction," J. Phys. Chem. A 107, 10063-10068 (2003).

17. A. K. Horvath and I. Nagypal, "Kinetics and mechanism of the reaction between thiosulfate and chlorine dioxide," J. Phys. Chem. A 102, 7267-7272 (1998).

18. I. R. Epstein, K. Kustin, and R. H. Simoyi, "Systematic design of chemical oscillators. 78. Kinetics and mechanism of the chlorite-thiourea reaction in acidic medium," J. Phys. Chem. 96, 5852-5856 (1992).

19. T. R. Chigwada and R. H. Simoyi, "S-oxygenation of thiocarbamides II: Oxidation of trimethylthiourea by chlorite and chlorine dioxide," J. Phys. Chem. A 109, 10941104 (2005). 


\section{CHAPTER 4}

\section{Oxidation of Trimethylthiourea by Chlorite and Chlorine Dioxide}

\subsection{INTRODUCTION}

Sulfur chemistry is known to generate a wide range of nonlinear behavior that surpasses that generated by oxyhalogen chemistry [1]. All the exotic dynamics observed in oxyhalogen chemistry can be observed in sulfur chemistry, e.g. chemical oscillations [2], clock reactions [3], traveling waves [4], spatiotemporal pattern formation [5], oligooscillations [6], and chemical chaos [7,8]. For example, the chlorite-thiourea reaction was reported to sustain a traveling wave of chlorine dioxide in neutral to acidic environments [4,9-11]. The oxidation of sulfur compounds, such as thiocyanate [12,13], thiourea [14-16], and methylthiourea [17] often produces nonlinear dynamical behavior. The origin of this observed nonlinear behavior is not well understood since not much is known about the kinetics and the mechanisms of sulfur reactions. Complete understanding of the nonlinear dynamical behavior is only possible if the mechanisms of these reactions, especially the oxidation of sulfur compounds, are known in detail.

Trimethylthiourea, TMTU, a member of the group of compounds called thiocarbamides was reported to be a thyroid gland carcinogen in rats $[18,19]$. It has also been reported to cause skin and eye irritations, and is used in the rubber industry as a vulcanization accelerator. While the biological activities of thiourea and dimethylthiourea are well documented, not much has been mentioned in literature with regards to the toxicity of TMTU, and the origin of its carcinogenicity. Generally, the oxidation of 
thioureas generates genotoxic products, and thus evaluation of the oxidation mechanisms of thioureas could have a predictive advantage in terms of their toxicities. An attempt to generalize reaction systems of sulfur compounds (thiocarbamides especially) failed, because each system seems to present a unique reactivity; hence each system has to be studied independently.

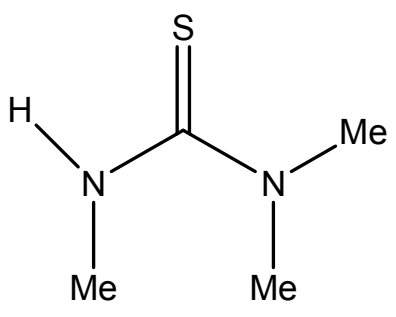

Trimethylthiourea

In this chapter we report on the detailed study of oxidation of TMTU by acidic chlorite. Our interest in this compound is to enable us to better understand the origin and mechanism of toxicity leading to thyroid tumors in rats. Previous studies have reported on the vastly-different spatial patterns obtained in chlorite/TMTU and chlorite/tetramethylthiourea systems [5]. While both systems appear to be bistable and autocatalytic, they differ greatly in the structures they generate in unstirred conditions. The conclusion had been that the observed symmetry-breaking bifurcations were heavily dependent on the chemical kinetics and energetics of the oxidation reactions, although no attempt had been made to interpret these results in terms of reaction mechanisms. This study can give insights to the origin of such disparities. 


\subsection{RESULTS}

\subsubsection{Stoichiometry}

In excess chlorite conditions, the stoichiometry of the reaction was determined to be an oxidant to reductant ratio, $(\mathrm{R})$, of 2:1:

$2 \mathrm{ClO}_{2}{ }^{-}+\mathrm{Me}_{2} \mathrm{~N}(\mathrm{NHMe}) \mathrm{C}=\mathrm{S}+\mathrm{H}_{2} \mathrm{O} \rightarrow 2 \mathrm{Cl}^{-}+\mathrm{Me}_{2} \mathrm{~N}(\mathrm{NHMe}) \mathrm{C}=\mathrm{O}+\mathrm{SO}_{4}{ }^{2-}+2 \mathrm{H}^{+}$

This stoichiometry was determined by a combination of spectrophotometric, titrimetric, and gravimetric techniques.

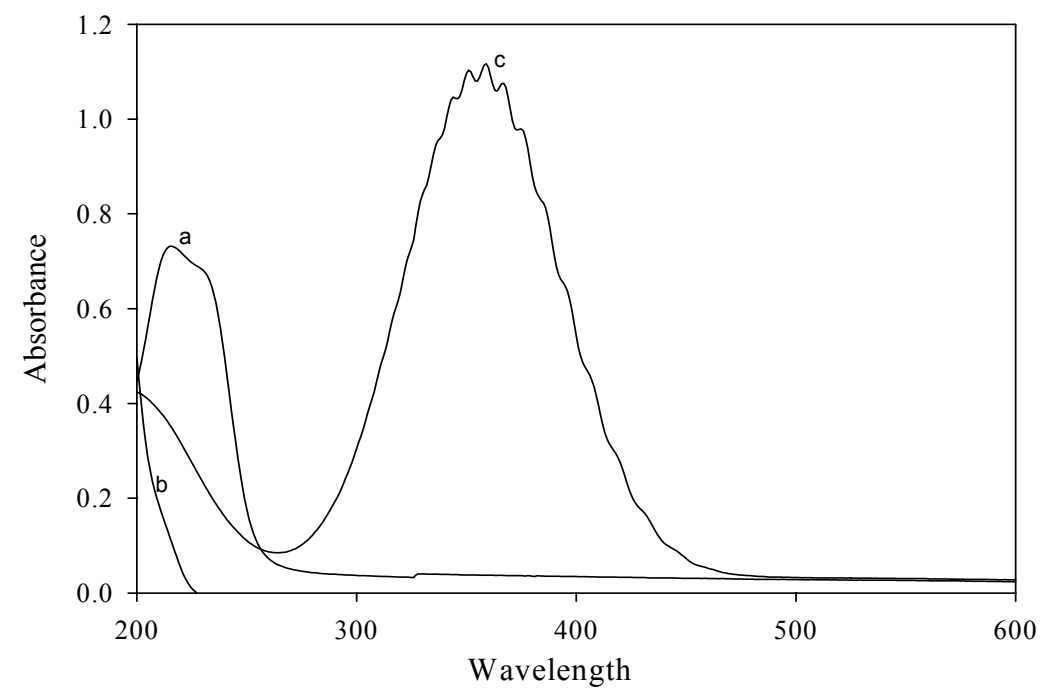

Figure 4.1: UV absorption spectrum of (a) $5.000 \times 10^{-5} \mathrm{M}$ TMTU, (b) $1.000 \times 10^{-4} \mathrm{M}$ TMU and (c) $8.758 \times 10^{-4} \mathrm{M} \mathrm{ClO}_{2}$ showing peaks at $216 \mathrm{~nm}$ and $360 \mathrm{~nm}$ respectively. There is no interference from the substrate or product at $360 \mathrm{~nm}$.

Figure 4.1 shows three superimposed spectra; (a) TMTU ( $\lambda_{\max }=216 \mathrm{~nm}, \epsilon_{\max }=15,646$ $\left.\mathrm{M}^{-1} \mathrm{~cm}^{-1}\right)$, (b) trimethylurea, TMU $\left(\mathrm{C}=1046 \mathrm{M}^{-1} \mathrm{~cm}^{-1}\right.$ at $\lambda=216 \mathrm{~nm}$ ), and (c) chlorine dioxide $\left(\mathrm{C}=397 \mathrm{M}^{-1} \mathrm{~cm}^{-1}\right.$ at $\left.\lambda=216 \mathrm{~nm}\right)$. Chlorine dioxide has negligible absorbance at 
$216 \mathrm{~nm}$. Trimethylurea on the other hand, contributes significantly to the absorbance at $216 \mathrm{~nm}$. Initial rate measurements at $216 \mathrm{~nm}$ were thus only able to deliver accurate information at the beginning of the reaction, before concentrations of trimethylurea and chlorine dioxide accumulated.

The stoichiometric ratio needed for reaction 4.1 was the highest amount of chlorite needed for a fixed amount of TMTU that did not give the yellow chlorine dioxide as a product. To ascertain this, various ratios of chlorite to TMTU were mixed and incubated overnight, after which the reaction mixtures were then spectrophotometrically scanned between 200 and $500 \mathrm{~nm}$. Reaction mixtures that showed positive results for the presence of chlorine dioxide were then subjected to iodometric titrations $[20,21,22]$ to evaluate the excess oxidizing power left after all the TMTU had been consumed. A plot of thiosulfate titer volume vs. initial chlorite concentrations yielded a straight line. Extrapolating to zero titer (chlorite concentration axis), gave an intercept where stoichiometry 4.1 is just satisfied by giving the exact amount of chlorite needed to just consume TMTU without forming $\mathrm{ClO}_{2}$. The final complementary technique in confirming stoichiometry 4.1 involved the data shown in figure 4.3b. If $\mathrm{R}<2$, no chlorine dioxide formation is observed, and induction period (time taken for the production of chlorine dioxide to commence) goes to infinity. Chlorite concentrations were varied for a fixed initial TMTU concentration and induction periods were determined. The intercept, where the induction period is infinity $\left(1 / \mathrm{T}_{\mathrm{ind}}=0\right.$; indicating that no chlorine dioxide formed) constitutes the stoichiometry 4.1. Figure $4.3 \mathrm{~b}$ shows that for a solution with $1.25 \times 10^{-3} \mathrm{M}$ TMTU; 2.55 x $10^{-3} \mathrm{M}$ of chlorite was the intercept value where the induction period was infinity, also confirming the 2:1 stoichiometry. The reaction mixtures were also subjected to 
qualitative analysis for sulfate using barium chloride. Positive results for the presence of sulfate also confirmed that all the sulfur in TMTU ends up as sulfate through a gravimetric analysis. The product, trimethylurea, was identified by its UV spectrum.

\subsubsection{Reaction Dynamics}

The reaction displays a very short induction period followed by a rapid production of chlorine dioxide at $\mathrm{pH}$ ranges of $1-3$. Rapid scan spectra shows an initial decrease in the TMTU peak at $216 \mathrm{~nm}$, with a delayed formation of the $360 \mathrm{~nm}$ (chlorine dioxide) peak as shown in figure 4.2.

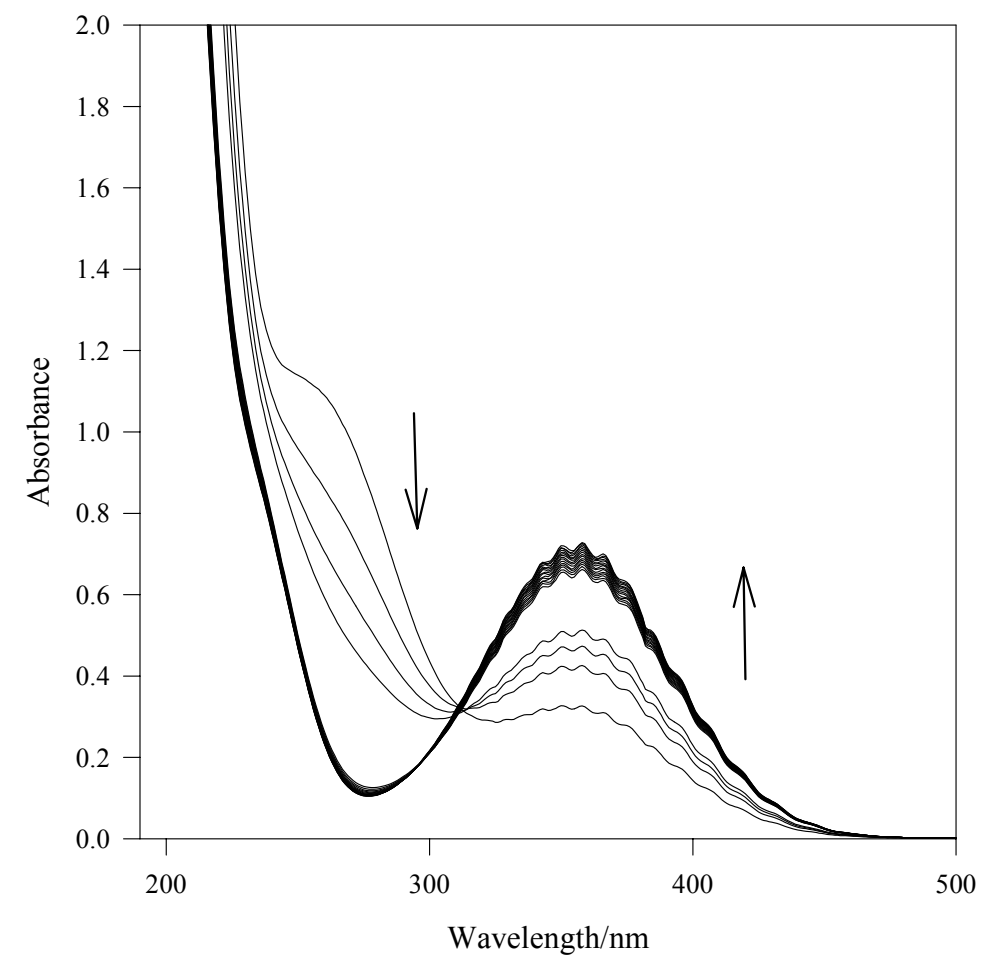

Figure 4.2: Absorbance scans of TMTU during oxidation by chlorite in aqueous acidic medium. Traces were collected every 30 seconds reaction time intervals. $[\mathrm{TMTU}]_{0}=5 \times 10^{-4} \mathrm{M},\left[\mathrm{ClO}_{2}^{-}\right]_{0}=$ $4 \times 10^{-3} \mathrm{M}$, and $\left[\mathrm{HClO}_{4}\right]_{0}=0.1 \mathrm{M}$. 


\subsubsection{Chlorite Dependence}

Figure 4.3a shows the 'clock' nature of this reaction. Initially, there is a quiescent period before chlorine dioxide is formed at $360 \mathrm{~nm}$. This quiescent induction period's length is determined by initial reactant concentrations. In figure $4.3 \mathrm{a}$, the only variable was initial chlorite concentrations. Higher chlorite concentrations gave shorter induction periods, and a much more rapid rate of formation of chlorine dioxide. Figure $4.3 \mathrm{~b}$ shows an inverse, linear relationship between the induction period and initial chlorite concentrations. The induction period can be loosely related to the rate of reaction since the formation of chlorine dioxide should signal a specific event in the reaction's progress. Hence one can assume that chlorite must be involved in the rate-determining step's rate law to the first power.

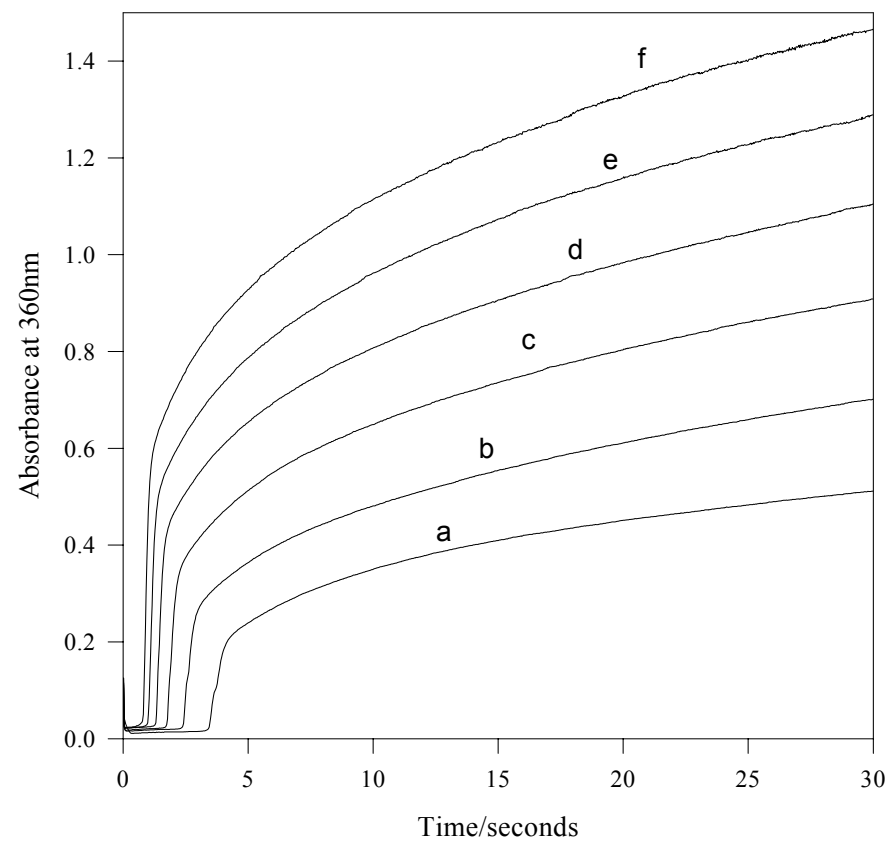

Figure 4.3a: Effect of chlorite in the oxidation of TMTU at $360 \mathrm{~nm}$. Concentration of chlorite is directly proportional to the amount of $\mathrm{ClO}_{2}$ formed and inversely proportional to the induction period at fixed TMTU concentrations: $[\mathrm{TMTU}]_{0}=1.25 \times 10^{-3} \mathrm{M},\left[\mathrm{HClO}_{4}\right]_{0}=0.125 \mathrm{M}, \mathrm{I}_{(\mathrm{NaClO} 4)}=$ $1 \mathrm{M},\left[\mathrm{ClO}_{2}^{-}\right]_{0}=$ (a) $5.00 \times 10^{-3} \mathrm{M}$, (b) $6.25 \times 10^{-3} \mathrm{M}$, (c) $7.50 \times 10^{-3} \mathrm{M}$, (d) $8.75 \times 10^{-3} \mathrm{M}$, (e) $=$ $10.00 \times 10^{-3} \mathrm{M}$, (f) $11.25 \times 10^{-3} \mathrm{M}$. 


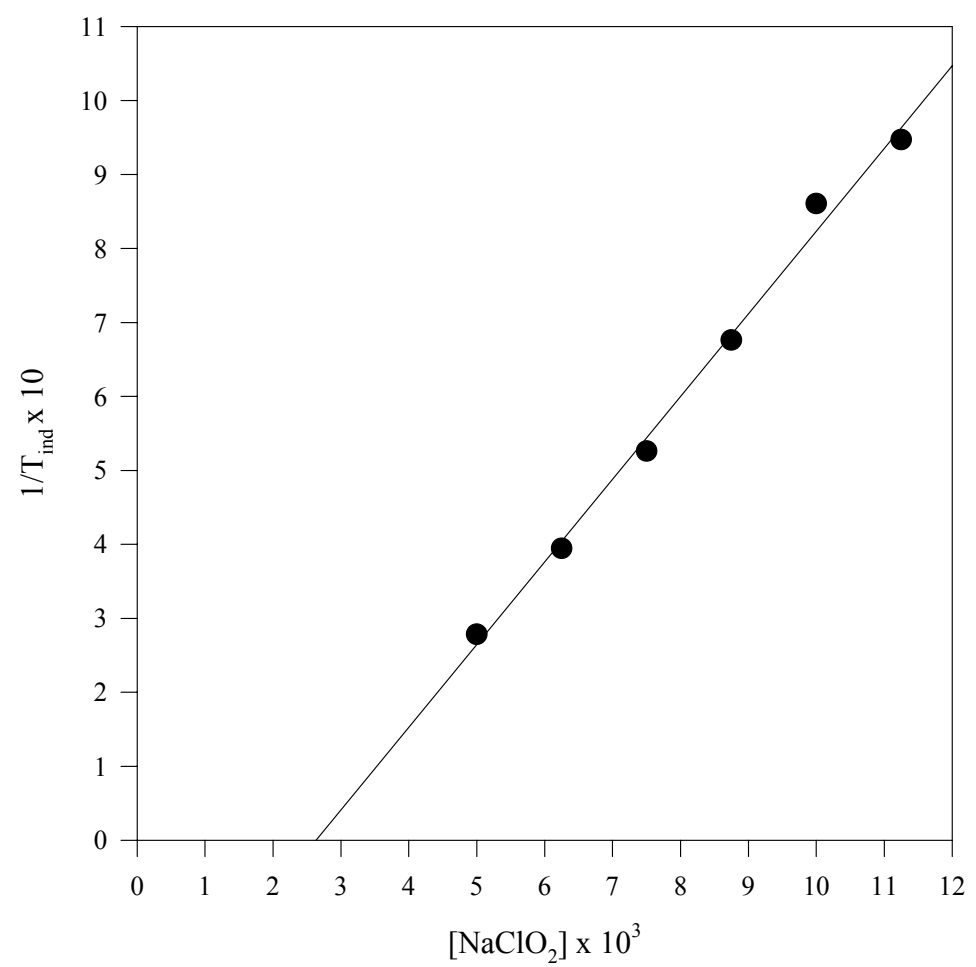

Figure 4.3b: Plot of $1 /$ Tind vs $\left[\mathrm{ClO}_{2}^{-}\right]$using stochiometric excess of chlorite, showing that an inverse relationship exists for a long range of chlorite concentrations. [TMTU] $]_{0}=1.25 \times 10^{-3} \mathrm{M}$, $\left[\mathrm{HClO}_{4}\right]_{0}=0.125 \mathrm{M}, \mathrm{I}_{(\mathrm{NaClO} 4)}=1 \mathrm{M}$.

\subsubsection{Acid Dependence}

In contrast, the reaction showed a very complex acid dependence as shown in figures $4.4 \mathrm{a}$ and $4.4 \mathrm{~b}$. Experiments in figure $4.4 \mathrm{a}$ were carried out at low acid environments within the $\mathrm{pH}$ range of $1.6-2.3$. Acid does not seem to affect the induction period under these conditions, but rather increases the rate and amount of chlorine dioxide formed after the induction period. Experiments in figure 4.4b, on the other hand, were done at high acid environments within the $\mathrm{pH}$ range of $0.7-1.0$. Although acid still does not affect the induction period in this range, it now retards chlorine dioxide formation after the induction period. 


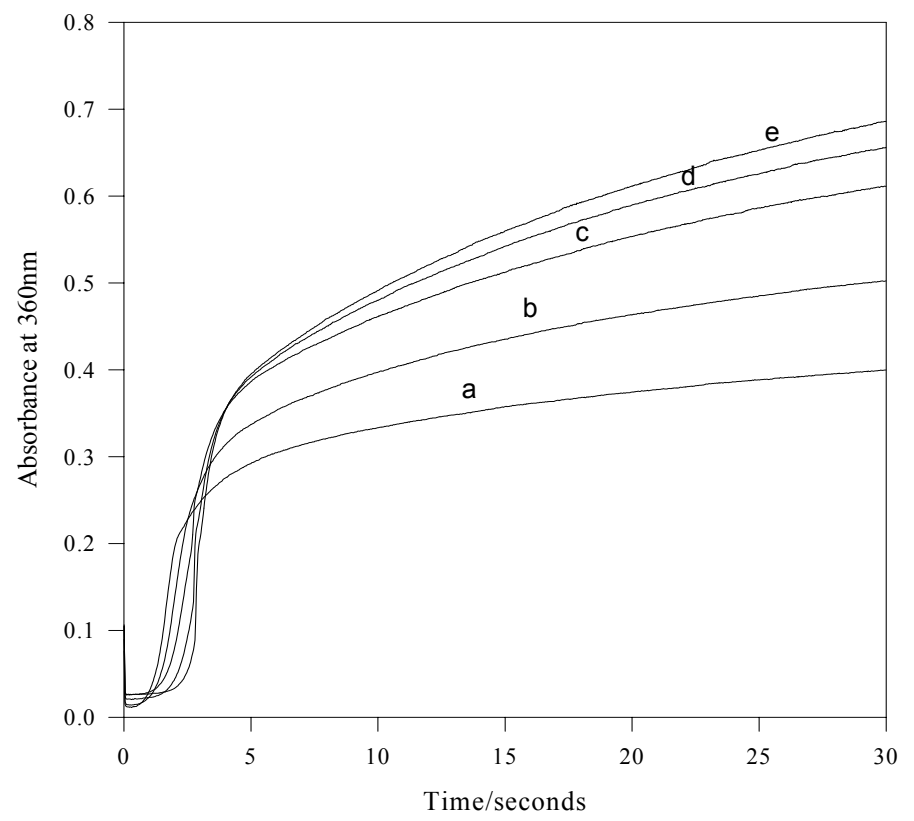

Figure 4.4a: Absorbance traces at $360 \mathrm{~nm}$ showing effect of low acid concentrations on the oxidation of TMTU by chlorite. In this case there is no well defined induction period and the amount of chlorine dioxide formed is directly proportional to the amount of acid. [TMTU] $]_{0}=$ $1.25 \times 10^{-3} \mathrm{M},\left[\mathrm{ClO}_{2}^{-}\right]_{0}=5.00 \times 10^{-3} \mathrm{M}, \mathrm{I}_{(\mathrm{NaClO} 4)}=1 \mathrm{M},\left[\mathrm{HClO}_{4}\right]_{0}=$ (a) $0.005 \mathrm{M}$, (b) $=0.010 \mathrm{M}$, (c) $0.015 \mathrm{M}$, (d) $0.020 \mathrm{M}$, (e) $0.025 \mathrm{M}$.

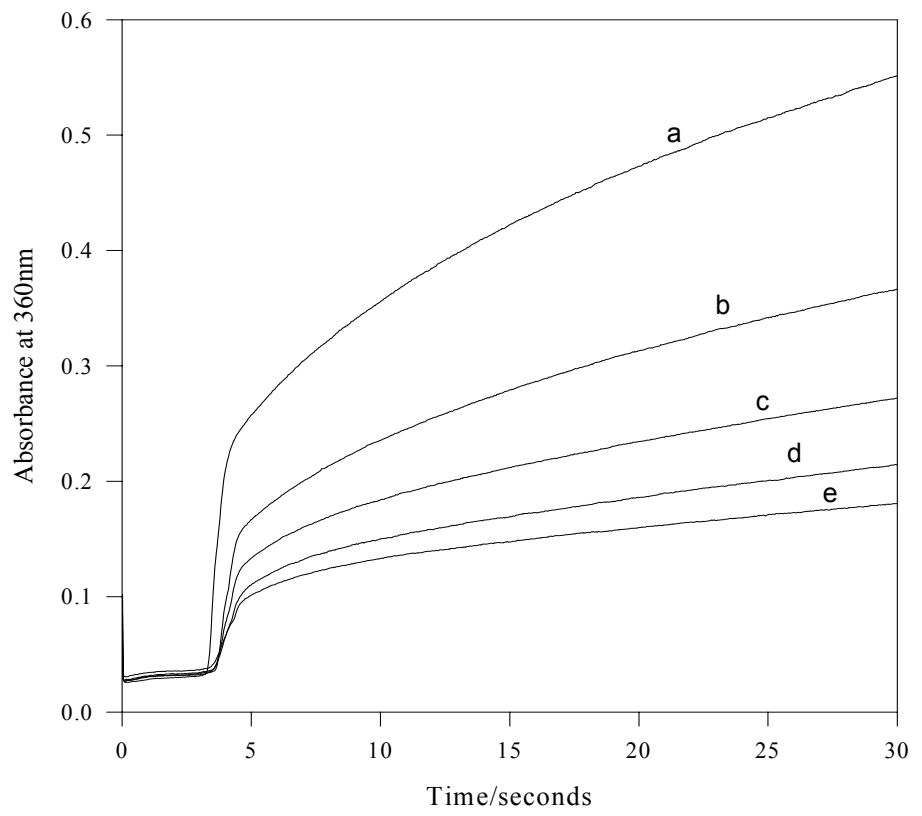

Figure 4.4b: Absorbance traces at $360 \mathrm{~nm}$ showing effect of high acid concentrations on the oxidation of TMTU by chlorite. In this case there is a well defined induction period which in not affected by the amount of acid. Concentration of acid is inversely proportional to the amount of chorine dioxide formed. The amount of $[\mathrm{TMTU}]_{0}=1.25 \times 10^{-3} \mathrm{M},\left[\mathrm{ClO}_{2}{ }^{-}\right]_{0}=5.00 \times 10^{-3} \mathrm{M}$, $\mathrm{I}_{(\mathrm{NaClO} 4)}=1 \mathrm{M},\left[\mathrm{HClO}_{4}\right]_{0}=$ (a) $0.1 \mathrm{M}$, (b) $0.2 \mathrm{M}$, (c) $0.3 \mathrm{M}$, (d) $0.4 \mathrm{M}$, (e) $0.5 \mathrm{M}$. 


\subsubsection{TMTU dependence}

Varying initial TMTU concentrations at a fixed initial chlorite concentration showed a lengthening of induction period, and an increase in the rate and amount of chlorine dioxide produced, with increasing TMTU concentrations. This is to be expected if production of chlorine dioxide signifies a specific progress in the oxidation of TMTU, this position will require a longer time period for it to be attained for higher TMTU concentrations, for a fixed amount of oxidant, chlorite.

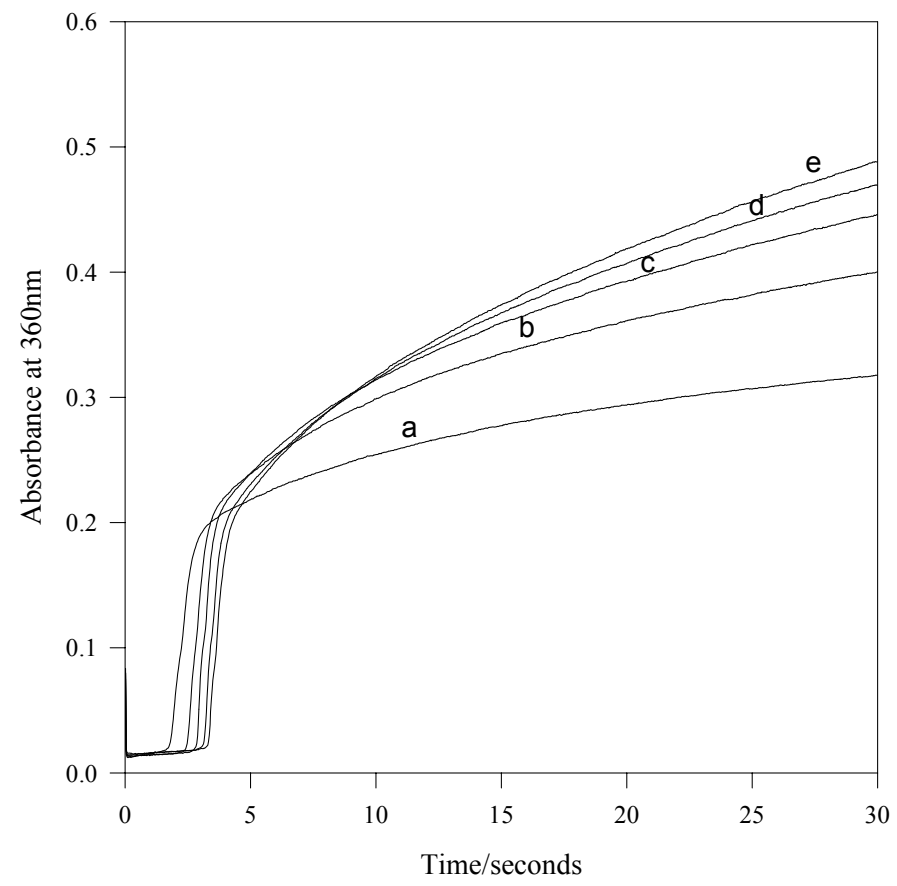

Figure 4.5: Effect of varying TMTU on the formation of chlorine dioxide. The amount of chlorine dioxide formed is directly proportional to the amount $\left[\mathrm{TMTU}_{0} .\left[\mathrm{ClO}_{2}{ }^{-}\right]_{0}=5.00 \times 10^{-3} \mathrm{M}\right.$, $\left[\mathrm{HClO}_{4}\right]_{0}=0.125 \mathrm{M}, \mathrm{I}_{(\mathrm{NaClO} 4)}=1 \mathrm{M},[\mathrm{TMTU}]_{0}=$ (a) $2.5 \times 10^{-4} \mathrm{M}$, (b) $5.0 \times 10^{-4} \mathrm{M}$, (c) $7.5 \times 10^{-}$ ${ }^{4} \mathrm{M}$, (d) $10.0 \times 10^{-4} \mathrm{M}$, (e) $12.5 \times 10^{-4} \mathrm{M}$.

\subsubsection{Consumption of TMTU}

The reaction could be followed spectrophotometrically by monitoring the absorbance peak, $\lambda_{\max }$, of TMTU at $216 \mathrm{~nm}$. The product of the reaction, trimethylurea, has a substantial absorbance at $216 \mathrm{~nm}$ as shown in figure 4.1 and thus data such as those 
shown in figure 4.6a are only useful for the evaluation of reaction rates at the beginning of the reaction before trimethylurea, trimethylthiourea intermediates, and chlorine dioxide start to assert themselves. The observed acid effects are also dependent on acid strength.

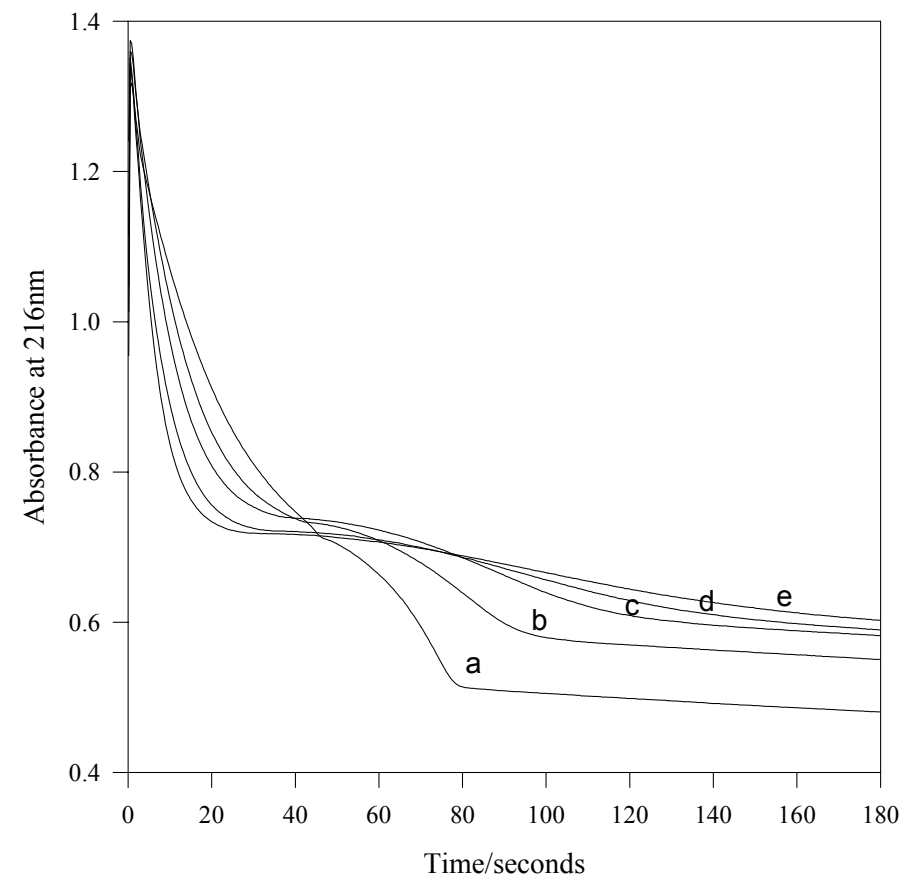

Figure 4.6a: Effect of acid on the depletion of TMTU in its oxidation by chlorite monitored at $216 \mathrm{~nm}$. The reaction displays two-stage kinetics at lower acid concentrations. [TMTU] $]_{0}=7.5 \mathrm{x}$ $10^{-5} \mathrm{M},\left[\mathrm{ClO}_{2}^{-}\right]_{0}=5.00 \times 10^{-4} \mathrm{M}, \mathrm{I}_{(\mathrm{NaClO} 4)}=1 \mathrm{M},\left[\mathrm{HClO}_{4}\right]_{0}=$ (a) $0.1 \mathrm{M}$, (b) $0.2 \mathrm{M}$, (c) $0.3 \mathrm{M}$, (d) 0.4 $\mathrm{M}$, (e) $0.5 \mathrm{M}$.

Figure 4.6a shows that a monotonic decay in absorbance is observed at $216 \mathrm{~nm}$ in high acid environments; whereas in low acid environments, a sigmoidal autocatalytic decay is observed as shown in figure 4.6b. The reaction appears to occur in two stages: an initial simple decay (high acid) followed by an autocatalytic decay (see figure 4.6a, trace a). However, the absorbance observed at about $20 \mathrm{~s}$ into the reaction is dominated by trimethylthiourea oxo-acids and other species. Thus, it is no longer possible to attribute 
the absorbance to a single dominant species. Oxy-chlorine species also contribute to the absorbance at this wavelength as shown in figure 4.1.

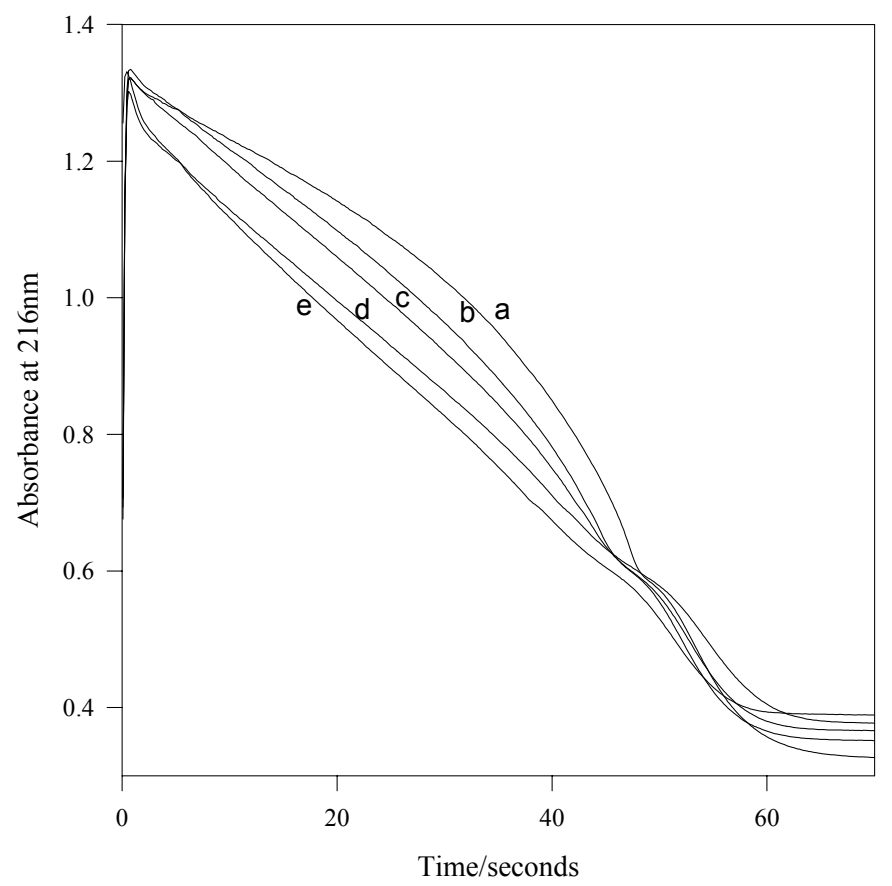

Figure 4.6b: Effect of acid on the depletion of TMTU in its oxidation by chlorite monitored at $216 \mathrm{~nm} .[\mathrm{TMTU}]_{0}=7.5 \times 10^{-5} \mathrm{M},\left[\mathrm{ClO}_{2}{ }^{-}\right]_{0}=5.00 \times 10^{-4} \mathrm{M}, \mathrm{I}_{(\mathrm{NaClO} 4)}=1 \mathrm{M},\left[\mathrm{HClO}_{4}\right]_{0}=$ (a) 0.005 $\mathrm{M}$, (b) $0.010 \mathrm{M}$, (c) $0.015 \mathrm{M}$, (d) $0.020 \mathrm{M}$, (e) $0.025 \mathrm{M}$.

In conditions of high acid, coupled with high excess of oxidant; $\mathrm{R} \geq 10$; pseudo-first order kinetics are never attained (see figure 4.6c). Very low acid concentrations show sigmoidal autocatalytic decay kinetics (not shown), increasing acid concentrations changes the kinetics to a straight line zero order type decay (trace a), which gives way to standard concave decay kinetics at high acid environments (traces d-e). The first stage of the reaction is catalyzed by high acid concentrations, while low acid concentrations catalyzes the second stage, since, in high acid environments, the intermediate formed lingers much longer (is more stable) before forming products. 


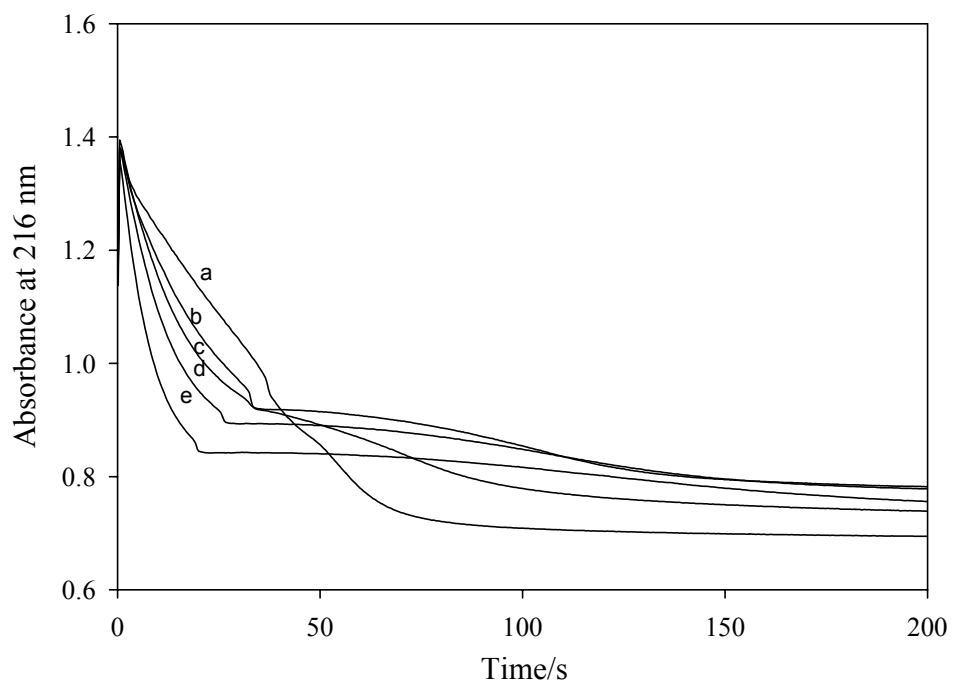

Figure 4.6c: Effect of acid on the depletion of TMTU in its oxidation by chlorite monitored at $248 \mathrm{~nm}$. The data still displays two-state kinetics, with the rate of the first stage being catalyzed by high acid concentrations (oxidations by $\mathrm{HClO}_{2}$ ), while the second stage is catalyzed by lower acid concentrations (oxidations by both $\mathrm{HClO}_{2}$ and $\mathrm{ClO}_{2}^{-}$). $[\mathrm{TMTU}]_{0}=5.00 \times 10^{-5} \mathrm{M},\left[\mathrm{ClO}_{2}{ }^{-}\right]_{0}=$ $5.00 \times 10^{-4} \mathrm{M}, \mathrm{I}_{(\mathrm{NaClO} 4)}=1 \mathrm{M},\left[\mathrm{HClO}_{4}\right]_{0}=$ (a) $0.025 \mathrm{M}$, (b) $0.075 \mathrm{M}$, (c) $0.1 \mathrm{M}$, (d) $0.15 \mathrm{M}$, (e) 0.2 M.

Figure 4.7a shows the effect of chlorite at $216 \mathrm{~nm}$. Chlorite appears to catalyze the initial stages of the reaction, although the later stages of the reaction appear a little confusing. The final baseline absorbance observed is directly proportional to the initial chlorite concentrations, thus lending credence to the fact that oxy-chlorine species have a substantial contribution to the absorbance observed at $216 \mathrm{~nm}$. The criss-crossing of the absorbance traces is due to the fact that higher chlorite concentrations start with higher initial absorbances, and since chlorite catalyzes the reaction, those traces with a higher absorbance will have to cross those traces at lower chlorite concentrations. The catalytic effect of chlorite is discernible by noting the time taken for the final absorbance reading to be attained, and not by the value of the final absolute reading itself. For example, the 
final absorbance is reached in $42 \mathrm{~s}$ for an initial chlorite concentration of $0.001 \mathrm{M}$, while for $0.0005 \mathrm{M}$ chlorite the final absorbance is reached in $125 \mathrm{~s}$.

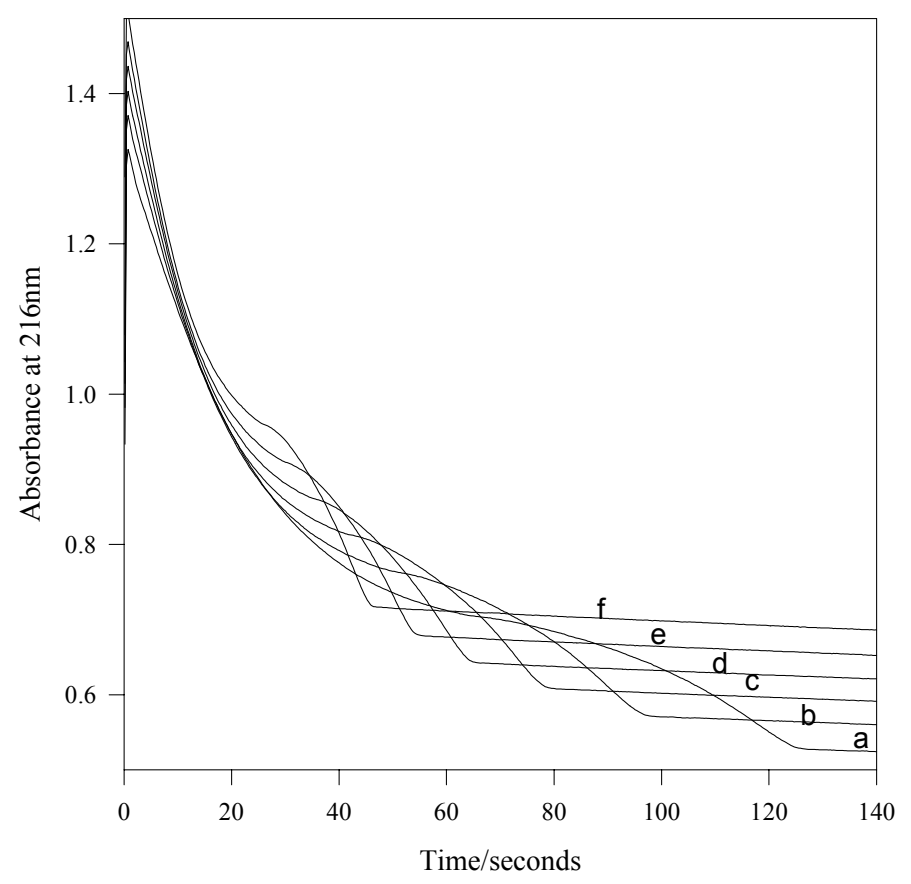

Figure 4.7a: Effect of chlorite on the depletion of TMTU in its oxidation by chlorite monitored at $216 \mathrm{~nm}$. The data displays two-stage kinetics. The products of the TMTU oxidation give residual absorbance as can be seen in the absorbance-time data. [TMTU $]_{0}=7.5 \times 10^{-5} \mathrm{M},\left[\mathrm{HClO}_{4}\right]_{0}=$ $0.125 \mathrm{M}, \mathrm{I}_{(\mathrm{NaClO} 4)}=1 \mathrm{M},\left[\mathrm{ClO}_{2}^{-}\right]_{0}=$ (a) $5.0 \times 10^{-4} \mathrm{M}$, (b) $6.0 \times 10^{-4} \mathrm{M}$, (c) $7.0 \times 10^{-4} \mathrm{M}$, (d) $8.0 \mathrm{x}$ $10^{-4} \mathrm{M}$, (e) $9.0 \times 10^{-4} \mathrm{M}$, (f) $10.0 \times 10^{-4} \mathrm{M}$.

Figure $4.7 \mathrm{~b}$ shows two traces superimposed, one at $360 \mathrm{~nm}$ and the other at $216 \mathrm{~nm}$. The absorbance readings at $360 \mathrm{~nm}$ were magnified $5 \mathrm{X}$ so that they could be on the same scale as those at $216 \mathrm{~nm}$. In figure $4.7 \mathrm{~b}$, one notices that chlorine dioxide production commences before the end of the first stage of the reaction. This is only possible if the reactions of chlorine dioxide with TMTU and its intermediates are slow enough to allow for their co-existence. 


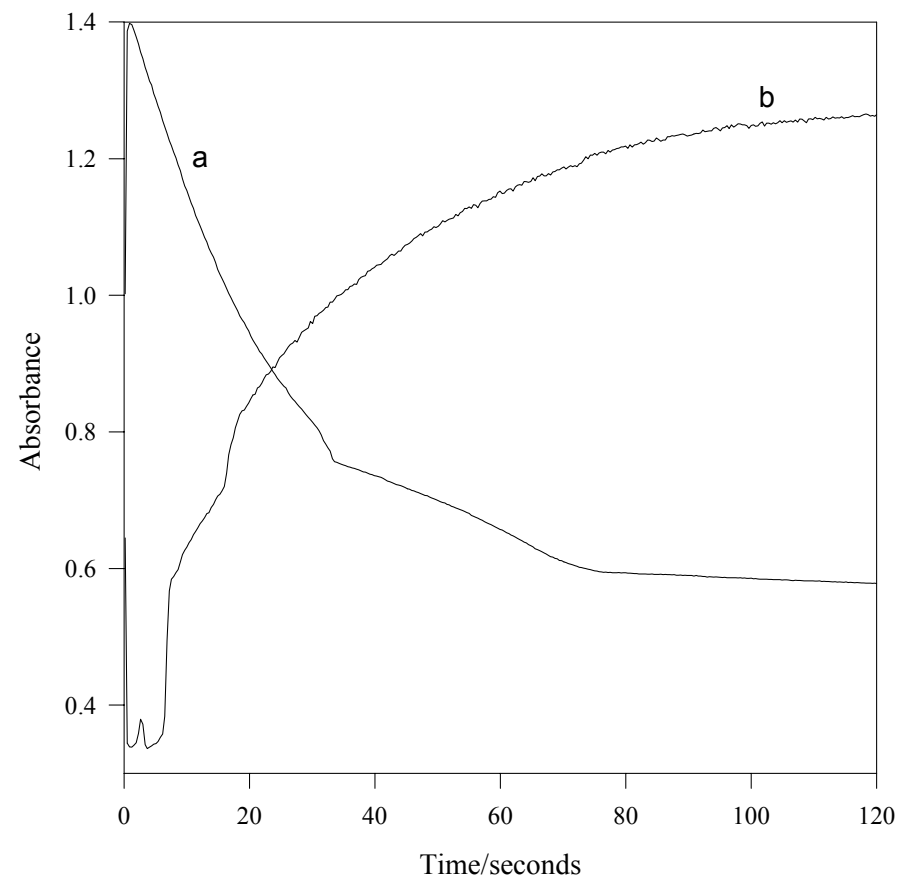

Figure 4.7b: Two traces superimposed at (a) $216 \mathrm{~nm}$ and (b) $360 \mathrm{~nm}$. $\left[\mathrm{ClO}_{2}{ }^{-}\right]_{0}=5.00 \times 10^{-4} \mathrm{M}$, $[\mathrm{TMTU}]_{0}=5.0 \times 10^{-5} \mathrm{M},\left[\mathrm{HClO}_{4}\right]_{0}=0.125 \mathrm{M},(\mathrm{NaClO})=1 \mathrm{M}$.

The data in figures $4.8 \mathrm{a}$ and $4.8 \mathrm{~b}$ are important in interpretation of the absorbance spectra. Due to the very high absorptivity coefficient of TMTU at $216 \mathrm{~nm}$, only micromolar quantities are needed for optical density analysis. Thus in all the traces shown, oxidant to reductant ratio, $\mathrm{R}=\left[\mathrm{ClO}_{2}^{-}\right]_{0} /[\mathrm{TMTU}]_{0}$ is very high and lies between 4.0 and 20. All TMTU will be quantitatively converted to the urea analogue with an ever increasing baseline final absorbance as observed in figure 4.8a. A plot of the final absorbance vs. initial TMTU concentrations will give a straight line with a positive intercept, which corresponds to the contributions in absorbance from all other species in the reaction mixture which are not from the TMU product. These data were utilized further in the deduction of the stoichiometry and mechanism. The follow up plot in figure $4.8 \mathrm{~b}$ shows a linear dependence on the initial rate with TMTU as expected. The intercept of this plot was kinetically indistinguishable from zero. 


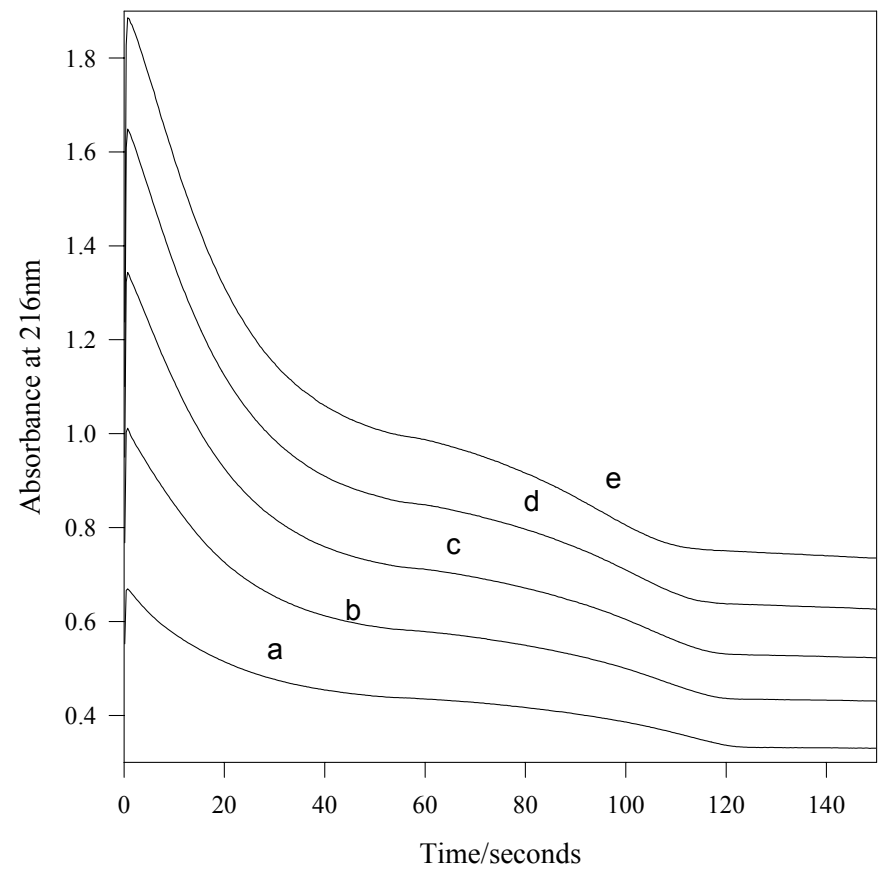

Figure 4.8a: Depletion of TMTU in its oxidation by chlorite. The data also displays two-stage kinetics. $\left[\mathrm{ClO}_{2}^{-}\right]_{0}=5.00 \times 10^{-4} \mathrm{M},\left[\mathrm{HClO}_{4}\right]_{0}=0.125 \mathrm{M},{ }_{(\mathrm{NaClO} 4)}=1 \mathrm{M},[\mathrm{TMTU}]_{0}=$ (a) $2.5 \times 10^{-5} \mathrm{M}$, (b) $5.0 \times 10^{-5} \mathrm{M}$, (c) $7.5 \times 10^{-5} \mathrm{M}$, (d) $10.0 \times 10^{-5} \mathrm{M}$, (e) $12.5 \times 10^{-5} \mathrm{M}$.

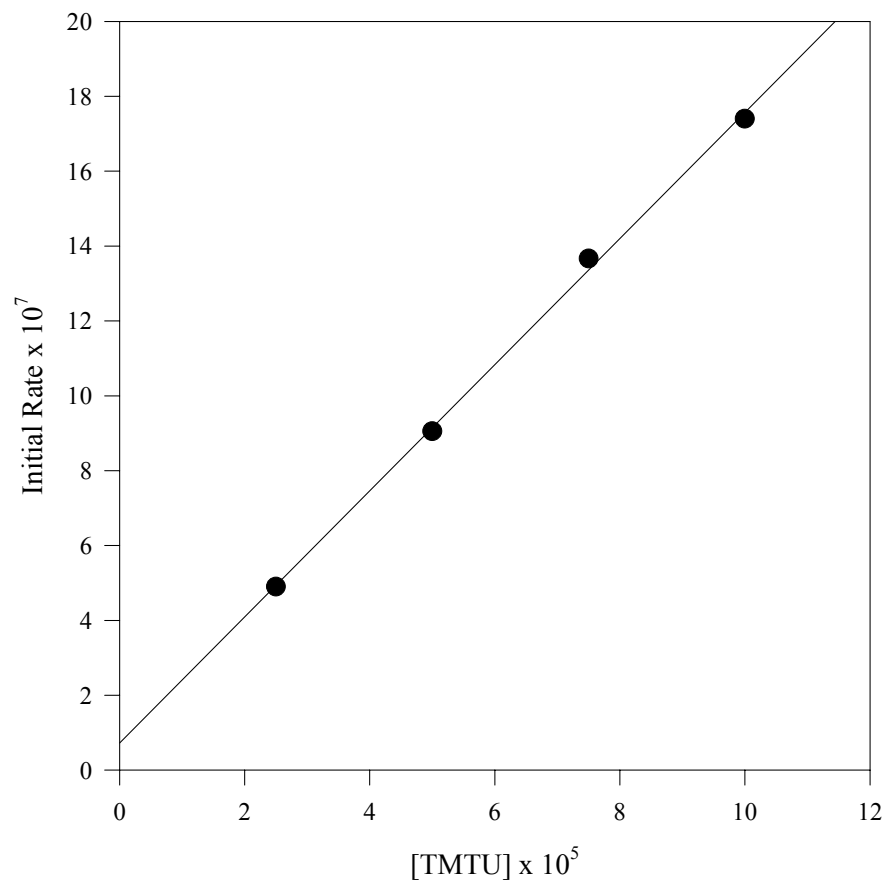

Figure 4.8b: Effect of initial TMTU concentration on the initial rate of consumption of TMTU. There is a solid linear dependence. $\left[\mathrm{ClO}_{2}^{-}\right]_{0}=5.00 \times 10^{-4} \mathrm{M},\left[\mathrm{HClO}_{4}\right]_{0}=0.125 \mathrm{M}, \mathrm{I}_{(\mathrm{NaClO} 4)}=1 \mathrm{M}$. 


\subsubsection{Oxidations by chlorine dioxide}

Oxidation reactions of chlorine dioxide are comparable in rate with those of chlorite. This renders the chlorite/TMTU reactions very complex and difficult to interpret because formation of chlorine dioxide does not imply that all the reducing species in the reaction medium have been completely oxidized as shown in figure $4.7 \mathrm{~b}$.

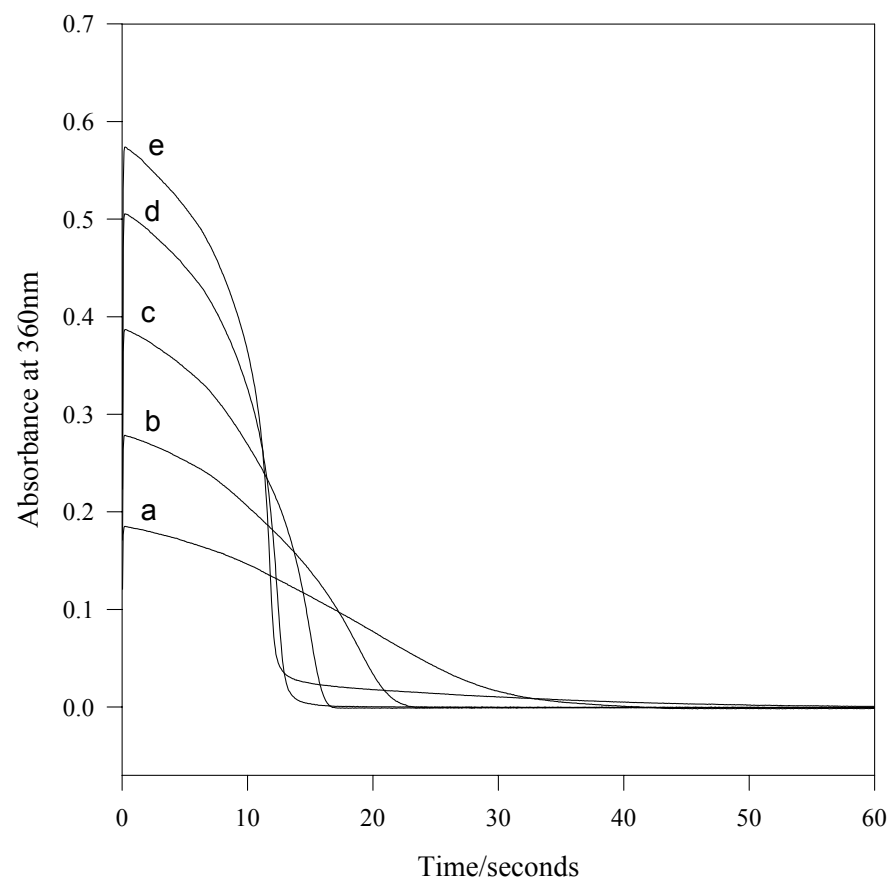

Figure 4.9a: Oxidation of TMTU by chlorine dioxide monitored at $360 \mathrm{~nm}$, with oxidant to reductant ratios below stoichiometric amount. A single sigmoidal decay for the consumption of chlorine dioxide is observed with all the chlorine dioxide being depleted at the end of the reaction. $[\mathrm{TMTU}]_{0}=2.5 \times 10^{-4} \mathrm{M},\left[\mathrm{H}^{+}\right]_{0}=0.125 \mathrm{M}, \mathrm{I}_{\mathrm{NaClO} 4}=1 \mathrm{M},\left[\mathrm{ClO}_{2}\right]_{0}=$ (a) $2.0 \times 10^{-4} \mathrm{M}$, (b) $3.0 \times 10^{-4} \mathrm{M}$, (c) $4.0 \times 10^{-4} \mathrm{M}$, (d) $5.0 \times 10^{-4} \mathrm{M}$, and (e) $6.0 \times 10^{-4} \mathrm{M}$.

Figure $4.9 \mathrm{a}$ and $4.9 \mathrm{~b}$ shows a series of experiments with varying chlorine dioxide concentrations. Each trace shows a sigmoidal autocatalytic consumption with an ever increasing rate of consumption of chlorine dioxide with time. In excess TMTU zero order type decay kinetics are observed in the consumption of chlorine dioxide (see figure 4.9a trace a), which give way to sigmoidal autocatalytic kinetics as chlorine dioxide concentrations are increased. 


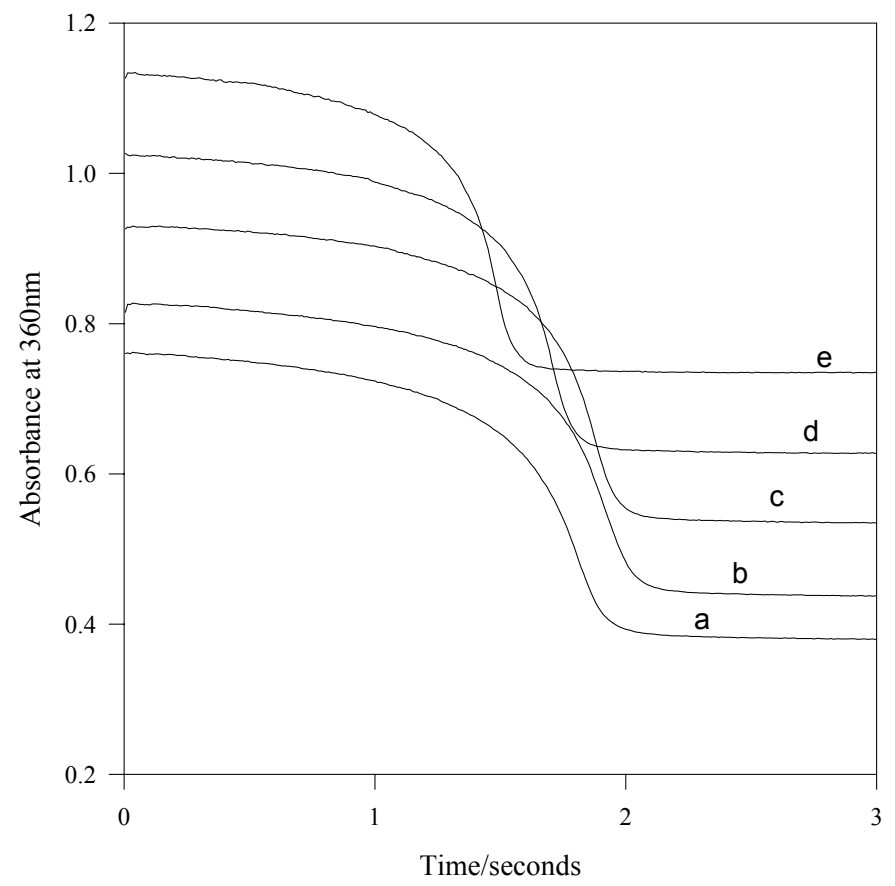

Figure 4.9b: Effect of chlorine dioxide in the depletion of TMTU. Rate of depletion of TMTU increases with increase in chlorine dioxide concentrations. [TMTU $]_{0}=2.5 \times 10^{-4} \mathrm{M},\left[\mathrm{HClO}_{4}\right]_{0}=$ $0.125 \mathrm{M}, \mathrm{I}_{(\mathrm{NaClO} 4)}=1 \mathrm{M},\left[\mathrm{ClO}_{2}\right]_{0}=$ (a) $7.0 \times 10^{-4} \mathrm{M}$, (b) $8.0 \times 10^{-4} \mathrm{M}$, (c) $9.0 \times 10^{-4} \mathrm{M}$, (d) $10.0 \mathrm{x}$ $10^{-4} \mathrm{M}$, (e) $11.0 \times 10^{-4} \mathrm{M}$.

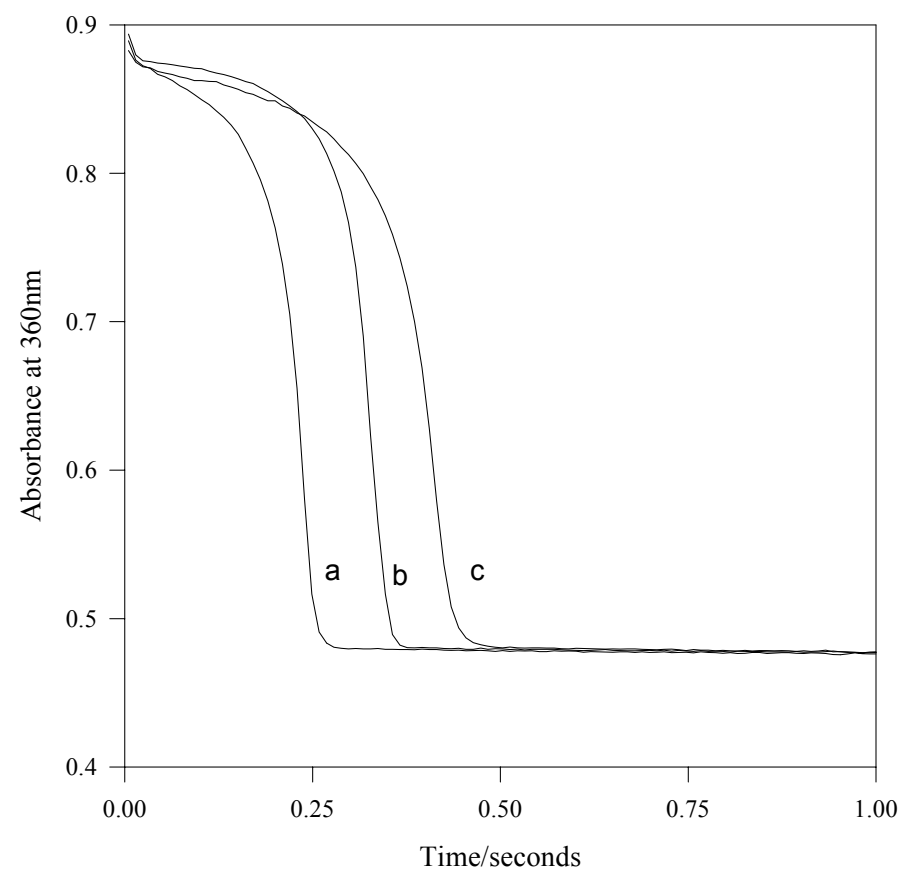

Figure 4.10: Effect of low acid concentrations on the oxidation of TMTU by chlorine dioxide. Rate of depletion of TMTU decreases with increase in acid concentrations. [TMTU] $]_{0}=2.5 \times 10^{-4}$ $\mathrm{M},\left[\mathrm{ClO}_{2}\right]_{0}=1.00 \times 10^{-3} \mathrm{M}, \mathrm{I}_{(\mathrm{NaClO} 4)}=1 \mathrm{M},\left[\mathrm{HClO}_{4}\right]_{0}=$ (a) $0.005 \mathrm{M}$, (b) $0.010 \mathrm{M}$, (c) $0.015 \mathrm{M}$. 
Figure 4.9b shows experiments done in excess chlorine dioxide. Increase in acid concentrations inhibits the reaction by lengthening the induction period before rapid oxidation commences as shown in figure 4.10. Previous studies had alluded to the fact that most chlorine dioxide oxidations proceed first through the formation of chlorite, which then conducts the bulk of the oxidations [20].Data shown in figure 4.11, shows the effect of very minute amounts of chlorite on the rate of oxidation of TMTU by chlorine dioxide. One notes that the addition of chlorite catalyzes the reaction visibly even at micromolar concentrations. Figure 4.11 also shows a control experiment in which no chlorite was added. The parallel nature of the absorbance traces with varying chlorite concentrations shows that chlorite catalyses the reaction from $t=0$ but is in itself, not an autocatalytic species. Addition of chloride ions lengthens the induction period and inhibits the reaction (data not shown).

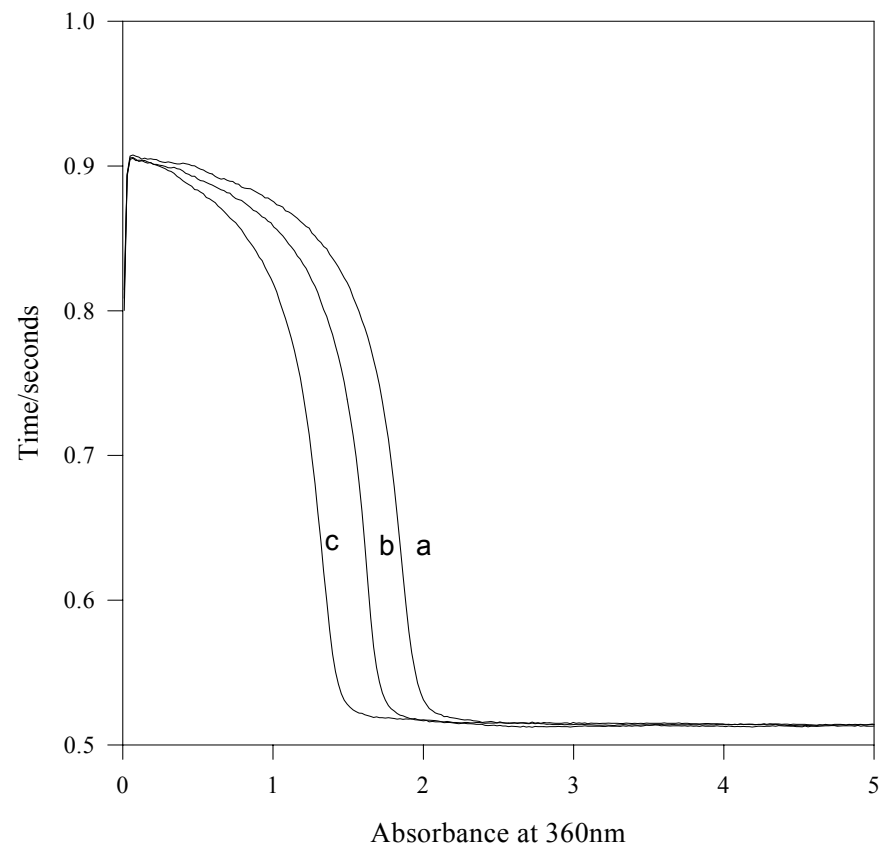

Figure 4.11: Effect of chlorite on the oxidation of TMTU by chlorine dioxide. [TMTU $]_{0}=2.5 \mathrm{x}$ $10^{-4} \mathrm{M},\left[\mathrm{ClO}_{2}\right]_{0}=1.00 \times 10^{-3} \mathrm{M},\left[\mathrm{HClO}_{4}\right]_{0}=0.125 \mathrm{M}, \mathrm{I}_{(\mathrm{NaClO} 4)}=1 \mathrm{M},\left[\mathrm{ClO}_{2}{ }^{-}\right]=$(a) $0.0 \times 10^{-5} \mathrm{M}$, (b) $1.0 \times 10^{-5} \mathrm{M}$, (c) $1.5 \times 10^{-5} \mathrm{M}$. 


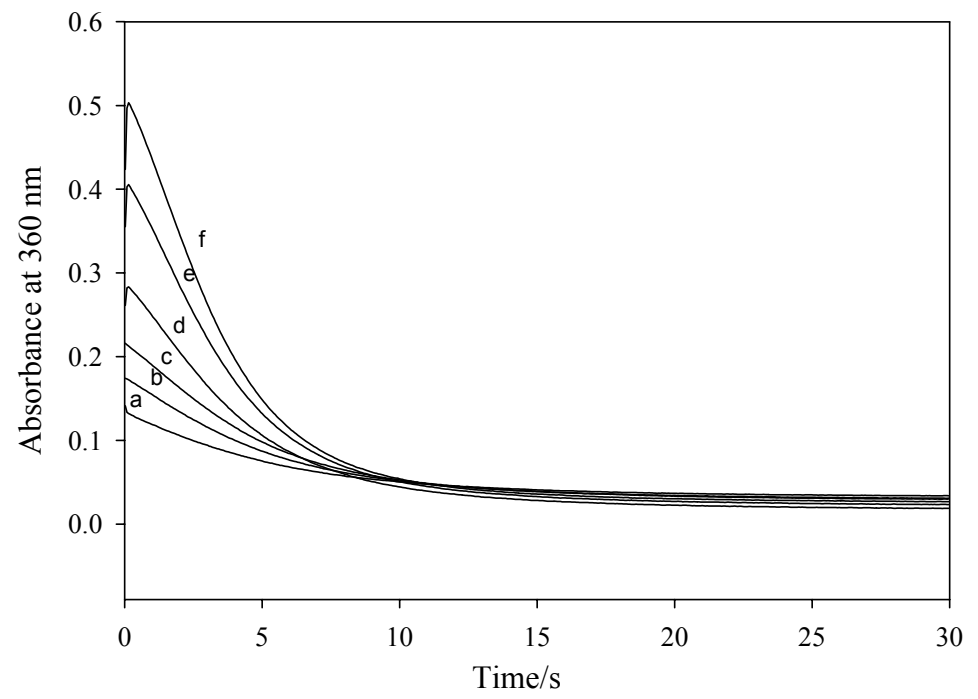

Figure 4.12a: Chlorine dioxide variation in high excess of TMTU at intermediate acid concentrations. Under these acid conditions, all $\mathrm{Cl}(\mathrm{III})$ species are in the chlorous acid form, and the TMTU is mostly unprotonated. $[\mathrm{TMTU}]_{0}=0.01 \mathrm{M},\left[\mathrm{H}^{+}\right]=0.10 \mathrm{M},\left[\mathrm{ClO}_{2}\right]_{0}=$ (a) $4.50 \times 10^{-5} \mathrm{M}$, (b) $9.00 \times 10^{-5} \mathrm{M}$, (c) $1.35 \times 10^{-4} \mathrm{M}$, (d) $2.70 \times 10^{-4} \mathrm{M}$, (e) $3.60 \times 10^{-4} \mathrm{M}$, and (f) $=4.50 \times 10^{-4} \mathrm{M}$

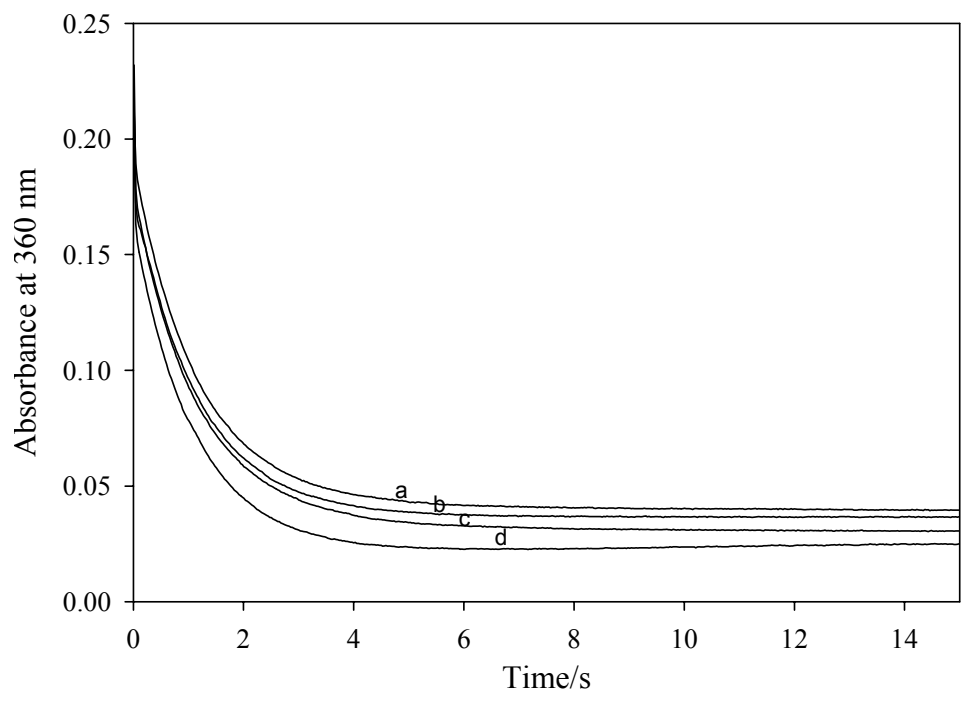

Figure 4.12b: Pseudo-first order kinetics in high excess of TMTU and high acid concentrations. Under these acid concentrations both $\mathrm{Cl}(\mathrm{III})$ species and TMTU are protonated. $\left[\mathrm{ClO}_{2}\right]_{0}=1.8 \mathrm{x}$ $10^{-4} \mathrm{M},\left[\mathrm{HClO}_{4}\right]_{0}=0.10 \mathrm{M},[\mathrm{TMTU}]_{0}=$ (a) $0.0175 \mathrm{M}$, (b) $0.0185 \mathrm{M}$, (c) $0.0190 \mathrm{M}$, and (d) $0.0195 \mathrm{M}$ 
Figures $4.12 \mathrm{a}$ and $4.12 \mathrm{~b}$ show a series of experiments in which $[\mathrm{TMTU}]_{0}>>\left[\mathrm{ClO}_{2}\right]_{0}$. In figure $4.12 \mathrm{a}$ the acid concentrations were fixed at $0.10 \mathrm{M}$. A monotonic decrease in chlorine dioxide concentrations was observed, suggesting that the nonlinear autocatalytic catalysis is generated by the oxychlorine species and not by TMTU. A plot of initial rate vs. $[\mathrm{TMTU}]_{0}$ for these traces is linear, indicating that the reaction of chlorine dioxide and TMTU is first order in [TMTU]. In figure $4.12 \mathrm{~b}$ acid concentrations were elevated to 1.0 M, and pseudo-first order kinetics prevailed. The apparent rate constant evaluated was invariant with changes in the initial TMTU concentration, indicating that the reaction became zero order in [TMTU] at such high acid concentrations. From the data in figure 4.12a, the bimolecular rate constant for the reaction between chlorine dioxide and TMTU was evaluated to be $16 \pm 3.0 \mathrm{M}^{-1} \mathrm{~s}^{-1}$.

\subsection{MECHANISM}

\subsubsection{Acid dependence}

While the reaction dynamics of the oxidation of TMTU by chlorite appear to be quite complex, they can be easily rationalized by two rapid protolytic reactions that involve the protonation of the chlorite anion and the TMTU molecule. Acid concentrations of the reaction environment will then determine the dominant and active species and the subsequent global dynamics observed. The important and confusing data that needs to be rationalized are contained in figures $4.4 \mathrm{a}, 4.4 \mathrm{~b}, 4.6 \mathrm{a}, 4.6 \mathrm{~b}$ and $4.6 \mathrm{c}$. The reaction dynamics appear to run counter to the expected acid catalysis observed with all oxyhalogen species. 


$$
\begin{gathered}
\mathrm{ClO}_{2}^{-}+\mathrm{H}^{+} \rightleftharpoons \mathrm{HClO}_{2} ; \quad K_{\mathrm{a} 1}^{-1} \\
\mathrm{Me}_{2} \mathrm{~N}(\mathrm{NHMe}) \mathrm{C}=\mathrm{S}+\mathrm{H}^{+} \rightleftharpoons\left[\mathrm{Me}_{2} \mathrm{~N}(\mathrm{NHMe}) \mathrm{C}=\mathrm{S}-\mathrm{H}\right]^{+},\left([\mathrm{TMTU}-\mathrm{H}]^{+}\right) ; K_{\mathrm{b}}
\end{gathered}
$$

From these two protonation reactions, four rate-determining step reactions can be derived in which the primary objective is the oxidation of TMTU by chlorite. The viability of these four reactions will depend on the $\mathrm{pH}$, and no conditions exist in which all four reactions will be simultaneously feasible. At any $\mathrm{pH}$ conditions, not more than two of these reactions will dominate. Thus, it should be easy to evaluate the rate constants without involving too many variables. The first intermediate, after two-electron oxidation of TMTU is the sulfenic acid; $\mathrm{Me}_{2} \mathrm{~N}(=\mathrm{NMe}) \mathrm{C}-\mathrm{SOH}$, TMTUSOH. The one-electron oxidation of TMTU to produce thiyl radicals is mediated by one-electron metal ion oxidants such as $\mathrm{Cu}^{2+}$ and $\mathrm{Fe}^{3+}[23,24]$. Since our reagent solutions were stripped of metals ions, we consider the dominant oxidation route to be through the sulfur-based oxoacids which progressively proceed through the sulfenic, sulfinic, and sulfonic acids before oxidative saturation of the sulfur center to eliminate sulfate. The following four reactions, $4.4-4.7$, will initiate the oxidation of TMTU:

$$
\begin{aligned}
& \mathrm{TMTU}+\mathrm{ClO}_{2}{ }^{-} \rightarrow \mathrm{TMTUSOH}^{\mathrm{T}}+\mathrm{OCl}^{-} ; k_{1} \\
& \mathrm{TMTU}+\mathrm{HClO}_{2} \rightarrow \mathrm{TMTUSOH}+\mathrm{HOCl} ; k_{2}
\end{aligned}
$$


Low acid concentrations in figures $4.4 \mathrm{a}, 4.6 \mathrm{~b}$, and $4.6 \mathrm{c}$ will be dominated by reactions 4.4 and 4.5 , while high acid concentrations in figures $4.4 \mathrm{~b}, 4.6 \mathrm{a}$, and $4.6 \mathrm{c}$ will be dominated by reactions 4.5 and 4.7 . It will be difficult to find a balance where reaction 4.6 would contribute substantially, and even if this region is found, it should exist over a very narrow $\mathrm{pH}$ range. By utilizing the standard mass balance equations with respect to $\mathrm{Cl}(\mathrm{III})$ species and TMTU species, the rate laws for the consumption of TMTU in figures $4.6 \mathrm{a}-\mathrm{c}$ can be derived.

$$
\begin{gathered}
{[\mathrm{Cl}(\mathrm{III})]_{\mathrm{T}}=\left[\mathrm{ClO}_{2}^{-}\right]+\left[\mathrm{HClO}_{2}\right]} \\
{\left[\mathrm{TMTU}_{\mathrm{T}}=[\mathrm{TMTU}]+[\mathrm{TMTU}-\mathrm{H}]^{+}\right.}
\end{gathered}
$$

$[\mathrm{Cl}(\mathrm{III})]_{\mathrm{T}}$ and $[\mathrm{TMTU}]_{\mathrm{T}}$ are the amounts of chlorite and TMTU added to the reaction solution before they partition into the protonated and unprotonated versions. At low acid environments, the rate of consumption of TMTU will follow the following rate law:

$$
\frac{-d[\mathrm{TMTU}]}{d t}=\frac{[\mathrm{TMTU}]_{0}[\mathrm{Cl}(\mathrm{III})]_{\mathrm{T}}}{1+K_{\mathrm{al}}\left[\mathrm{H}^{+}\right]}\left(k_{1}+k_{2} K_{\mathrm{al}}\left[\mathrm{H}^{+}\right]\right)
$$

We should then expect, in high acid environments, the following complex rate law in acid:

$$
\frac{-d[\mathrm{TMTU}]}{d t}=\frac{K_{\mathrm{al}}\left[\mathrm{H}^{+}\right][\mathrm{Cl}(\mathrm{III})]_{\mathrm{T}}[\mathrm{TMTU}]_{\mathrm{T}}}{\left(1+K_{\mathrm{al}}\left[\mathrm{H}^{+}\right]\right)\left(1+K_{\mathrm{b}}\left[\mathrm{H}^{+}\right]\right)}\left(k_{2}+k_{4} K_{b}\left[H^{+}\right]\right)
$$


The derivation of equation 4.12 assumes that reactions 4.4 and 4.6 are negligible. In either case, one expects first order kinetics in chlorite and in TMTU. This is in agreement with our experimental data. The next step is to rationalize the observed change in the consumption dynamics of TMTU as acid is increased. In low acid environments, convex sigmoidal consumption profiles are observed as shown in figure $4.6 \mathrm{~b}$ and $4.6 \mathrm{c}$, while in high acid environments the consumption profile approaches a normal concave shape where standard mass action kinetics dominate (figure 4.6a and 4.6c). Autocatalysis in chlorite oxidations through $\mathrm{HOCl}$ has been reported repeatedly, and one expects this to be the case at low acid environments. All the oxidation pathways in $4.4-4.7$ produce $\mathrm{HOCl}$ as a reactive and active intermediate. $\mathrm{HOCl}$ is a much more efficient and effective oxidant than chlorite. The remaining chlorite in the reaction medium reacts with $\mathrm{HOCl}$ to produce the asymmetric intermediate which was isotopically deduced by Taube and Dodgen $[25,26]$ :

$$
\mathrm{ClO}_{2}{ }^{-}+\mathrm{HOCl}+\mathrm{H}^{+} \rightleftharpoons \mathrm{Cl}_{2} \mathrm{O}_{2}+\mathrm{H}_{2} \mathrm{O}
$$

Any further reduction of this intermediate will produce two molecules of $\mathrm{HOCl}$, thus installing quadratic autocatalysis on the rate of consumption of TMTU $[27,28,29]$.

$$
\mathrm{Cl}_{2} \mathrm{O}_{2}+2 \mathrm{H}^{+}+2 \mathrm{e}^{-} \rightarrow 2 \mathrm{HOCl}
$$

The two electron reductant could be TMTU or any of its metabolites that have not yet been oxidatively saturated to sulfate. Under these conditions that support autocatalysis, 
both $\mathrm{HClO}_{2}$ and $\mathrm{ClO}_{2}{ }^{-}$are active, with the major autocatalytic route coming from $\mathrm{ClO}_{2}{ }^{-}$. The protonated chlorous acid will not rapidly undergo reaction 4.13 which should be a composite two-step process in which chlorite initially reacts with $\mathrm{HOCl}$ followed by $\mathrm{H}^{+}$ to release a water molecule. In high acid environments, however, the predominant species is $\mathrm{HClO}_{2}$. The initial reagent ratios used in figure 4.6a have an overwhelming excess of $\mathrm{HClO}_{2}$ (ratio, $\mathrm{R}=7$ ). This approaches pseudo-first oder kinetics in which TMTU will be rapidly consumed before nonlinear behavior is allowed to assert itself. By assuming predominance of $\mathrm{HClO}_{2}$, one can re-write equation 4.12 as:

$$
\frac{-d[\mathrm{TMTU}]}{d t}=\frac{\left[\mathrm{HClO}_{2}\right]\left[\mathrm{TMTU}_{\mathrm{T}}\right.}{1+K_{\mathrm{b}}\left[\mathrm{H}^{+}\right]}\left(k_{2}+k_{4} K_{\mathrm{b}}\left[\mathrm{H}^{+}\right]\right.
$$

If $K_{\mathrm{b}}$ is small, and we assume $\left[\mathrm{HClO}_{2}\right]>>\left[\mathrm{ClO}_{2}^{-}\right]$, then equation 4.15 simplifies to:

$$
\frac{-d[\mathrm{TMTU}]}{d t}=k_{2}[\mathrm{Cl}(\mathrm{III})]_{\mathrm{T}}[\mathrm{TMTU}]_{\mathrm{T}}
$$

where a reasonable value of $k_{2}$ can be evaluated in high acid environments, for example using the first phase of the reaction in figure 4.6a. Equation 4.15 clearly supports a standard concave shape with respect to consumption of TMTU. The specific acid dependence effect will be derived from the relative values of $\left[\mathrm{H}^{+}\right], k_{4}$ and $K_{\mathrm{b}}$. However, for as long as $k_{4}>k_{2}$, acid will not be inhibitory. Acid would have been inhibitory if reaction 4.6 was the major route of oxidation of TMTU. The removal of the effectiveness of reaction 4.4 by increasing acidic conditions will bring out a visible change in the observed profile of the rate of consumption of TMTU as has been observed in figures 
4.6a, 4.6b, and 4.6c. Experimentally, the catalytic effect of acid quickly saturates and subsequently inhibits in environments of acid concentrations greater that $0.6 \mathrm{M}$ which implies that under these conditions only reaction 4.7 will be viable as reaction 4.5 loses its dominance.

\subsubsection{Further oxidation of TMTU}

The sulfenic acid formed in the rate-determining steps $4.4-4.7$, should be unstable and easily further oxidized to the more stable sulfinic and sulfonic acids in a series of irreversible, entropy driven two-electron transfer steps

$$
\begin{aligned}
& \text { TMTUSOH }+\mathrm{HOCl} \rightarrow \text { TMTUSO }_{2} \mathrm{H}+\mathrm{H}^{+}+\mathrm{Cl}^{-} \\
& \text {TMTUSO}_{2} \mathrm{H}+\mathrm{HOCl} \rightarrow \text { TMTUSO}_{3} \mathrm{H}+\mathrm{H}^{+}+\mathrm{Cl}^{-}
\end{aligned}
$$

In the absence of further oxidant, sulfenic acids are known to either dimerize through a condensation reaction with an unoxidized TMTU molecule, or disproportionate into thiosulfinates [30,31,32].

\subsubsection{Formation of products}

Current and previous studies have shown us that sulfonic acids, such as $\mathrm{TMTUSO}_{3} \mathrm{H}$, produced in reaction 4.18 are extremely stable and unreactive [33-35]. The major route for the oxidation of sulfinic acids is through cleavage of the $\mathrm{C}-\mathrm{S}$ bond to release a highly labile and reducing species, $\mathrm{HSO}_{2}{ }^{-}$and the urea analogue of the thiourea compound (see reaction 4.19) $[36,37]$. The direct oxidation of the sulfinic acid to the 
sulfonic acid produces an inert intermediate that reacts only very slowly [35]. This stability has been reported, observed, and documented in atmospheric chemistry where hydroxymethanesulfonic acid (bisulfite addition compound) is so stable that it is considered the sink for all anthropogenic sulfur dioxide emissions [38-40]. Highly acidic environments, however, stabilize the aqueous form of the sulfonic acid much more than the sulfinic acid. The cleavage of the $\mathrm{C}-\mathrm{S}$ bond in sulfinic acid is aided by the nucleophilic solvent molecules, water in this case, as shown in reaction 4.19. The same applies for sulfonic acid, 4.20. Reactions 4.19 and 4.20 are extremely rapid and facile in basic environments, and are the most important steps in guanidylation [41].

$$
\begin{aligned}
& \mathrm{Me}_{2} \mathrm{~N}(=\mathrm{NMe}) \mathrm{C}-\mathrm{SO}_{2} \mathrm{H}+\mathrm{Nu}:\left(\mathrm{H}_{2} \mathrm{O}\right) \rightarrow \mathrm{Me}_{2} \mathrm{~N}(\mathrm{NHMe}) \mathrm{C}=\mathrm{O}+\mathrm{HSO}_{2}{ }^{-}+\mathrm{H}^{+} \\
& \mathrm{Me}_{2} \mathrm{~N}(=\mathrm{NMe}) \mathrm{C}-\mathrm{SO}_{3} \mathrm{H}+\mathrm{Nu}:\left(\mathrm{H}_{2} \mathrm{O}\right) \rightarrow \mathrm{Me}_{2} \mathrm{~N}(\mathrm{NHMe}) \mathrm{C}=\mathrm{O}+\mathrm{HSO}_{3}{ }^{-}+\mathrm{H}^{+}
\end{aligned}
$$

The sulfoxylate anion and the bisulfite are rapidly oxidized to sulfate, leaving the rate of hydrolysis of the oxo-acids (4.19 and 4.20) as the rate-determining steps in the oxidation of sulfinic and sulfonic acids.

The zwitterionic forms of the sulfinic and sulfonic acids are known and shown below: 

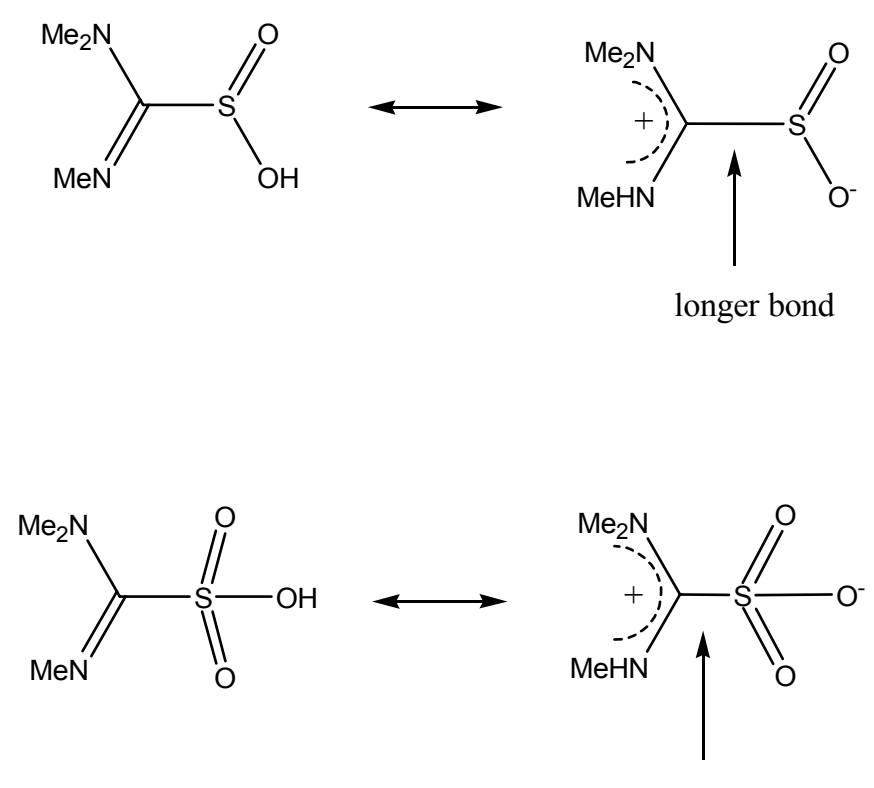

Shorter bond

The zwitterionic structures above have been proved in dimethylthiourea by the fact that in both sulfinic and sulfonic acids, the $\mathrm{C}-\mathrm{N}$ bond lengths are equivalent (1.303 $\AA$ ) and lie in between the double bond and single bond lengths. All $\mathrm{S}-\mathrm{O}$ bonds are also equivalent. Our previous studies have shown that the $\mathrm{C}-\mathrm{S}$ bond in the zwitterionic thiourea dioxide (sulfinic acid) is much longer $(1.880 \AA)$ than the one expected from merely adding covalent radii of carbon and sulfur (1.79 $\AA$ ); and that it is also longer than in the trioxide analogue $(1.815 \AA)$ [33]. Thus cleavage of the $\mathrm{C}-\mathrm{S}$ bond is much easier at the sulfinic acid stage rather than at the sulfonic acid stage. In fact, most sulfonic acids are extremely inert in medium to high acid environments [34]. The stability of the sulfonic acid at low $\mathrm{pH}$ can help explain the observed retardation in chlorine dioxide formation with acid shown in figure $4.4 \mathrm{~b}$ and the catalytic effect of low acid concentrations on the second stage of the reaction shown in figure 4.6c. As has been quantified earlier, formation of chlorine dioxide is dependent upon the formation of $\mathrm{HOCl}$; which in turn is dependent upon the rate of oxidation of the reducing substrates. The formation of a stable sulfonic acid in reaction 4.18 effectively retards further oxidation and production of $\mathrm{HOCl}$ and 
thus starving the reaction that forms chlorine dioxide. Reactions 4.19 and 4.20 produce the readily-oxidized sulfur species which increases the rate of formation of $\mathrm{HOCl}$ from chlorite. (Even though $\mathrm{HOCl}$ reacts faster with $\mathrm{HSO}_{2}^{-}$and $\mathrm{HSO}_{3}^{-}$, the most abundant species in the reaction medium is chlorite, and it is mostly through chlorite that these species will be oxidized.) The sequence of reaction steps after the formation of the sulfinic acid can be best represented by the schematic diagram shown below.

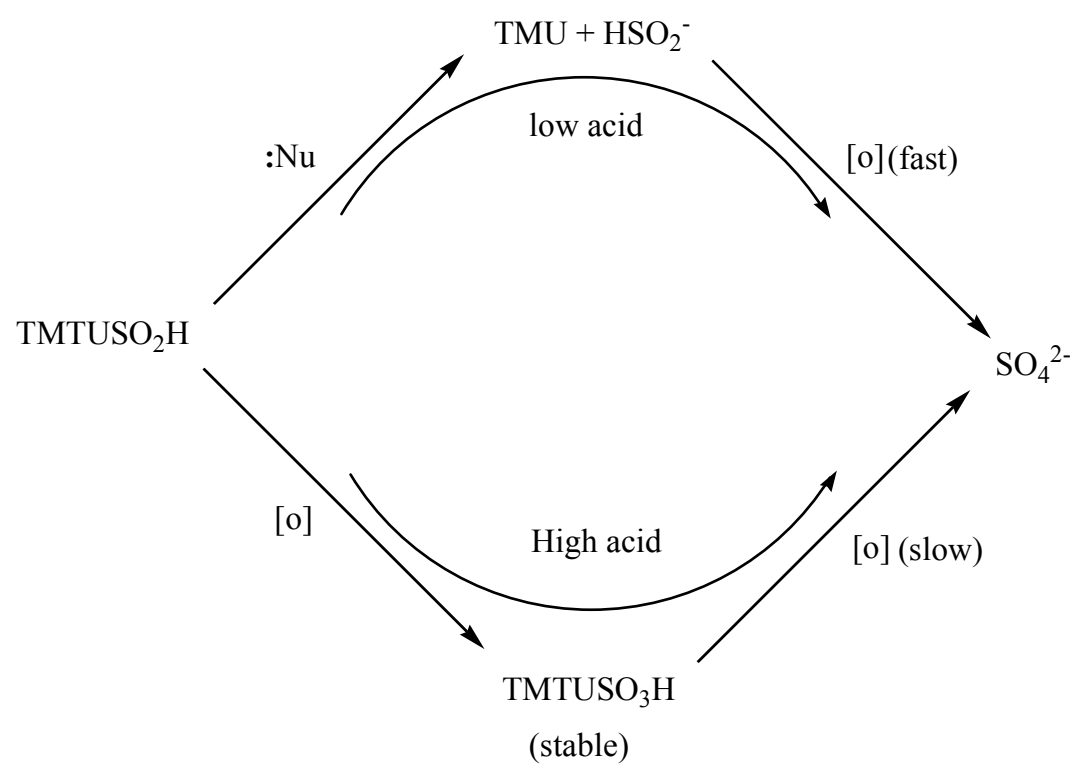

Further reaction of $\mathrm{TMTUSO}_{3} \mathrm{H}$ is very slow and it first undergoes hydrolysis to produce urea and bisulfite (reaction 4.20) which is then rapidly oxidized to sulfate [34,35]. The hydrolysis equilibrium, however, favors the sulfonic acid in high acid environments, and hence the reaction is faster if the reaction solutions have been aged before oxidation is observed [34]. The reaction's duration is roughly $200 \mathrm{~s}$; which is insufficient time for incubation of the solution to release bisulfite $[34,33]$. 


\subsubsection{Formation of chlorine dioxide}

Chlorine dioxide is primarily formed from the oxidation of chlorite by $\mathrm{HOCl}$, and to some extent aqueous chlorine. No chlorine dioxide is formed in the absence of sufficient chlorite, i.e. at the point where reactions $4.4-4.8$ are producing $\mathrm{HOCl}$. After reaction 4.13 , chlorite will be rapidly oxidized by $\mathrm{Cl}_{2} \mathrm{O}_{2}$ to form chlorine dioxide:

$$
\mathrm{Cl}_{2} \mathrm{O}_{2}+\mathrm{ClO}_{2}^{-} \rightarrow 2 \mathrm{ClO}_{2}+\mathrm{Cl}^{-}
$$

Chlorine can be formed by its reverse disproportionation reaction [42]:

$$
\mathrm{HOCl}+\mathrm{H}^{+}+\mathrm{Cl}^{-} \rightleftharpoons \mathrm{Cl}_{2}+\mathrm{H}_{2} \mathrm{O}
$$

Chlorine can also rapidly oxidize chlorite to form chlorine dioxide:

$$
\mathrm{Cl}_{2}+2 \mathrm{ClO}_{2}^{-} \rightarrow 2 \mathrm{ClO}_{2}+2 \mathrm{Cl}^{-}
$$

Since both reactions 4.21 and 4.23 are fast, the rate of formation of chlorine dioxide (before factoring in its consumption) should be proportional to the rate of formation of $\mathrm{HOCl}$ (see above). The data in figure 4.3a are easily justified on that premise: higher chlorite concentrations, through pure mass-action kinetics, will produce $\mathrm{HOCl}$ at a higher rate, thus shortening the induction period, and increasing the rate of formation of $\mathrm{HOCl}$ after the induction period. An examination of the data in figures $4.4 \mathrm{~b}$ and 4.6a will show that, even though the rate and amount of chlorine dioxide formed is 
depressed by higher acid concentrations, the rate of consumption of TMTU is still catalyzed by acid in the initial stages but decelerated later on in the reaction (examine traces a and b). The deceleration is due to the stability of the sulfonic acid in highly acidic environments such that its further oxidation is retarded by acid, resulting in a slowing down of the reaction after formation of the sulfinic and sulfonic acids (see figure 4.6c). Figure $4.7 \mathrm{~b}$ is very important in estimating the position in time at which chlorine dioxide is formed. The total consumption of TMTU and its metabolites is not necessary for the formation of chlorine dioxide as has been observed in a comparable study of the oxidation of thiourea by chlorite [16].

\subsubsection{Chlorine dioxide consumption}

Data in figures $4.9-4.11$ show typical consumption kinetics of chlorine dioxide. In all these experiments, initial concentrations of chlorine dioxide and TMTU are approximately equal. This enables features such as autocatalysis and autoinhibition to

prevail, should they exist, instead of being masked by pseudo-first order kinetics if $[\mathrm{TMTU}]_{0} \gg>\left[\mathrm{ClO}_{2}\right]_{0}$. The rapidly-increasing rate of oxidation of chlorine dioxide implicates $\mathrm{HOCl}$ autocatalysis. Oxidation by chlorine dioxide is expected to proceed through chlorite, which will then unleash the well-known oxychlorine species, HOCl. Chlorine dioxide is a radical species and should oxidize by a one-electron process. Mechanistically, we expect the electron-rich sulfur center of the TMTU to attack the electron-poor chlorine center to form a loose adduct.

$$
\mathrm{Me}_{2} \mathrm{~N}(\mathrm{NHMe}) \mathrm{C}=\mathrm{S}+\cdot \mathrm{ClO}_{2} \rightleftharpoons\left[\mathrm{Me}_{2} \mathrm{~N}(\mathrm{NHMe}) \mathrm{C}=\mathrm{S}-\mathrm{ClO}_{2}\right]
$$




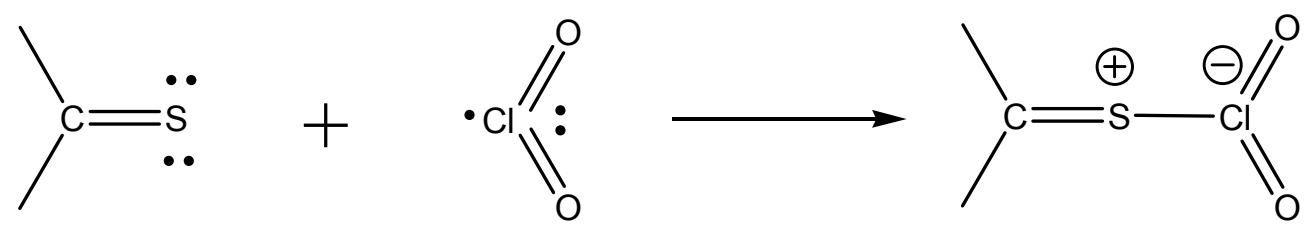

Further reaction of this adduct with another chlorine dioxide molecule will produce the sulfenic acid and chlorous acid.

$$
\left[\mathrm{Me}_{2} \mathrm{~N}(\mathrm{NHMe}) \mathrm{C}=\mathrm{S}-\mathrm{ClO}_{2}\right]+\mathrm{ClO}_{2}+\mathrm{H}_{2} \mathrm{O} \rightarrow \mathrm{TMTUSOH}+2 \mathrm{ClO}_{2}{ }^{-}+2 \mathrm{H}^{+}
$$

Each TMTU molecule will produce, in the initial stages, two molecules of chlorite, which is a better and more labile oxidizing agent than chlorine dioxide. Coupled with the acknowledged $\mathrm{HOCl}$ autocatalysis (4.13 and 4.14), we expect an ever-increasing rate of reaction. Acid concentrations inhibit the reaction (see figure 4.10) by shifting the equilibrium of the reaction 4.24 to the left due to protonation of TMTU and by the domination of $\mathrm{HClO}_{2}$ over $\mathrm{ClO}_{2}{ }^{-}$among the products formed in reaction 4.25. Figure 4.11a shows that doping the reaction mixture with micromolar concentrations of chlorite rapidly accelerates the reaction, giving credence to the fact that reduction of chlorine dioxide goes via chlorite.

\subsubsection{Experimental evaluation of some rate constants}

One can isolate one reaction from the rest of the four possible rate-determining steps $4.4-4.7$. Figure 4.7 a represents a present example of this isolation in the first stage of the reaction. One specific experimental run was performed at $1.00 \mathrm{M}$ acid where we 
assumed dominance of reaction 4.5 and $k_{2}$ was evaluated on the basis of rate equation 4.16. For statistical analysis and generation of error bars, other plots of initial rates $v s$. chlorite were made and several values of $k_{2}$ were evaluated. The compilation gave a value of $k_{2}=1.58 \pm 0.3 \times 10^{2} \mathrm{M}^{-1} \mathrm{~s}^{-1}$. Due to the approximation that most TMTU is unprotonated, this value of $k_{2}$ obtained represents an upper limit rate constant. The upper limit value of $k_{2}$ can then be used to derive $k_{1}$ (the reaction between chlorite and TMTU; reaction 4.4). Data in figure 4.6a was then used along with the rate equation 4.11 to derive a lower limit rate constant for $k_{1}$. This treatment gave a value of $k_{1}=52 \pm 9 \mathrm{M}^{-1} \mathrm{~s}^{-1}$. The inaccuracy observed in $k_{1}$ is derived from the change in kinetics as acid concentrations are increased. Acid dependence data from figure 4.6b, with its sigmoidal decay kinetics are inadequate for initial rate determinations, and very high acid concentrations gave saturation in rate with respect to acid concentrations. There was thus a very small window in which the rate equation 4.11 was applicable. Figure $4.12 \mathrm{a}$ is another special figure produced just for the generation of a specific rate constant. By varying chlorine dioxide concentrations at constant acid and TMTU concentrations, a linear relationship is obtained from where the earlier-reported bimolecular rate constant of $16 \pm 3 \mathrm{M}^{-1} \mathrm{~s}^{-1}$ was derived. In figure $4.12 \mathrm{~b}$ the acid strength was increased to $1.00 \mathrm{M}$ which gave predominantly, $\mathrm{HClO}_{2}$ as the $\mathrm{Cl}(\mathrm{III})$ species, and the protonated thiocarbamide. Under such extreme conditions, pseudo-first order kinetics could be obtained. The apparent rate constants from the semi-log plots obtained from figure $4.12 \mathrm{~b}$ were invariant to TMTU concentrations. The deduced rate constant under these conditions was $10 \mathrm{M}^{-1} \mathrm{~s}^{-1}$. 


\subsubsection{Global reaction network}

We have devised the full reaction scheme that encompasses reactions of both chlorite and chlorine dioxide with TMTU into the mechanism given in Table 4.1.

Table 4.1: The Chlorite-Chlorine Dioxide-Trimethylthiourea Reaction Network

\begin{tabular}{|c|c|}
\hline & Reaction \\
\hline M1 & $\mathrm{ClO}_{2}^{-}+\mathrm{H}^{+} \rightleftharpoons \mathrm{HClO}_{2} ; K_{\mathrm{a} 1}^{-1}=52.48 \mathrm{M}$ \\
\hline M2 & $\mathrm{TMTU}+\mathrm{H}^{+} \rightleftharpoons \mathrm{TMTU}-\mathrm{H}^{+}$ \\
\hline M3 & $\mathrm{OCl}^{-}+\mathrm{H}^{+} \rightleftharpoons \mathrm{HOCl} ; K_{\mathrm{a} 2}^{-1}=3.1 \times 10^{7} \mathrm{M}$ \\
\hline M4 & $\mathrm{ClO}_{2}^{-}+\mathrm{TMTU} \rightarrow \mathrm{TMTUSOH}+\mathrm{OCl}^{-} ; k_{1}=52 \mathrm{M}^{-1} \mathrm{~s}^{-1}$ \\
\hline M5 & $\mathrm{HClO}_{2}+\mathrm{TMTU} \rightarrow \mathrm{TMTUSOH}+\mathrm{HOCl} ; k_{2}=1.58 \times 10^{2} \mathrm{M}^{-1} \mathrm{~s}^{-1}$ \\
\hline M6 & $\mathrm{TMTU}_{-} \mathrm{H}^{+}+\mathrm{HClO}_{2} \rightarrow \mathrm{TMTUSOH}+\mathrm{HOCl}+\mathrm{H}^{+}$ \\
\hline M7 & $\mathrm{TMTUSOH}+\mathrm{HOCl} \rightarrow \mathrm{TMTUSO}_{2} \mathrm{H}+\mathrm{Cl}^{-}+\mathrm{H}^{+}$ \\
\hline M8 & TMTUSO $_{2} \mathrm{H}+\mathrm{HOCl} \rightarrow$ TMTUSO$_{3} \mathrm{H}+\mathrm{Cl}^{-}+\mathrm{H}^{+}$ \\
\hline M9 & TMTUSO $_{3} \mathrm{H}+\mathrm{HOCl}+\mathrm{H}_{2} \mathrm{O} \rightarrow \mathrm{TMU}+\mathrm{SO}_{4}{ }^{2-}+\mathrm{Cl}^{-}+3 \mathrm{H}^{+}$ \\
\hline M10 & $\mathrm{TMTU}+\mathrm{HOCl} \rightarrow \mathrm{TMTUSOH}+\mathrm{Cl}^{-}+\mathrm{H}^{+}$ \\
\hline M11 & TMTUSOH $+\mathrm{ClO}_{2}^{-} \rightarrow \mathrm{TMTUSO}_{2} \mathrm{H}+\mathrm{OCl}^{-}$ \\
\hline M12 & $\mathrm{TMTUSO}_{2} \mathrm{H}+\mathrm{ClO}_{2}^{-} \rightarrow \mathrm{TMTUSO}_{3} \mathrm{H}+\mathrm{OCl}^{-}$ \\
\hline M13 & TMTUSO $3 \mathrm{H}+\mathrm{ClO}_{2}^{-}+\mathrm{H}_{2} \mathrm{O} \rightarrow \mathrm{TMU}+\mathrm{SO}_{4}{ }^{2-}+\mathrm{HOCl}+\mathrm{H}^{+}$ \\
\hline M14 & $\mathrm{TMTUSOH}+\mathrm{HClO}_{2} \rightarrow \mathrm{TMTUSO}_{2} \mathrm{H}+\mathrm{HOCl}$ \\
\hline M15 & $\mathrm{TMTUSO}_{2} \mathrm{H}+\mathrm{HClO}_{2} \rightarrow \mathrm{TMTUSO}_{3} \mathrm{H}+\mathrm{HOCl}$ \\
\hline M16 & $\mathrm{TMTUSO}_{3} \mathrm{H}+\mathrm{HClO}_{2}+\mathrm{H}_{2} \mathrm{O} \rightarrow \mathrm{TMU}+\mathrm{SO}_{4}{ }^{2-}+\mathrm{HOCl}+2 \mathrm{H}^{+}$ \\
\hline M17 & $\mathrm{TMTUSO}_{2} \mathrm{H}+\mathrm{H}_{2} \mathrm{O} \rightleftharpoons \mathrm{TMU}+\mathrm{HSO}_{2}^{-}+\mathrm{H}^{+}$ \\
\hline M18 & $\mathrm{TMTUSO}_{3} \mathrm{H}+\mathrm{H}_{2} \mathrm{O} \rightleftharpoons \mathrm{TMU}+\mathrm{HSO}_{3}^{-}+\mathrm{H}^{+}$ \\
\hline M19 & $\mathrm{HSO}_{2}^{-}+\mathrm{HOCl} \rightarrow \mathrm{HSO}_{3}^{-}+\mathrm{Cl}^{-}+\mathrm{H}^{+}$; diffusion-controlled \\
\hline M20 & $\mathrm{HSO}_{3}^{-}+\mathrm{HOCl} \rightarrow \mathrm{SO}_{4}{ }^{2-}+\mathrm{Cl}^{-}+2 \mathrm{H}^{+}$; diffusion-controlled \\
\hline M21 & $\mathrm{ClO}_{2}^{-}+\mathrm{HOCl}+\mathrm{H}^{+} \rightleftharpoons \mathrm{Cl}_{2} \mathrm{O}_{2}+\mathrm{H}_{2} \mathrm{O} ; k_{\mathrm{f}}=1.01 \times 10^{6} \mathrm{M}^{-2} \mathrm{~s}^{-1}$ \\
\hline M22 & $\mathrm{Cl}_{2} \mathrm{O}_{2}+\mathrm{ClO}_{2}^{-} \rightarrow 2 \mathrm{ClO}_{2}+\mathrm{Cl}^{-}$ \\
\hline M23 & $\mathrm{Cl}_{2} \mathrm{O}_{2}+\mathrm{TMTU}+\mathrm{H}_{2} \mathrm{O} \rightarrow$ TMTUSOH $+2 \mathrm{HOCl}$ \\
\hline M24 & $\mathrm{Cl}_{2} \mathrm{O}_{2}+\mathrm{TMTUSOH}+\mathrm{H}_{2} \mathrm{O} \rightarrow$ TMTUSO $_{2} \mathrm{H}+2 \mathrm{HOCl}$ \\
\hline M25 & $\mathrm{Cl}_{2} \mathrm{O}_{2}+\mathrm{TMTUSO}_{2} \mathrm{H}+\mathrm{H}_{2} \mathrm{O} \rightarrow$ TMTUSO$_{3} \mathrm{H}+2 \mathrm{HOCl}$ \\
\hline M26 & $2 \mathrm{ClO}_{2}+\mathrm{TMTU}+\mathrm{H}_{2} \mathrm{O} \rightarrow$ TMTUSOH $+2 \mathrm{HClO}_{2} ; k_{\mathrm{f}}=16 \mathrm{M}^{-1} \mathrm{~s}^{-1}$ \\
\hline M27 & $2 \mathrm{ClO}_{2}+\mathrm{TMTUSOH}+\mathrm{H}_{2} \mathrm{O} \rightarrow \mathrm{TMTUSO}_{2} \mathrm{H}+2 \mathrm{HClO}_{2}$ \\
\hline M28 & $2 \mathrm{ClO}_{2}+\mathrm{TMTUSO}_{2} \mathrm{H}+\mathrm{H}_{2} \mathrm{O} \rightarrow \mathrm{TMTUSO}_{3} \mathrm{H}+2 \mathrm{HClO}_{2}$ \\
\hline
\end{tabular}

Not all 28 reactions were ever used at the same time. For example, highly acidic conditions eliminated reactions, M4 and M11 - M14. The adopted reaction network has three rapid protolytic reactions, $\mathrm{M} 1-\mathrm{M} 3$, and three initiation reactions, M4 - M6. The 
fourth reaction between a protonated TMTU molecule and chlorite, reaction 4.6, was eliminated from the mechanism. $\mathrm{HOCl}$ is adopted in this mechanism as the dominant oxidizing species, reaction M7 - M10. The autocatalysis is accounted for in reactions M21, and M23 - M25.

A reasonable attempt to simulate this mechanism is heavily dependent on the knowledge of the kinetic parameters for reactions M17 and M18. There have been no studies so far on the kinetics and mechanisms of these hydrolysis reactions which appear to be generally base catalyzed. Without the requisite knowledge of these kinetic parameters, only the first phases of figure $4.6 \mathrm{a}$ and $4.7 \mathrm{a}$ could be satisfactorily simulated. The observed autocatalytic decay of the sulfonic acid (second phase of the reaction in figures 4.6a, 4.6b, and 4.7a) is derived from the expected rapid reaction between the sulfur leaving groups in reactions M17 and M18 with chlorine dioxide. Chlorine dioxide should rapidly form an adduct with bisulfite which will then react with another chlorine dioxide molecule to form chlorite.

$$
\begin{gathered}
\mathrm{ClO}_{2}+\mathrm{HSO}_{3}^{-} \rightleftharpoons\left[\mathrm{O}_{2} \mathrm{Cl}_{-}-\mathrm{SO}_{3} \mathrm{H}^{-}\right] \text {; rapid } \\
{\left[\mathrm{O}_{2} \mathrm{Cl}_{-}-\mathrm{SO}_{3} \mathrm{H}^{-}\right]+\mathrm{ClO}_{2}+\mathrm{H}_{2} \mathrm{O} \rightarrow \mathrm{SO}_{4}{ }^{2-}+2 \mathrm{ClO}_{2}^{-}+3 \mathrm{H}^{+} \text {; fast }}
\end{gathered}
$$

The rapid formation of chlorite accelerates the reaction. Adduct formation between radical $\mathrm{ClO}_{2}$ species and electron-rich nucleophiles has been recently reported by Nagypal et al on the reaction of chlorine dioxide with thiosulfate and tetrathione [43,44]. These are rapid reactions with rates of the 4.26-type reactions close to diffusion control. 
Although no similar studies have been reported with bisulfite, one would assume that 4.26 should also be extremely rapid.

\subsection{CONCLUSION}

The kinetics of the oxidation of a substituted thiourea, TMTU, by chlorite have been studied in a slightly acidic media. Our studies have shown that the oxidation kinetics of TMTU by chlorite are more complex than the comparable oxidation of the unsubstituted thiourea. TMTU reactions are much faster and possess a much more complex behavior with respect to acid. Most of the complexity lies in the reactivity of the most important metabolite of TMTU; the sulfinic acid. While it is easily oxidized to the sulfonic acid, that route quickly terminates as further reaction from that metabolite is very slow. What could be most important is the ease by which the $\mathrm{C}-\mathrm{S}$ bond is cleaved in the sulfinic acid because under aerobic conditions, we expect a concomitant production of reactive oxygen species that might bring with them inadvertent toxicity [36]. 


\section{References}

1. M. Orban, C. Dateo, P. De Kepper, and I. R. Epstein, "Systematic design of chemical oscillators. 11. Chlorite oscillators: New experimental examples, tristability, and preliminary classification," J. Am. Chem. Soc. 104, 5911-5918 (1982).

2. M. Alamgir and I. R. Epstein, "Systematic design of chemical oscillators. Part 31. New chlorite oscillators: Chlorite-bromide and chlorite-thiocyanate in a CSTR," J. Phys. Chem. 89, 3611-3614 (1985).

3. M. Orban, P. De Kepper, and I. R. Epstein, "Systematic design of chemical oscillators. Part 7. An iodine-free chlorite-based oscillator. The chlorite-thiosulfate reaction in a continuous flow stirred tank reactor," J. Phys. Chem. 86, 431-433 (1982).

4. R. H. Simoyi, J. Masere, C. Muzimbaranda, M. Manyonda, and S. Dube, "Traveling wave in the chlorite-thiourea reaction," Int. J. Chem. Kinet. 23, 419-429 (1991).

5. C. R. Chinake and R. H. Simoyi, "Fingering patterns and other interesting dynamics in the chemical waves generated by the chlorite-thiourea reaction," J. Phys. Chem. 98, 4012-4019 (1994).

6. R. H. Simoyi, M. Manyonda, J. Masere, M. Mtambo, I. Ncube, H. Patel, I. R. Epstein, and K. Kustin, "Systematic design of chemical oscillators. 66. Kinetics and mechanism of the oxidation of thiocyanate by iodate," J. Phys. Chem. 95, 770-774 (1991).

7. M. Orban and I. R. Epstein, "Systematic design of chemical oscillators. Part 13. Complex periodic and aperiodic oscillation in the chlorite-thiosulfate reaction," $\mathrm{J}$. Phys. Chem. 86, 3907-3910 (1982).

8. C. J. Doona, R. Blittersdorf, and F. W. Schneider, "Deterministic chaos arising from homoclinicity in the chlorite-thiourea oscillator," J. Phys. Chem. 97, 7258-7263 (1993).

9. B. S. Martincigh, C. R. Chinake, T. Howes, and R. H. Simoyi, "Self-organization with traveling waves: a case for a convective torus," Phys. Rev. E 55, 7299-7303 (1997).

10. B. S. Martincigh, M. J. B. Hauser, and R. H. Simoyi, "Formation of thermal plumes in an autocatalytic exothermic chemical reaction," Phys. Rev. E 52, 6146-6153 (1995).

11. B. S. Martincigh and R. H. Simoyi, "Convective instabilities induced by an exothermic autocatalytic chemical reaction," Phys. Rev. E 52, 1606-1613 (1995). 
12. I. R. Wilson and G. M. Harris, "The oxidation of thiocyanate ion by hydrogen peroxide. I. The pH-independent reaction," J. Am. Chem. Soc. 82, 4515-4517 (1960).

13. I. R. Wilson and G. M. Harris, "The oxidation of thiocyanate ion by hydrogen peroxide. II. The acid-catalyzed reaction," J. Am. Chem. Soc. 83, 286-289 (1961).

14. R. H. Simoyi, "New bromate oscillator: The bromate-thiourea reaction in a CSTR," J. Phys. Chem. 90, 2802-2804 (1986).

15. R. H. Simoyi and I. R. Epstein, "Systematic design of chemical oscillators. 40. Oxidation of thiourea by aqueous bromine: autocatalysis by bromide," J. Phys. Chem. 91, 5124-5128 (1987).

16. I. R. Epstein, K. Kustin, and R. H. Simoyi, "Systematic design of chemical oscillators. 78. Kinetics and mechanism of the chlorite-thiourea reaction in acidic medium," J. Phys. Chem. 96, 5852-5856 (1992).

17. S. B. Jonnalagadda, C. R. Chinake, and R. H. Simoyi, "Oxyhalogen-sulfur chemistry: Bromate Oxidation of 1-methyl-2-thiourea in acidic medium," J. Phys. Chem. 100, 13521-13530 (1996).

18. C. R. Elcombe, J. Odum, J. R. Foster, S. Stone, S. Hasmall, A. R. Soames, I. Kimber, and J. Ashby, "Prediction of rodent nongenotoxic carcinogenesis: Evaluation of biochemical and tissue changes in rodents following exposure to nine nongenotoxic NTP carcinogens," Environ. Health Perspect. 110, 363-375 (2002).

19. E. Dybing, T. Sanner, H. Roelfzema, D. Kroese, and R. W. Tennant, "T25: a simplified carcinogenic potency index: description of the system and study of correlations between carcinogenic potency and species/site specificity and mutagenicity," Pharmacol. Toxicol. 80, 272-279 (1997).

20. J. Darkwa, R. Olojo, E. Chikwana, and R. H. Simoyi, "Antioxidant chemistry: oxidation of L-Cysteine and its metabolites by chlorite and chlorine dioxide," J. Phys. Chem. A 108, 5576-5587 (2004).

21. D. M. Kern and C. H. Kim, "Iodine catalysis in the chlorite-iodide reaction," J. Am. Chem. Soc. 87, 5309-5313 (1965).

22. A. Indelli, "Kinetic study on the reaction of sodium chlorite with potassium iodide," J. Phys. Chem. 68, 3027-3031 (1964).

23. L. Pecci, G. Montefoschi, G. Musci, and D. Cavallini, "Novel findings on the copper-catalyzed oxidation of cysteine," Amino Acids 13, 355-367 (1997).

24. T. M. Jeitner and D. A. Lawrence, "Mechanisms for the cytotoxicity of cysteamine," Toxicol. Sci. 63, 57-64 (2001). 
25. H. Dodgen and H. Taube, "Exchange of chlorine dioxide with chlorite ion and with chlorine in other oxidation states," J. Am. Chem. Soc. 71, 2501-2504 (1949).

26. H. Taube and H. Dodgen, "Applications of radioactive chlorine to the study of the mechanisms of reactions involving changes in the oxidation state of chlorine," $\mathrm{J}$. Am. Chem. Soc. 71, 3330-3336 (1949).

27. G. Rabai and M. Orban, "General model for the chlorite ion based chemical oscillators," J. Phys. Chem. 97, 5935-5939 (1993).

28. G. Peintler, I. Nagypal, and I. R. Epstein, "Systematic design of chemical oscillators. 60. Kinetics and mechanism of the reaction between chlorite ion and hypochlorous acid," J. Phys. Chem. 94, 2954-2958 (1990).

29. M. A. Salem, C. R. Chinake, and R. H. Simoyi, "Nonlinear dynamics in chemistry derived from sulfur chemistry. Part 13. Oxyhalogen-sulfur chemistry: Oxidation of hydroxymethanesulfinic acid by chlorite," J. Phys. Chem. 100, 9377-9384 (1996).

30. G. Capozzi and G. Modena, "Oxidation of thiols," in The Chemistry of the Thiol Group: Part 2, edited by S.Patai, (J. Wiley and Sons, New York, 1974) p. 785-839.

31. J. Choi and N. M. Yoon, "Synthesis of Disulfides by Copper-Catalyzed Disproportionation of Thiols," J. Org. Chem. 60, 3266-3267 (1995).

32. A. McKillop, D. Koyuncu, A. Krief, W. Dumont, P. Renier, and M. Trabelsi, "Efficient, high yield oxidation of thiols and selenols to disulfides and diselenides," Tetrahedron Lett. 31, 5007-5010 (1990).

33. S. V. Makarov, C. Mundoma, J. H. Penn, J. L. Petersen, S. A. Svarovsky, and R. H. Simoyi, "Structure and stability of aminoiminomethanesulfonic acid," Inorg. Chim. Acta 286, 149-154 (1999).

34. S. V. Makarov, C. Mundoma, J. H. Penn, S. A. Svarovsky, and R. H. Simoyi, "New and surprising experimental results from the oxidation of sulfinic and sulfonic acids," J. Phys. Chem. A 102, 6786-6792 (1998).

35. J. F. Ojo, A. Otoikhian, R. Olojo, and R. H. Simoyi, "Oxyhalogen-sulfur chemistry: Nonlinear oxidation kinetics of hydroxymethanesulfinic acid by acidic iodate," J. Phys. Chem. A 108, 2457-2463 (2004).

36. S. V. Makarov, C. Mundoma, S. A. Svarovsky, X. Shi, P. M. Gannett, and R. H. Simoyi, "Reactive oxygen species in the aerobic decomposition of sodium hydroxymethanesulfinate," Arch. Biochem. Biophys. 367, 289-296 (1999).

37. S. A. Svarovsky, R. H. Simoyi, and S. V. Makarov, "Reactive oxygen species in aerobic decomposition of thiourea dioxides," J. Chem. Soc. Dalton 2000, 511-514 (2000). 
38. U. Deister, R. Neeb, G. Helas, and P. Warneck, "Temperature dependence of the equilibrium $\mathrm{CH}_{2}(\mathrm{OH})_{2}+\mathrm{HSO}_{3}{ }^{-}=\mathrm{CH}_{2}(\mathrm{OH}) \mathrm{SO}_{3}{ }^{-}+\mathrm{H}_{2} \mathrm{O}$ in aqueous solution," J. Phys. Chem. 90, 3213-3217 (1986).

39. P. Warneck, "Sulfur dioxide in rain clouds: Gas-liquid scavenging efficiencies and wet deposition rates in the presence of formaldehyde," J. Atmos. Chem. 8, 99-117 (1989).

40. Y. Zuo, "Light-induced formation of hydroxyl radicals in fog waters determined by an authentic fog constituent, hydroxymethanesulfonate," Chemosphere 51, 175-179 (2003).

41. C. A. Maryanoff, R. C. Stanzione, J. N. Plampin, and J. E. Mills, "A convenient synthesis of guanidines from thioureas," J. Org. Chem. 51, 1882-1884 (1986).

42. M. Eigen and K. Kustin, "Kinetics of halogen hydrolysis," J. Am. Chem. Soc. 84, 1355-1361 (1962).

43. A. K. Horvath, I. Nagypal, and I. R. Epstein, "Kinetics and Mechanism of the Chlorine Dioxide-Tetrathionate Reaction," J. Phys. Chem. A 107, 10063-10068 (2003).

44. A. K. Horvath and I. Nagypal, "Kinetics and Mechanism of the Reaction between Thiosulfate and Chlorine Dioxide," J. Phys. Chem. A 102, 7267-7272 (1998). 


\section{CHAPTER 5}

\section{Oxidation of Phenylthiourea by Chlorite in Acidic Media}

\subsection{INTRODUCTION}

Phenylthiourea, PTU, also known as phenylthiocarbamide is a synthetic organic molecule which is often used as an odorant and testant [1,2]. PTU has the unusual property of either tasting very bitter, or being virtually tasteless depending on the genetic makeup of the tester $[3,4]$. The ability to taste PTU is a dominant genetic trait [2]. PTU tasting is one of the most administered genetic tests in the human population. PTU, an inhibitor of dopa oxidase [5] and tyrosinase [6-8] is used in zebrafish research to suppress pigmentation in developing embryos $[9,10,7]$. Phenylthiourea contains the thiocarbamide group that is responsible for goitrogenic activity [11,12] in methimazole [13] and 6propyl-2-thiouracil [14]. PTU's use as a goitrogenic was discontinued due to its strong toxicological side effects $[15,16]$.

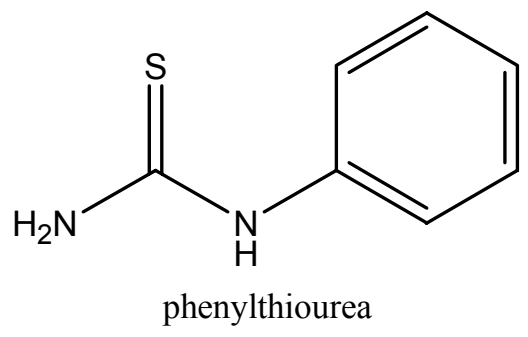

It was reported in a 1961 publication that phenylthiourea was extremely toxic to rats, but that diphenylthiourea was not [17]. There was and has never been a justification why such physiological differences exist in very similar compounds. No doubt the metabolic pathways of the two compounds must be different, but how, and why? Work 
done by Dieke et al. [18] revealed that the acute toxicity of substituted thioureas was enhanced when a single aromatic radical was attached to one of the $\mathrm{N}$ atoms. Toxicity decreased when two or more substituents were attached either on the same or on both $\mathrm{N}$ atoms. The toxicity can be narrowed to the either the parent compound, (e.g. PTU), metabolic intermediates, or the sulfur-based leaving groups. Most of the toxicological effects of organosulfur compounds are usually attributed to the S-oxygenation of the thiourea moiety of the compound.

Several theories have been postulated on the toxicities of thioureas although the mechanisms have not yet been clarified. It is generally agreed that the toxicity of thiocarbamides is related to the extent of their metabolic desulfurization, with the more highly desulfurized compounds being more toxic. For example, diphenylthiourea, which is non-toxic, is excreted primarily as the aromatic ring hydroxylated products but with the thione group intact [19]. The nature of the leaving group therefore seems to be the major source of toxicity.

Earlier studies of thiourea metabolism have shown that the major metabolite of ${ }^{35}$ S-phenylthiourea in rats was ${ }^{35} \mathrm{~S}$-inorganic sulfate. Since phenylcarbamic acid and phenylurea are also produced it was postulated that desulfurization was via a reductive mechanism with the subsequent production of hydrogen sulfide, a toxic metabolite. No studies, however, have been done to evaluate the mechanism, products, and intermediates, in the oxidation of PTU, as oxidation of this sulfur containing compound should represent an important aspect of its metabolism. The molecular basis for Soxygenation of sulfur compounds is still not well understood. This chapter will report on the results of a comprehensive study of the mechanism of the oxidation of PTU by 
chlorite. The S-oxygenation mechanism obtained should contribute greatly to the possible elucidation of the metabolic activation of PTU. Of special interest is the fate of the C-S bond after the full oxidation of PTU. Its cleavage or lack of, would give a better insight into the speculation of a possible mechanism for its toxicity.

\subsection{RESULTS}

\subsubsection{Stoichiometry}

The reaction involved successive oxidation of the sulfur center to give sulfate and a urea residue as the products:

$$
2 \mathrm{ClO}_{2}^{-}+\mathrm{PhN}(\mathrm{H}) \mathrm{CSNH}_{2}+\mathrm{H}_{2} \mathrm{O} \rightarrow \mathrm{SO}_{4}{ }^{2-}+\mathrm{PhN}(\mathrm{H}) \mathrm{CONH}_{2}+2 \mathrm{Cl}^{-}+2 \mathrm{H}^{+} \quad 5.1
$$

This stoichiometry was determined by a combination of spectrophotometric, titrimetric, and gravimetric techniques. Figure 5.1 shows superimposed UV spectra of the three major reagents in the reaction mixture; (a) phenylthiourea, PTU ( $\lambda_{\max }=249 \mathrm{~nm}, \epsilon_{\max }=$ $\left.12,312 \mathrm{M}^{-1} \mathrm{~cm}^{-1}\right)$, (b) phenylurea $\left(\lambda_{\max }=235 \mathrm{~nm}, \epsilon_{\max }=117,070 \mathrm{M}^{-1} \mathrm{~cm}^{-1}\right)$, and (c) chlorine dioxide $\left(\lambda_{\max }=360 \mathrm{~nm}, \epsilon_{\max }=1,265 \mathrm{M}^{-1} \mathrm{~cm}^{-1}\right)$. Chlorine dioxide has negligible absorbance at $249 \mathrm{~nm}$ as shown by the low absorptivity coefficient $\left(\epsilon_{249}=130 \mathrm{M}^{-1} \mathrm{~cm}^{-1}\right)$. Phenylurea on the other hand, contributes significantly to the absorbance at $249 \mathrm{~nm}$ owing to its high absorptivity coefficient. Initial rate measurements at $249 \mathrm{~nm}$ were thus only able to deliver accurate information at the beginning of the reaction before concentrations of phenylurea and chlorine dioxide had accumulated. Both phenylthiourea and phenylurea had negligible contributions to absorbance observed at $360 \mathrm{~nm}$. 


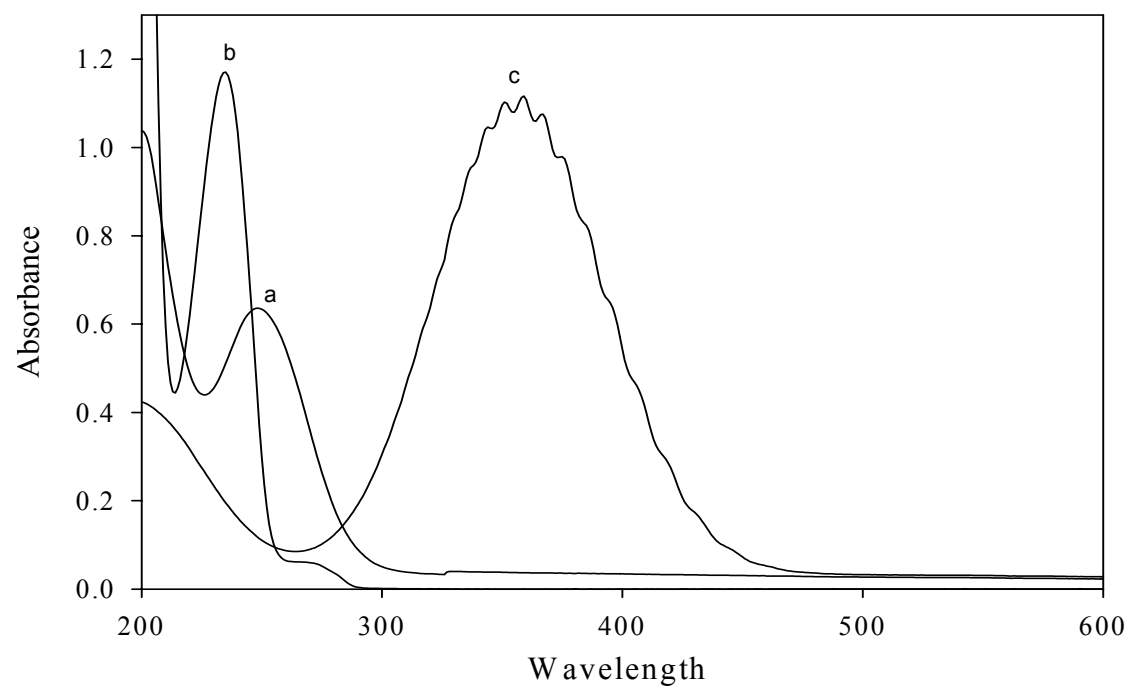

Figure 5.1a: UV absorption spectrum of (a) $5.000 \times 10^{-5} \mathrm{M}$ PTU, (b) $1.0 \times 10^{-5} \mathrm{M}$ phenlyurea, (c) $8.758 \times 10^{-4} \mathrm{M} \mathrm{ClO}_{2}$ showing peaks at $248 \mathrm{~nm}, 235 \mathrm{~nm}$ and $360 \mathrm{~nm}$ respectively. There is no interference from the substrate or product at $360 \mathrm{~nm}$.

Stoichiometry 5.1 was achieved in conditions of excess chlorite; with an oxidant to reductant ratio, $\mathrm{R}=\left[\mathrm{ClO}_{2}^{-}\right]_{0} /[\mathrm{PTU}]_{0} \geq 2.0$, and after prolonged incubation of the reaction solutions. The stoichiometry was also confirmed spectrophotometrically by observing the absorption peak of PTU at $249 \mathrm{~nm}$ and the chlorine dioxide peak at $360 \mathrm{~nm}$. Figure 5.1b shows a series of spectra taken at various ratios of chlorite to PTU. Successive increases in chlorite concentrations for a fixed amount of $[\mathrm{PTU}]_{0}$ showed a gradual decrease in the peak at $249 \mathrm{~nm}$, until it attained some baseline absorbance due to the product, phenylurea. Trace e in figure $5.1 \mathrm{~b}$ is the same as that of phenylurea (trace $\mathrm{b}$ ) in figure 5.1a. Chlorite concentrations in excess of that required by stoichiometry 5.1 gave chlorine dioxide as a product, and this could be detected by its absorption peak at 360 nm. Qualitatively, it could also be detected by the characteristic deep blue-black coloration it gave with starch prepared with mercuric iodide. Contrary to other oxyhalogen oxidations [20], the chlorine dioxide formed could not be utilized for 
stoichiometric determinations because the excess chlorite slowly decomposed in acidic media over several days to give chlorine dioxide which could not be related to the initial amount of PTU [21]:

$$
5 \mathrm{ClO}_{2}^{-}+4 \mathrm{H}^{+} \rightarrow 4 \mathrm{ClO}_{2(\mathrm{aq})}+2 \mathrm{H}_{2} \mathrm{O}+\mathrm{Cl}^{-}
$$

Iodometric techniques, however, were utilized in order to evaluate the excess oxidizing power in conditions of excess chlorite by addition of excess acidified iodide which was then titrated against standard thiosulfate with freshly-prepared starch as an indicator.

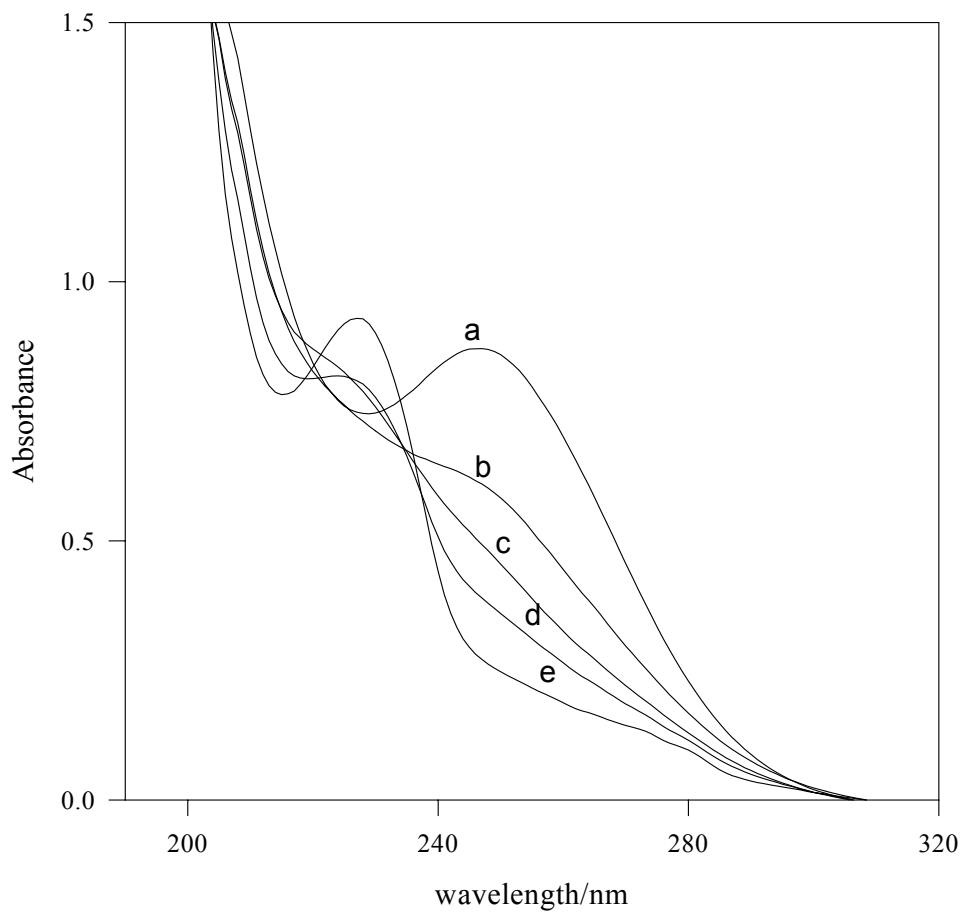

Figure 5.1b: Spectral scans of reagent solutions containing phenylthiourea and varying concentrations of chlorite between 200 and $350 \mathrm{~nm}$. PTU has a well-defined peak at $249 \mathrm{~nm}$ which progressively diminishes with increasing chlorite concentrations. At very high concentrations a peak of chlorine dioxide is observed at $360 \mathrm{~nm}$. $[\mathrm{PTU}]_{0}=5 \times 10^{-4} \mathrm{M},\left[\mathrm{H}^{+}\right]_{0}=$ $0.125 \mathrm{M},\left[\mathrm{ClO}_{2}^{-}\right]_{0}=$ (a) $2.5 \times 10^{-5} \mathrm{M}$, (b) $5.0 \times 10^{-5} \mathrm{M}$, (c) $1.0 \times 10^{-4} \mathrm{M}$, (d) $2.0 \times 10^{-4} \mathrm{M}$ and (e) $3.5 \mathrm{x}$ $10^{-4} \mathrm{M}$

A plot of thiosulfate titer volume vs. initial chlorite concentrations yielded a straight line.

Extrapolating to zero titer (chlorite concentration axis), gave an intercept where 
stoichiometry 5.1 is sufficiently satisfied, giving the exact amount of chlorite needed to just consume PTU. The analysis, gave a stoichiometric ratio slightly greater than the $2: 1$ given in reaction 5.1 especially in low acid conditions. This could be attributed to the reaction of chlorite with $\mathrm{HOCl}$ to give an inert chlorate [22,23]:

$$
\mathrm{ClO}_{2}^{-}+\mathrm{HOCl} \rightarrow \mathrm{ClO}_{3}^{-}+\mathrm{Cl}^{-}+\mathrm{H}^{+}
$$

Pathway 5.3 is insignificant in highly acidic environments where reaction 5.2 is dominant [24]. Although reaction 5.3 deactivated some oxychlorine species, reaction 5.2, however, did not alter the overall oxidizing power of the product solution and iodometric techniques could still be employed to evaluate the excess $\mathrm{ClO}_{2}^{-}$. The stoichiometry of the chlorine dioxide/PTU reaction was experimentally determined to be:

$$
8 \mathrm{ClO}_{2}+5 \mathrm{PhN}(\mathrm{H}) \mathrm{CSNH}_{2}+9 \mathrm{H}_{2} \mathrm{O} \rightarrow 5 \mathrm{SO}_{4}{ }^{2-}+5 \mathrm{PhN}(\mathrm{H}) \mathrm{CONH}_{2}+8 \mathrm{Cl}^{-}+18 \mathrm{H}^{+}
$$

This stoichiometry was derived spectrophotometrically as well as by iodometric and titrimetric techniques. Stoichiometry 5.4, however, was obtained after prolonged incubation periods of up to $48 \mathrm{~h}$.

\subsubsection{Reaction Kinetics}

\subsubsection{Chlorite Dependence}

The reaction dynamics were extremely complex, with a highly complex dependence on the $\mathrm{pH}$ of the reaction environment. The reaction dynamics were less 
complex in conditions of high excess chlorite, $\mathrm{R} \geq 4$, and in high acid concentrations, $\left[\mathrm{H}^{+}\right]_{0} \geq 0.10 \mathrm{M}$. Under these conditions the reaction showed a clean induction period before the formation of chlorine dioxide. Figure 5.2a shows such traces at $360 \mathrm{~nm}$.

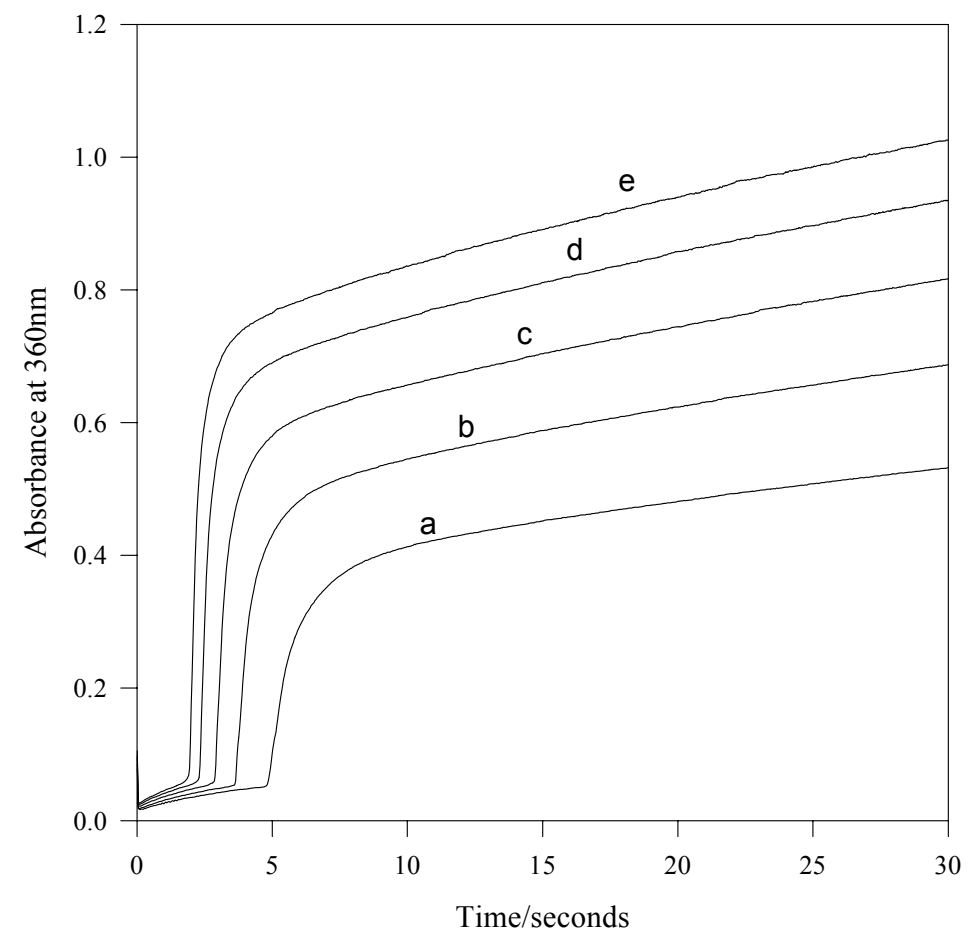

Figure 5.2a: General absorbance traces at $360 \mathrm{~nm}$ of the reaction in excess chlorite $\left(\left[\mathrm{ClO}_{2}{ }^{-}\right.\right.$ ]$\left._{0} /[\mathrm{PTU}]_{0}>4.00\right)$. The reaction starts with a short induction period followed by rapid formation of chlorine dioxide. $[\mathrm{PTU}]_{0}=1.25 \times 10^{-3} \mathrm{M},\left[\mathrm{H}^{+}\right]_{0}=0.125 \mathrm{M},\left[\mathrm{ClO}_{2}^{-}\right]_{0}=$ (a) $5 \times 10^{-3} \mathrm{M}$, (b) $6.25 \mathrm{x}$ $10^{-3} \mathrm{M}$, (c) $7.5 \times 10^{-3} \mathrm{M}$, (d) $8.75 \times 10^{-3} \mathrm{M}$ and (e) $10 \times 10^{-3} \mathrm{M}$

The rate of formation of chlorine dioxide after the induction period is directly proportional to the initial chlorite concentrations. There is an inverse relationship between the induction period and the initial chlorite concentrations (see figure 5.2b). 


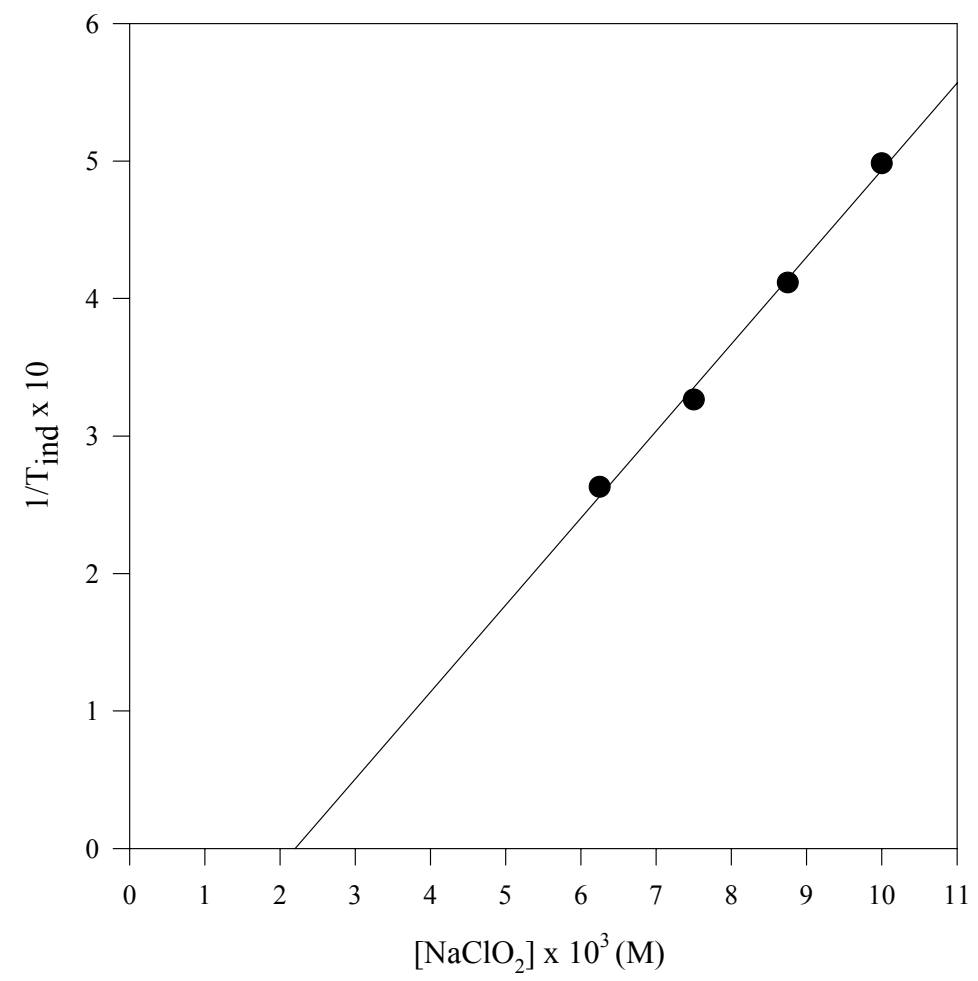

Figure 5.2b: Effect of chlorite on the induction period. An inverse relationship also exists for a long range of chlorite concentrations. $[\mathrm{PTU}]_{0}=1.25 \times 10^{-3} \mathrm{M},\left[\mathrm{H}^{+}\right]_{0}=0.125 \mathrm{M}$

This appears to indicate that chlorite is involved in the reaction kinetics that consumes PTU to the first power. The plot in figure $5.2 \mathrm{~b}$ was also utilized to confirm reaction stoichiometry 5.1. By extrapolating to $\mathrm{T}_{\mathrm{ind}}=\infty,\left(1 / \mathrm{T}_{\mathrm{Ind}}=0\right)$, one can obtain the minimum amount of $\left[\mathrm{ClO}_{2}^{-}\right]_{0}$ which gave $\mathrm{ClO}_{2}(\mathrm{aq})$ as a product, thus confirming stoichiometry 5.1. This was after assuming that no chlorine dioxide would be formed with $\left[\mathrm{ClO}_{2}^{-}\right]_{0}$ less than that required for stoichiometry 5.1. The data in figure $5.2 \mathrm{~b}$ shows that, with $[\mathrm{PTU}]_{0}=$ $0.00125 \mathrm{M}$, the threshold concentration of chlorite needed for the formation of chlorine dioxide was $0.0024 \mathrm{M}$, which is very close to the $2: 1$ ratio obtained in reaction 5.1. 


\subsubsection{Acid Dependence}

The reaction presented a very complex dependence on acid. Acid concentrations influenced the induction period, as well as the rate of formation and amount of chlorine dioxide produced after the induction period. The effect of acid also varied depending on oxidant to reductant ratios as well as the range of acid concentrations used.

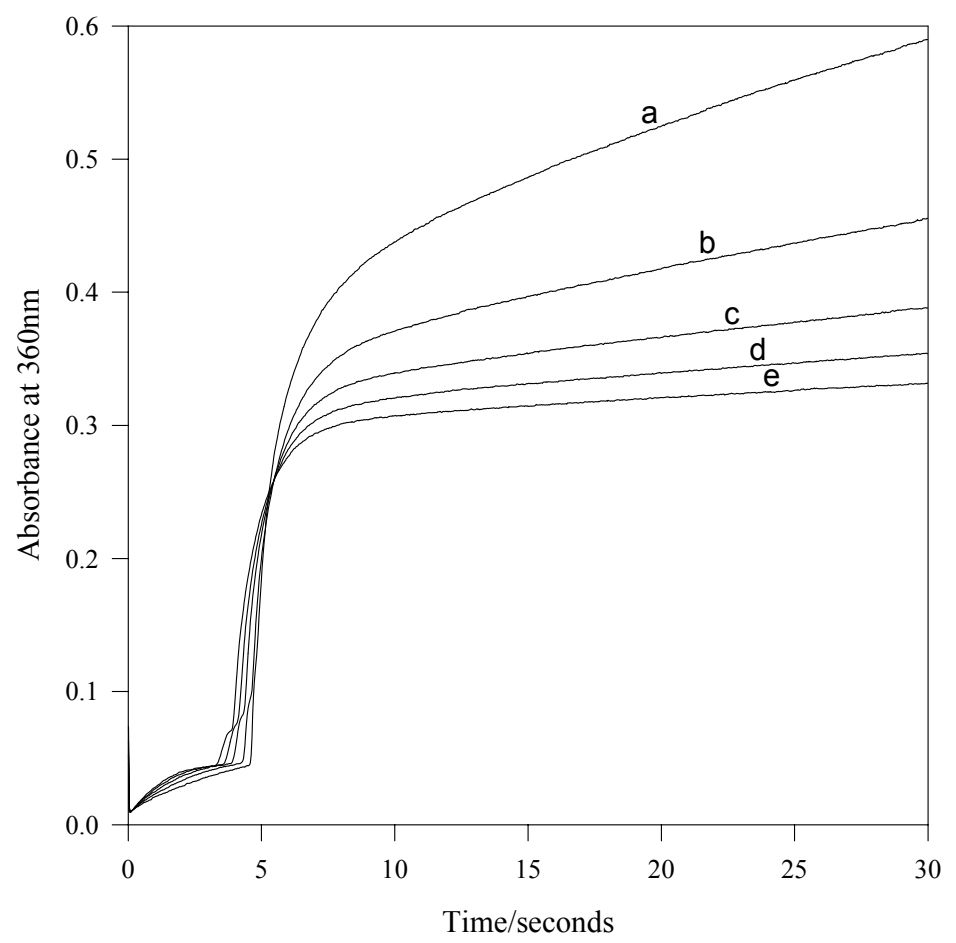

Figure 5.3a: Effect of acid concentrations on the reaction profiles with $\left[\mathrm{ClO}_{2}{ }^{-}\right]_{0} /[\mathrm{PTU}]_{0}=4.00$. The observed induction period before formation of chlorine dioxide as well as the rate of formation of chlorine dioxide become insensitive to acid at high concentrations. $[\mathrm{PTU}]_{0}=1.25 \mathrm{x}$ $10^{-3} \mathrm{M},\left[\mathrm{ClO}_{2}{ }^{-}\right]_{0}=5.0 \times 10^{-3} \mathrm{M},\left[\mathrm{H}^{+}\right]_{0}=$ (a) $0.1 \mathrm{M}$, (b) $0.2 \mathrm{M}$, (c) $0.3 \mathrm{M}$, (d) $0.4 \mathrm{M}$ and (e) $0.5 \mathrm{M}$

In highly acidic conditions, $\left[\mathrm{H}^{+}\right]_{0}>0.10 \mathrm{M}$ with $2 \leq \mathrm{R} \leq 4$, acid concentrations reduced the induction period (see figure 5.3a). This effect on the induction period is not as pronounced as that observed with chlorite (see figures 5.2a and 5.2b). These acid concentrations did not affect the rapid rate of formation of chlorine dioxide at the end of the induction period. Higher acid concentrations, however, produced lower 
concentrations of chlorine dioxide (again, see figure 5.3a). This was unexpected since it is known that chlorine dioxide formation arises from interactions among oxychlorine species which are universally accepted to be acid-catalyzed. The depressed concentrations of chlorine dioxide are only possible if subsequent reactions in the reaction mixture that consume chlorine dioxide are acid-catalyzed or if reactions that form chlorine dioxide are inhibited by acid. Surprisingly, also, lower acid concentrations $0.005 \mathrm{M}<\left[\mathrm{H}^{+}\right]<0.015 \mathrm{M}$, had no visible effect on the induction period (see figures $5.3 \mathrm{~b}$ and 5.3c). At lower ratios, $2<\mathrm{R}<4$, there was no sharp and discernible induction period, but a swift and instant formation of chlorine dioxide. At higher ratios, $R \geq 10$, the reactions also showed an invariant induction period irrespective of changes in acid concentrations (figures 5.3a and 5.3b) and in PTU concentrations.

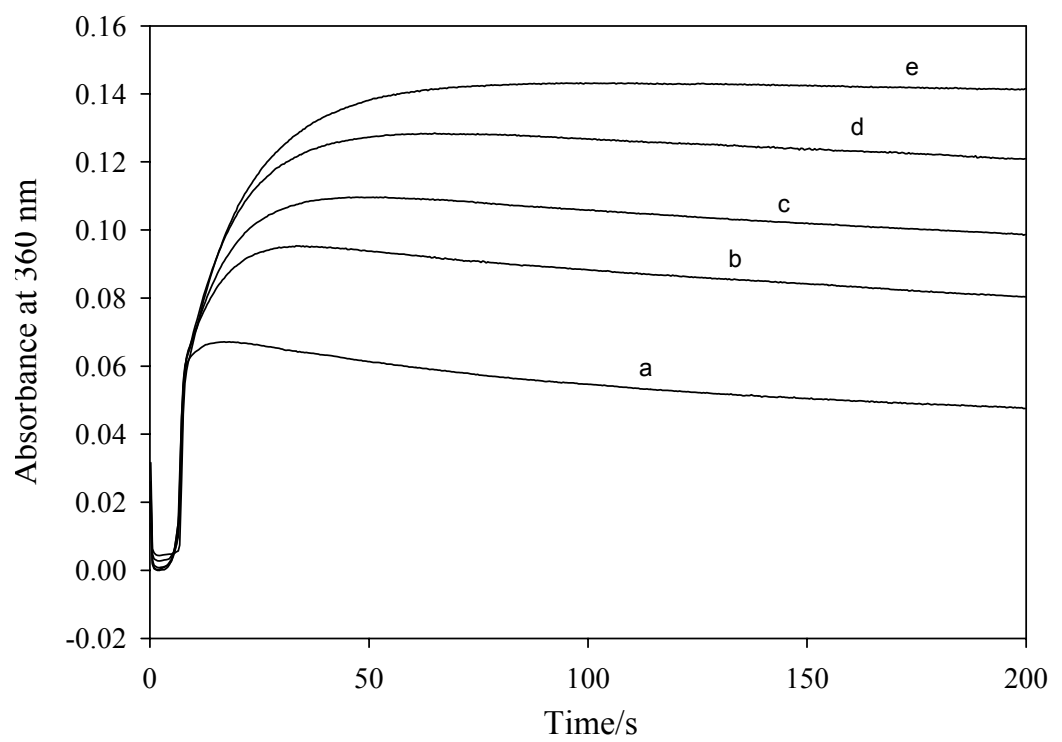

Figure 5.3b: Effect of acid at high oxidant to reductant ratios; $\left[\mathrm{ClO}_{2}^{-}\right]_{0} /[\mathrm{PTU}]_{0}=10.0$. In this case there is no well defined induction period. At low acid concentrations the rate of formation of chlorine dioxide as well as the induction period is insensitive to acid, although the final amount of chlorine dioxide produced increases with increase in acid concentrations. [PTU $]_{0}=2.00 \times 10^{-4} \mathrm{M}$, $\left[\mathrm{ClO}_{2}{ }^{-}\right]_{0}=2.00 \times 10^{-3} \mathrm{M},\left[\mathrm{H}^{+}\right]_{0}=$ (a) $0.0025 \mathrm{M}$, (b) $0.005 \mathrm{M}$, (c) $0.0075 \mathrm{M}$, (d) $0.01 \mathrm{M}$ and (e) $0.015 \mathrm{M}$ 
Figure 5.3b shows a series of experiments undertaken at low acid concentrations showing, a general increase in the amount of chlorine dioxide produced as initial acid concentrations are increased. However, between acid concentrations of $0.015 \mathrm{M}$ and $0.020 \mathrm{M}$, this trend is reversed (figure 5.3c) and increasing acid concentrations further resulted in production of reduced amounts of chlorine dioxide. Separation of figures $5.3 \mathrm{~b}$ and $5.3 \mathrm{c}$ is to enable us to best display this reversal in trend.

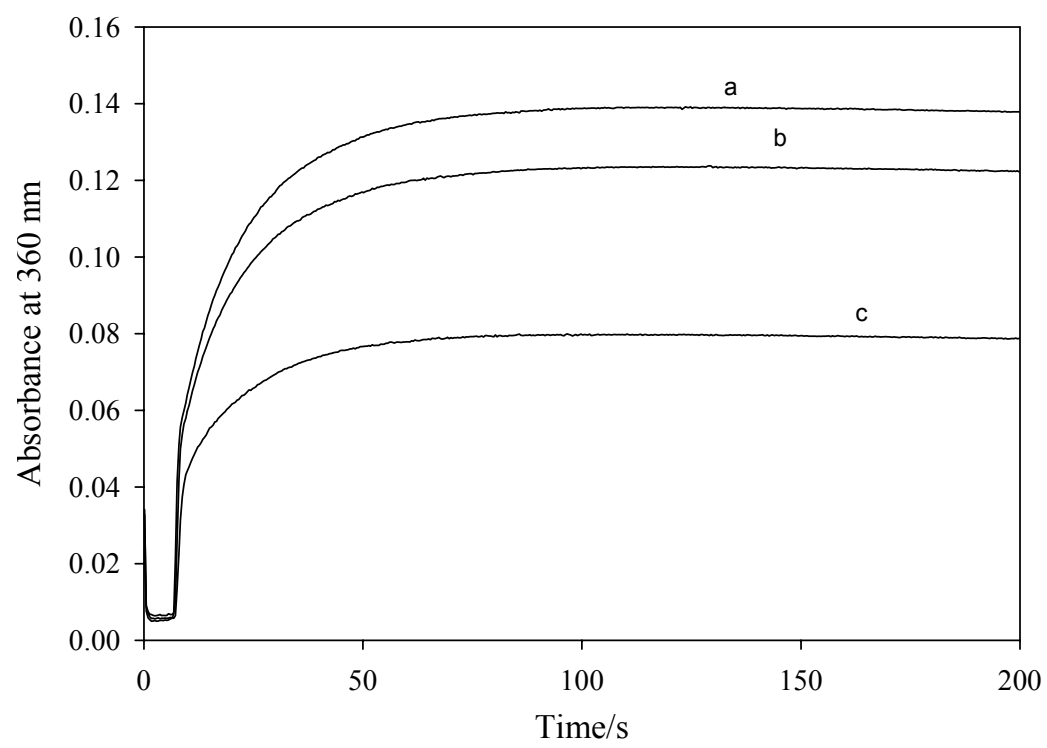

Figure 5.3c: Effect of acid at high oxidant to reductant ratios; $\left[\mathrm{ClO}_{2}{ }^{-}\right]_{0} /[\mathrm{PTU}]_{0}=10.0$. In this case there is a well induction period which is still not affected by the amount of acid. Concentration of acid, however, is now inversely proportional to the amount of chlorine dioxide formed. $[\mathrm{PTU}]_{0}=2.00 \times 10^{-4} \mathrm{M},\left[\mathrm{ClO}_{2}^{-}\right]_{0}=2.00 \times 10^{-3} \mathrm{M},\left[\mathrm{H}^{+}\right]_{0}=$ (a) $0.02 \mathrm{M}$, (b) $0.02 \mathrm{M}$ and (c) $0.05 \mathrm{M}$.

\subsubsection{The chlorite-phenylthiourea ratio}

There were two ways of altering oxidant to reductant ratio, R. One involves fixing the initial chlorite concentrations and varying $[\mathrm{PTU}]_{0}$ (see figure 5.4), and the other involves altering both $\left[\mathrm{ClO}_{2}^{-}\right]_{0}$ and $[\mathrm{PTU}]_{0}$ simultaneously (see figure 5.5). In Figure 5.4, traces $\mathrm{c}, \mathrm{d}$ and $\mathrm{e}$ had $\mathrm{R}<2$; and so, ultimately, at $\mathrm{t}_{\infty}$, the final chlorine dioxide 
concentrations decayed to zero. The observed chlorine dioxide formation within the first $30 \mathrm{~s}$ of this reaction was only transient.

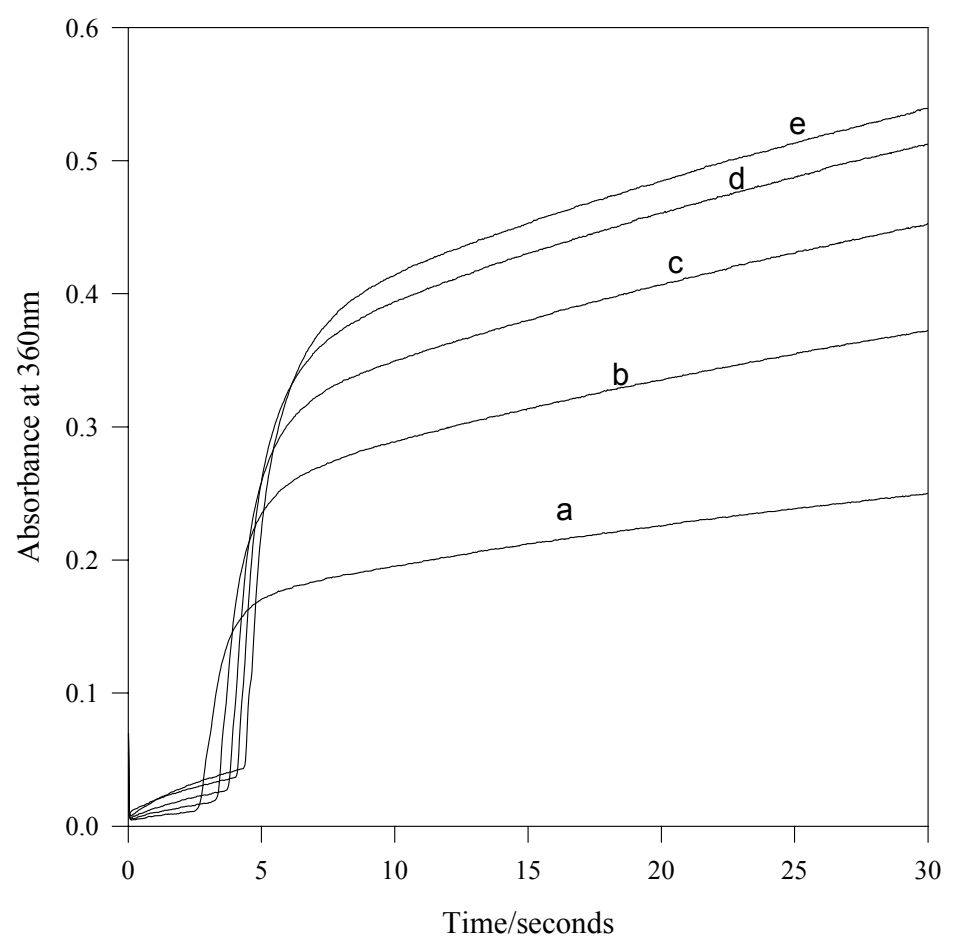

Figure 5.4: Effect of varying $[\mathrm{PTU}]_{0}$ on the formation of chlorine dioxide at fixed chlorite concentrations. Concentration of PTU is directly proportional to amount of chlorine dioxide produced and the induction period at fixed chlorite concentrations. $\left.\left[\mathrm{ClO}_{2}^{-}\right]_{0}=5.00 \times 10^{-3} \mathrm{M}, \mathrm{H}^{+}\right]_{0}$ $=0.125 \mathrm{M},[\mathrm{PTU}]_{0}=$ (a) $2.5 \times 10^{-4} \mathrm{M}$, (b) $5.0 \times 10^{-4} \mathrm{M}$, (c) $7.5 \times 10^{-4} \mathrm{M}$, (d) $10.0 \times 10^{-4} \mathrm{M}$ and (e) $12.5 \times 10^{-4} \mathrm{M}$

As expected, higher PTU concentrations gave longer induction periods and faster rates of formation of chlorine dioxide. This is to be expected if the reactive species that control the production of chlorine dioxide are derived from the oxidation of PTU or reduction of $\mathrm{ClO}_{2}^{-}$. Figure 5.5 shows a wider range of ratios; $10>\mathrm{R}>1.7$. Lower ratios, e.g. traces a, $\mathrm{b}$ and $\mathrm{c}$ in figure 5.5 gave a transient formation of chlorine dioxide, while higher ratios gave a monotonic increase in chlorine dioxide. Figure 5.5 can be regarded as an extension of the time scale shown in figure 5.4. 


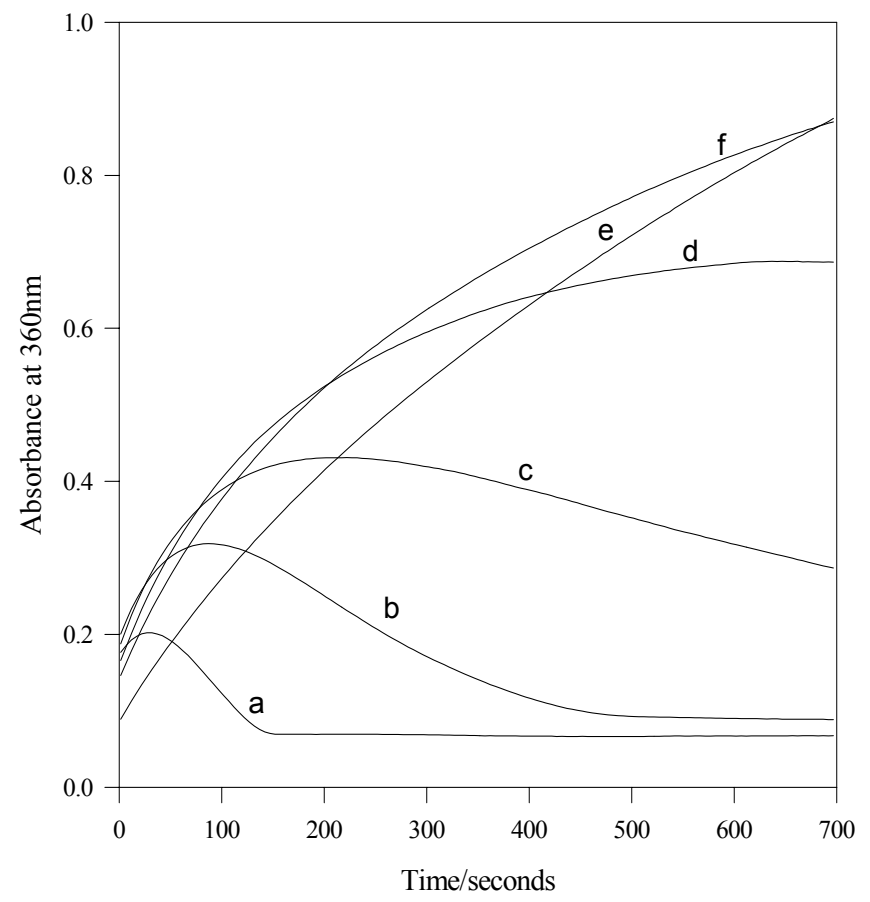

Figure 5.5: The effect of $\left[\mathrm{ClO}_{2}{ }^{-}\right]_{0} /[\mathrm{PTU}]_{0}$ ratio on the absorbance traces at $360 \mathrm{~nm}$. At high ratios of 4 and above, there is a monotonic formation of chlorine dioxide. At lower ratios, there is transient formation of chlorine dioxide. $\left[\mathrm{HClO}_{4}\right]_{0}=0.125 \mathrm{M},\left[\mathrm{ClO}_{2}^{-}\right]_{0}=2.5 \times 10^{-3} \mathrm{M}$. $[\mathrm{PTU}]_{0}=$ (a) $1.5 \times 10^{-3} \mathrm{M}$, (b) $1.25 \times 10^{-3} \mathrm{M}$, (c) $1.0 \times 10^{-3} \mathrm{M}$, (d) $7.5 \times 10^{-4} \mathrm{M}$, (e) $5.0 \times 10^{-4} \mathrm{M}$ and (f) $2.5 \times 10^{-4} \mathrm{M}$

\subsubsection{Chlorine dioxide formation}

Previous studies that involve chlorite oxidations have used the formation of chlorine dioxide as a means of following the reaction and evaluating kinetic constants [20]. Chlorine dioxide is formed in oxychlorine-organosulfur reaction systems, by an extraneous and purely oxyhalogen reaction that does not directly involve the substrate that is being oxidized [25,26]. In non-radical processes, the first reactive oxyhalogen species formed in chlorite oxidations is hypochlorous acid, $\mathrm{HOCl}$ [23]. $\mathrm{HOCl}$ will then react very rapidly with remaining chlorite to produce chlorine dioxide [24]: 


$$
2 \mathrm{ClO}_{2}^{-}+\mathrm{HOCl}+\mathrm{H}^{+} \rightarrow 2 \mathrm{ClO}_{2}(\text { aq, yellow })+\mathrm{Cl}^{-}+\mathrm{H}_{2} \mathrm{O}
$$

Three factors will determine whether chlorine dioxide is formed: (1) if the rate of reaction between the reducing substrate and chlorine dioxide is fast, then there will be no observation of chlorine dioxide until all the reducing substrate and its oxidation intermediates have been completely oxidized; (2) in high ratios, $\mathrm{R}>4$ (which is much higher than the required stoichiometry of $\mathrm{R}=2$ ), production of $\mathrm{ClO}_{2}$ will overwhelm the small concentrations of substrate present such that there will be an immediate formation of $\mathrm{ClO}_{2}$. Transient and complex $\mathrm{ClO}_{2}$ formation can be obtained at low ratios, typically below 4; and (3) if rate of reaction of chlorine dioxide with the reducing substrate is slow, then oligooscillatory behavior will be obtained for reaction solutions with $\mathrm{R}<2$. A clear illustration of this can be seen in the data shown in Figure 5.5. Monotonic $\mathrm{ClO}_{2}$ formation is obtained at ratios above 2.5 for acid concentrations of $0.125 \mathrm{M}$. This threshold ratio will change depending on the $\mathrm{pH}$ conditions. After long incubation times, all experiments with ratios below 2 will show no final chlorine dioxide accumulations (see stoichiometry 5.1 and figure 5.5). The observed transient chlorine dioxide formation at these ratios clearly shows the fragile balance between reactions that form chlorine dioxide, and those that consume it. The effect of chlorite concentrations (effectively, this is a variation in the ratio, $\mathrm{R}$ ) on the formation of chlorine dioxide is shown in the data in figures 5.2a and 5.5. Traces with $\mathrm{R} \leq 2$ will eventually have no chlorine dioxide at $\mathrm{t}_{\infty}$. For a fixed amount of acid concentrations, the rate of formation and transient amount of chlorine dioxide formed is directly proportional to initial chlorite concentrations. This is to be expected if the formation of chlorine dioxide is mainly through reaction 5.5. The 
effect of acid on the reaction will be complex due to the fact that acid influences nearly all elementary reactions in this reaction mixture. In some reactions it is catalytic, and in some, inhibitory. Three main features of chlorine dioxide formation all change with acid: rate of formation of chlorine dioxide, maximum transient chlorine dioxide formed, and the rate of consumption of chlorine dioxide after attaining its peak. Ultimately, the overall global reaction dynamics will not show any specific dependence on acid. Figure 5.3a shows the high acid environment in which acid is inhibitory in the formation of chlorine dioxide. The ratio used in these data, $\mathrm{R}=4$, does not give an overwhelming excess of the oxidant, and thus the rate of consumption of $\mathrm{ClO}_{2}$ by PTU and other reductants has to be factored into the determination of the overall reaction dynamics. Figure 5.3b, however, with $\mathrm{R}=10$, makes the observed chlorine dioxide formation a pure oxyhalogen reaction since most of the reducing organosulfur compound and its intermediates will have been completely consumed by the time chlorine dioxide is formed.

\subsubsection{Oxidations by chlorine dioxide}

Figures 5.6a, 5.6b and 5.6c show the reaction of chlorine dioxide with PTU monitored at $360 \mathrm{~nm}$. The reaction displays what appears to be a biphasic behavior, with, initially, sigmoidal autocatalytic kinetics, followed by normal decay (follow trace $\mathrm{c}$ in figure 5.6a and all traces in figures 5.6b and 5.6c). In excess of PTU, only the initial (autocatalytic) phase is observed (figure 5.6a, traces a and b). Such sigmoidal decay kinetics have been consistently observed with respect to chlorine dioxide oxidations [27]. Figure 5.6b shows absorbance traces obtained in excess $\mathrm{ClO}_{2}$. The autocatalytic phase 
lasts until a stoichiometric ratio of $4: 5$ is attained; i.e. 4 moles of $\mathrm{ClO}_{2}$ to 5 moles of PTU. These data are summarized in Table 5.1. This ratio suggests that autocatalysis lasts until all the PTU has been oxidized to the sulfinic acid (see reaction stoichiometry 5.28), after which, a slow monotonic decay of chlorine dioxide is then observed right up to the end of the reaction.

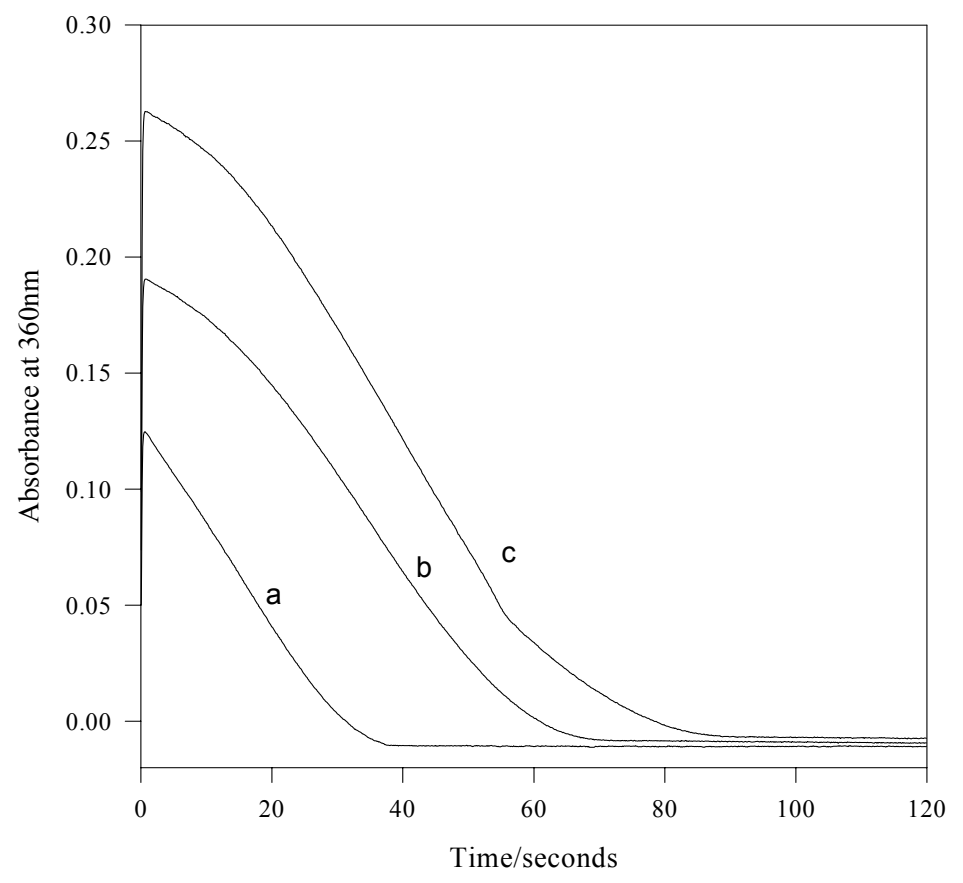

Figure 5.6a: Direct reaction of PTU and chlorine dioxide monitored at $360 \mathrm{~nm}$ with oxidant to reductant ratios below stochiometric amount. A single sigmoidal decay for the consumption of chlorine dioxide is observed with all chlorine dioxide being depleted at the end of the reaction. $[\mathrm{PTU}]_{0}=2.5 \times 10^{-4} \mathrm{M},\left[\mathrm{H}^{+}\right]_{0}=0.125 \mathrm{M},\left[\mathrm{ClO}_{2}\right]_{0}=$ (a) $2.0 \times 10^{-4} \mathrm{M}$, (b) $3.0 \times 10^{-4} \mathrm{M}$ and (c) $4.0 \times 10^{-4}$ $\mathrm{M}$.

A simple experiment was undertaken in which the synthesized sulfinic acid of PTU, phenylthiourea dioxide, (phenylaminoiminomethanesulfinic acid) was reacted directly with chlorine dioxide. This experiment showed that the reaction of the sulfinic acid and chlorine dioxide was exceedingly slow, much slower than the reaction of chlorine dioxide with PTU. 
Previous reports on chlorine dioxide oxidations have suggested that the initial part of the reaction involves the formation of $\mathrm{ClO}_{2}^{-}$with subsequent oxidations being undertaken by chlorite [28]. Addition of chlorite in $\mathrm{ClO}_{2}$ oxidations should thus show a catalytic effect.

Table 5.1: Analysis of absorbance data from Figure 6b. This table shows that the reaction slows down after a nearly quantitative formation of the sulfinic acid, indicating that the reaction between $\mathrm{ClO}_{2 \text { (aq) }}$ and the sulfinic acid is very slow. This is also the position when chlorine dioxide formation commences (see figure 5.10).

\begin{tabular}{llllll}
\hline$\left[\mathrm{ClO}_{2}\right]_{0}$ & {$[\mathrm{PTU}]_{0}$} & $\begin{array}{l}{\left[\mathrm{ClO}_{2}\right]_{\mathrm{t}} \text { at }} \\
\text { end of } \\
\text { autocatalysis }\end{array}$ & $\begin{array}{l}{\left[\mathrm{ClO}_{2}\right] /} \\
{[\mathrm{PTU}]}\end{array}$ & $\begin{array}{l}\text { Expected } \\
{\left[\mathrm{ClO}_{2}\right] /[\mathrm{PTU}]} \\
\text { for formation } \\
\text { of sulfinic } \\
\text { acid }\end{array}$ & $\begin{array}{l}\text { Deviation } \\
\text { observed }\end{array}$ \\
\hline $0.000600 \mathrm{M}$ & $0.00025 \mathrm{M}$ & $0.000418 \mathrm{M}$ & $0.728 \mathrm{M}$ & 0.800 & $7.20 \%$ \\
$0.000574 \mathrm{M}$ & $0.00025 \mathrm{M}$ & $0.000387 \mathrm{M}$ & $0.748 \mathrm{M}$ & 0.800 & $5.20 \%$ \\
& $0.00025 \mathrm{M}$ & $0.000328 \mathrm{M}$ & $0.744 \mathrm{M}$ & 0.800 & $5.60 \%$ \\
$0.000514 \mathrm{M}$ & $0.00025 \mathrm{M}$ & $0.000278 \mathrm{M}$ & $0.748 \mathrm{M}$ & 0.800 & $5.20 \%$ \\
\hline
\end{tabular}




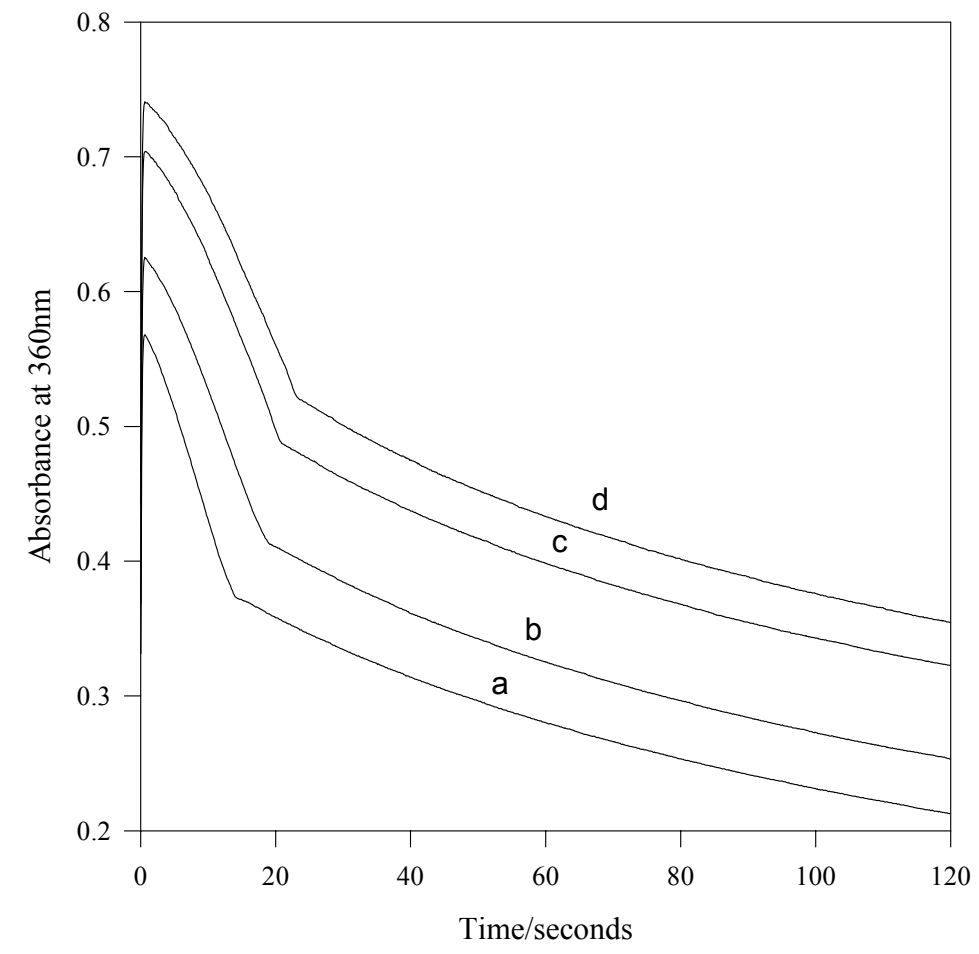

Figure 5.6b: Chlorine dioxide oxidation at oxidant to reductant ratios; $\left[\mathrm{ClO}_{2}^{-}\right]_{0} /[\mathrm{PTU}]_{0}>2$. This shows two distinct steps of an initial sigmoidal decay followed by a standard decay. [PTU $]_{0}=2.5$ x $10^{-4} \mathrm{M},\left[\mathrm{H}^{+}\right]_{0}=0.125 \mathrm{M},\left[\mathrm{ClO}_{2}\right]_{0}=(\mathrm{a}) 7 \times 10^{-4} \mathrm{M}$, (b) $=8 \times 10^{-4} \mathrm{M}$, (c) $9 \times 10^{-4} \mathrm{M}$ and (e) $=10 \mathrm{x}$ $10^{-4} \mathrm{M}$

Data in figure 5.6c indicates that this, indeed, is the case. Only micromolar quantities of chlorite were used in this series of experiments, and yet the effect is quite noticeable. The initial autocatalytic part of the reaction is the one that is immediately catalyzed with the rest of the reaction not catalyzed any further. This is evidence that chlorite is indeed the dominant pathway utilized by chlorine dioxide oxidations. The parallel nature of the absorbance traces after addition of chlorite also suggests that chlorite is not an autocatalytic species since its catalytic effect is linearly dependent on the amount of chlorite anions added. 


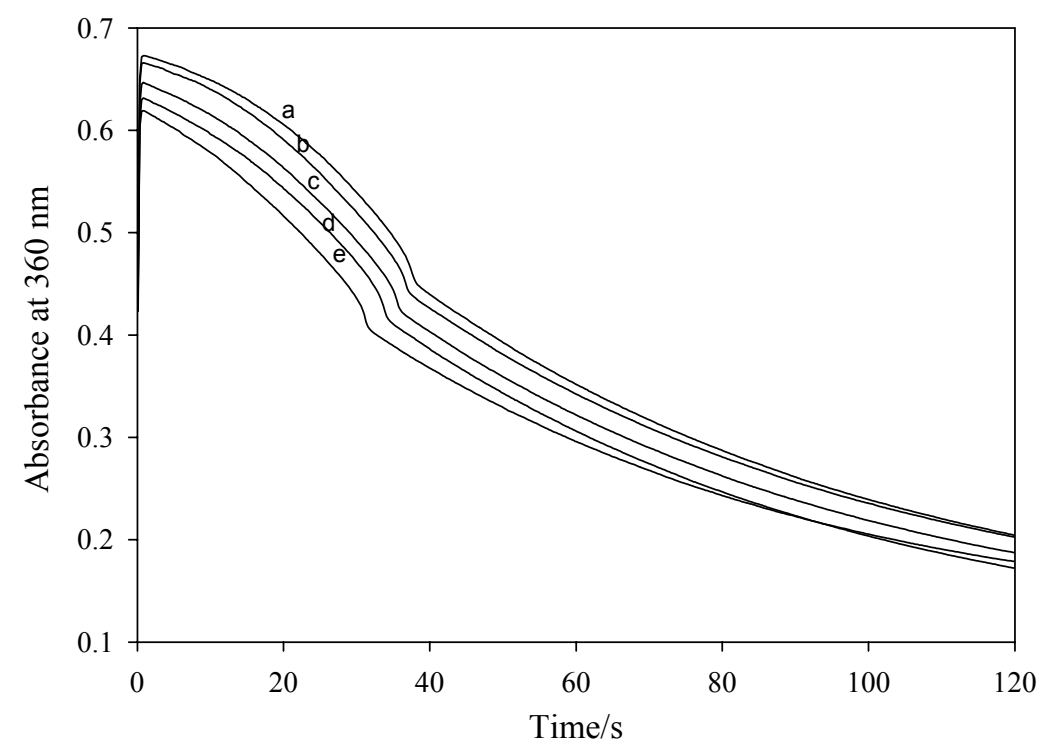

Figure 5.6c: Effect of chlorite on the oxidation of PTU by chlorine dioxide. The data also displays two-stage kinetics. Chlorite lowers the rate of the first step, (sigmoidal decay) as shown by traces (b) - (e). $[\mathrm{PTU}]_{0}=2.5 \times 10^{-4} \mathrm{M},\left[\mathrm{ClO}_{2}\right]_{0}=6.76 \times 10^{-4} \mathrm{M},\left[\mathrm{H}^{+}\right]_{0}=0.125 \mathrm{M},\left[\mathrm{ClO}_{2}^{-}\right]_{0}=$ (a) 0 , (b) $5 \times 10^{-6} \mathrm{M}$ (c) $1.5 \times 10^{-5} \mathrm{M}$, (d) $3.0 \times 10^{-5} \mathrm{M}$ and (e) $5.0 \times 10^{-5} \mathrm{M}$

\subsubsection{Data collected at $249 \mathrm{~nm}$}

Figure 5.1 shows that PTU has a sharp and isolated peak in its UV spectrum at $249 \mathrm{~nm}$. The products of its oxidation, phenylurea, as well as chlorine dioxide, also absorb at this wavelength, but with lower extinction coefficients. Data collected at this wavelength can deliver unambiguous initial rates of consumption of PTU at the beginning of the reaction before the reaction product starts to accumulate in the reaction medium. Figure 5.7a shows a typical series of experiments performed at $249 \mathrm{~nm}$. They show an initial rapid decrease in absorbance signal which quickly damps out to give way to a slower decrease in absorbance. This sequence would appear to be the opposite of that observed in figures 5.6a, 5.6b and 5.6c. The damping out in the decrease in absorbance is due to the formation of an intermediate which is long-lived enough to be able to accumulate. If one compares comparable data from figures $5.7 \mathrm{a}$ and $5.6 \mathrm{~b}$, one 
notices that the onset of damping in Figure 5.7a coincides with the end of the autocatalytic phase in figure 5.6b. The very sharp and abrupt halt in decrease in absorbance shown in figure $5.7 \mathrm{a}$ is due to the formation of the product which contributes substantially to the final absorbance observed.

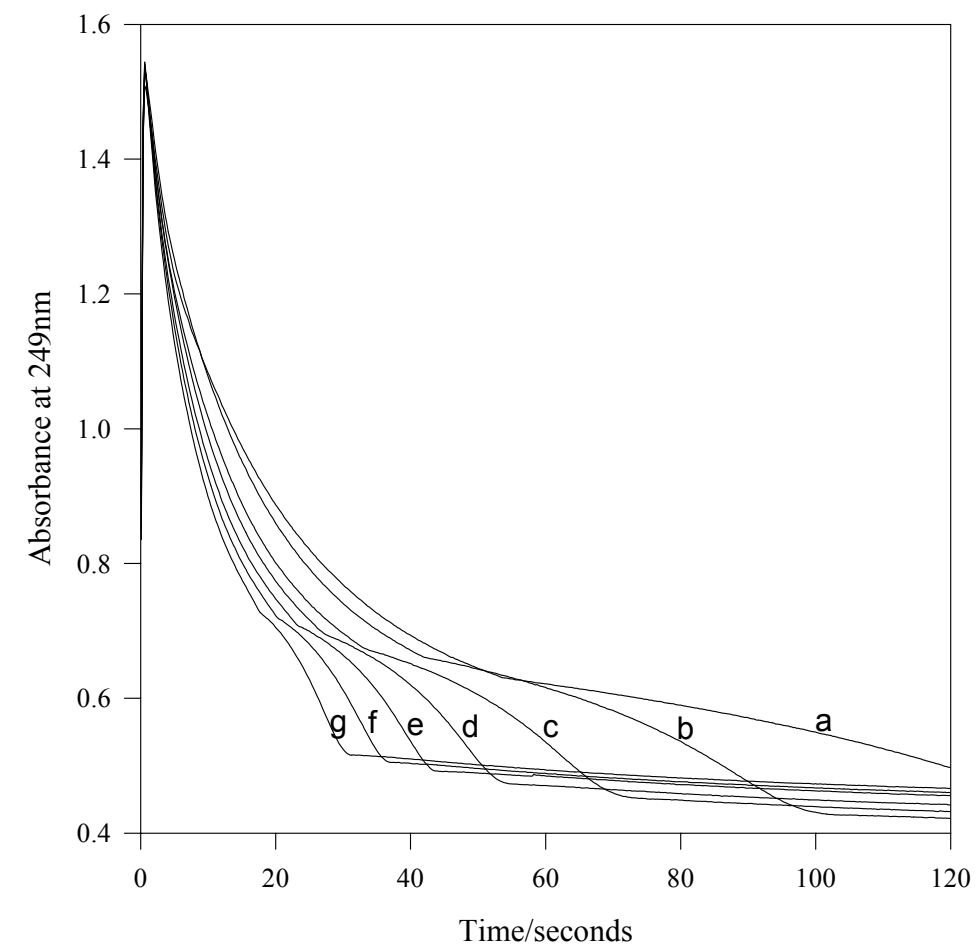

Figure 5.7a: Depletion of PTU monitored at $249 \mathrm{~nm}$ for the oxidation by chlorite. These data also display two-stage kinetics. The product of PTU oxidation gives the residual absorbance that can be seen in the absorbance-time data. $\quad[\mathrm{PTU}]_{0}=1.25 \times 10^{-4} \mathrm{M},\left[\mathrm{HClO}_{4}\right]_{0}=0.125 \mathrm{M},\left[\mathrm{ClO}_{2}{ }^{-}\right]=$ (a) $4.0 \times 10^{-4} \mathrm{M}$, (b) $5.0 \times 10^{-4} \mathrm{M}$, (c) $6.0 \times 10^{-4} \mathrm{M}$, (d) $7.0 \times 10^{-4} \mathrm{M}$, (e) $8.0 \times 10^{-4} \mathrm{M}$, (f) $9.0 \times 10^{-4} \mathrm{M}$ and $(\mathrm{g}) 10.0 \times 10^{-4} \mathrm{M}$

The data in figure 5.7a gives a linear dependence of the initial rate of PTU consumption with initial chlorite concentrations (figure 5.7b). Induction period data (see figure 5.2b) had also suggested a first order dependence of the reaction rate with chlorite concentrations. Direct oxidation of PTU by chlorine dioxide, monitored at $249 \mathrm{~nm}$, gives traces that display autocatalysis (figure 5.7c). This is in agreement with the data 
obtained in figures 5.6a-5.6c. Figure 5.8a shows the same types of absorbance traces as those shown in figure $5.7 \mathrm{a}$ but with variable acid concentrations. Contrary to the data obtained at $360 \mathrm{~nm}$ with chlorine dioxide, the depletion kinetics of PTU are monotonic and less complex.

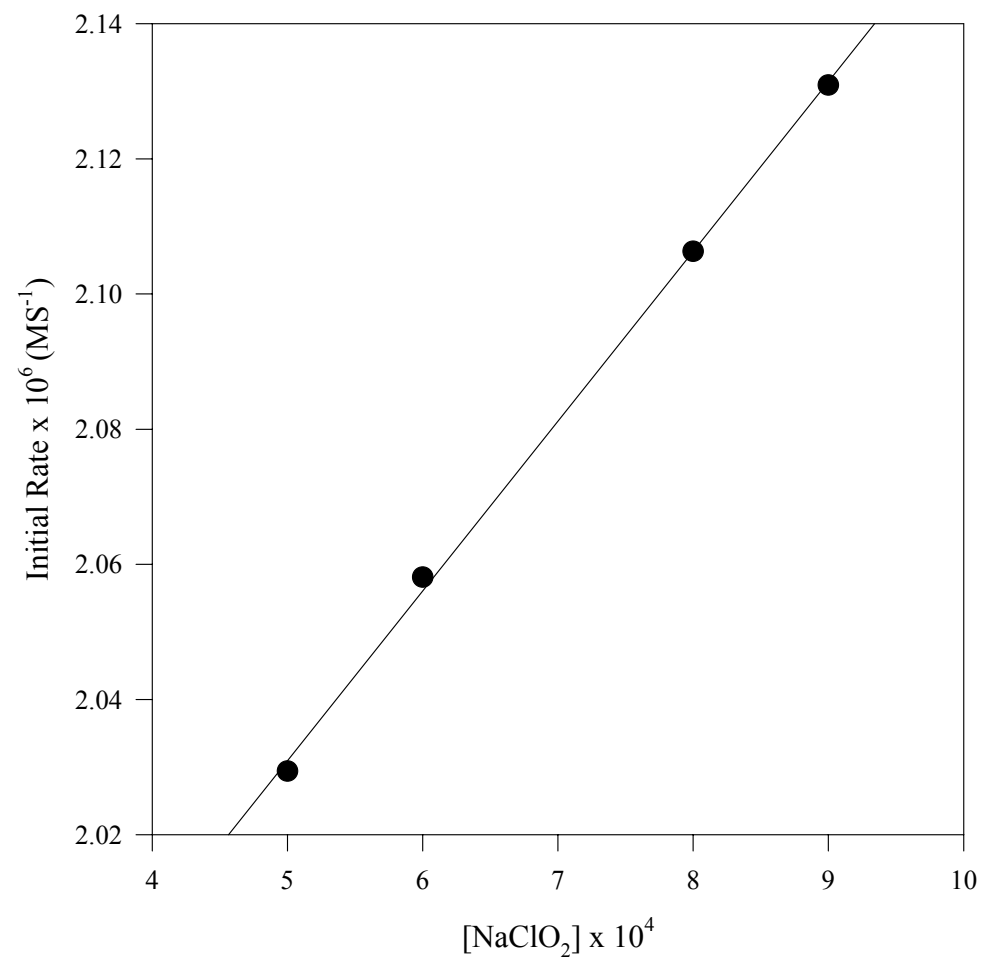

Figure 5.7b: Relationship between initial rate of consumption of PTU and the initial chlorite concentrations. There is a solid linear dependence. $[\mathrm{PTU}]_{0}=1.25 \times 10^{-4} \mathrm{M},\left[\mathrm{HClO}_{4}\right]_{0}=0.125 \mathrm{M}$

Figures 5.8a and 5.8b show the effects of acid on the initial rate of consumption of PTU. Low acid concentrations display a saturation at about $0.02 \mathrm{M} \mathrm{HClO}_{4}$ (figure 5.8b). If the acid is further increased, a retardation is observed. Figure 5.8c summarizes the acid effect by combining a series of acid concentrations ranging from $0.025 \mathrm{M}$ to $0.50 \mathrm{M}$. Very low acid concentrations show a sigmoidal autocatalytic decay of the peak at $249 \mathrm{~nm}$ (not shown in figure 5.8c), and a gradual increase in acid will change the kinetics traces 
from this convex shape to a straight line zero order type decay kinetics (trace a) and finally to the standard concave decay kinetics curves which are observed at very high acid concentrations (traces $\mathrm{d}-\mathrm{f}$ ).

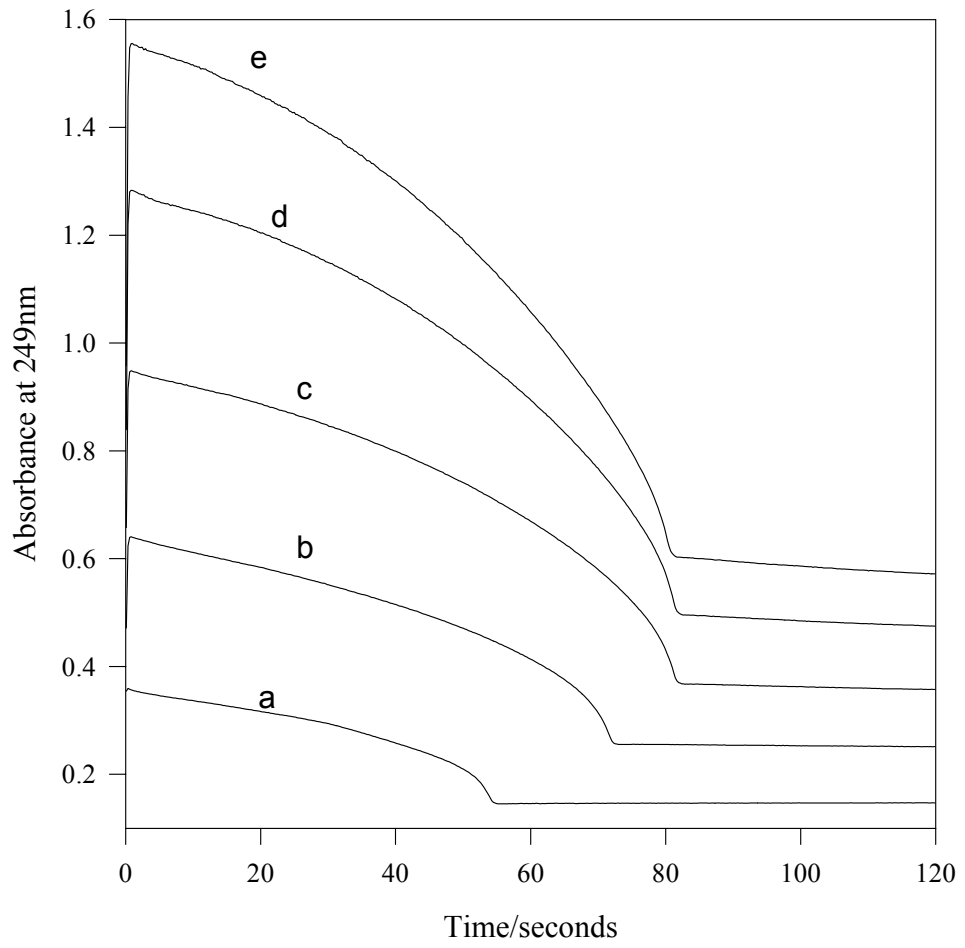

Figure 5.7c: Depletion of PTU in its oxidation by chlorine dioxide monitored at $249 \mathrm{~nm}$. In high excess chlorine dioxide environments (ratios $>3$ ), a single sigmoidal decay is observed for the consumption of PTU. $\left[\mathrm{ClO}_{2}\right]_{0}=4.27 \times 10^{-4} \mathrm{M},\left[\mathrm{H}^{+}\right]_{0}=0.125 \mathrm{M},[\mathrm{PTU}]_{0}=$ (a) $2.5 \times 10^{-5} \mathrm{M}$, (b) $5.0 \mathrm{x}$ $10^{-5} \mathrm{M}$ (c) $7.5 \times 10^{-5} \mathrm{M}$, (d) $10 \times 10^{-5} \mathrm{M}$ and (e) $12.5 \times 10^{-5} \mathrm{M}$.

The important aspect of figure $5.8 \mathrm{c}$ to note is that the second phase of the reaction is much faster in low acid concentrations, while in high acid concentrations this intermediate species formed lingers much longer before forming products. 


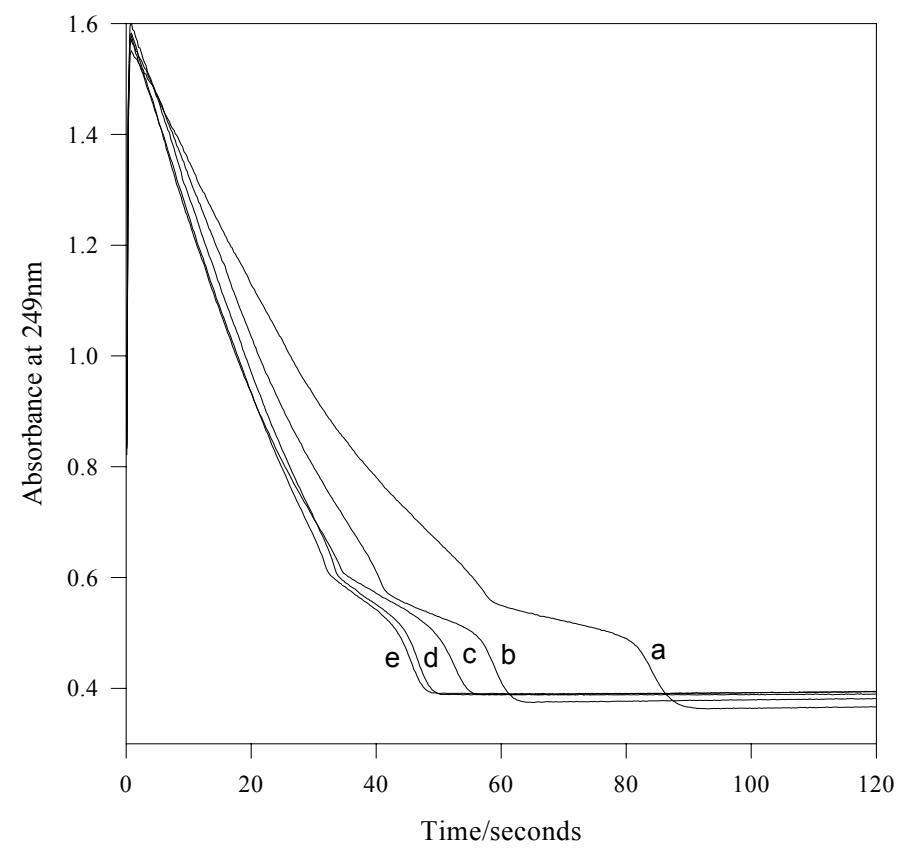

Figure 5.8a: Absorbance traces at $249 \mathrm{~nm}$ showing the effect of acid on the rate of consumption of PTU at low acid concentrations. Acid catalyzes the first stage of the reaction but appears to retard the second stage. $[\mathrm{PTU}]_{0}=1.25 \times 10^{-4} \mathrm{M},\left[\mathrm{ClO}_{2}^{-}\right]_{0}=5.0 \times 10^{-4} \mathrm{M}\left[\mathrm{HClO}_{4}\right]_{0}=$ (a) $0.005 \mathrm{M}$, (b) $0.010 \mathrm{M}$, (c) $0.015 \mathrm{M}$, (d) $0.020 \mathrm{M}$, and (e) $0.025 \mathrm{M}$.

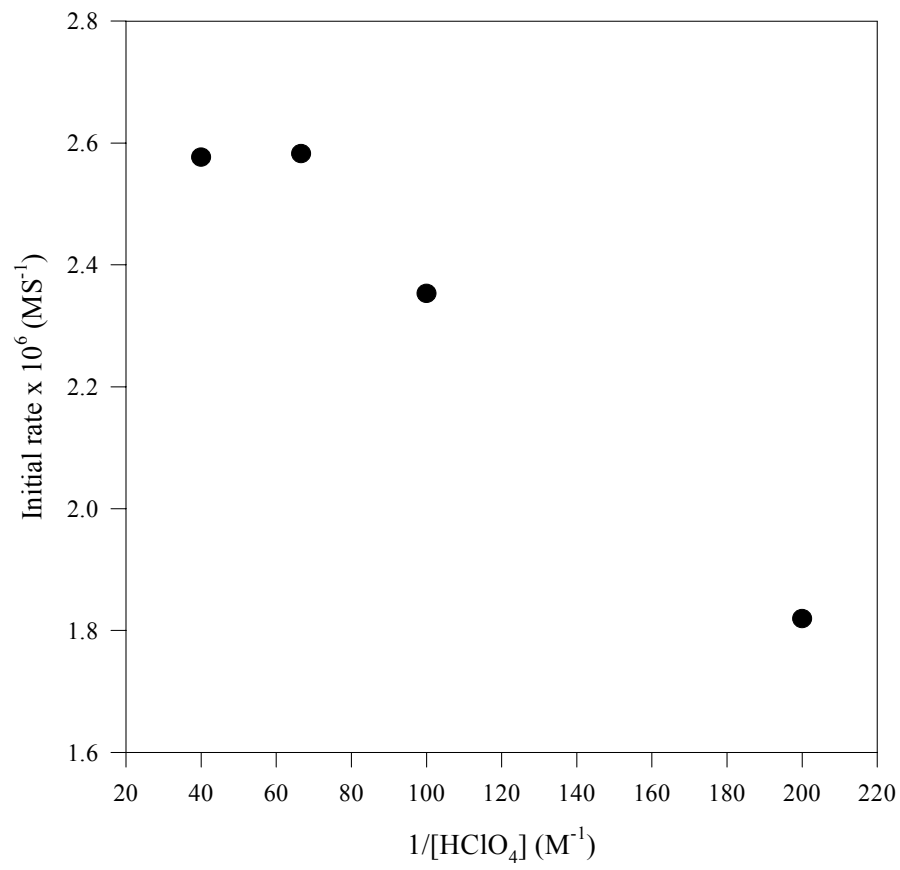

Figure 5.8b: Initial rate plots for low acid concentrations. A maximum rate of consumption is attained at approximately $\mathrm{pH} 2.00$. Higher acid concentrations show a retardation of the rate of consumption of PTU. 


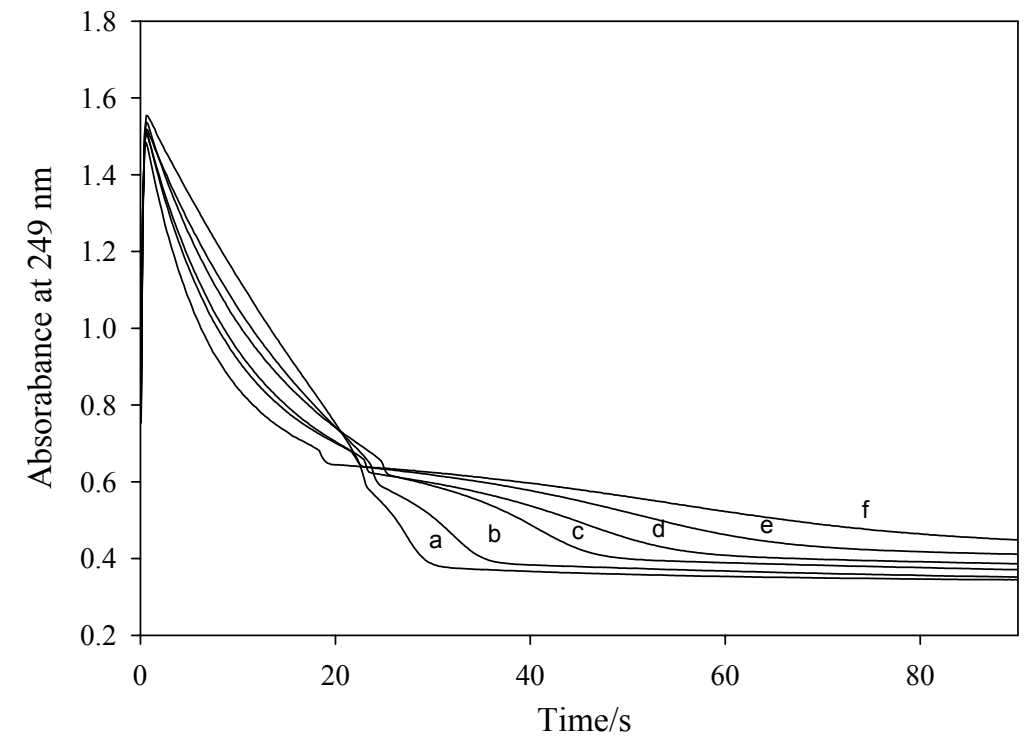

Figure 5.8c: Absorbance traces at $249 \mathrm{~nm}$ showing the effect of acid on the rate of consumption of PTU at high acid concentrations. Acid still catalyzes the first stage of the reaction but appears to retard the second stage. $[\mathrm{PTU}]_{0}=1.25 \times 10^{-4} \mathrm{M},\left[\mathrm{ClO}_{2}^{-}\right]_{0}=7.0 \times 10^{-4} \mathrm{M}\left[\mathrm{HClO}_{4}\right]_{0}=$ (a) $0.025 \mathrm{M}$, (b) $0.050 \mathrm{M}$, (c) $0.10 \mathrm{M}$, (d) $0.15 \mathrm{M}$, (e) $0.20 \mathrm{M}$ and (f) $0.50 \mathrm{M}$.

\subsubsection{Chlorine dioxide oxidations monitored at $249 \mathrm{~nm}$}

Figures 5.9a $-5.9 \mathrm{c}$ show the depletion kinetics of PTU with chlorine dioxide as the oxidant. Figure 5.9a shows the dominance of sigmoidal kinetics even in excess oxidant conditions, although the observed autocatalysis decreased with increasing oxidant concentrations. Highly excess of oxidant, however, still did not afford pseudo-first order kinetics. In highly excess the oxidant concentrations, the depletion kinetics of PTU approached zero order. 


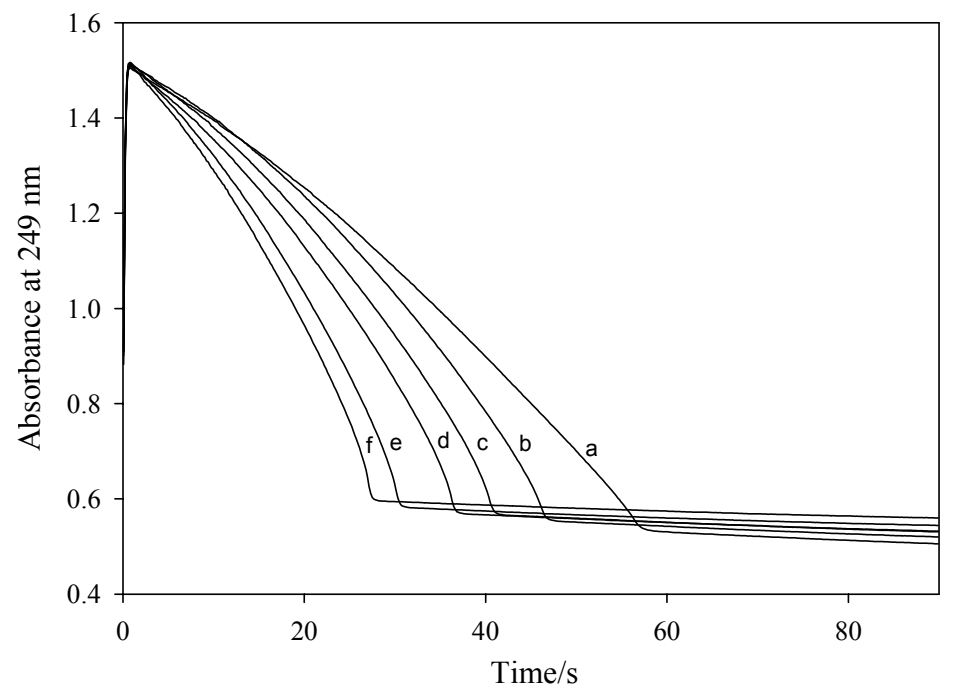

Figure 5.9a: Chlorine dioxide oxidation: A single sigmoidal decay curve for the consumption of PTU at $249 \mathrm{~nm}$ is observed in excess chlorine dioxide environments. Rate of PTU depletion increases with increase in chlorine dioxide concentrations. $[\mathrm{PTU}]_{0}=1.25 \times 10^{-4} \mathrm{M},\left[\mathrm{HClO}_{4}\right]_{0}=$ $0.125 \mathrm{M}$. $\left[\mathrm{ClO}_{2}\right]_{0}=$ (a) $1.69 \times 10^{-4} \mathrm{M}$, (b) $3.38 \times 10^{-4} \mathrm{M}$, (c) $4.225 \times 10^{-4} \mathrm{M}$, (d) $5.07 \times 10^{-4} \mathrm{M}$, (e) 6.76 x $10^{-4} \mathrm{M}$ and (f) $8.45 \times 10^{-4} \mathrm{M}$.

Figure $5.9 \mathrm{~b}$ shows the effect of low acid concentrations. Acid, at these conditions, $1.3<$ $\mathrm{pH}<2.7$, is very effective in inhibiting the reaction with respect to rate of consumption of PTU. Figure 5.9c shows that if acid is increased further, its inhibition effect saturates and it begins to catalyze the reaction. High acid concentrations, however, could not catalyze the reaction such that is became as fast as it is in low acid environments. 


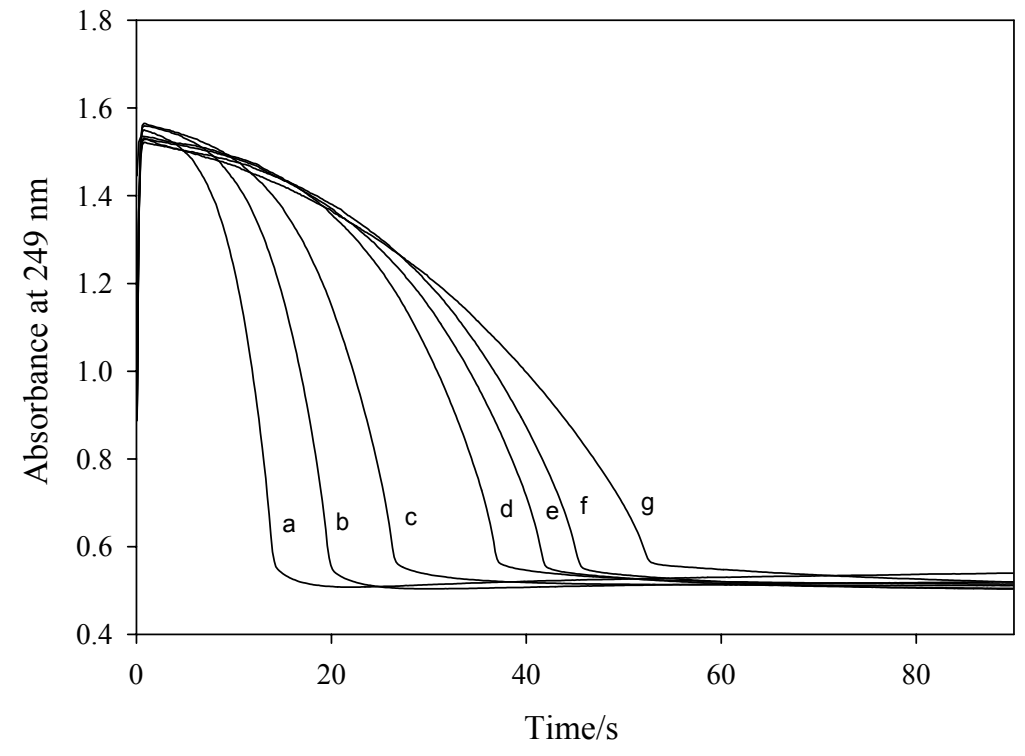

Figure 5.9b: Chlorine dioxide oxidation: At low acid concentrations, acid inhibits the oxidation of PTU by chlorine dioxide at $249 \mathrm{~nm}$. Sigmoidal autocatalytic decay kinetics are much more pronounced at low acid concentrations. $[\mathrm{PTU}]_{0}=1.25 \times 10^{-4} \mathrm{M},\left[\mathrm{ClO}_{2}\right]_{0}=3.38 \times 10^{-4} \mathrm{M} \cdot\left[\mathrm{HClO}_{4}\right]_{0}$ = (a) $0.0025 \mathrm{M}$, (b) $0.005 \mathrm{M}$, (c) $0.010 \mathrm{M}$, (d) $0.015 \mathrm{M}$, (e) $0.020 \mathrm{M}$, (f) $0.025 \mathrm{M}$ and (g) 0.050 M.

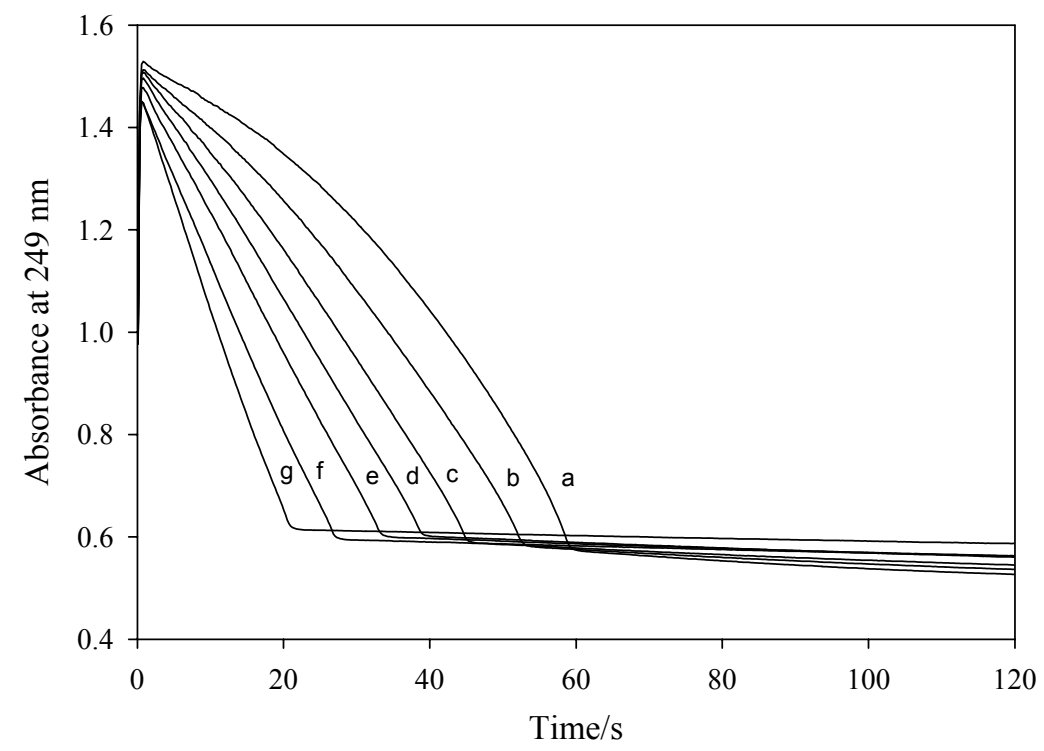

Figure 5.9c: Chlorine dioxide oxidation: At high acid concentrations, acid catalyzes the oxidation of PTU by chlorine dioxide at $249 \mathrm{~nm}$. Sigmoidal autocatalytic decay kinetics are still more pronounced at low acid concentrations. $[\mathrm{PTU}]_{0}=1.25 \times 10^{-4} \mathrm{M},\left[\mathrm{ClO}_{2}\right]_{0}=3.38 \times 10^{-4} \mathrm{M}$. $\left[\mathrm{HClO}_{4}\right]_{0}$ $=$ (a) $0.10 \mathrm{M}$, (b) $0.15 \mathrm{M}$, (c) $0.20 \mathrm{M}$, (d) $0.25 \mathrm{M}$, (e) $0.30 \mathrm{M}$, (f) $0.40 \mathrm{M}$ and (g) $0.50 \mathrm{M}$. 


\subsubsection{Combining data at $360 \mathrm{~nm}$ and $249 \mathrm{~nm}$}

Figure 5.10 superimposes data collected at $249 \mathrm{~nm}$ and at $360 \mathrm{~nm}$. This shows that chlorine dioxide formation commences at the start of the second phase of the reaction when monitored at $249 \mathrm{~nm}$. This is an important experimental observation: whatever intermediate is formed or accumulated during the second phase of the reaction; it must be inert to (or reacts very slowly with) chlorine dioxide (hence its accumulation). This fact, combined with the experiment that showed a very slow reaction between chlorine dioxide and phenylthiourea sulfinic acid as well as data shown in figure 5.6b (summarized in Table 5.1) indicates that the intermediate species formed is the sulfinic acid.

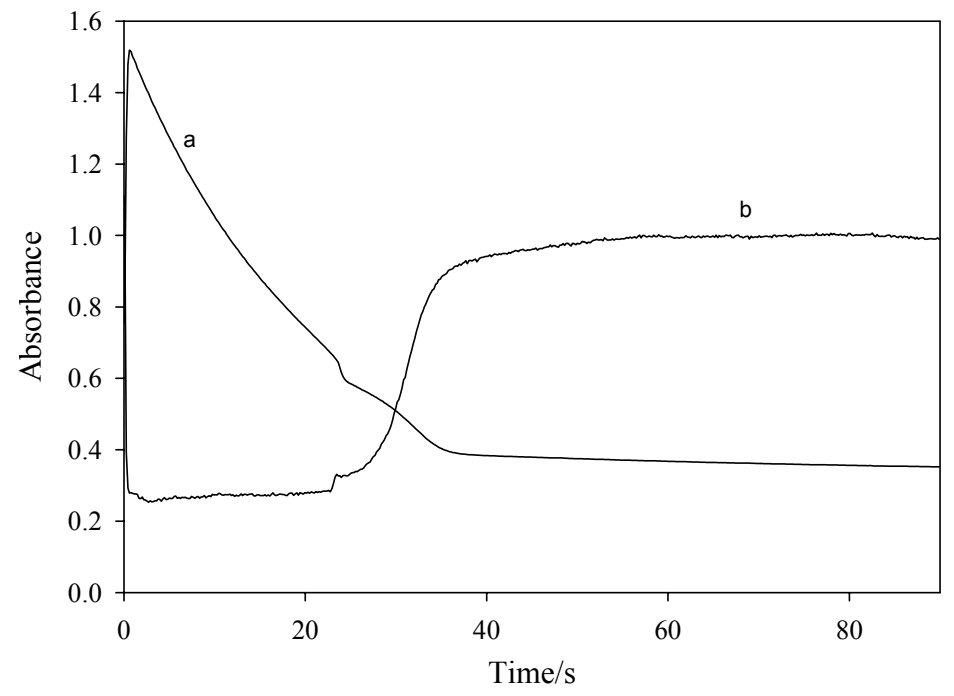

Figure 5.10: Two traces superimposed at (a) $249 \mathrm{~nm}$ and (b) $360 \mathrm{~nm}$. $[\mathrm{PTU}]_{0}=1.25 \times 10^{-4} \mathrm{M}$, $\left[\mathrm{ClO}_{2}^{-}\right]_{0}=7.0 \times 10^{-4} \mathrm{M}$ and $\left[\mathrm{HClO}_{4}\right]_{0}=0.015 \mathrm{M}$

\subsection{MECHANISM}

\subsubsection{Chlorite-PTU reactions}

We are assuming a non-radical pathway in which the initial stages of the reaction involve the formation of the reactive oxyhalogen species, $\mathrm{HOCl}$ : 


$$
\mathrm{ClO}_{2}^{-}+\mathrm{PhN}(\mathrm{H}) \mathrm{CSNH}_{2} \rightarrow \mathrm{PhN}=\mathrm{CS}(\mathrm{OH}) \mathrm{NH}_{2}+\mathrm{OCl}^{-} ; k_{1}
$$

followed by

$$
\mathrm{OCl}^{-}+\mathrm{H}^{+} \rightleftharpoons \mathrm{HOCl} ; K_{\mathrm{a} 2}
$$

$\mathrm{PhN}=\mathrm{CS}(\mathrm{OH}) \mathrm{NH}_{2}$ represents the unstable sulfenic acid which can either be oxidized further to a sulfinic acid, $\mathrm{PhN}=\mathrm{CS}\left(\mathrm{O}_{2} \mathrm{H}\right) \mathrm{NH}_{2}$ (5.8) [29], or can combine with another PTU molecule to form a stable dimer (5.9).

$$
\begin{array}{ll}
\mathrm{ClO}_{2}{ }^{-}+\mathrm{PhN}=\mathrm{CS}(\mathrm{OH}) \mathrm{NH}_{2} \rightarrow \mathrm{PhN}=\mathrm{CS}\left(\mathrm{O}_{2} \mathrm{H}\right) \mathrm{NH}_{2}+\mathrm{OCl}^{-} & 5.8 \\
\mathrm{PhN}=\mathrm{CS}(\mathrm{OH}) \mathrm{NH}_{2}+\mathrm{PhN}(\mathrm{H}) \mathrm{CSNH}_{2} \rightarrow\left(\mathrm{PhN}=\mathrm{C}\left(\mathrm{NH}_{2}\right)-\mathrm{S}\right)_{2}+\mathrm{H}_{2} \mathrm{O} & 5.9
\end{array}
$$

The sulfenic acid can also disproportionate into the more stable PTU and the sulfinic acid:

$$
2 \mathrm{PhN}=\mathrm{CS}(\mathrm{OH}) \mathrm{NH}_{2} \rightleftharpoons \mathrm{PhN}=\mathrm{CS}\left(\mathrm{O}_{2} \mathrm{H}\right) \mathrm{NH}_{2}+\mathrm{PhN}(\mathrm{H}) \mathrm{CSNH}_{2}
$$

Literature has several reports on the formation of thiosulfinates from sulfenic acids in the absence of further oxidant [30]. After the initiation reaction 5.6, the sulfenic acid can only react through reactions $5.8-5.10$. There seems to be no other fate for the sulfenic acid. In excess oxidant, the favored pathway is 5.8. In limited amounts of oxidant (e.g. 1:1 mixture ratios of PTU with an equivalent of a 2-electron oxidant), quantitative dimer formation can be obtained as in reaction 5.9. A bulky dimer, of the type formed in reaction 5.9, however, should be unstable and because of its low solubility should 
precipitate from solution. No precipitate was observed in our experimental observations, thus precluding reaction 5.9 as a major route in this mechanism. We expect, then, that upon depletion of the oxidant, reactions of 5.10-type will dominate such that the favored stable forms of the sulfur compound are formed: PTU itself, the sulfinic acid or sulfate. In our proposed mechanism, the rate-determining step in the oxidation of PTU by chlorite is reaction 5.6. Reaction 5.6, as well as any other reactions that involve the reduction of a chlorine center coupled to the oxidation of a sulfur center, will be considered irreversible in this mechanism. Reaction 5.7 is a rapid protolytic step such that 5.6 and 5.7 can be combined as one reaction even though we recognize that 5.11 is not a termolecular process:

$$
\mathrm{ClO}_{2}^{-}+\mathrm{PhN}(\mathrm{H}) \mathrm{CSNH}_{2}+\mathrm{H}^{+} \rightarrow \mathrm{PhN}=\mathrm{CS}(\mathrm{OH}) \mathrm{NH}_{2}+\mathrm{HOCl}
$$

Reaction 5.11 is an initial electrophilic attack on PTU followed by protonation and elimination of $\mathrm{HOCl}$. Our acid dependence data can be explained by the effectiveness of the direct reaction of chlorous acid with PTU:

$$
\mathrm{HClO}_{2}+\mathrm{PhN}(\mathrm{H}) \mathrm{CSNH}_{2} \rightarrow \mathrm{PhN}=\mathrm{CS}(\mathrm{OH}) \mathrm{NH}_{2}+\mathrm{HOCl} ; k_{2}
$$

The complex acid dependence data obtained can be explained on the basis of protonation equilibria for both chlorous acid and PTU:

$$
\mathrm{ClO}_{2}^{-}+\mathrm{H}^{+} \rightleftharpoons \mathrm{HClO}_{2} ; K_{\mathrm{a} 1}{ }^{-1}
$$




$$
\mathrm{PhN}(\mathrm{H}) \mathrm{CSNH}_{2}+\mathrm{H}^{+} \rightleftharpoons\left[\mathrm{PhN}(\mathrm{H}) \mathrm{CS}(\mathrm{H}) \mathrm{NH}_{2}\right]^{+} ; K_{\mathrm{b}}
$$

In reaction 5.13, $K_{\mathrm{a} 1}$ is the acid dissociation constant for chlorous acid. Our kinetics data have suggested that, initially, the rate of depletion of PTU is first order in both PTU and chlorite with a complex order with respect to acid. If it is assumed that $\mathrm{HClO}_{2}$ is relatively inert (route 5.12 negligible), then the rate of reaction can be derived in terms of chlorite and PTU. The total $\mathrm{Cl}(\mathrm{III})$ species in solution are partitioned between $\mathrm{ClO}_{2}^{-}$and $\mathrm{HClO}_{2}$ according to the following mass balance equation:

$$
[\mathrm{Cl}(\mathrm{III})]_{\mathrm{T}}=\left[\mathrm{ClO}_{2}^{-}\right]+\left[\mathrm{HClO}_{2}\right]
$$

From the following relationship:

$$
\left[\mathrm{HClO}_{2}\right]=\frac{\left[\mathrm{ClO}_{2}^{-}\right]\left[\mathrm{H}^{+}\right]}{K_{\mathrm{a} 1}}
$$

chlorite concentrations can be written in terms of the total chlorine(III) species:

$$
\left[\mathrm{ClO}_{2}^{-}\right]=\frac{K_{\mathrm{a} 1}[\mathrm{Cl}(\mathrm{III})]_{\mathrm{T}}}{K_{\mathrm{a} 1}+\left[\mathrm{H}^{+}\right]}
$$

The derived rate of reaction with 5.11 as the single and rate-determining step will be given by equation 5.18 :

$$
\text { Rate }=-\frac{\mathrm{d}[\mathrm{PTU}]}{\mathrm{dt}}=k_{1} \frac{[\mathrm{PTU}][\mathrm{Cl}(\mathrm{III})]_{\mathrm{T}}\left[H^{+}\right]}{\left(1+K_{\mathrm{a} 1}{ }^{-1}\left[\mathrm{H}^{+}\right]\right)}
$$


Our experimental data do not support equation 5.18 for all the $\mathrm{pH}$ ranges studied. Equation 5.18 suggests retardation when $\left[\mathrm{H}^{+}\right] \geq K_{\mathrm{a} 1}$; as the term with acid concentration in the denominator will dominate. The equation also suggests that the inhibitory effect of acid will diminish at low acid concentrations, $\mathrm{pH}>\mathrm{pK}_{\mathrm{a} 1}$; but does not support acid catalysis in any $\mathrm{pH}$ range. The experimentally observed acid catalysis effect may have its genesis from the oxychlorine reactions that produce the reactive species. Our data would suggest, then, that reaction 5.12, in which the protonated chlorous acid reacts directly with PTU, contributes significantly to the overall reaction rate observed, and is, in fact, the dominant pathway. $K_{\mathrm{a} 1}$ has been reported [31] to be approximately 0.0191 $\mathrm{M}^{-1} \mathrm{~s}^{-1}$. One would expect saturation in acid effect with no further retardation in conditions of $\mathrm{pH}$ lower than 2.0 ; which represents the approximate $\mathrm{pK}_{\mathrm{a}}$ range of chlorous acid. The rate of reaction can now be derived using both reactions 5.6 and 5.12 to obtain the following rate law:

$$
\text { Rate }=-\frac{\mathrm{d}[\mathrm{PTU}]}{\mathrm{dt}}=\frac{[\mathrm{Cl}(\mathrm{III})]_{\mathrm{T}}[\mathrm{PTU}]_{0}}{K_{\mathrm{a} 1}+\left[\mathrm{H}^{+}\right]}\left[k_{1} K_{\mathrm{a} 1}+k_{2}\left[\mathrm{H}^{+}\right]\right]
$$

\subsubsection{Evaluation of kinetic parameters $k_{1}$ and $k_{2}$}

The important kinetic parameters in this reaction system are the rate constants for the reaction between $\mathrm{PTU}$ and $\mathrm{ClO}_{2}^{-}\left(k_{1}\right), \mathrm{HClO}_{2}\left(k_{2}\right)$, and with $\mathrm{ClO}_{2 \text { (aq) }}$. The reaction's acid dependence, on its own, was too complex to be utilized for the evaluation of $k_{1}$ and $k_{2}$. Two complementary experimental techniques had to be used for evaluating $k_{1}$ and $k_{2}$. The values obtained in these two methods could then be checked against each other. Equation 5.19 was utilized for this evaluation by running a series of experiments at fixed 
acid concentrations and collecting initial rates of consumption of PTU at $249 \mathrm{~nm}$. By running a series of experiments at varying combinations of $[\mathrm{PTU}]_{0}$ and $\left[\mathrm{ClO}_{2}^{-}\right]_{0}$ a plot could be made of initial rate of reaction vs. the product of $[\mathrm{PTU}]_{0}$ and $[\mathrm{Cl}(\mathrm{III})]_{\mathrm{T}}$. This plot was preferred over others (e.g. a $[\mathrm{PTU}]_{0}$ dependence plot or $\left[\mathrm{ClO}_{2}^{-}\right]_{0}$ dependence plot) because it gave the origin as a firm data point. In our statistical and graphical analysis, we could then weight the origin of such a plot, and thus greatly reduce experimentally-generated errors. The slope of the linear part of this plot gave the value of the term in the brackets of equation 5.19; but not individual values of $k_{1}$ and $k_{2}$. Next, using as a rough estimate, figure $5.8 \mathrm{c}$, trace c (for example) was utilized to evaluate $k_{2}$ in the limit of high acid where only the second term in equation 5.19 was relevant. This value was brought back into equation 5.19 as the initial value to then fit the initial rate data and evaluate unambiguous values of $k_{1}$ and $k_{2}$, with their relevant error ranges. The second complementary set of experiments involved the use of low acid concentrations in which both $\mathrm{HClO}_{2}$ and $\mathrm{ClO}_{2}^{-}$species were relevant. Three acid concentrations were used: $0.001 \mathrm{M}, 0.003 \mathrm{M}$ and $0.005 \mathrm{M}$. For each of these acid concentrations, six experiments were performed at specific chlorite and PTU concentrations and initial rates were derived and recorded. Six plots of initial rate vs. acid were generated and $k_{1}$ 's (intercept) and $k_{2}$ 's (slope) were evaluated. These values were then refined by the use of the more accurate data set from the first series of experiments. The final values adopted were from this (first) set. This very extensive analysis gave $k_{1}=31.5 \pm 2.3 \mathrm{M}^{-1} \mathrm{~s}^{-1}$ and $k_{2}$ $=114 \pm 7.0 \mathrm{M}^{-1} \mathrm{~s}^{-1}$. The fact that $k_{2}>k_{1}$ was to be expected since an electrophilic attack by chlorite on the nucleophilic sulfur atom of PTU will not be as facile as that by $\mathrm{HClO}_{2}$. The fact that acid was catalytic at low acid concentrations establishes this fact. 
Equation 5.19 supports a saturation of the initial rate as acid is further increased past the $\mathrm{p} K_{\mathrm{a}}$ of chlorous acid. Our experimental observations show a retardation of the rate as acid concentrations are increased past $0.10 \mathrm{M}$. To accommodate this retardation, equation 5.19 has to be modified for these high acid environments. Under these conditions, the $\mathrm{Cl}(\mathrm{III})$ species will exist predominantly as $\mathrm{HClO}_{2}$ with negligible amounts of unprotonated chlorite available. Any variations in rate can no longer be derived solely from the oxychlorine species. The protonation of thiocarbonyl compounds, like PTU, is well known (reaction 5.14) [32]. The nucleophilic sulfur center is easily protonated at low $\mathrm{pH}$ conditions. One would then expect substantial protonation of PTU at the conditions of $\left[\mathrm{H}^{+}\right]>0.10 \mathrm{M}$. We can assume that the protonated PTU species is inert and combine pathways 5.6, 5.12 and the retarding effect of protonation of PTU, 5.14, to give an overall rate of reaction:

$$
\text { Rate }=-\frac{\mathrm{d}[\mathrm{PTU}]}{\mathrm{dt}}=\frac{[\mathrm{Cl}(\mathrm{III})]_{\mathrm{T}}[\mathrm{PTU}]_{0}}{\left(1+K_{\mathrm{b}}\left[\mathrm{H}^{+}\right]\right)\left(K_{\mathrm{a} 1}+\left[\mathrm{H}^{+}\right]\right)}\left[k_{1} K_{\mathrm{a} 1}+k_{2}\left[\mathrm{H}^{+}\right]\right]
$$

Equation 5.20 assumes negligible reaction between chlorous acid and protonated phenylthiourea. A more complete rate law would include the reaction between chlorite anion and the protonated phenylthiourea, but this pathway would be negligible in this scheme because high $\mathrm{pH}$ conditions would increase $\left[\mathrm{ClO}_{2}^{-}\right]$while decreasing the protonated thiol concentration, while low $\mathrm{pH}$ conditions would decrease $\left[\mathrm{ClO}_{2}^{-}\right]$while increasing the protonated thiocarbonyl. Equation 5.20 would deliver the observed complex acid dependence behavior of initial acid catalysis followed by saturation and then retardation. In highly acidic conditions, equation 5.20 can be utilized with very little 
loss in precision, without the first term in the square bracket and the second bracketed term in the denominator which involves reaction by chlorite. This simplification of Equation 5.20 was used in the evaluation of $k_{2}$ for data shown in Figure $5.8 \mathrm{c}$ where it was assumed $[\mathrm{Cl}(\mathrm{III})]=\left[\mathrm{HClO}_{2}\right]$.

The observed variations of rate with acid concentrations depend on the relative magnitudes of $k_{1}$ and $k_{2}$. Our experimental observations indicate acid catalysis up to approximately $\mathrm{pH} 2.00$ followed by a saturation and retardation as acid concentrations are increased past this point. This would indicate a dominance of $k_{2}$ with further retardation upon saturation of $\left[\mathrm{HClO}_{2}\right]$ being effected by the $\left(1+K_{\mathrm{b}}\left[\mathrm{H}^{+}\right]\right)$term which represents protonation of the thiocarbonyl group. Equations 5.19 and 5.20 predict this trend.

\subsubsection{Formation of chlorine dioxide}

Chlorine dioxide is formed by the one-electron oxidation of chlorite. Two possible oxidizing species in this reaction medium are hypochlorous acid (see reaction 5.5) $[24,23]$ and molecular chlorine $[33,34]$. The standard disproportionation reactions that can produce chlorine dioxide are too slow to be effective on this time scale. The kinetics of the hydrolysis of chlorine, have been studied by temperature-jump spectrophotometry and the kinetics parameters are well known [35]:

$$
\mathrm{Cl}_{2}+\mathrm{H}_{2} \mathrm{O} \rightleftharpoons \mathrm{HOCl}+\mathrm{H}^{+}+\mathrm{Cl}^{-}
$$

This is a rapid equilibrium, and so the oxidation of chlorite by $\mathrm{HOCl}$ (reaction 5.5) or by 
$\mathrm{Cl}_{2}$ (reaction 5.22) should be kinetically indistinguishable [36].

$$
2 \mathrm{ClO}_{2}^{-}+\mathrm{Cl}_{2} \rightarrow 2 \mathrm{ClO}_{2}(\mathrm{aq})+2 \mathrm{Cl}^{-}
$$

The mechanism of reaction 5.22 has been reported to proceed through an intermediate that involves an adduct of $\mathrm{ClO}_{2}{ }^{\circ} \mathrm{Cl}_{2}^{-}$which can then eliminate a $\mathrm{Cl}^{-}$ion to give the asymmetric intermediate which then finally gives chlorine dioxide through reaction 5.24 [37]. The mechanism of the oxidation of chlorite by $\mathrm{HOCl}$, is also well known. Taube and Dodgen [36], using isotopic labeling, have concluded that this oxidation proceeds through the asymmetric intermediate $\mathrm{Cl}_{2} \mathrm{O}_{2}[23,34,33,38]$ :

$$
\mathrm{ClO}_{2}{ }^{-}+\mathrm{HOCl}+\mathrm{H}^{+} \rightleftharpoons \mathrm{Cl}_{2} \mathrm{O}_{2}+\mathrm{H}_{2} \mathrm{O}
$$

Further reaction of the intermediate will yield $\mathrm{ClO}_{2}$ :

$$
\mathrm{ClO}_{2}{ }^{-}+\mathrm{Cl}_{2} \mathrm{O}_{2} \rightarrow 2 \mathrm{ClO}_{2}+\mathrm{Cl}^{-}
$$

Both 5.23 and 5.24 are not elementary single step reactions, although the kinetics of these reactions can be derived from their molecularities.

It does appear that the rate of formation of $\mathrm{HOCl}$ from the reduction of $\mathrm{ClO}_{2}^{-}$will determine the rate of formation of $\mathrm{ClO}_{2}$. Composite reaction $5.11(5.6+5.7)$ is the only source of $\mathrm{HOCl}$ at the beginning of the reaction. As the reaction proceeds, the reaction of chlorite with sulfenic acid (produced in reaction 5.11) and other sulfur oxo-acids that are 
not oxidatively saturated will also yield $\mathrm{HOCl}$.

\subsubsection{Consumption of chlorine dioxide}

Figure 5.5 shows that there is a delicate balance between the reactions that form chlorine dioxide and those that consume it. After eliminating 5.22, we can assume that the sole reaction responsible for the formation of chlorine dioxide is reaction 5.5. Chlorine dioxide can be consumed by any of the reducing sulfur species in solution, but, due to its radical nature (unpaired electron) the most significant route, is its direct reaction with PTU. Our kinetics data suggest first order dependence in PTU and chlorine dioxide. The sigmoidal decay kinetics of chlorine dioxide oxidations (figures $5.6 \mathrm{a}-\mathrm{c}$ ) suggest that the initial stages of the reaction involve the formation of the reactive species which then carries the bulk of the oxidation. This appears, in highly acidic conditions, to be $\mathrm{HClO}_{2}$ which will ultimately produce $\mathrm{HOCl}$. The radical chlorine center in chlorine dioxide is electron deficient with a strong formal positive charge while the sulfur center in PTU is electron rich. An equilibrium is set up between chlorine dioxide and PTU by formation of a loose adduct held together by a weak coordinate bond between the chlorine and sulfur centers $[28,21,39]$ :

$$
\mathrm{ClO}_{2}+\mathrm{S}=\mathrm{C}\left(\mathrm{NH}_{2}\right) \mathrm{N}(\mathrm{H}) \mathrm{Ph} \rightleftharpoons\left[\mathrm{O}_{2} \mathrm{Cl}-\mathrm{S}=\mathrm{C}\left(\mathrm{NH}_{2}\right) \mathrm{N}(\mathrm{H}) \mathrm{Ph}\right]
$$

This adduct, can either return to the original reactants, or react further with another chlorine dioxide molecule to form chlorous acid and the sulfenic acid after a hydrolysis: 


$$
\left[\mathrm{O}_{2} \mathrm{Cl}-\mathrm{S}=\mathrm{C}\left(\mathrm{NH}_{2}\right) \mathrm{N}(\mathrm{H}) \mathrm{Ph}\right]+\mathrm{ClO}_{2(\text { aq })} \rightarrow \mathrm{PhN}=\mathrm{CS}(\mathrm{OH}) \mathrm{NH}_{2}+2 \mathrm{HClO}_{2}
$$

The rate-determining step is the formation of the adduct with 5.26 being relatively facile, irreversible, and entropy-driven. The irreversibility of 5.26 is supported by the reaction's inhibition by acid (see figures $5.9 \mathrm{~b}$ and $5.9 \mathrm{c}$ ). Acid inhibition also proves that 5.25 is the rate-determining step since protonation of PTU should inhibit formation of the adduct by forming a poorer nucleophile, $\left[\mathrm{PhN}(\mathrm{H}) \mathrm{CS}(\mathrm{H}) \mathrm{NH}_{2}\right]^{+}$. If the protonated PTU is inert, then the rate of oxidation of PTU by chlorine dioxide will be given by:

$$
-\frac{\mathrm{d}[\mathrm{PTU}]}{\mathrm{dt}}=\frac{\mathrm{k}_{3}[\mathrm{PTU}]\left[\mathrm{ClO}_{2}\right]}{1+K_{\mathrm{b}}\left[\mathrm{H}^{+}\right]}
$$

In the limit of low acid concentrations, $k_{3}$ was evaluated from data of the type shown in figures 5.6 and 5.9. The value obtained of $k_{3}=74 \pm 22 \mathrm{M}^{-1} \mathrm{~s}^{-1}$ was not very reliable due to uncertainties brought about by the sigmoidal decay-type kinetics as well as the uncertainty in the estimation of $K_{\mathrm{b}}$.

\subsubsection{Nonlinear consumption and formation of chlorine dioxide}

Data in figures 5.6a, 5.6b and 5.6c display biphasic kinetics with an initially sigmoidal consumption of chlorine dioxide followed by a slower monotonic decay. While autocatalysis in chlorine dioxide formation has been rationalized through the formation of $\mathrm{HOCl}$, this, alone cannot explain the biphasic kinetics. Table 5.1 shows an analysis of the data in figure 5.6b. The changeover from rapid autocatalytic kinetics occurred after the almost quantitative formation of the sulfinic acid of PTU: 
This suggests that chlorine dioxide is inert to phenylthiourea sulfinic acid or that this reaction is very slow. This was also proved by the observation that chlorine dioxide reacts very slowly with phenylthiourea sulfinic acid (refer to Section 2.3.2.1 for the preparation of phenylthiourea sulfinic acid) to the point of almost being inert. The accuracy of figure $5.6 \mathrm{~b}$ is derived from the fact that these experimental observations are being performed at $360 \mathrm{~nm}$. Since neither PTU nor phenylurea absorb at $360 \mathrm{~nm}$, our experiment can follow the consumption of chlorine dioxide almost exactly. The formation of the phenylthiourea sulfinic acid at the transition between sigmoidal kinetics and a normal decay is thus not in dispute. Figure 5.10 also confirms our assertion: chlorine dioxide is formed as soon as the sulfinic acid is formed. The observed rapid formation of $\mathrm{ClO}_{2}$ in figure 5.4 can also be explained similarly: The final observed rate of formation of $\mathrm{ClO}_{2}$ is from a combination of the reactions that form $\mathrm{ClO}_{2}$ (reaction 5.5), and those that consume it (reactions of type $5.25+5.26$ ). Formation of the sulfinic acid, however, decreases the rate of consumption of $\mathrm{ClO}_{2(\mathrm{aq})}$, and hence the observed sudden increase in the formation of $\mathrm{ClO}_{2(\mathrm{aq})}$. An examination of figure 5.10 confirms this analysis. The formation of the sulfinic acid, which is (relatively) inert to $\mathrm{ClO}_{2}$, makes reactions that form $\mathrm{ClO}_{2}$ dominant over those that consume it, and hence the instant formation of $\mathrm{ClO}_{2}$ upon commencement of the second phase of the reaction. Consumption of the sulfinic acid will then proceed predominantly through oxidation by $\mathrm{HClO}_{2}$ and $\mathrm{HOCl}$ and not by $\mathrm{ClO}_{2}$. Chlorine dioxide's selective inertness is well known. In a previous submission from our laboratories, it was noted that the reaction of chlorite 
and formaldehyde delivered almost quantitative formation of chlorine dioxide based on stoichiometry 5.2 due to the inertness of chlorine dioxide to formaldehyde and formic acid [40].

\subsubsection{Formation of phenylurea}

Further oxidation of the phenylthiourea dioxide (intermediate species) will yield the sulfonic acid which can further be oxidized to yield phenylurea:

$$
\begin{aligned}
& \mathrm{PhN}=\mathrm{CS}\left(\mathrm{O}_{2} \mathrm{H}\right) \mathrm{NH}_{2}+\mathrm{HOCl} \rightarrow \mathrm{PhN}=\mathrm{CS}\left(\mathrm{O}_{3} \mathrm{H}\right) \mathrm{NH}_{2}+\mathrm{H}^{+}+\mathrm{Cl}^{-} \\
& \mathrm{PhN}=\mathrm{CS}\left(\mathrm{O}_{3} \mathrm{H}\right) \mathrm{NH}_{2}+\mathrm{HOCl} \rightarrow \mathrm{PhN}(\mathrm{H}) \mathrm{CONH}_{2}+\mathrm{SO}_{4}{ }^{2-}+3 \mathrm{H}^{+}+\mathrm{Cl}^{-}
\end{aligned}
$$

The accumulation of the sulfinic acid as an intermediate indicates that reactions 5.29 and 5.30 must be slower than reactions 5.6, 5.8 and 5.12. Work by Makarov et al [41] and Otoikhian et al [42] proved that the oxidation of sulfinic and sulfonic acids predominantly goes through the initial cleavage of the C-S bond to give sulfoxylate anion and bisulfite respectively:

$$
\begin{aligned}
& \mathrm{PhN}=\mathrm{CS}\left(\mathrm{O}_{2} \mathrm{H}\right) \mathrm{NH}_{2}+\mathrm{H}_{2} \mathrm{O} \rightarrow \mathrm{PhN}(\mathrm{H}) \mathrm{CONH}_{2}+\mathrm{HSO}_{2}^{-}+\mathrm{H}^{+} \\
& \mathrm{PhN}=\mathrm{CS}\left(\mathrm{O}_{3} \mathrm{H}\right) \mathrm{NH}_{2}+\mathrm{H}_{2} \mathrm{O} \rightarrow \mathrm{PhN}(\mathrm{H}) \mathrm{CONH}_{2}+\mathrm{HSO}_{3}{ }^{-}+\mathrm{H}^{+}
\end{aligned}
$$

Further oxidation of the sulfur leaving groups should be rapid and nearly diffusioncontrolled. These sulfur oxo-acids exist in the form of resonance-stabilized zwitterions that are much more stable in acid than in base [43,41]. This explains why the 
intermediate, sulfinic acid, is slower to react to form products in high acid environments, but quickly gives products in low acid conditions (see figure 5.8c). The cleavage of the $\mathrm{C}-\mathrm{S}$ bond in sulfinic and sulfonic acids in low acid environments is the basis for guanidylation [44].

\subsection{COMPUTER SIMULATIONS}

\subsubsection{Overall reaction network}

The chlorite-PTU and chlorine dioxide-PTU reactions can both be comprehensively explained by a single reaction network of 27 reactions which are shown in Table 5.2. Using relevant assumptions, these reactions can be severely truncated into only a few reactions for high acid concentrations, where chlorite oxidations are deleted in favor of those by chlorous acid. The direct reaction between chlorine dioxide and PTU can be derived by utilizing relevant reactions in Table 5.2 and deleting the rest. The tabulated mechanism is divided into five distinct sections. Reactions M1 - M3 represent the rapid protolytic reactions that are responsible for the observed complex acid dependence. Reaction M3 is relevant at much higher acid concentrations rather than reactions M1 and M2. Reactions M4 - M11 are reactions that involve oxidations by $\mathrm{ClO}_{2}{ }^{-}$and $\mathrm{HClO}_{2}$. The whole reaction scheme is controlled by reactions M4 and M5

which represent the rate-determining steps. The set of reactions M12 - M15 are the autocatalytic steps which also include the formation of chlorine dioxide. M13 is the only reaction through which chlorine dioxide is formed throughout the whole mechanism, and in conditions of excess oxidant, its rate of formation will depend upon the rate of formation of $\mathrm{HOCl}$ from the reduction of $\mathrm{Cl}(\mathrm{III})$ species. 
Table 5.2: The Chlorite-Chlorine Dioxide-Phenylthiourea Reaction Network

\begin{tabular}{|c|c|c|}
\hline & Reaction & $\mathrm{k}_{\mathrm{f}} ; \mathrm{k}_{\mathrm{r}}$ \\
\hline M1 & $\mathrm{ClO}_{2}^{-}+\mathrm{H}^{+} \rightleftharpoons \mathrm{HClO}_{2}$ & $1.0 \times 10^{9} ; 1.91 \times 10^{7}$ \\
\hline M2 & $\mathrm{OCl}^{-}+\mathrm{H}^{+} \rightleftharpoons \mathrm{HOCl}$ & $1.0 \times 10^{9} ; 3.24 \times 10^{1}$ \\
\hline M3 & $\mathrm{PhN}(\mathrm{H}) \mathrm{CSNH}_{2}+\mathrm{H}^{+} \rightleftharpoons\left[\mathrm{PhN}(\mathrm{H}) \mathrm{CS}(\mathrm{H}) \mathrm{NH}_{2}\right]^{+}$ & $1.0 \times 10^{9} ; 1.12 \times 10^{6}$ \\
\hline M4 & $\mathrm{ClO}_{2}^{-}+\mathrm{PhN}(\mathrm{H}) \mathrm{CSNH}_{2} \rightarrow \mathrm{PhN}=\mathrm{CS}(\mathrm{OH}) \mathrm{NH}_{2}+\mathrm{OCl}^{-}$ & 32 \\
\hline M5 & $\mathrm{HClO}_{2}+\mathrm{PhN}(\mathrm{H}) \mathrm{CSNH}_{2} \rightarrow \mathrm{PhN}=\mathrm{CS}(\mathrm{OH}) \mathrm{NH}_{2}+\mathrm{HOCl}$ & 115 \\
\hline M6 & $\mathrm{ClO}_{2}^{-}+\mathrm{PhN}=\mathrm{CS}(\mathrm{OH}) \mathrm{NH}_{2} \rightarrow \mathrm{PhN}=\mathrm{CS}\left(\mathrm{O}_{2} \mathrm{H}\right) \mathrm{NH}_{2}+\mathrm{OCl}^{-}$ & $5.00 \times 10^{3}$ \\
\hline M7 & $\mathrm{ClO}_{2}^{-}+\mathrm{PhN}=\mathrm{CS}\left(\mathrm{O}_{2} \mathrm{H}\right) \mathrm{NH}_{2} \rightarrow \mathrm{PhN}=\mathrm{CS}\left(\mathrm{O}_{3} \mathrm{H}\right) \mathrm{NH}_{2}+\mathrm{OCl}^{-}$ & 95 \\
\hline M8 & $\begin{array}{l}\mathrm{ClO}_{2}^{-}+\mathrm{PhN}=\mathrm{CS}\left(\mathrm{O}_{3} \mathrm{H}\right) \mathrm{NH}_{2} \rightarrow \mathrm{PhN}(\mathrm{H}) \mathrm{CONH}_{2}+\mathrm{SO}_{4}^{2-}+\mathrm{Cl}^{-}+ \\
2 \mathrm{H}^{+}\end{array}$ & 15 \\
\hline M9 & $\mathrm{HClO}_{2}+\mathrm{PhN}=\mathrm{CS}(\mathrm{OH}) \mathrm{NH}_{2} \rightarrow \mathrm{PhN}=\mathrm{CS}\left(\mathrm{O}_{2} \mathrm{H}\right) \mathrm{NH}_{2}+\mathrm{HOCl}$ & $1.00 \times 10^{3}$ \\
\hline M10 & $\mathrm{HClO}_{2}+\mathrm{PhN}=\mathrm{CS}\left(\mathrm{O}_{2} \mathrm{H}\right) \mathrm{NH}_{2} \rightarrow \mathrm{PhN}=\mathrm{CS}\left(\mathrm{O}_{3} \mathrm{H}\right) \mathrm{NH}_{2}+\mathrm{HOCl}$ & 45 \\
\hline M11 & $\begin{array}{l}\mathrm{HClO}_{2} \\
3 \mathrm{H}^{+}\end{array}$ & 12 \\
\hline M12 & $\mathrm{ClO}_{2}^{-}+\mathrm{HOCl}+\mathrm{H}^{+} \rightleftharpoons \mathrm{Cl}_{2} \mathrm{O}_{2}+\mathrm{H}_{2} \mathrm{O}$ & $\begin{array}{l}1.01 \times 10^{6} ; 0.1 \\
1.50 \times 10^{3} ; 5.50 \times 10^{-6}\end{array}$ \\
\hline M13 & $\mathrm{Cl}_{2} \mathrm{O}_{2}+\mathrm{ClO}_{2}^{-} \rightarrow 2 \mathrm{ClO}_{2}+\mathrm{Cl}^{-}$ & \\
\hline M14 & $\mathrm{Cl}_{2} \mathrm{O}_{2}+\mathrm{PhN}(\mathrm{H}) \mathrm{CSNH}_{2}+\mathrm{H}_{2} \mathrm{O} \rightarrow \mathrm{PhN}=\mathrm{CS}(\mathrm{OH}) \mathrm{NH}_{2}+2 \mathrm{HOCl}$ & $2.00 \times 10^{3}$ \\
\hline M15 & $\begin{array}{l}\mathrm{Cl}_{2} \mathrm{O}_{2}+\mathrm{PhN}=\mathrm{CS}(\mathrm{OH}) \mathrm{NH}_{2}+\mathrm{H}_{2} \mathrm{O} \rightarrow \mathrm{PhN}=\mathrm{CS}\left(\mathrm{O}_{2} \mathrm{H}\right) \mathrm{NH}_{2}+ \\
2 \mathrm{HOCl}\end{array}$ & $1.00 \times 10^{3}$ \\
\hline M16 & $\mathrm{HOCl}+\mathrm{PhN}(\mathrm{H}) \mathrm{CSNH}_{2} \rightarrow \mathrm{PhN}=\mathrm{CS}(\mathrm{OH}) \mathrm{NH}_{2}+\mathrm{H}^{+}+\mathrm{Cl}^{-}$ & $5.00 \times 10^{3}$ \\
\hline M17 & $\mathrm{HOCl}+\mathrm{PhN}=\mathrm{CS}(\mathrm{OH}) \mathrm{NH}_{2} \rightarrow \mathrm{PhN}=\mathrm{CS}\left(\mathrm{O}_{2} \mathrm{H}\right) \mathrm{NH}_{2}+\mathrm{H}^{+}+\mathrm{Cl}^{-}$ & $2.00 \times 10^{3}$ \\
\hline M18 & $\mathrm{HOCl}+\mathrm{PhN}=\mathrm{CS}\left(\mathrm{O}_{2} \mathrm{H}\right) \mathrm{NH}_{2} \rightarrow \mathrm{PhN}=\mathrm{CS}\left(\mathrm{O}_{3} \mathrm{H}\right) \mathrm{NH}_{2}+\mathrm{H}^{+}+\mathrm{Cl}^{-}$ & 100 \\
\hline M19 & $\mathrm{HOCl}+\mathrm{PhN}=\mathrm{CS}\left(\mathrm{O}_{3} \mathrm{H}\right) \mathrm{NH}_{2} \rightarrow \mathrm{PhN}(\mathrm{H}) \mathrm{CONH}_{2}+\mathrm{SO}_{4}^{2-}+\mathrm{H}^{+}+\mathrm{Cl}^{-}$ & 50 \\
\hline M20 & $\mathrm{ClO}_{2}+\mathrm{S}=\mathrm{C}\left(\mathrm{NH}_{2}\right) \mathrm{N}(\mathrm{H}) \mathrm{Ph} \rightleftharpoons\left[\mathrm{O}_{2} \mathrm{Cl}-\mathrm{S}=\mathrm{C}\left(\mathrm{NH}_{2}\right) \mathrm{N}(\mathrm{H}) \mathrm{Ph}\right]$ & 74 \\
\hline
\end{tabular}




\begin{tabular}{|c|c|c|}
\hline M21 & $\begin{array}{l}{\left[\mathrm{O}_{2} \mathrm{Cl}-\mathrm{S}=\mathrm{C}\left(\mathrm{NH}_{2}\right) \mathrm{N}(\mathrm{H}) \mathrm{Ph}\right]+\mathrm{ClO}_{2}(\mathrm{aq}) \rightarrow \mathrm{PhN}=\mathrm{CS}(\mathrm{OH}) \mathrm{NH}_{2}+} \\
2 \mathrm{HClO}_{2}\end{array}$ & $1.00 \times 10^{3}$ \\
\hline M22 & $\mathrm{ClO}_{2}+\mathrm{HOS}-\mathrm{C}\left(\mathrm{NH}_{2}\right)=\mathrm{NPh} \rightleftharpoons\left[\mathrm{O}_{2} \mathrm{Cl}-(\mathrm{HO}) \mathrm{S}-\mathrm{C}\left(\mathrm{NH}_{2}\right)=\mathrm{NPh}\right.$ & 50 \\
\hline M23 & {$\left[\mathrm{O}_{2} \mathrm{Cl}-(\mathrm{HO}) \mathrm{S}-\mathrm{C}\left(\mathrm{NH}_{2}\right)=\mathrm{NPh} \rightarrow \mathrm{PhN}=\mathrm{CS}\left(\mathrm{O}_{2} \mathrm{H}\right) \mathrm{NH}_{2}+2 \mathrm{HClO}_{2}\right.$} & $1.00 \times 10^{3}$ \\
\hline M24 & $\mathrm{PhN}=\mathrm{CS}\left(\mathrm{O}_{2} \mathrm{H}\right) \mathrm{NH}_{2}+\mathrm{H}_{2} \mathrm{O} \rightarrow \mathrm{PhN}(\mathrm{H}) \mathrm{CONH}_{2}+\mathrm{HSO}_{2}^{-}+\mathrm{H}^{+}$ & 500 \\
\hline M25 & $\mathrm{PhN}=\mathrm{CS}\left(\mathrm{O}_{3} \mathrm{H}\right) \mathrm{NH}_{2}+\mathrm{H}_{2} \mathrm{O} \rightarrow \mathrm{PhN}(\mathrm{H}) \mathrm{CONH}_{2}+\mathrm{HSO}_{3}^{-}+\mathrm{H}^{+}$ & 50 \\
\hline M26 & $\mathrm{HSO}_{2}^{-}+\mathrm{HOCl} \rightarrow \mathrm{HSO}_{3}^{-}+\mathrm{H}^{+}+\mathrm{Cl}^{-}$ & $5.00 \times 10^{4}$ \\
\hline M27 & $\mathrm{HSO}_{3}^{-}+\mathrm{HOCl} \rightarrow \mathrm{SO}_{4}^{2-}+2 \mathrm{H}^{+}+\mathrm{Cl}^{-}$ & $1.00 \times 10^{4}$ \\
\hline M28 & $2 \mathrm{ClO}_{2}+\mathrm{HSO}_{2}^{-}+\mathrm{H}_{2} \mathrm{O} \rightarrow \mathrm{HSO}_{3}^{-}+2 \mathrm{HClO}_{2}$ & $4.00 \times 10^{6}$ \\
\hline M29 & $2 \mathrm{ClO}_{2}+\mathrm{HSO}_{3}^{-}+\mathrm{H}_{2} \mathrm{O} \rightarrow \mathrm{SO}_{4}^{2-}+\mathrm{H}^{+}+2 \mathrm{HClO}_{2}$ & $5.00 \times 10^{5}$ \\
\hline
\end{tabular}

Legend:

$\mathrm{PhN}=\mathrm{CS}(\mathrm{OH}) \mathrm{NH}_{2}-$ phenylthiourea sulfenic acid $\mathrm{PhN}=\mathrm{CS}\left(\mathrm{O}_{2} \mathrm{H}\right) \mathrm{NH}_{2}-$ phenylthiourea sulfinic acid $\mathrm{PhN}=\mathrm{CS}\left(\mathrm{O}_{3} \mathrm{H}\right) \mathrm{NH}_{2}-$ phenylthiourea sulfonic acid

Rate constants - forward and reverse rate constants (column 3) separated by a semi-colon.

Note: Reactions M8, M11 and M19 all require a water molecule (solvent) for material balance Units of rate constants are derived from the reaction's molecularity except where water is involved, and also in M28 and M29 which are composites of two consecutive steps and considered bimolecular.

Reactions M16 - M19 represent the dominant oxidation pathway. The autocatalytic production of $\mathrm{ClO}_{2}$ transfers the bulk of oxidation to $\mathrm{HOCl}$, even though, at the beginning of the reaction, before the build-up of $\mathrm{HOCl}$ concentrations, the major oxidation pathway would have been the series of reactions M4 - M11. Reactions M20 - M23 involve oxidation by $\mathrm{ClO}_{2}$. From the basis of our experimental observations, no reactions have been included that involve oxidation of the sulfinic and sulfonic acids by $\mathrm{ClO}_{2}$. The final set of four reactions, M24 - M27 represent the hydrolysis of the sulfinic and sulfonic acids. While recent experimental data indicates that these oxo-acids are more stable in acidic environments $[41,43]$, this mechanism could not handle stabilization by acid. 
Further mechanistic studies need to be performed before a rate law for the decomposition of these oxo-acids can be derived.

\subsubsection{Choice and establishment of rate constants}

The first three reactions, being rapid and protolytic could be assigned any rate constants as long as they were fast enough so as not to be rate-determining and not violate the $\mathrm{p} K_{\mathrm{a}}$ 's of $\mathrm{HClO}_{2}$ and $\mathrm{HOCl}$ as well as the $\mathrm{p} K_{\mathrm{b}}$ of the thiocarbamide. Since $\mathrm{p} K_{\mathrm{a}}$ of chlorous acid is $1.72[23,45]$; the forward and reverse rate constants utilized had to satisfy $K_{\mathrm{a}}=0.0191$. Literature reports $\mathrm{p} K_{\mathrm{a}}$ of $\mathrm{HOCl}$ as 7.49 [23,31]; and so this was also utilized in the same manner as for $\mathrm{HClO}_{2} . K_{\mathrm{b}}$ for the thiocarbamide was difficult to evaluate, but a value of $8.9 \times 10^{2} \mathrm{M}^{-1}$ was derived for a similar thiocarbamide: guanylthiourea [32]. Protonation of PTU, however, was only relevant in very high acid environments, while most of our work was performed in the intermediate acid concentration ranges where $K_{\mathrm{b}}$ was not relevant. In fact for most of our simulations, this reaction was shut down with no loss of accuracy.

Rate constants for M4 and M5 were derived from this study. Reactions M6 and M9 were all made faster than reactions M4 and M5 respectively, and this was a reasonable assumption after setting M4 and M5 as rate-determining. Reactions M7, M8 M10 and M11 were guessed for best fit, but could be ignored, reactions M24 and M25 were made labile enough. M12 and M13 were derived from literature values. Since rate of reaction was controlled by rate of formation of $\mathrm{HOCl}$, reactions M16 and M17 were set such that they were faster than reactions M4 and M5. Kinetics parameters for reaction M20 were derived from this study, and subsequently, $k_{\mathrm{M} 22}$ was made slightly faster than $k_{\mathrm{M} 20}$. 
Reactions M26 and M27 are known to be fast and nearly diffusion controlled, and were implemented accordingly. The autocatalytic reactions M14 and M15 were only relevant in low to medium acid concentrations. They were very important in determining the sigmoidal decay kinetics observed in figure $5.7 \mathrm{c}$.

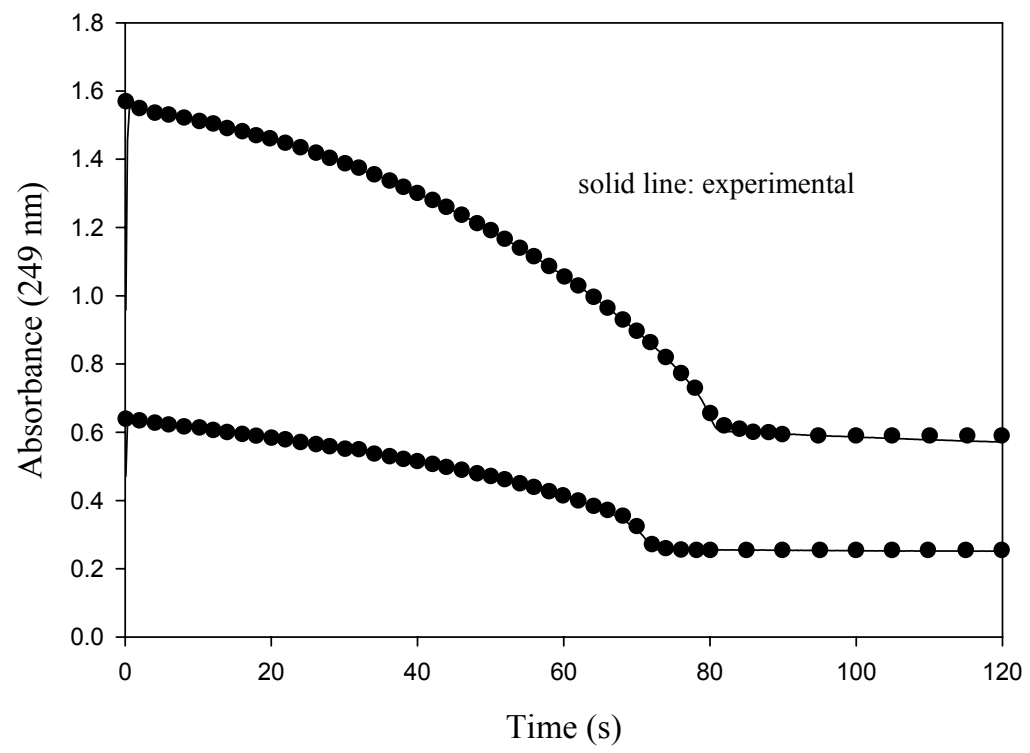

Figure 11: Simulations of the data shown in Figure 7c (traces b and e) using mechanism in Table 2.

This model could adequately simulate figures $5.2 \mathrm{a}, 5.3 \mathrm{~b}, 5.6 \mathrm{a}, 5.7 \mathrm{c}, 5.9 \mathrm{a}$ and $5.9 \mathrm{~b}$. While it could simulate the first part of figure $5.8 \mathrm{c}$, the uncertainties in the hydrolysis kinetics of the sulfinic and sulfonic acids made it difficult to simulate to second phase of that reaction. Figure 5.11 shows as simulation fit to the data shown in figure $5.7 \mathrm{c}$. These simulations showed that reactions M28 and M29 are extremely important in accelerating the rate of consumption of PTU by rapidly forming $\mathrm{ClO}_{2}{ }^{-}$from $\mathrm{ClO}_{2}$. 


\subsection{CONCLUSION}

The oxidation of phenylthiourea is complicated by the formation of two stable intermediates: the sulfinic acid and the sulfonic acid on the pathway towards total desulfurization to form phenylurea. The reaction is extremely complex with reaction dynamics strongly influenced by the $\mathrm{pH}$ of the reaction medium. Its standard use as a goitrogenic must lie in its ability to abstract the iodine atom needed by the thyroid gland [12]. Its toxicity, however, must be derived from the deliberate cleavage of the C-S bond to yield highly reactive sulfur species that may interact with oxygen to yield reactive oxygen species. This appears to be a well-accepted route for the establishment of toxicities in thiocarbamides $[46,47]$. The physiological environment, which is slightly basic, destabilizes the oxo-acids in favor of the reduced sulfur species after cleavage of the C-S bond $[41,46]$. 


\section{References}

1. P. E. J. Wheatcroft and C. C. Thornburn, "Toxicity of the taste testing compound phenylthiocarbamide," Nature 235, 93-94 (1972).

2. S. Wooding, U. k. Kim, M. J. Bamshad, J. Larsen, L. B. Jorde, and D. Drayna, "Natural selection and molecular evolution in PTC, a bitter-taste receptor gene," Am. J. Hum. Genet. 74, 637-646 (2004).

3. J. Cohen and D. P. Ogdon, "Taste blindness to phenylthiocarbamide and related compounds," Psychol. Bull. 46, 490-498 (1949).

4. J. Cohen and D. P. Ogdon, "Taste blindness to phenylthiocarbamide as a function of saliva," Science 110, 532 (1949).

5. K. Akeo, D. B. Ebenstein, and C. K. Dorey, "Dopa and oxygen inhibit proliferation of retinal pigment epithelial cells, fibroblasts and endothelial cells in vitro," Exp. Eye Res. 49, 335-346 (1989).

6. J. A. Prezioso, G. B. Fitzgerald, and M. M. Wick, "Effects of tyrosinase activity on the cytotoxicity of 3,4-dihydroxybenzylamine and buthionine sulfoximine in human melanoma cells," Pigment Cell Res. 3, 49-54 (1990).

7. J. Karlsson, J. Von Hofsten, and P. E. Olsson, "Generating transparent zebrafish: A refined method to improve detection of gene expression during embryonic development," Mar. Biotechnol. 3, 522-527 (2001).

8. M. E. Morrison, M. J. Yagi, and G. Cohen, "In vitro studies of 2,4dihydroxyphenylalanine, a prodrug targeted against malignant melanoma cells," Proc. Natl. Acad. Sci. USA 82, 2960-2964 (1985).

9. O. A. Elsalini and K. B. Rohr, "Phenylthiourea disrupts thyroid function in developing zebrafish," Dev. Genes Evol. 212, 593-598 (2003).

10. C. S. Lee, E. H. Song, S. Y. Park, and E. S. Han, "Combined effect of dopamine and MPP+ on membrane permeability in mitochondria and cell viability in PC12 cells," Neurochem. Int. 43, 147-154 (2003).

11. W. X. Guo, L. L. Poulsen, and D. M. Ziegler, "Use of thiocarbamides as selective substrate probes for isoforms of flavin-containing monooxygenases," Biochem. Pharmacol. 44, 2029-2037 (1992).

12. F. Facchini, A. Abbati, and S. Campagnoni, "Possible relations between sensitivity to phenylthiocarbamide and goiter," Hum.Biol. 62, 545-552 (1990).

13. R. Bruck, D. Frenkel, H. Shirin, H. Aeed, Z. Matas, M. Papa, L. Zaidel, Y. Avni, R. Oren, and Z. Halpern, "Hypothyroidism protects rat liver from acetaminophen hepatotoxicity," Dig. Dis. Sci. 44, 1228-1235 (1999). 
14. U. Kabadi and R. Cech, "Therapeutic 131I dose in hyperthyroidism: Role of pretreatment with thionamide," Thyroidology 6, 87-92 (1994).

15. A. M. Scott, G. M. Powell, D. G. Upshall, and C. G. Curtis, "Pulmonary toxicity of thioureas in the rat," Environ. Health Perspect. 85, 43-50 (1990).

16. R. L. Smith and R. T. Williams, "The metabolism of arylthioureas. III. (a) The toxicity of hydrogen sulfide in relation to that of phenylthiourea. (b) The protection of rats against the toxic effects of phenylthiourea, with 1-methyl-1-phenylthiourea," J. Med. Pharm. Chem. 4, 137-146 (1961).

17. R. L. Smith and R. T. Williams, "The metabolism of arylthioureas. I. The metabolism of 1,3-diphenyl-2-thiourea (thiocarbanilide) and its derivatives," J. Med. Pharm. Chem. 4, 97-107 (1961).

18. S. H. Dieke, G. S. Allen, and C. P. Richter, "The acute toxicity of thioureas and related compounds to wild and domestic Norway rats," J. Pharmacol. 90, 260-270 (1947).

19. D. M. Ziegler, "Metabolic basis of detoxication: Functional groups bearing sulfur," in Metabolic Basis of Detoxication: Metabolism of Functional Groups, edited by W.B. Jakoby, J.R. Bend, and J. Caldwell, (Academic Press, New York, 1982) p. 171-184.

20. I. R. Epstein, K. Kustin, and R. H. Simoyi, "Systematic design of chemical oscillators. 78. Kinetics and mechanism of the chlorite-thiourea reaction in acidic medium," J. Phys. Chem. 96, 5852-5856 (1992).

21. A. K. Horvath, I. Nagypal, and I. R. Epstein, "Kinetics and mechanism of the chlorine dioxide-tetrathionate reaction," J. Phys. Chem. A 107, 10063-10068 (2003).

22. L. Wang and D. W. Margerum, "Hypohalite ion catalysis of the disproportionation of chlorine dioxide," Inorg. Chem. 41, 6099-6105 (2002).

23. Z. Jia, D. W. Margerum, and J. S. Francisco, "General-acid-catalyzed reactions of hypochlorous acid and acetyl hypochlorite with chlorite ion," Inorg. Chem. 39, 2614-2620 (2000).

24. G. Peintler, I. Nagypal, and I. R. Epstein, "Systematic design of chemical oscillators. 60. Kinetics and mechanism of the reaction between chlorite ion and hypochlorous acid," J. Phys. Chem. 94, 2954-2958 (1990).

25. J. B. Jones, C. R. Chinake, and R. H. Simoyi, "Oxyhalogen-sulfur chemistry: oligooscillations in the formamidinesulfinic acid-chlorite reaction," J. Phys. Chem. 99, 1523-1529 (1995). 
26. M. A. Salem, C. R. Chinake, and R. H. Simoyi, "Nonlinear dynamics in chemistry derived from sulfur chemistry. part 13. oxyhalogen-sulfur chemistry: Oxidation of hydroxymethanesulfinic acid by chlorite," J. Phys. Chem. 100, 9377-9384 (1996).

27. J. N. Figlar and D. M. Stanbury, "Kinetics and a revised mechanism for the autocatalytic oxidation of $\mathrm{SCN}$ by $\mathrm{ClO}_{2}$," J. Phys. Chem. A 103, 5732-5741 (1999).

28. J. Darkwa, R. Olojo, E. Chikwana, and R. H. Simoyi, "Antioxidant chemistry: Oxidation of L-cysteine and its metabolites by chlorite and chlorine dioxide," J. Phys. Chem. A 108, 5576-5587 (2004).

29. J. Darkwa, R. Olojo, O. Olagunju, A. Otoikhian, and R. Simoyi, "Oxyhalogensulfur chemistry: Oxidation of n-acetylcysteine by chlorite and acidic bromate," J. Phys. Chem. A 107, 9834-9845 (2003).

30. G. Capozzi and G. Modena, "Oxidation of Thiols," in The Chemistry of the Thiol Group: Part 2, edited by S.Patai, (J. Wiley and Sons, London, 1974) p. 785-839.

31. E. Hwang, J. N. Cash, and M. J. Zabik, "Chlorine and chlorine dioxide treatment to reduce or remove EBDCs and ETU residues in a solution," J. Agricult. Food Chem. 50, 4734-4742 (2002).

32. E. Chikwana and R. H. Simoyi, "Oxyhalogen-sulfur chemistry: Kinetics and mechanism of oxidation of amidinothiourea by acidified iodate," J. Phys. Chem. A 108, 1024-1032 (2004).

33. C. S. Furman, Z. Jia, and D. W. Margerum, "Mechanisms of chlorine dioxide and chlorate ion formation from the reactions of chlorite ion with hypobromous and hypochlorous acids," Book of Abstracts, 216th ACS National Meeting, p.335 (1998).

34. C. S. Furman and D. W. Margerum, "Mechanism of chlorine dioxide and chlorate ion formation from the reaction of hypobromous acid and chlorite ion," Inorg. Chem. 37, 4321-4327 (1998).

35. M. Eigen and K. Kustin, "Kinetics of halogen hydrolysis," J. Am. Chem. Soc. 84, 1355-1361 (1962).

36. H. Taube and H. Dodgen, "Applications of radioactive chlorine to the study of the mechanisms of reactions involving changes in the oxidation state of chlorine," J. Am. Chem. Soc. 71, 3330-3336 (1949).

37. J. S. Nicoson and D. W. Margerum, "Kinetics and mechanisms of aqueous chlorine reactions with chlorite ion in the presence of chloride ion and acetic acid/acetate buffer," Inorg. Chem. 41, 342-347 (2002).

38. C. R. Chinake, C. Mundoma, R. Olojo, T. Chigwada, and R. H. Simoyi, "Oxyhalogen-sulfur chemistry: Kinetics and mechanism of the oxidation of a Bunte 
salt 2-aminoethanethiolsulfuric acid by chlorite," Phys. Chem. Chem. Phys. 3, 4957-4964 (2001).

39. A. K. Horvath and I. Nagypal, "Kinetics and mechanism of the reaction between thiosulfate and chlorine dioxide," J. Phys. Chem. A 102, 7267-7272 (1998).

40. C. R. Chinake, O. Olojo, and R. H. Simoyi, "Oxidation of formaldehyde by chlorite in basic and slightly acidic media," J. Phys. Chem. A 102, 606-611 (1998).

41. S. V. Makarov, C. Mundoma, J. H. Penn, S. A. Svarovsky, and R. H. Simoyi, "New and surprising experimental results from the oxidation of sulfinic and sulfonic acids," J. Phys. Chem. A 102, 6786-6792 (1998).

42. J. F. Ojo, A. Otoikhian, R. Olojo, and R. H. Simoyi, "Oxyhalogen-sulfur chemistry: Nonlinear oxidation kinetics of hydroxymethanesulfinic acid by acidic iodate," J. Phys. Chem. A 108, 2457-2463 (2004).

43. S. V. Makarov, C. Mundoma, J. H. Penn, J. L. Petersen, S. A. Svarovsky, and R. H. Simoyi, "Structure and stability of aminoiminomethanesulfonic acid," Inorg. Chim. Acta 286, 149-154 (1999).

44. A. K. Datta, X. Shi, and K. S. Kasprzak, "Effect of carnosine, homocarnosine and anserine on hydroxylation of the guanine moiety in 2'-deoxyguanosine, DNA and nucleohistone with hydrogen peroxide in the presence of nickel(II)," Carcinogenesis 14, 417-422 (1993).

45. I. Fabian, D. Szucs, and G. Gordon, "Unexpected phenomena in the mercury(II)chlorite ion system: Formation and kinetic role of the $\mathrm{HgClO}_{2}{ }^{+}$Complex," J. Phys. Chem. A 104, 8045-8049 (2000).

46. S. V. Makarov, C. Mundoma, S. A. Svarovsky, X. Shi, P. M. Gannett, and R. H. Simoyi, "Reactive oxygen species in the aerobic decomposition of sodium hydroxymethanesulfinate," Arch. Biochem. Biophys. 367, 289-296 (1999).

47. S. A. Svarovsky, R. H. Simoyi, and S. V. Makarov, "A possible mechanism for thiourea-based toxicities: kinetics and mechanism of decomposition of thiourea dioxides in alkaline solutions," J. Phys. Chem. B 105, 12634-12643 (2001). 


\section{CHAPTER 6}

\section{Kinetics and Mechanism of the Oxidation of a Bunte salt 2-aminoethanethiolsulfuric acid by chlorite}

\subsection{INTRODUCTION}

This chapter reports on the comprehensive investigations into the oxidation of 2aminoethanethiolsulfuric acid, (AETSA) [1], by chlorite as part of a series of studies aimed at the elucidation of oxidative mechanisms of organosulfur compounds by oxyhalogen ions [2]. Another important reason why this study was done was to learn more about the important amino acid taurine, $\mathrm{H}_{2} \mathrm{NCH}_{2} \mathrm{CH}_{2} \mathrm{SO}_{3} \mathrm{H}$ [3-6]. The Bunte salt, AETSA, is a metabolic precursor of taurine. AETSA is widely used as an antiulcerogenic. The use of AETSA reduced the ulcerogenic effects of phenylbutazone or oxyphenylbutazone when they were used as anti-inflammatory agents [7]. This therapeutic effect of AETSA is often linked to taurine itself.

Taurine one of the most abundant amino acids differs from other amino acids in containing a sulfonic acid group, instead of the requisite carboxylic acid group. It is found in the central nervous system, skeletal muscle and is very concentrated in the brain and heart tissues $[8,9]$. Taurine is involved in a number of physiological processes such as membrane stabilization [10], detoxification [11-17], inhibition and modulation of neurotransmitters in the brain [18], bile acid conjugation and cholesterol excretion [19], osmoregulation [20-23], and regulation of intracellular $\mathrm{Ca}^{2+}$ homeostasis [24,25].

Previous studies have shown the oxidation of AETSA by iodate [26] to be quite complex, while oxidation by bromate [27] was much simpler. Motivation of these studies 
was the need to deduce the conditions under which the $\mathrm{S}-\mathrm{S}$ bond and/or the $\mathrm{C}-\mathrm{S}$ bond in the thiolsulfuric acid cleaved, as well as to determine the products of reaction. A comparison of these two systems (AETSA $/ \mathrm{BrO}_{3}^{-}$and $\left.\mathrm{AETSA} / \mathrm{IO}_{3}{ }^{-}\right)$with the AETSA $/ \mathrm{ClO}_{2}{ }^{-}$can shed more light into the oxidation of AETSA as well as the reaction products.

\subsection{RESULTS}

\subsubsection{Stoichiometry}

In excess chlorite conditions, the stoichiometry of the reaction was determined to be:

$$
2 \mathrm{ClO}_{2}{ }^{-}+\mathrm{H}_{2} \mathrm{NCH}_{2} \mathrm{CH}_{2} \mathrm{~S}-\mathrm{SO}_{3} \mathrm{H} \rightarrow \mathrm{ClNHCH}_{2} \mathrm{CH}_{2} \mathrm{SO}_{3} \mathrm{H}+\mathrm{SO}_{4}{ }^{2-}+\mathrm{Cl}^{-}+\mathrm{H}^{+}
$$

Whereas in excess AETSA, the stoichiometry was found to be:

$$
\begin{aligned}
3 \mathrm{ClO}_{2}^{-}+2 \mathrm{H}_{2} \mathrm{NCH}_{2} \mathrm{CH}_{2} \mathrm{~S}_{-} \mathrm{SO}_{3} \mathrm{H}+2 \mathrm{H}_{2} \mathrm{O} \rightarrow \\
2 \mathrm{NH}_{2} \mathrm{CH}_{2} \mathrm{CH}_{2} \mathrm{SO}_{3} \mathrm{H}+2 \mathrm{SO}_{4}{ }^{2-}+3 \mathrm{Cl}^{-}+4 \mathrm{H}^{+}
\end{aligned}
$$

These stoichiometries were derived spectrophotometrically as well as by iodometric and titrimetric techniques following procedures outlined in chapter 2. Qualitative tests were positive for both $\mathrm{SO}_{4}{ }^{2-}$ and $\mathrm{Cl}^{-}$. Stoichiometry 6.2 was especially correct for reactions carried out under mildly acidic conditions. The stoichiometry obtained was usually between 6.1 and 6.2. NMR data showed that there was evidence for the formation of the dichlorotaurine which could be formed from the disproportionation of monochlorotaurine [28]: 


$$
2 \mathrm{ClNHCH}_{2} \mathrm{CH}_{2} \mathrm{SO}_{3} \mathrm{H} \rightleftharpoons \mathrm{NH}_{2} \mathrm{CH}_{2} \mathrm{CH}_{2} \mathrm{SO}_{3} \mathrm{H}+\mathrm{Cl}_{2} \mathrm{NCH}_{2} \mathrm{CH}_{2} \mathrm{SO}_{3} \mathrm{H}
$$

Under highly acidic conditions the equilibrium (6.3) was shifted to the right, due to protonation of the taurine amino group:

$$
\mathrm{NH}_{2} \mathrm{CH}_{2} \mathrm{CH}_{2} \mathrm{SO}_{3} \mathrm{H}+\mathrm{H}^{+} \rightleftharpoons\left[\mathrm{H}_{3} \mathrm{~N} \mathrm{CH}_{2} \mathrm{CH}_{2} \mathrm{SO}_{3} \mathrm{H}\right]^{+}
$$

UV/VIS and NMR spectrometry techniques identified chlorotaurine, $\mathrm{ClNHCH}_{2} \mathrm{CH}_{2} \mathrm{SO}_{3} \mathrm{H}$, as the major product from the initial oxidation of AETSA.

The reaction mixtures were incubated for a period of 36 hours, after which their NMR spectrum was obtained. Figure 6.1 shows a series of NMR spectra used to determine the nature of the products from the reaction. The spectrum of AETSA in an acidic solution is shown in (a). Only one peak, a multiplet comprising of two overlapping triplets at ca. $\delta=$ 3.26 is observed, this is characteristic of coupling of adjacent methylene protons. Spectrum (b) shows changes to the spectrum after addition of chlorite but before the reaction had gone to completion. The spectrum reveals the emergence of two triplets downfield to the previous multiplet. These triplets can only be formed from a sulfoxide in which the inner sulfur atom picks up an oxygen atom:

$$
\mathrm{H}_{2} \mathrm{NCH}_{2} \mathrm{CH}_{2} \mathrm{~S}-\mathrm{SO}_{3} \mathrm{H}+[\mathrm{O}] \rightarrow \mathrm{H}_{2} \mathrm{NCH}_{2} \mathrm{CH}_{2} \mathrm{~S}(\mathrm{O}) \mathrm{SO}_{3} \mathrm{H}
$$




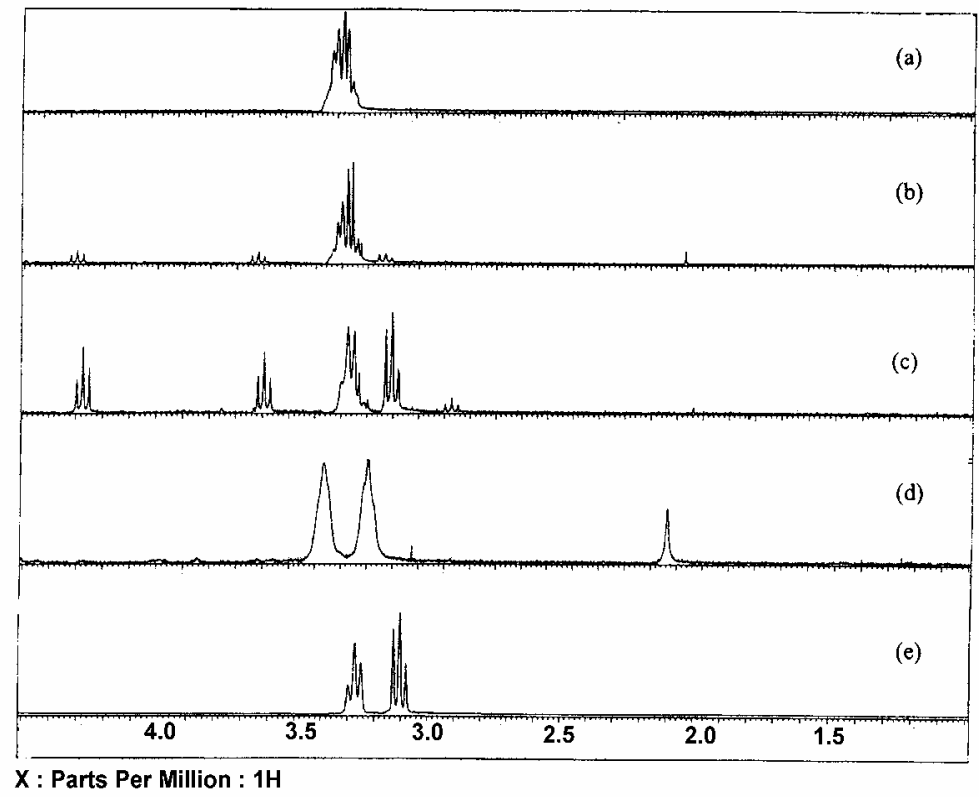

Figure 6.1: (a) NMR spectrum of AETSA in acid (b) Spectrum of reaction products of AETSA $\mathrm{ClO}_{2}{ }^{-}$reaction in acid showing appearance of new peaks after 5 minutes of reaction (c) NMR spectrum of AETSA - $\mathrm{ClO}_{2}{ }^{-}$taken after 24 hours under condition of excess AETSA (d) Spectrum of same reaction under conditions of excess $\mathrm{ClO}_{2}^{-}$after 24 hours incubation period. (e) NMR spectrum of chlorotaurine.

The sulfoxide renders the inner sulfur center asymmetric [26], with the adjacent hydrogen atoms diastereotopic, thus inducing a new set of two triplets downfield:<smiles>NCCCCS(=O)(=O)O</smiles>

Spectrum (c) shows the triplet peaks increasing in height, demonstrating the stability of the sulfoxide as well as an increase in its concentration. At this point, the former AETSA multiplet bifurcates into the distinctive triplet peaks of taurine. Upon oxidation by excess chlorite, the multiplex resolved into two broad peaks at $\delta_{\alpha}=3.21-3.28$ and $\delta_{\beta}=3.37-3.45$, 
which are associated with a series of compounds which all contain the two sets of methylene protons, as shown in spectrum (d). These triplets are not as resolved as the ones for pure taurine shown in spectrum (e). This apparent lack of resolution is due to the mixing of the three taurine-based products: taurine, chlorotaurine and dichlorotaurine. The substitution of chlorine atoms for the hydrogen atoms at the nitrogen center introduces a more electron-withdrawing group, but the effect is not as strong since the chlorine atom attaches to the nitrogen center as a positive cation. The protons attached to nitrogen and oxygen atoms, however, cannot be detected due to rapid exchange with solvent.

\subsubsection{Reaction Dynamics}

The reaction could only be followed by NMR and UV/Visible absorbance measurements. Specific electrodes for chloride and sulfate were quickly poisoned by the sulfur generated from the AETSA that was deposited on the electrode surfaces. The high ionic strengths used precluded the use of conductivity measurements. In conditions of excess chlorite, the reaction could be followed by observing the formation of chlorine dioxide. The chlorine dioxide is formed after some induction period that is determined by the initial concentrations of the reagents, especially the ratio of reductant (AETSA) to

oxidant (chlorite). Formation of chlorine dioxide is very rapid and autocatalytic after the expiration of the induction period. Figure 6.2 showed that at fixed AETSA concentrations, increasing chlorite concentrations decreased the induction period and increased the rate of formation of chlorine dioxide. The sharp and rapid rate of formation of chlorine dioxide after the induction period is very significant and allows us 


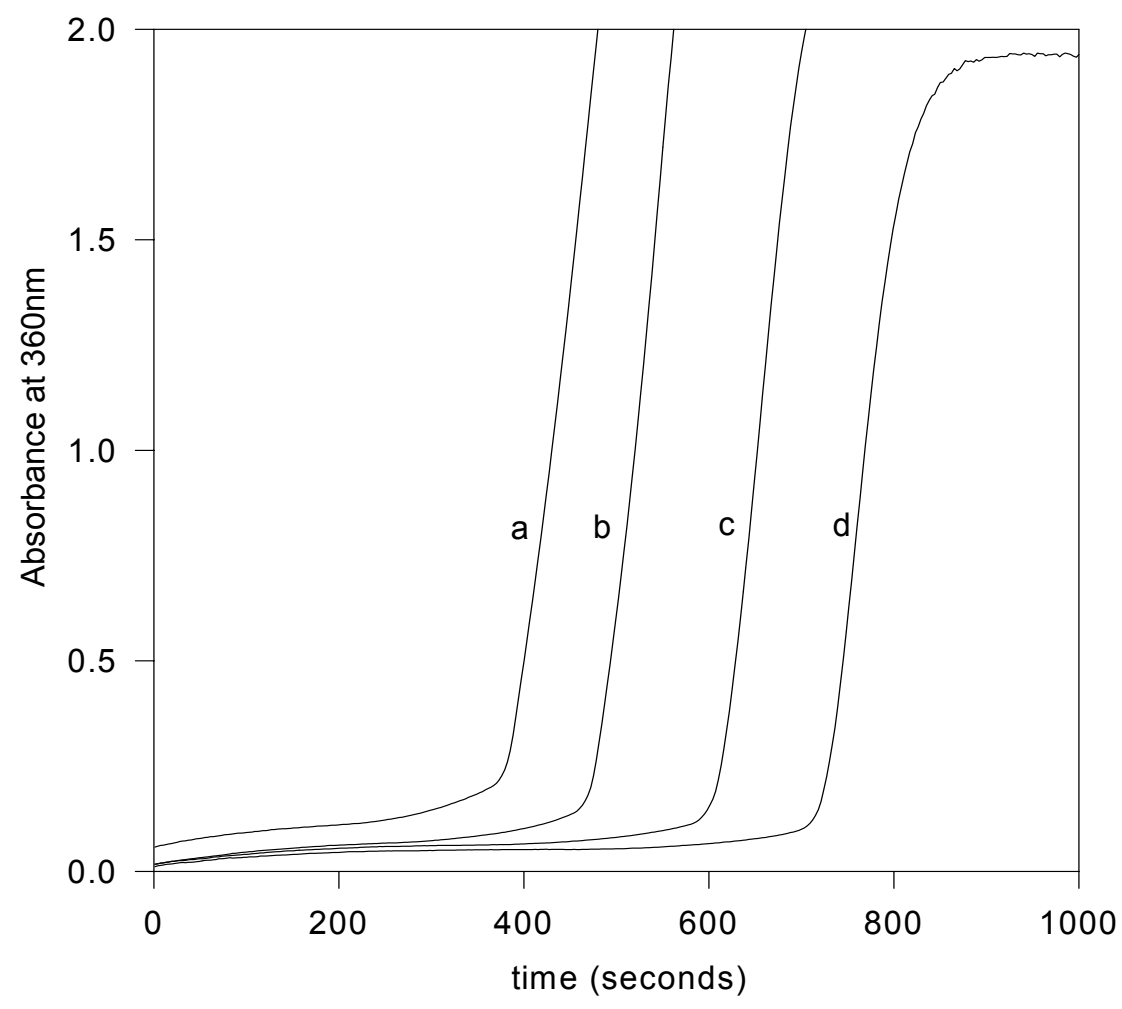

Figure 6.2. Effect of chlorite on the absorbance activity at $360 \mathrm{~nm}$. Concentration of chlorite is directly proportional to the amount of $\mathrm{ClO}_{2}$ formed and inversely proportional to the induction period at fixed AETSA concentration: $[\mathrm{AETSA}]_{\mathrm{o}}=0.001 \mathrm{M},\left[\mathrm{H}^{+}\right]_{\mathrm{o}}=0.05 \mathrm{M}, \mathrm{I}_{(\mathrm{NaCl})}=1.0 \mathrm{M},\left[\mathrm{ClO}_{2}{ }^{-}\right.$ ] = (a) $7.0 \times 10^{-3} \mathrm{M}$, (b) $6.0 \times 10^{-3} \mathrm{M}$, (c) $5.0 \times 10^{-3} \mathrm{M}$, (d) $4.0 \times 10^{-3} \mathrm{M}$.

to separate the reaction into two sections: the initial part of the reaction that involves the consumption of AETSA, and the final part, which involves an extraneous oxyhalogen reaction that forms chlorine dioxide. It would appear, from the absorbance data that the rates of these two parts of the reaction are so different in magnitude that they can be treated as two separate processes. Thus the induction period observed would be related to the rate of reaction that consumes AETSA in an inverse-type of dependence; i.e. a shorter induction period would suggest a more rapid consumption of AETSA. Figure 6.3 shows the plot of the induction time versus the inverse concentration of chlorite. The fact that this plot gives a straight line implies that the reaction that depletes AETSA is first order in chlorite [29]. Figure 6.3 was also important in confirming our stoichiometry. 


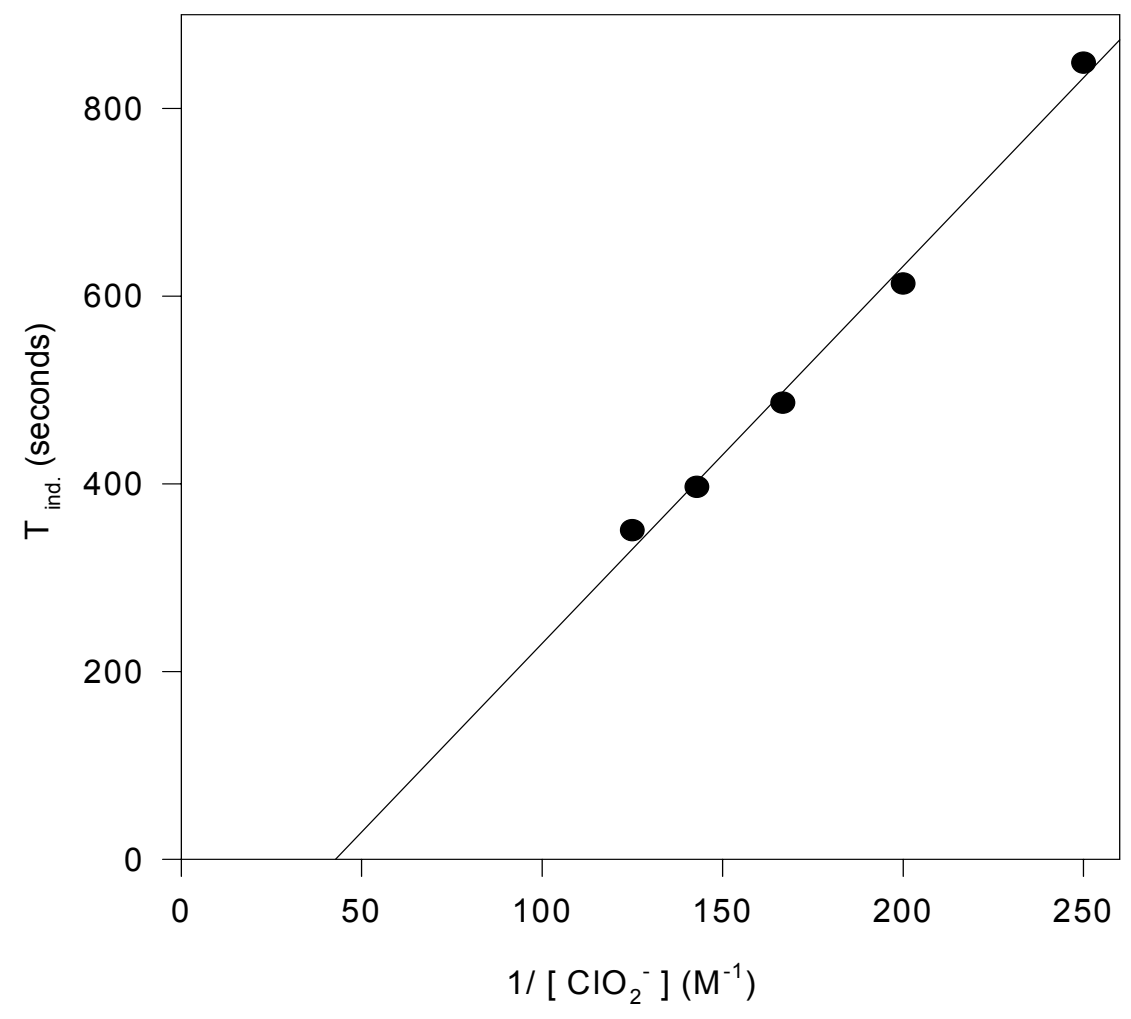

Figure 6.3: Plot of induction time vs. $1 /\left[\mathrm{ClO}_{2}^{-}\right]$using stoichiometric excess of chlorite. The reciprocal of $\mathrm{x}$-intercept corresponds to minimum $\left[\mathrm{ClO}_{2}^{-}\right]$necessary for formation of $\mathrm{ClO}_{2}$. The $\mathrm{y}-$ intercept has no kinetic significance. $[\mathrm{AETSA}]_{\mathrm{o}}=0.001 \mathrm{M},\left[\mathrm{H}^{+}\right]_{\mathrm{o}}=0.05 \mathrm{M}, \mathrm{I}_{(\mathrm{NaCl})}=1.0 \mathrm{M},\left[\mathrm{ClO}_{2}^{-}\right]$ $=$ (a) $7.0 \times 10^{-3} \mathrm{M}$, (b) $6.0 \times 10^{-3} \mathrm{M}$, (c) $5.0 \times 10^{-3} \mathrm{M}$, (d) $4.0 \times 10^{-3} \mathrm{M}$, and (e) $3.0 \times 10^{-3} \mathrm{M}$.

Whenever there is excess chlorite to that needed to just attain stoichiometry 6.1 , then the excess chlorite after consumption of AETSA will react to form $\mathrm{ClO}_{2}$, and one can measure a finite induction period. However, if there is less chlorite to satisfy stoichiometry 6.1 , then there will be no formation of $\mathrm{ClO}_{2}$ and the induction period will be infinity. (This would not be obtained if rate of reaction $\mathrm{ClO}_{2}$ with AETSA was slow). However, the $x$-intercept of the graph gives the inverse of the amount of chlorite just needed to satisfy stoichiometry 6.1. In this reaction the intercept concentration for 0.001 M AETSA was $0.0022 \mathrm{M} \mathrm{ClO}_{2}^{-}$, thus confirming stoichiometry 6.1. The stoichiometry was not exactly 2:1 because of the expected formation of dichlorotaurine. The $y$-intercept however, does not have any stoichiometric significance. 


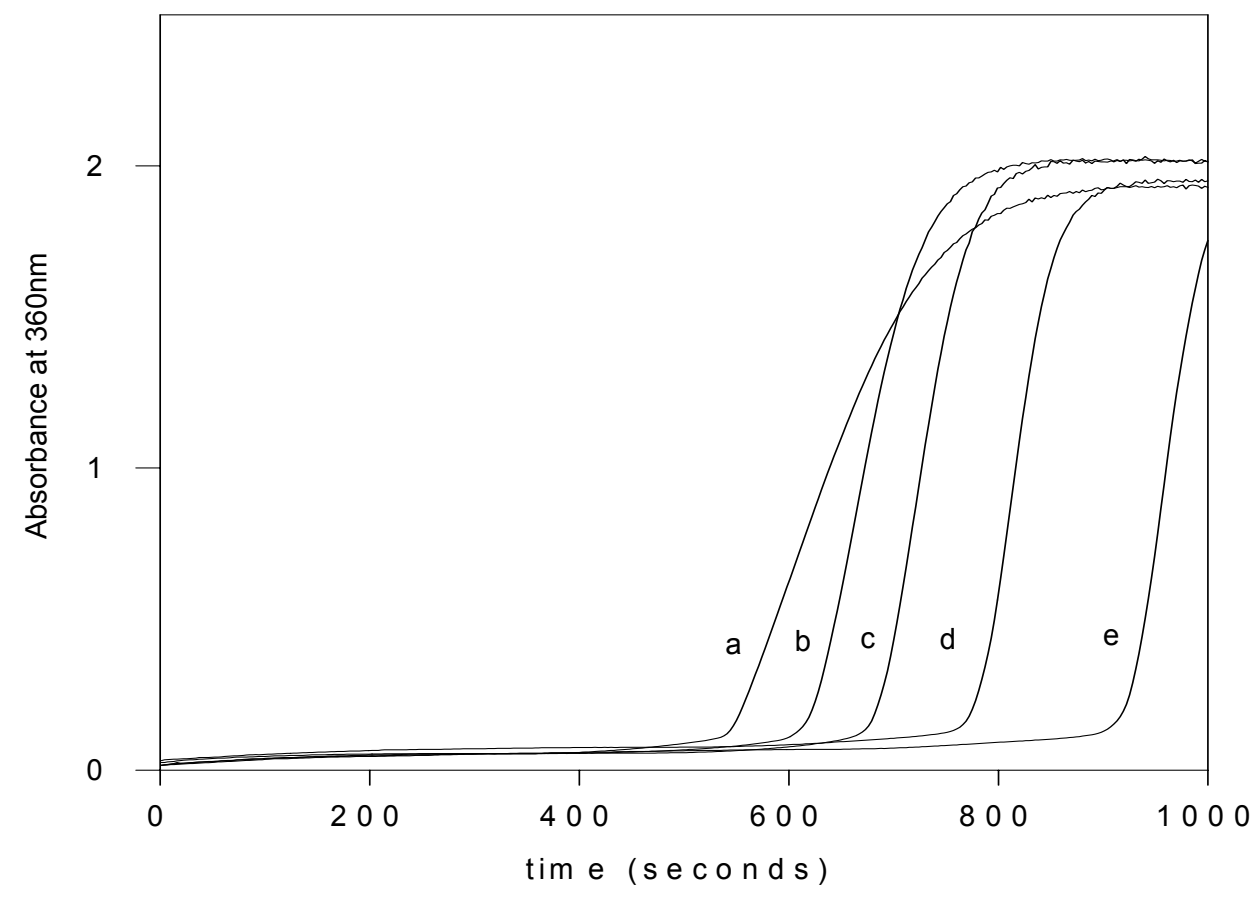

Figure 6.4: Absorbance traces at $360 \mathrm{~nm}$ showing effect of acid on the oxidation of AETSA by chlorite. Induction time for the reaction is inversely dependent on the $\left[\mathrm{H}^{+}\right]_{\mathrm{o}}$. $[\mathrm{AETSA}]_{\mathrm{o}}=1.0 \times 10^{-}$ ${ }^{3} \mathrm{M},\left[\mathrm{ClO}_{2}^{-}\right]=$(a) $4.0 \times 10^{-3} \mathrm{M}, \mathrm{I}_{(\mathrm{NaCl})}=1.0 \mathrm{M},\left[\mathrm{H}^{+}\right]_{\mathrm{o}}=$ (a) $0.08 \mathrm{M}$, (b) $0.06 \mathrm{M}$, (c) $0.05 \mathrm{M}$, (d) $0.04 \mathrm{M}$, (e) $0.025 \mathrm{M}$.

The reaction presented a very complex dependence on acid when compared to chlorite (Figure 6.4). It appears the effect of acid is more pronounced at low acid than at high acid concentrations. Linearity in the plot of inverse acid concentrations versus induction period is lost as the acid concentration increases (Figure 6.5). Figure 6.4 shows that although acid shortens the induction period, the rate of production of chlorine dioxide was retarded by the high acid concentrations. Experiments performed with $\left[\mathrm{ClO}_{2}{ }^{-}\right.$ ]$_{0} \geq[\mathrm{AETSA}]_{0}$, showed an increase in induction period with increasing AETSA concentrations. This is to be expected if the total consumption of AETSA is a prerequisite to the formation of chlorine dioxide (Figure 6.6). In conditions where $\left[\mathrm{ClO}_{2}^{-}\right.$ ]$_{0}>>[\mathrm{AETSA}]_{0}$, the induction period did not change although the rate of formation of chlorine dioxide increased with higher initial AETSA concentrations. 


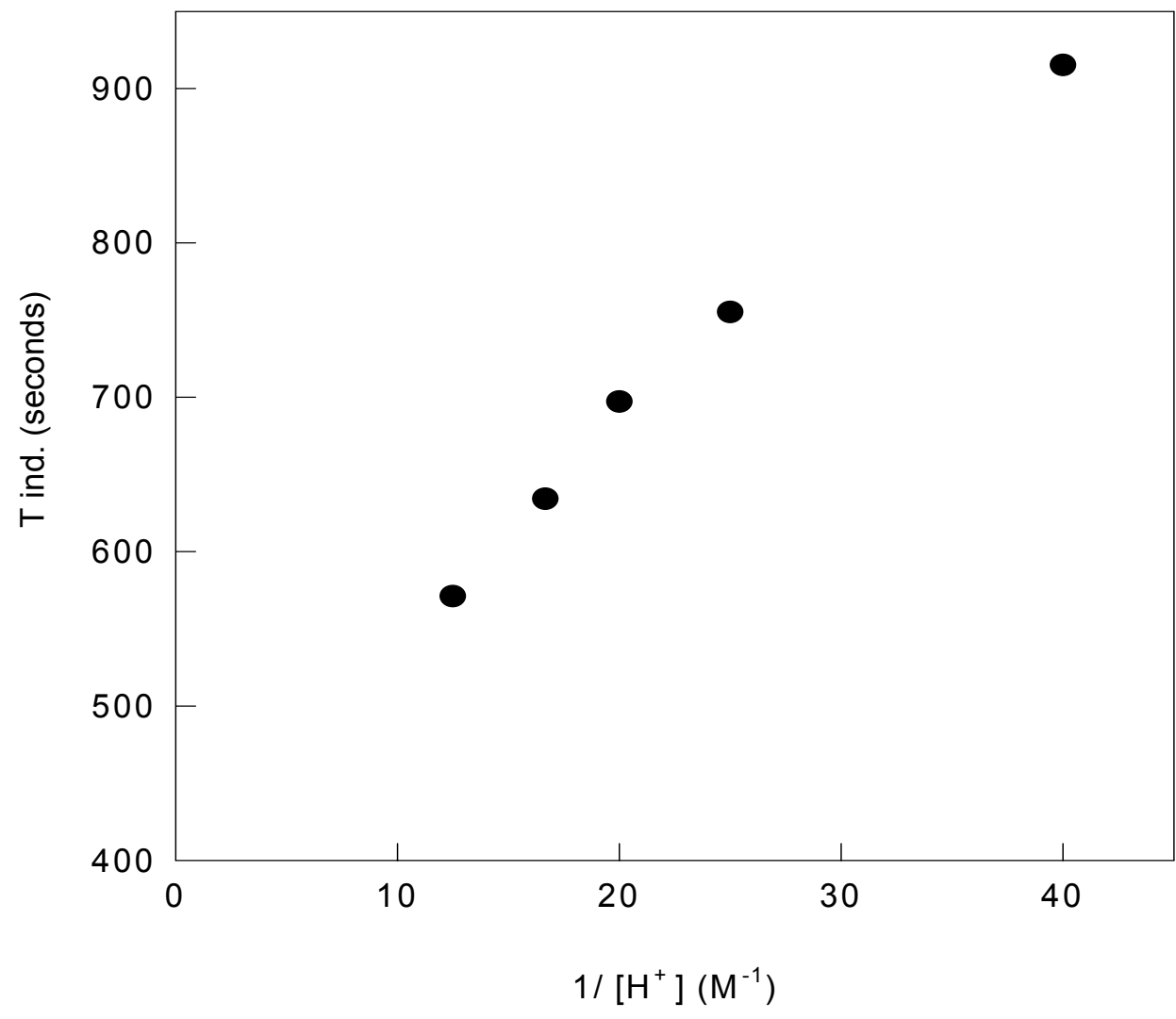

Figure 6.5: Induction time vs. the inverse of the initial acid concentrations $\left[\mathrm{H}^{+}\right]_{\mathrm{o}}$. The induction period is more sensitive to acid at lower concentrations. [AETSA $]_{\mathrm{o}}=1.0 \times 10^{-3} \mathrm{M},\left[\mathrm{ClO}_{2}^{-}\right]=4.0$ x $10^{-3} \mathrm{M}, \mathrm{I}_{(\mathrm{NaCl})}=1.0 \mathrm{M},\left[\mathrm{H}^{+}\right]_{\mathrm{o}}=$ (a) $0.08 \mathrm{M}$, (b) $0.06 \mathrm{M}$, (c) $0.05 \mathrm{M}$, (d) $0.04 \mathrm{M}$, (e) $0.025 \mathrm{M}$.

The rate of reaction between chlorine dioxide and the reducing substrate, AETSA, determines the induction time for the reaction. If the rate of reaction is slow, then we would expect oligooscillatory behavior [30] in the concentration of chlorine dioxide. However, if the rate of reaction between the two is faster than the rate of formation of chlorine dioxide, then a sharp induction period will be observed as no chlorine dioxide will be observed until the reducing substrate, AETSA, has been completely oxidized. 


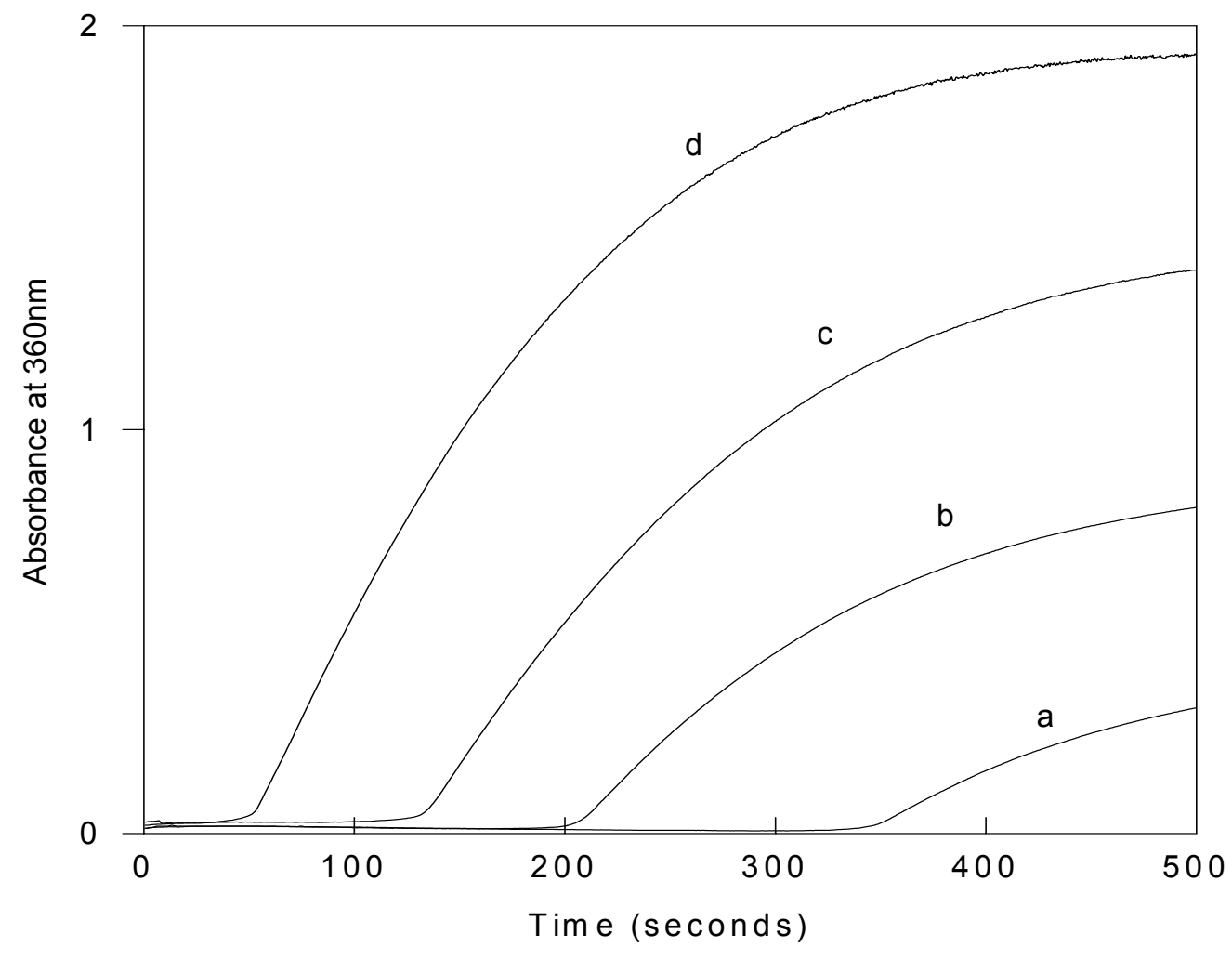

Figure 6.6: Effect of varying $[\mathrm{AETSA}]_{\mathrm{o}}$ on formation of chlorotaurine at fixed chlorite concentration. Traces show that AETSA is completely depleted before the formation of $\mathrm{ClO}_{2}$ commences: $\left[\mathrm{ClO}_{2}^{-}\right]=3.8 \times 10^{-3} \mathrm{M}, \mathrm{I}_{(\mathrm{NaCl})}=1.0 \mathrm{M},\left[\mathrm{H}^{+}\right]_{\mathrm{o}}=0.30 \mathrm{M},[\text { AETSA }]_{\mathrm{o}}=$ (a) $2.0 \times 10^{-3}$, (b) $1.5 \times 10^{-3}$, (c) $1.0 \times 10^{-3} \mathrm{M}$, (d) $5.0 \times 10^{-4} \mathrm{M}$.

Figure 6.7 shows a very complex reaction of chlorine dioxide with AETSA. In excess AETSA, there is a rapid and smooth consumption of chlorine dioxide as can be seen in traces $\mathrm{c}-\mathrm{e}$. In conditions where $\left[\mathrm{ClO}_{2}\right]_{0} \cong[\mathrm{AETSA}]_{0}$ we observed an initial depletion of chlorine dioxide followed by re-formation of chlorine dioxide. 


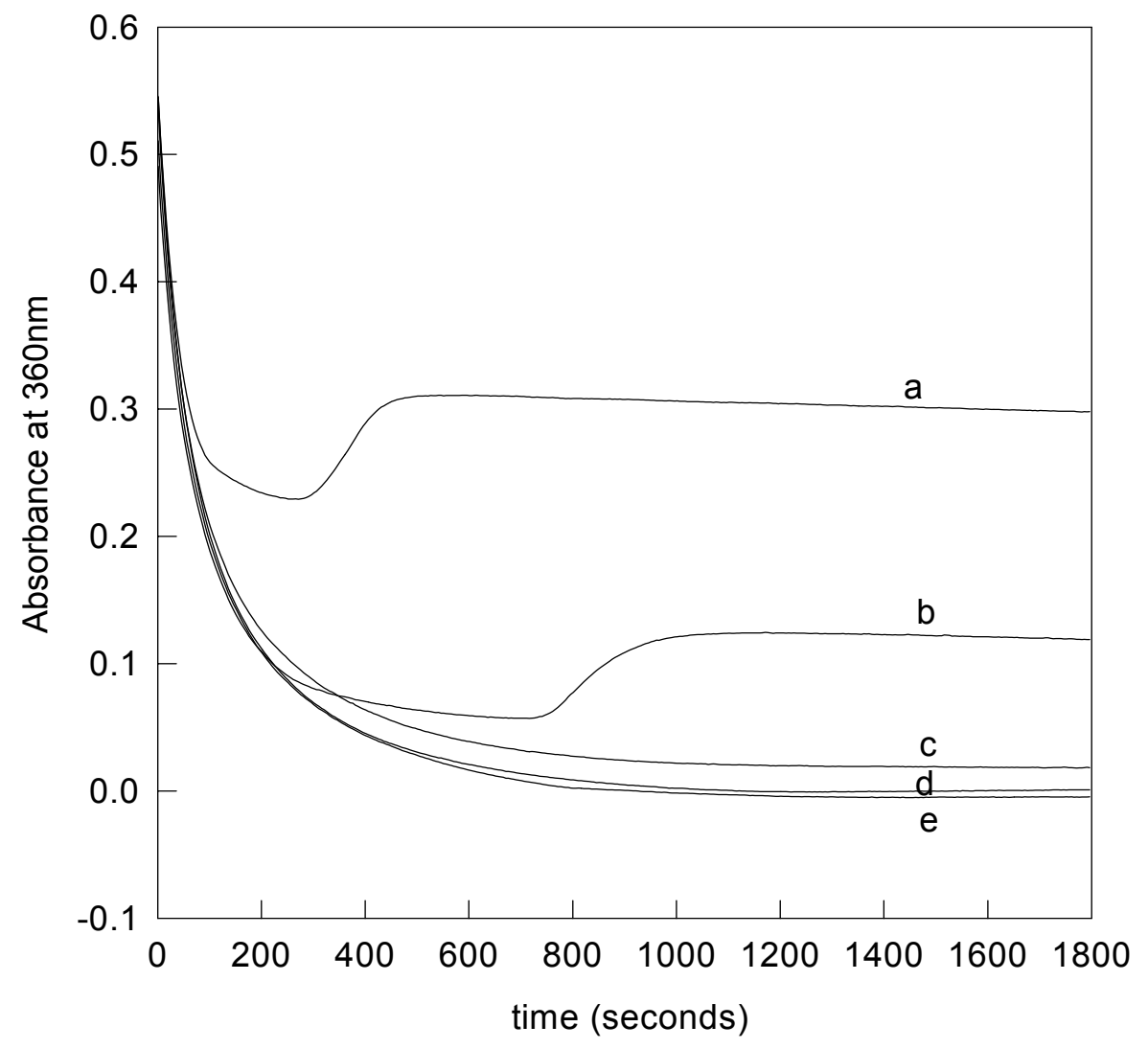

Figure 6.7: Absorbance traces of AETSA oxidation by chlorine dioxide, $\mathrm{ClO}_{2}$ in acidic solution. $\left[\mathrm{ClO}_{2}\right]=6.61 \times 10^{-4} \mathrm{M} ;\left[\mathrm{H}^{+}\right]=0.10 \mathrm{M} ; \mathrm{I}_{(\mathrm{NaCl})}=1.0 \mathrm{M} ;[\mathrm{AETSA}]=(\mathrm{a}) 3.0 \times 10^{-4} \mathrm{M}$ (b) $4.0 \times 10^{-4}$ $\mathrm{M}$ (c) $5.0 \times 10^{-4} \mathrm{M}$ (d) $6.0 \times 10^{-4} \mathrm{M}$ (e) $7.5 \times 10^{-4} \mathrm{M}$

\subsection{MECHANISM}

From the data shown in Figure 6.7, it would appear that the total oxidation of AETSA is a prerequisite for the formation of chlorine dioxide. NMR data suggests a step-wise oxidation that passes through the sulfoxide.

$$
\mathrm{H}_{2} \mathrm{~N}-\mathrm{CH}_{2}-\mathrm{CH}_{2}-\mathrm{S}-\mathrm{SO}_{3} \mathrm{H}+\mathrm{H}_{2} \mathrm{O} \rightarrow \mathrm{H}_{2} \mathrm{~N}-\mathrm{CH}_{2}-\mathrm{CH}_{2}-\mathrm{S}(\mathrm{O})-\mathrm{SO}_{3} \mathrm{H}+2 \mathrm{H}^{+}+2 \mathrm{e}^{-}
$$

The oxidant could be $\mathrm{ClO}_{2}{ }^{-}$or any of its decomposition products: $\mathrm{HOCl}$ and $\mathrm{Cl}_{2}$. 


\subsubsection{Acid Dependence}

The acid dependence data was so complex that it has to be addressed first. Chlorous acid has a $\mathrm{pK}_{\mathrm{a}}$ of about 2.0 [31] and thus will exist predominantly in the protonated form at $\mathrm{pH}$ conditions lower than 2:

$$
\mathrm{ClO}_{2}^{-}+\mathrm{H}^{+} \rightleftharpoons \mathrm{HClO}_{2} ; K_{a 1}
$$

The AETSA should also be protonated, but this will be predominantly on the amino group:

$$
\mathrm{H}_{2} \mathrm{~N}-\mathrm{CH}_{2}-\mathrm{CH}_{2}-\mathrm{S}-\mathrm{SO}_{3} \mathrm{H}+\mathrm{H}^{+} \rightleftharpoons\left[\mathrm{H}_{3} \mathrm{~N}-\mathrm{CH}_{2}-\mathrm{CH}_{2}-\mathrm{S}_{-} \mathrm{SO}_{3} \mathrm{H}\right]^{+}\left(\mathrm{AETSA}-\mathrm{H}^{+}\right)
$$

One can assume complete protonation of the AETSA under conditions utilized in our experiments, and wherever the structure of AETSA is indicated, it will be in the protonated form. There, will, however, be a mixture of $\mathrm{HClO}_{2}$ and $\mathrm{ClO}_{2}{ }^{-}$in solution, with both species reacting with AETSA, but at different rates.

$$
\begin{aligned}
& \mathrm{H}_{2} \mathrm{~N}-\mathrm{CH}_{2}-\mathrm{CH}_{2}-\mathrm{S}-\mathrm{SO}_{3} \mathrm{H}+\mathrm{ClO}_{2}^{-}+\mathrm{H}^{+} \rightarrow \mathrm{H}_{2} \mathrm{~N}-\mathrm{CH}_{2}-\mathrm{CH}_{2}-\mathrm{S}(\mathrm{O})-\mathrm{SO}_{3} \mathrm{H}+\mathrm{HOCl} ; k_{1} \text { (6.9) } \\
& \mathrm{H}_{2} \mathrm{~N}-\mathrm{CH}_{2}-\mathrm{CH}_{2}-\mathrm{S}-\mathrm{SO}_{3} \mathrm{H}+\mathrm{HClO}_{2} \rightarrow \mathrm{H}_{2} \mathrm{~N}-\mathrm{CH}_{2}-\mathrm{CH}_{2}-\mathrm{S}(\mathrm{O})-\mathrm{SO}_{3} \mathrm{H}+\mathrm{HOCl} ; \quad k_{2}
\end{aligned}
$$

It would be safe to assume that reactions 6.9 and 6.10 are rate-determining and hence the rate of consumption of AETSA can be written as: 


$$
\text { Rate }=k_{1}[\mathrm{AETSA}]\left[\mathrm{ClO}_{2}^{-}\right]\left[\mathrm{H}^{+}\right]+k_{2}[\mathrm{AETSA}]\left[\mathrm{HClO}_{2}\right]
$$

Reaction 6.9 is not actually a termolecular process. It involves the initial attack of chlorite on AETSA followed by a very rapid protolytic process that abstracts a proton to yield electroneutrality. Effect of the acid will be determined by the values of $k_{1}$ and $k_{2}$. The following mass balance equation, relates the concentrations of $\mathrm{ClO}_{2}{ }^{-}$and $\mathrm{HClO}_{2}$, and accounts for all Chlorine(III) species in solution:

$$
[\mathrm{Cl}(\mathrm{III})]_{\mathrm{T}}=\left[\mathrm{ClO}_{2}^{-}\right]+\left[\mathrm{HClO}_{2}\right]
$$

The equilibrium in reaction 6.7 allows us to write the following relation as well:

$$
\left[\mathrm{HClO}_{2}\right]=K_{\mathrm{al}}^{-1}\left[\mathrm{ClO}_{2}^{-}\right]\left[\mathrm{H}^{+}\right]
$$

From equations 6.12 and 6.13 , the chlorite and chlorous acid concentrations can be written in terms of the total Chlorine (III) species:

$$
\left[\mathrm{ClO}_{2}{ }^{-}\right]=\frac{[\mathrm{Cl}(\mathrm{III})]_{\mathrm{T}}}{1+K_{\mathrm{al}}^{-1}\left[\mathrm{H}^{+}\right]}
$$

and

$$
\left[\mathrm{HClO}_{2}\right]=\frac{K_{\mathrm{al}}^{-1}\left[\mathrm{H}^{+}\right][\mathrm{Cl}(\mathrm{III})]_{\mathrm{T}}}{1+K_{\mathrm{a} 1}^{-1}\left[\mathrm{H}^{+}\right]}
$$


Using equations 6.14 and 6.15 one can write equation 6.11 in a form that involves only the measurable quantities:

$$
\text { Rate }=[\mathrm{AETSA}][\mathrm{Cl}(\mathrm{III})]_{\mathrm{T}}\left[\mathrm{H}^{+}\right]\left\{\frac{k_{1}}{1+K_{\mathrm{a} 1}^{-1}\left[\mathrm{H}^{+}\right]}+\frac{k_{2} K_{\mathrm{a} 1}^{-1}}{1+K_{\mathrm{a} 1}^{-1}\left[\mathrm{H}^{+}\right]}\right\}
$$

At very low acid concentrations, $1+K_{\mathrm{a} 1}^{-1}\left[\mathrm{H}^{-1}\right] \approx 1$ and equation 6.16 simplifies to:

$$
\text { Rate }=[\mathrm{AETSA}][\mathrm{Cl}(\mathrm{III})]_{\mathrm{T}}\left[\mathrm{H}^{+}\right]\left\{k_{1}+k_{2} K_{\mathrm{a} 1}^{-1}\right\}
$$

Thus the data will show a first order dependence with acid at very low acid concentrations only. Terms in the denominator in equation 6.16 become viable, as acid concentrations are increased, and the reaction will show saturation at these concentrations. This is what we observed in experiments.

Equation 6.17 also shows that the reaction will display first order kinetics with respect to chlorite and AETSA, irrespective of the strength of the acid.

\subsubsection{Consumption of AETSA}

AETSA has a weak absorption peak at $241 \mathrm{~nm}$, as illustrated in figure $6.8 \mathrm{a}$, but, fortunately, this peak is isolated. This peak can be used in conjunction with our knowledge of oxyhalogen chemistry, together with the data on induction periods, to evaluate the mechanistic details of the consumption of AETSA by chlorite. Figure $6.8 \mathrm{~b}$ shows the complex dependence of the consumption of AETSA on acid concentrations. 


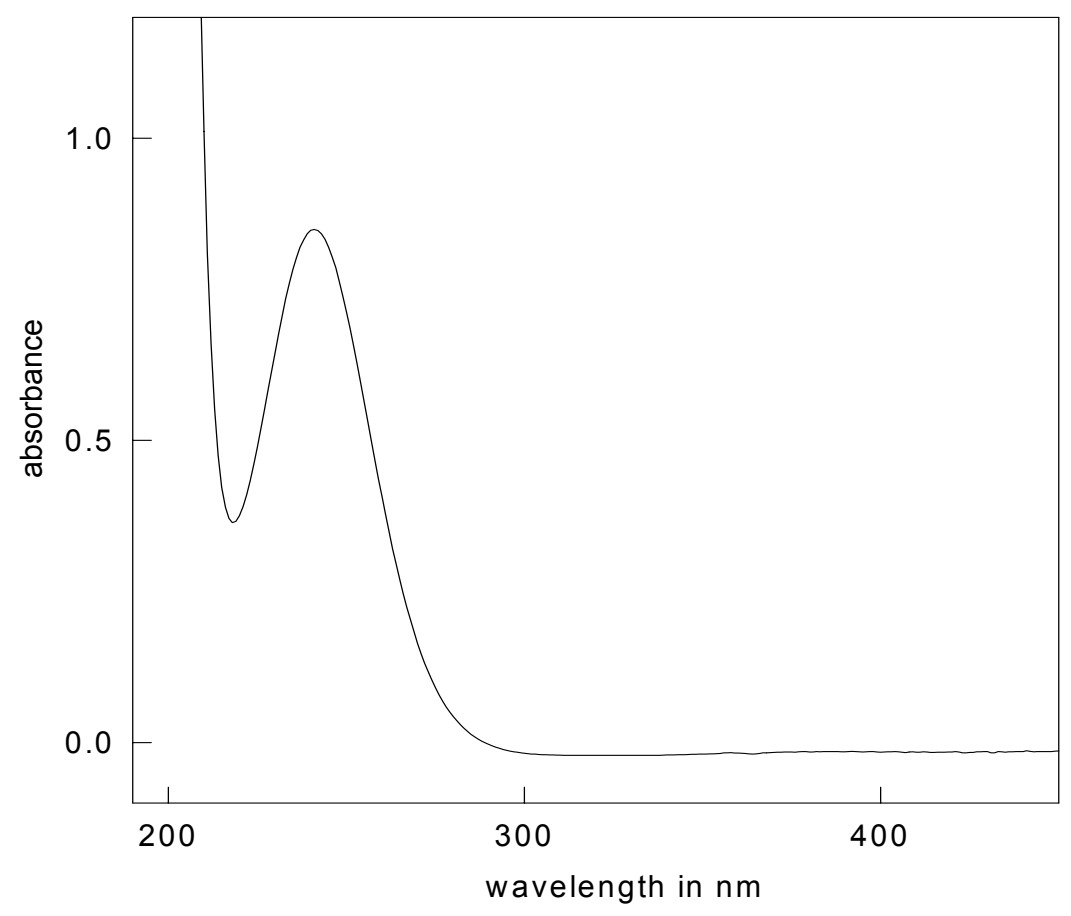

Figure 6.8a: Absorption spectrum of AETSA showing peak at $241 \mathrm{~nm}$. [AETSA] $=0.02 \mathrm{M}$, Extinction coefficient at $241 \mathrm{~nm}$ is $42.45 \mathrm{M}^{-1} \mathrm{~cm}^{-1}$

Although acid seems to catalyze the reaction, this effect quickly diminishes as acid concentrations are increased beyond $\mathrm{pH} 2.0$ (see figure 6.8c). Reactions 6.9 and 6.10 represent the initial steps in the oxidation of AETSA. The $\mathrm{HOCl}$ formed in reaction 6.9 and 6.10 reacts rapidly with any remaining $\mathrm{ClO}_{2}{ }^{-}$in solution to produce $\mathrm{ClO}_{2}$ [32]:

$$
2 \mathrm{ClO}_{2}^{-}+\mathrm{HOCl}+\mathrm{H}^{+} \rightarrow 2 \mathrm{ClO}_{2}+\mathrm{Cl}^{-}+\mathrm{H}_{2} \mathrm{O}
$$

If the reaction between $\mathrm{ClO}_{2}$ and AETSA is slow, then there will be an immediate accumulation of $\mathrm{ClO}_{2}$ (yellow) at the beginning of the reaction, and no induction period will be observed. Since an induction period was observed for this reaction, it would appear that the $\mathrm{ClO}_{2}$ formed in 6.18 is immediately wiped up by AETSA (and its 
derivatives that have not been oxidatively saturated). $\mathrm{HOCl}$ can also oxidize both AETSA and its derivatives.

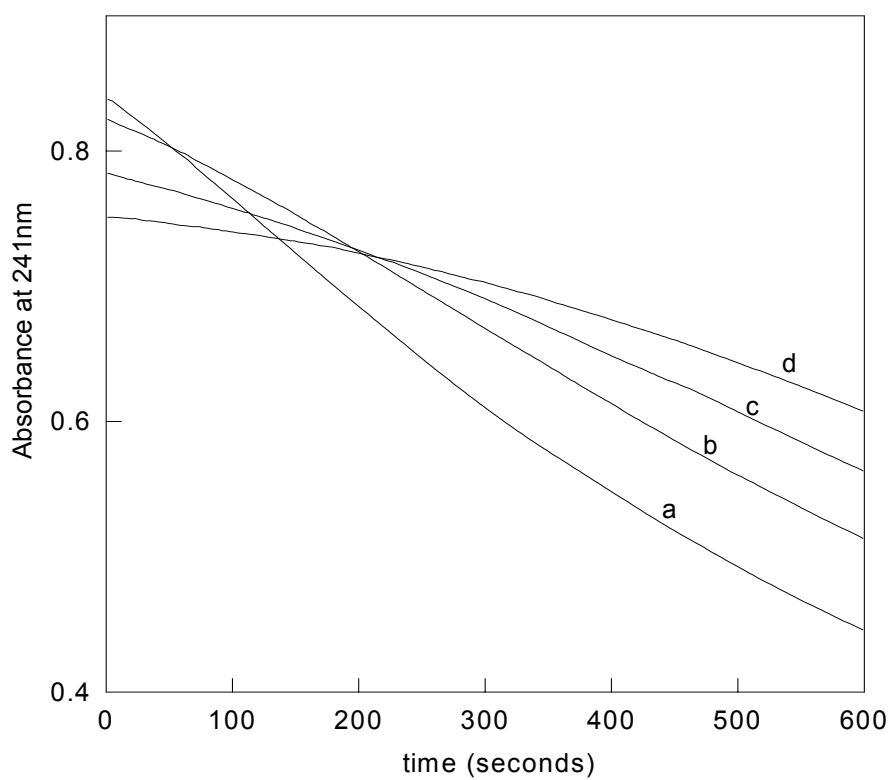

Figure 6.8b: Absorbance traces showing consumption of AETSA by chlorite at varying $\left[\mathrm{H}^{+}\right]$. $\left[\mathrm{ClO}_{2}{ }^{-}\right]=.005 \mathrm{M} ;[$ AETSA $]=0.005 \mathrm{M}, \mathrm{I}_{(\mathrm{NaCl})}=1.0 \mathrm{M}$ and $\left[\mathrm{H}^{+}\right]=(\mathrm{a}) 0.20 \mathrm{M} ;$ (b) $0.10 \mathrm{M} ;$ (c) $0.05 \mathrm{M}$; (d) $0.025 \mathrm{M}$.

$$
\begin{aligned}
& \mathrm{HOCl}+\mathrm{H}_{2} \mathrm{~N}-\mathrm{CH}_{2}-\mathrm{CH}_{2}-\mathrm{S}-\mathrm{SO}_{3} \mathrm{H} \rightarrow \mathrm{H}_{2} \mathrm{~N}-\mathrm{CH}_{2}-\mathrm{CH}_{2}-\mathrm{S}(\mathrm{O})-\mathrm{SO}_{3} \mathrm{H}+\mathrm{Cl}^{-}+\mathrm{H}^{+} \text {(fast) } \\
& \mathrm{HOCl}+\mathrm{H}_{2} \mathrm{~N}-\mathrm{CH}_{2}-\mathrm{CH}_{2}-\mathrm{S}(\mathrm{O})-\mathrm{SO}_{3} \mathrm{H} \rightarrow \mathrm{H}_{2} \mathrm{~N}-\mathrm{CH}_{2}-\mathrm{CH}_{2}-\mathrm{S}\left(\mathrm{O}_{2}\right)-\mathrm{SO}_{3} \mathrm{H}+\mathrm{Cl}^{-}+\mathrm{H}^{+}
\end{aligned}
$$

It is highly unlikely that the sulfone formed in reaction 6.20 is stable, and should thus be easily oxidized to sulfate and taurine.

$$
\mathrm{HOCl}+\mathrm{H}_{2} \mathrm{~N}-\mathrm{CH}_{2}-\mathrm{CH}_{2}-\mathrm{S}\left(\mathrm{O}_{2}\right)-\mathrm{SO}_{3} \mathrm{H}+\mathrm{H}_{2} \mathrm{O} \rightarrow \mathrm{H}_{2} \mathrm{~N}-\mathrm{CH}_{2}-\mathrm{CH}_{2}-\mathrm{SO}_{3} \mathrm{H}+\mathrm{SO}_{4}{ }^{2-}+\mathrm{Cl}^{-}+\mathrm{H}^{+}
$$




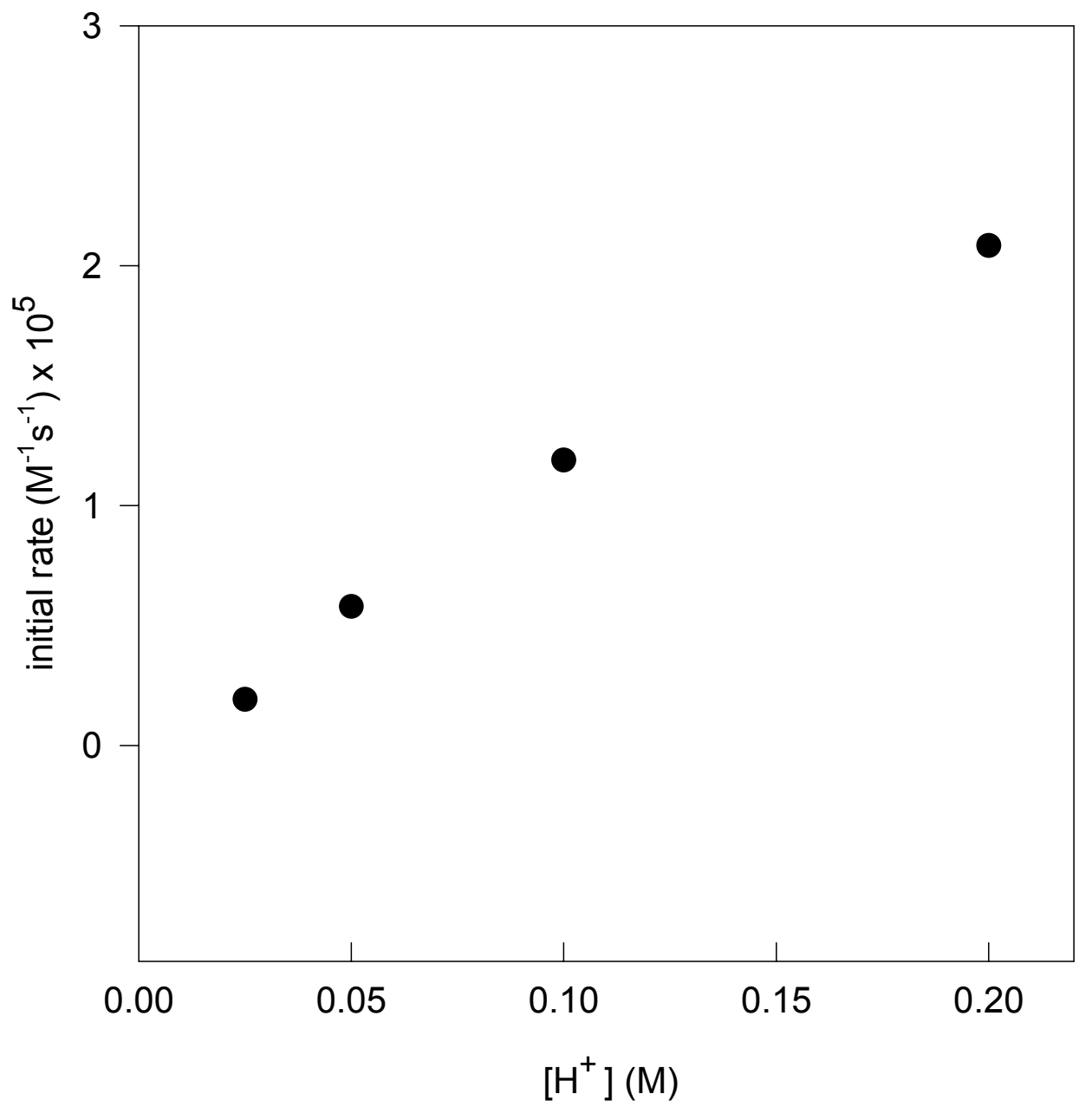

Figure 6.8c: Plot of initial rate versus $\left[\mathrm{H}^{+}\right]$showing a hint of saturation kinetics as acid concentrations are increased.

Though $\mathrm{ClO}_{2}{ }^{-}$can also perform the oxidations listed in reactions $6.19-6.21$, the bulk of these will be borne by $\mathrm{HOCl}$. This is due to the rapid and autocatalytic formation of $\mathrm{HOCl}$ from $\mathrm{ClO}_{2}^{-}$. These autocatalytic steps involve the well-known asymmetric intermediate $\mathrm{Cl}_{2} \mathrm{O}_{2}[33,34]$ :

$$
\begin{aligned}
& \mathrm{ClO}_{2}{ }^{-}+\mathrm{HOCl}+\mathrm{H}^{+} \rightarrow \mathrm{Cl}_{2} \mathrm{O}_{2}+\mathrm{H}_{2} \mathrm{O} \\
& \mathrm{Cl}_{2} \mathrm{O}_{2}+2 \mathrm{H}^{+}+\mathrm{Red} \rightarrow 2 \mathrm{HOCl}+\mathrm{Red}^{2+}
\end{aligned}
$$


Where 'Red' represents any 2-electron reductant in solution: AETSA, the sulfoxide and the sulfone. Combining reactions 6.22 and 6.23 shows the (quadratic) autocatalytic formation of $\mathrm{HOCl}$ :

$$
\mathrm{ClO}_{2}{ }^{-}+\mathrm{HOCl}+3 \mathrm{H}^{+}+\mathrm{Red} \rightarrow 2 \mathrm{HOCl}+\mathrm{H}_{2} \mathrm{O}+\mathrm{Red}^{2+}
$$

With such a rapid formation of $\mathrm{HOCl}$, all oxidations by chlorite will be insignificant. Since the rate of formation of $\mathrm{HOCl}$ is rate-determining, then reactions 6.9 and 6.10 will still be rate-determining. Induction period will end when reaction 6.18 produces more $\mathrm{ClO}_{2}$ than that which can be consumed by AETSA, the sulfoxide and the sulfone.

\subsubsection{Reaction of chlorine dioxide with AETSA}

Chlorine dioxide is an odd-electron species, and in most of its oxidations, the initial step involves the addition of an electron to produce chlorite:

$$
\mathrm{ClO}_{2}+\mathrm{e}^{-} \rightarrow \mathrm{ClO}_{2}^{-}
$$

The chlorite then carries out most of the oxidations. Reaction 6.25, however, will not be fast. Further reduction of $\mathrm{ClO}_{2}^{-}$will produce $\mathrm{HOCl}$, which will reproduce $\mathrm{ClO}_{2}$ according to reaction 6.18. This will not be very helpful with respect to the reduction of AETSA, and one would expect a very slow reaction that is controlled by the free-radical kinetics of reaction 6.25. Fortunately, $\mathrm{HOCl}$ reaction with AETSA is fast enough to compete favorably with 6.18 in producing $\mathrm{Cl}^{-}$(also produced in reactions $6.19-6.21$ ) 
which can directly attack $\mathrm{ClO}_{2}$ in acidic environments to produce more reactive species $\mathrm{ClO}_{2}{ }^{-}$and $\mathrm{HOCl}[35]$ :

$$
2 \mathrm{ClO}_{2}+\mathrm{Cl}^{-}+\mathrm{H}_{2} \mathrm{O} \rightarrow 2 \mathrm{ClO}_{2}^{-}+\mathrm{HOCl}+\mathrm{H}^{+}
$$

Reaction 6.26 (reverse of 6.18) is not a single step process, involves the initial formation of some powerful oxidizing intermediate species such as the asymmetric $\mathrm{Cl}_{2} \mathrm{O}_{2}$.

$$
\begin{aligned}
& \mathrm{Cl}_{2} \mathrm{O}_{2}^{-}+\mathrm{ClO}_{2} \rightleftharpoons \mathrm{ClO}_{2}^{-}+\mathrm{Cl}_{2} \mathrm{O}_{2} \\
& \mathrm{Cl}_{2} \mathrm{O}_{2}+\mathrm{H}_{2} \mathrm{O} \rightleftharpoons \mathrm{ClO}_{2}^{-}+\mathrm{HOCl}+\mathrm{H}^{+}
\end{aligned}
$$

The formation of these powerful oxidizing species would then initiate the oxidation, thereby by-passing the bottleneck established by reaction 6.25 . Since reaction 6.26 is the reverse of 6.18 , its direction is determined by $\mathrm{HOCl}$ concentrations. Reaction 6.18 should dominate in high $\mathrm{HOCl}$ concentrations.

Data in Figure 6.7 shows what appears to be a violation of the second law of thermodynamics by the delivery a non-monotonic decrease to the position of lowest free energy with respect to chlorine dioxide concentrations (see traces a and b). The observed 'cusp' in the decay of $\mathrm{ClO}_{2}$ can only be produced by a combination of two or more nonlinear kinetics pathways. These could be in the form of autocatalysis or autoinhibition. Quadratic autocatalysis is displayed in the production of $\mathrm{HOCl}$, and this fuels its rapid increase in which not all $\mathrm{HOCl}, \mathrm{Cl}^{-}$, and $\mathrm{ClO}_{2}{ }^{-}$has been consumed by the time the reducing substrate, AETSA has been completely consumed. From the above 
reaction network, it is not difficult to explain the observed increase in $\mathrm{ClO}_{2}$ at the end of the reaction in Figure 6.7. The system can proceed according to 6.26 until all the AETSA has been consumed, leaving behind $\mathrm{ClO}_{2}{ }^{-}, \mathrm{Cl}^{-}$, and some $\mathrm{HOCl}$. These will recombine in the absence of reductant to re-form $\mathrm{ClO}_{2}$. This reaction (that re-forms $\mathrm{ClO}_{2}$ ) is necessary for stoichiometric consistency.

\subsubsection{Formation of $\mathrm{ClO}_{2}$}

Most oxyhalogen reactions are catalyzed by acid. For this particular system, the opposite is observed, i.e. a retardation of $\mathrm{ClO}_{2}$ formation with acid (see Figure 6.4). $\mathrm{ClO}_{2}$ is formed by the oxidation of chlorite. This oxidation can be carried out by either $\mathrm{HOCl}$ or $\mathrm{Cl}_{2}$ :

$$
2 \mathrm{Cl}(\mathrm{III})+\mathrm{Cl}(\mathrm{I}) \rightarrow 2 \mathrm{Cl}(\mathrm{IV})+\mathrm{Cl}(-\mathrm{I})
$$

and

$$
\mathrm{Cl}(\mathrm{III})+\mathrm{Cl}(0) \rightarrow \mathrm{Cl}(\mathrm{IV})+\mathrm{Cl}(-\mathrm{I})
$$

It is reasonable to assume that reaction 6.18 (in the form of 6.28 , above), would be the dominant route for the production of $\mathrm{ClO}_{2}$. Reaction 6.18 is a composite reaction that involves the initial reaction of $\mathrm{ClO}_{2}^{-}$with $\mathrm{HOCl}$ (as in reaction 6.22). The protonated form of $\mathrm{ClO}_{2}^{-}$; chlorous acid, $\mathrm{HClO}_{2}$, is a poorer nucleophile than the unprotonated form, $\left(\mathrm{ClO}_{2}^{-}\right)(\mathrm{HOCl}$ is the electrophile $)$, and hence in high acid, contributions from reaction 6.18 become minimal in favor of the slower reaction of $\mathrm{HOCl}$ with $\mathrm{HClO}_{2}$ : 


$$
2 \mathrm{HClO}_{2}+\mathrm{HOCl} \rightarrow 2 \mathrm{ClO}_{2}+\mathrm{Cl}^{-}+\mathrm{H}^{+}+\mathrm{H}_{2} \mathrm{O}
$$

or simply there will be less free $\mathrm{ClO}_{2}{ }^{-}$available to effect reaction 6.18 .

\subsubsection{Quantitative formation of chlorine dioxide}

By incubating reaction solutions for extended periods of time ( 7 days or more), one notices that there was still a slower rate of formation of chlorine dioxide that continued way after all the AETSA had been consumed. Since we utilized highly acidic conditions, we expect disproportionation of chlorite to chlorine dioxide over the other pathways that give chlorate (in basic environments). Both stoichiometries 6.1 and 6.2 give $\mathrm{Cl}^{-}$ions as products. In the presence of acid, the following slow reaction will occur [36]:

$$
\mathrm{ClO}_{2}^{-}+\mathrm{Cl}^{-}+2 \mathrm{H}^{+} \rightleftharpoons 2 \mathrm{HOCl}
$$

The production of $\mathrm{HOCl}$ will immediately trigger reaction 6.18 , forming chlorine dioxide. Since reaction 6.30 is slow when compared to the time scale of the oxidation of AETSA, it will not interfere with the kinetics of the reaction under study. It will, however, be responsible for the continued increase in $\mathrm{ClO}_{2}$ concentrations way after the oxidation of AETSA is complete. By eliminating $\mathrm{HOCl}$, one can add 2(6.18) +6.30 to obtain a well-known reaction for the decomposition of $\mathrm{ClO}_{2}{ }^{-}$in acidic an medium [32]

$$
5 \mathrm{ClO}_{2}^{-}+4 \mathrm{H}^{+} \rightarrow 4 \mathrm{ClO}_{2}+2 \mathrm{H}_{2} \mathrm{O}+\mathrm{Cl}^{-}
$$


Ultimately, the final chlorine dioxide formed depends on the excess chlorite concentrations according to reaction stoichiometry 6.31. In mildly acidic environments, it can take several days for stoichiometry 6.31 to be established.

\subsubsection{Computer Simulations}

The full mechanism which combines the chlorite-AETSA reactions and the chlorine dioxide-aetsa reactions was compiled into 21 reactions as shown in Table 6.1. The reaction mechanism is made up of 12 oxychlorine-sulfur reactions in which the chlorine center is reduced, 4 pure oxyhalogen reactions, 3 sulfur-sulfur disproportionation reactions, and 2 acid-base equilibria. The stochastic algorithm contained in the Chemical Kinetics Simulator (CKS) software package developed by IBM's Almaden Research Center was used to model this reaction network.

Reactions M1 and M21 are rapid protolytic reactions whose kinetics parameters were inconsequential on the simulations for as long as they were fast (and not ratedetermining). The ratios of the forward and reverse reactions were chosen such that they maintained the standard $\mathrm{p} K_{\mathrm{a}} \mathrm{s}$ of the respective weak acids. The parameters for the oxychlorine reactions, M11, M12, M17 and M18 were derived from a combination of literature values and initial guess estimates [37-40]. The most important parameter in determining the clock-nature of the overall reaction turned out to be the reverse rate constant for M12, $k_{\mathrm{m} 12}$. Several authors have worked on the kinetics of reaction M11 [36]. Reaction M11 was not treated as termolecular reaction computationally. The acid concentration was high enough for us to assume buffering, and so the rate constant $k_{11}=$ $1.01 \times 10^{6} \mathrm{M}^{-2} \mathrm{~s}^{-1}$ reported in the literature was replaced by a pseudo-rate constant, $k_{11}$ ' = 
$k_{11}\left[\mathrm{H}^{+}\right]_{0}$ where $\left[\mathrm{H}^{+}\right]_{0}$ was assumed constant throughout the reaction lifetime.

TABLE 6.1 FULL MECHANISMS FOR THE CHLORITE-AETSA REACTION

Rate constants used are shown after the semi-colon. For a reversible reaction, the reverse rate constant is shown after a comma. Except where water is involved, the order of the reaction is derived from the molecularity.

\begin{tabular}{|c|c|c|c|}
\hline & REACTANTS & & PRODUCTS \\
\hline M1. & $\mathrm{ClO}_{2}^{-}+\mathrm{H}^{+}$ & $\rightleftharpoons$ & $\mathrm{HClO}_{2} ; 1 \times 10^{9}, 1.91 \times 10^{7}$ \\
\hline M2. & $\mathrm{ClO}_{2}^{-}+\mathrm{RS}-\mathrm{SO}_{3} \mathrm{H}$ & $\rightarrow$ & $\mathrm{RS}(\mathrm{O}) \mathrm{SO}_{3} \mathrm{H}+{ }^{-} \mathrm{OCl} ; 5.80$ \\
\hline M3. & $\mathrm{HClO}_{2}+\mathrm{RS}-\mathrm{SO}_{3} \mathrm{H}$ & $\rightarrow$ & $\mathrm{RS}(\mathrm{O}) \mathrm{SO}_{3} \mathrm{H}+\mathrm{HOCl} ; 3.20$ \\
\hline M4. & $\mathrm{ClO}_{2}^{-}+\mathrm{RS}(\mathrm{O}) \mathrm{SO}_{3} \mathrm{H}$ & $\rightarrow$ & $\mathrm{RS}\left(\mathrm{O}_{2}\right) \mathrm{SO}_{3} \mathrm{H}+{ }^{-} \mathrm{OCl} ; 45$ \\
\hline M5. & $\mathrm{ClO}_{2}^{-}+\mathrm{RS}\left(\mathrm{O}_{2}\right) \mathrm{SO}_{3} \mathrm{H}+\mathrm{H}_{2} \mathrm{O}$ & $\rightarrow$ & $\mathrm{RSO}_{3} \mathrm{H}+\mathrm{SO}_{4}^{2-}+\mathrm{H}^{+}+\mathrm{HOCl} ; 5 \times 10^{3}$ \\
\hline M6. & $\mathrm{HOCl}+\mathrm{RS}-\mathrm{SO}_{3} \mathrm{H}$ & $\rightarrow$ & $\mathrm{RS}(\mathrm{O}) \mathrm{SO}_{3} \mathrm{H}+\mathrm{Cl}^{-}+\mathrm{H}^{+} ; 43.8$ \\
\hline M7. & $\mathrm{HOCl}+\mathrm{RS}(\mathrm{O}) \mathrm{SO}_{3} \mathrm{H}$ & $\rightarrow$ & $\mathrm{RS}\left(\mathrm{O}_{2}\right) \mathrm{SO}_{3} \mathrm{H}+\mathrm{Cl}^{-}+\mathrm{H}^{+} ; 5 \times 10^{2}$ \\
\hline M8. & $\mathrm{HOCl}+\mathrm{RS}\left(\mathrm{O}_{2}\right) \mathrm{SO}_{3} \mathrm{H}+\mathrm{H}_{2} \mathrm{O}$ & $\rightarrow$ & $\mathrm{RSO}_{3} \mathrm{H}+\mathrm{SO}_{4}^{2-}+\mathrm{Cl}^{-}+3 \mathrm{H}^{+} ; 1 \times 10^{3}$ \\
\hline M9. & $\mathrm{HOCl}+\mathrm{RSO}_{3} \mathrm{H}$ & $\rightarrow$ & $\mathrm{ClHNCH}_{2} \mathrm{CH}_{2} \mathrm{SO}_{3} \mathrm{H}+\mathrm{H}_{2} \mathrm{O} ; 5 \times 10^{6}$ \\
\hline M10. & $2 \mathrm{ClHNCH}_{2} \mathrm{CH}_{2} \mathrm{SO}_{3} \mathrm{H}$ & $\rightleftharpoons$ & $\mathrm{RSO}_{3} \mathrm{H}+\mathrm{Cl}_{2} \mathrm{NCH}_{2} \mathrm{CH}_{2} \mathrm{SO}_{3} \mathrm{H} ; 5 \times 10^{7}, 1 \times 10^{6}$ \\
\hline M11. & $\mathrm{ClO}_{2}^{-}+\mathrm{HOCl}+\mathrm{H}^{+}$ & $\rightleftharpoons$ & $\mathrm{Cl}_{2} \mathrm{O}_{2}+\mathrm{H}_{2} \mathrm{O} ; 1.01 \times 10^{6}, 1 \times 10^{-1}$ \\
\hline M12. & $\mathrm{Cl}_{2} \mathrm{O}_{2}+\mathrm{ClO}_{2}^{-}$ & $\rightleftharpoons$ & $2 \mathrm{ClO}_{2}+\mathrm{Cl}^{-} ; 1.5 \times 10^{3}, 5.5 \times 10^{6}$ \\
\hline M13. & $\mathrm{Cl}_{2} \mathrm{O}_{2}+\mathrm{RS}-\mathrm{SO}_{3} \mathrm{H}+\mathrm{H}_{2} \mathrm{O}$ & $\rightarrow$ & $\mathrm{RS}(\mathrm{O}) \mathrm{SO}_{3} \mathrm{H}+2 \mathrm{HOCl} ; 15.5$ \\
\hline M14. & $\mathrm{Cl}_{2} \mathrm{O}_{2}+\mathrm{RS}(\mathrm{O}) \mathrm{SO}_{3} \mathrm{H}+\mathrm{H}_{2} \mathrm{O}$ & $\rightarrow$ & $\mathrm{RS}\left(\mathrm{O}_{2}\right) \mathrm{SO}_{3} \mathrm{H}+2 \mathrm{HOCl} ; 17.5$ \\
\hline M15. & $\mathrm{Cl}_{2} \mathrm{O}_{2}+\mathrm{RS}\left(\mathrm{O}_{2}\right) \mathrm{SO}_{3} \mathrm{H}+2 \mathrm{H}_{2} \mathrm{O}$ & $\rightarrow$ & $\mathrm{RSO}_{3} \mathrm{H}+\mathrm{SO}_{4}{ }^{2-}+2 \mathrm{HOCl}+2 \mathrm{H}^{+} ; 5 \times 10^{3}$ \\
\hline M16. & $2 \mathrm{ClO}_{2}+\mathrm{RS}-\mathrm{SO}_{3} \mathrm{H}+\mathrm{H}_{2} \mathrm{O}$ & $\rightarrow$ & $2 \mathrm{ClO}_{2}^{-}+\mathrm{RS}(\mathrm{O}) \mathrm{SO}_{3} \mathrm{H}+2 \mathrm{H}^{+} ; 5 \times 10^{6}$ \\
\hline
\end{tabular}




\begin{tabular}{|c|c|c|c|}
\hline M17. & $\mathrm{ClO}_{2}+\mathrm{Cl}^{-}$ & $\rightleftharpoons$ & $\mathrm{Cl}_{2} \mathrm{O}_{2}^{-} ; 4 \times 10^{6}, 5 \times 10^{5}$ \\
\hline M18. & $\mathrm{Cl}_{2} \mathrm{O}_{2}^{-}+\mathrm{ClO}_{2}$ & $\rightleftharpoons$ & $\mathrm{ClO}_{2}^{-}+\mathrm{Cl}_{2} \mathrm{O}_{2} ; 5 \times 10^{3}, 5 \times 10^{2}$ \\
\hline M19. & $2 \mathrm{RS}(\mathrm{O}) \mathrm{SO}_{3} \mathrm{H}$ & $\rightleftharpoons$ & $\mathrm{RS}-\mathrm{SO}_{3} \mathrm{H}+\mathrm{RS}\left(\mathrm{O}_{2}\right) \mathrm{SO}_{3} \mathrm{H} ; 1 \times 10^{6}, 3 \times 10^{7}$ \\
\hline M20. & $\mathrm{RS}(\mathrm{O}) \mathrm{SO}_{3} \mathrm{H}+\mathrm{RS}\left(\mathrm{O}_{2}\right) \mathrm{SO}_{3} \mathrm{H}+\mathrm{H}_{2} \mathrm{O}$ & $\rightleftharpoons$ & $\mathrm{RS}-\mathrm{SO}_{3} \mathrm{H}+\mathrm{RSO}_{3} \mathrm{H}+\mathrm{SO}_{4}{ }^{2-}+2 \mathrm{H}^{+} ; 50,0.01$ \\
\hline M21. & ${ }^{-} \mathrm{OCl}+\mathrm{H}^{+}$ & $\rightleftharpoons$ & $\mathrm{HOCl} ; 1 \times 10^{9}, 5 \times 10^{5}$ \\
\hline
\end{tabular}

Legend:

$\mathrm{R}=\mathrm{H}_{2} \mathrm{NCH}_{2} \mathrm{CH}_{2}-$

$\mathrm{RSO}_{3} \mathrm{H}=$ Taurine

The oxychlorine-sulfur reactions were derived from a pure permutation of three oxidants $\left(\mathrm{ClO}_{2}^{-}, \mathrm{HOCl}\right.$ and $\left.\mathrm{Cl}_{2} \mathrm{O}_{2}\right)$ with four reductants (AETSA, the sulfoxide, the sulfone and taurine). Experimental data was then helpful in determining the initial guess value for each process. For example, NMR data showed the evolution of a stable sulfoxide, and thus the further oxidation of the sulfoxide to the sulfone was assumed to be relatively slow compared to the further oxidation of the sulfone. Reaction M10, was in most cases inconsequential in the oxidation of AETSA, but was included in very highly acidic conditions due to the formation of the protonated taurine molecule that tended to pull the equilibrium of M10 more to the right. Reactions M19 and M20 are disproportionation reactions whose sole role is to ensure stoichiometric consistency after complete consumption of either the oxidant or AETSA. Thus, it was not at all important what rate constants were given for these reactions, as long as they were fast enough to establish equilibrium within the same time frame of the chlorite-AETSA reaction. High values of kinetic parameters made the simulations stiff and slow, while too low values, introduced fluctuations in AETSA and oxychlorine species which we had not 
experimentally observed. All reactions involving oxidation by the intermediate, $\mathrm{Cl}_{2} \mathrm{O}_{2}$, were deliberately slowed so that all the observed autocatalysis could be derived from the production of $\mathrm{HOCl}$ (reactions M12 to M15). Autocatalysis was then clearly evident if $\mathrm{HOCl}$ oxidations are faster than those by either $\mathrm{ClO}_{2}{ }^{-}$or $\mathrm{Cl}_{2} \mathrm{O}_{2}$.

As expected, the most important kinetic parameter in this whole reaction mechanism was $k_{2}$ (forward rate constant for reaction M2). The value chosen for our simulation; $5.8 \mathrm{M}^{-2} \mathrm{~s}^{-1}$, was chosen by estimating the initial rate of depletion of AETSA using the data shown in figure $6.8 \mathrm{a}$, and then working around that estimate for the best fit. The selected value of $k_{3}$, after establishment of $k_{2}$, was only useful in determining the degree of acid retardation in the formation of chlorine dioxide after the induction period. Otherwise one could completely shut down M3 and rely on the equilibrium in reaction M1 to handle the acid retardation.

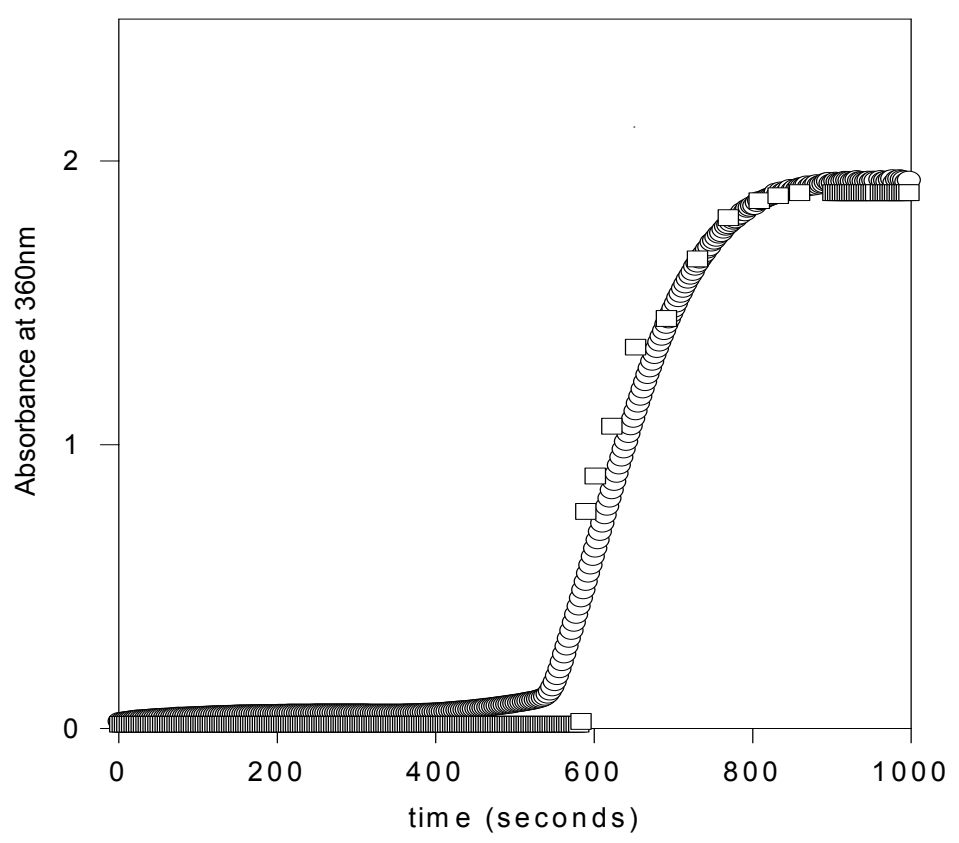

Figure 6.9: Results of modeling reaction mechanism shown in Table 1 for the conditions $\left[\mathrm{ClO}_{2}{ }^{-}\right.$ ]$_{0}=0.004 \mathrm{M} ;[\text { AETSA }]_{0}=0.001 \mathrm{M} ;\left[\mathrm{H}^{+}\right]_{0}=0.08 \mathrm{M}$. Data points generated by the model are shown as the square symbols. 
There was reasonable agreement between our model (Table 6.1) and our experimental data. The chlorine dioxide-AETSA reaction could also be modeled by the same mechanism by assuming an initial chlorite concentration of zero. Figure 6.9 shows our fit to a typical trace under conditions of excess oxidant. Though simulations of the chlorine dioxide-AETSA reaction gave good agreement for the consumption of $\mathrm{ClO}_{2}$, the model, however, gave a zero order-type of trace for the re-formation of $\mathrm{ClO}_{2}$ (as opposed to the 'cusp' observed in Figure 6.7).

\subsection{CONCLUSION}

Studies on the oxidation of the Bunte salt, AETSA, by chlorite have shown that the reaction is characterized by a long quiescent induction period followed by a rapid, autocatalytic production of chlorine dioxide. The results obtained revealed that AETSA was a good precursor for taurine in acidic oxidizing environments, due to cleavage of the S-S bond after a single S-oxygenation step on the inner sulfur atom. The sulfoxide was quite stable as shown by NMR experiments. There was no experimental evidence for existence of the sulfone-sulfonic acid, and sulfate production was almost quantitative for the oxidation of only one of the sulfur atoms. Further reaction of the taurine occurred only on the nitrogen atom with no cleavage of the $\mathrm{C}-\mathrm{S}$ bond. Thus, some of the physiological effects attributed to AETSA might actually be derived from taurine itself or its derivatives. This proves that the $\mathrm{S}-\mathrm{S}$ bond is very important in physiological mechanisms and elucidation of its oxidation mechanism is the first step towards characterizing its physiological effects. 


\section{References}

1. C. R. Chinake, C. Mundoma, R. Olojo, T. R. Chigwada and R. H. Simoyi, "Oxyhalogen-sulfur chemistry: Kinetics and mechanism of the oxidation of a Bunte salt 2-aminoethanethiolsulfuric acid by chlorite," Phys. Chem. Chem. Phys. 3, 4957-4964 (2001).

2. E. Mambo and R. H. Simoyi, "Kinetics and mechanism of the complex oxidation of aminoiminomethanesulfinic acid by iodate in acidic medium," J. Phys. Chem. 97, 13662-13667 (1993).

3. R. F. Grimble, "Sulfur amino acids and the metabolic response to cytokines," in Taurine in health and disease vol. 359, edited by R. J. Huxtable and D. V. Michalk, (Plenum Press, New York, 1994) pp. 41-49.

4. R. J. Huxtable, A. Barbeau, and W. G. Weston, "Taurine", in International Symposium on Taurine, (Raven Press, New York, 1976).

5. R. J. Huxtable and H. Pasantes-Morales, Taurine in Nutrition and Neurology, (Plenum Press, New York, 1982).

6. R. J. Huxtable, F. Franconi, and A. Giotti, The Biology of Taurine: Methods and Mechanisms, (Plenum Press, New York, 1987).

7. A. W. R. Tyrrell, "Synthesis of Bunte salts from 1,2-benzisothiazol-3-ones and vice versa," Tetrahedron Lett. 26, 1753-1756 (1985).

8. R. J. Huxtable, "Physiological actions of taurine," Physiol. Rev. 72, 101-163 (1992).

9. M. Shimada, R. Shimono, M. Watanabe, T. Imahayashi, H. S. Ozaki, T. Kihara, K. Yamaguchi, and S. Niizeki, "Distribution of ${ }^{35}$ S-Taurine in rat neonates and adults. A whole-body autoradiographic study," Histochemistry 80, 225-230 (1984).

10. B. Qi, T. Yamagami, Y. Naruse, S. Sokejima, and S. Kagamimori, "Effects of taurine on depletion of erythrocyte membrane Na-K ATPase activity due to ozone exposure or cholesterol enrichment," J. Nutr. Sci. Vitaminol. 41, 627-634 (1995).

11. C. Wu, C. Miyagawa, D. O. Kennedy, Y. Yano, S. Otani, and I. Matsui-Yuasa, "Involvement of polyamines in the protection of taurine against the cytotoxicity of hydrazine or carbon tetrachloride in isolated rat hepatocytes," Chem. Bio. Interact. 103, 213-224 (1997).

12. J. A. Timbrell and C. J. Waterfield, "Changes in taurine as an indicator of hepatic dysfunction and biochemical perturbations: studies in vivo and in vitro," Adv. Exp. Med. Biol. 403, 125-134 (1996). 
13. C. J. Waterfield, F. Carvalho, and J. A. Timbrell, "Effect of treatment with bagonists on tissue and urinary taurine levels in rats: Mechanism and implications for protection," Adv. Exp. Med. Biol. 403, 233-245 (1996).

14. C. J. Waterfield, J. A. Turton, M. D. Scales, and J. A. Timbrell, "The correlation between urinary and liver taurine levels and between pre-dose urinary taurine and liver damage," Toxicology 77, 1-5 (1993).

15. C. J. Waterfield, J. A. Turton, M. D. Scales and J. A. Timbrell, "Reduction of liver taurine in rats by b-alanine treatment increases carbon tetrachloride toxicity," Toxicology 77, 7-20 (1993).

16. C. J. Waterfield, M. Mesouita, P. Parnham, and J. A. Timbrell, "Taurine protects against the cytotoxicity of hydrazine, 1,4-naphthoquinone and carbon tetrachloride in isolated rat hepatocytes," Biochem. Pharmacol. 46, 589-595 (1993).

17. T. Nakashima, T. Taniko, and K. Kuriyama, "Therapeutic effect of taurine administration on carbon tetrachloride-induced hepatic injury," Jpn. J. Pharmacol. 32, 583-589 (1982).

18. N. M. Van Gelder, "Brain taurine content as a function of cerebral metabolic rate: osmotic regulation of glucose derived water production," Neurochem. Res. 14, 495497 (1989).

19. S. Mizushima, Y. Nara, M. Sawamura, and Y. Yamori, "Effects of oral taurine supplementation on lipids and sympathetic nerve tone," Adv. Exp. Med. Biol. 403, 615-622 (1996).

20. H. Pasantes-Morales, J. Moran, and A. Schousboe, "Volume-sensitive release of taurine from cultured astrocytes: properties and mechanism," Glia 3, 427-432 (1990).

21. A. Schousboe, J. Moran, and H. Pasantes-Morales, "Potassium-stimulated release of taurine from cultured cerebella granule neurons is associated with cell swelling," J. Neurosci. Res. 27, 71-77 (1990).

22. H. Pasantes-Morales and R. Martin del Rio, "Taurine and mechanisms of cell volume regulation," Prog. Clin. Biol. Res. 351, 317-328 (1990).

23. H. Pasantes-Morales, J. Moran, and A. Schousboe, "Taurine release associated to cell swelling in the nervous system," Prog. Clin. Biol. Res. 351, 369-376 (1990).

24. H. Satoh and N. Sperelakis, "Effects of taurine on calcium currents in young embryonic chick cardiomyocytes," Eur. J. Pharmacol. 231, 443-449 (1993).

25. H. Satoh and N. Sperelakis, "Review of some actions of taurine on ion channels of cardiac muscle cells and others," Gen. Pharmacol. 30, 451-463 (1998). 
26. C. Mundoma and R. H. Simoyi, "Oxyhalogen-sulfur chemistry: Oxidation of 2aminoethanethiosulfuric acid by iodate in acidic medium," J. Chem. Soc., Faraday Trans. 93, 1543-1550 (1997).

27. J. Darkwa, C. Mundoma, and R. H. Simoyi, "Nonlinear dynamics in chemistry derived from sulfur chemistry. Part 21. Oxyhalogen-sulfur chemistry: Non-linear oxidation of 2-aminoethanethiolsulfuric acid (AETSA) by bromate in acidic medium," J. Chem. Soc. Faraday Trans. 92, 4407-4413 (1996).

28. Y. Y. Lin, C. E. Wright, M. Zagorski, and K. Nakanishi, " ${ }^{13}$ C-NMR study of taurine and chlorotaurine in human cells," Biochim. Biophys. Acta 969, 242-248 (1988).

29. C. R. Chinake and R. H. Simoyi, "Oxyhalogen-sulfur chemistry: Oxidation of taurine by chlorite in acidic medium," J. Phys. Chem. B 101, 1207-1214 (1997).

30. G. Rabai and M. T. Beck, "Oxidation of thiourea by iodate: A new type of oligooscillatory reaction," J. Chem. Soc. Dalton 8, 1669-1672 (1985).

31. P. W. Atkins and J. A. Beran, General chemistry, (Scientific American, New York, 1992).

32. Z. Jia, D. W. Margerum and J. S. Francisco, "General-acid-catalyzed reactions of hypochlorous acid and acetyl hypochlorite with chlorite ion," Inorg. Chem. 39, 2614-2620 (2000).

33. H. Taube and H. Dodgen, "Applications of radioactive chlorine to the study of the mechanisms of reactions involving changes in the oxidation state of chlorine," $\mathrm{J}$. Am. Chem. Soc. 71, 3330-3336 (1949).

34. H. Dodgen and H. Taube, "Exchange of chlorine dioxide with chlorite ion and with chlorine in other oxidation states," J. Am. Chem. Soc. 71, 2501-2504 (1949).

35. G. Rabai and M. Orban, General model for the chlorite ion based chemical oscillators. Journal of Physical Chemistry 97, 5935-5939 (1993).

36. G. Peintler, I. Nagypal and I. R. Epstein, "Systematic design of chemical oscillators. 60. Kinetics and mechanism of the reaction between chlorite ion and hypochlorous acid," J. Phys. Chem. 94, 2954-2958 (1990).

37. I. Lengyel, L. Gyorgyi and I. R. Epstein, "Analysis of a model of chlorite-based chaotic chemical oscillators," J. Phys. Chem. 99, 12804-12808 (1995).

38. J. N. Figlar and D. M. Stanbury, "Kinetics and a revised mechanism for the autocatalytic Oxidation of SCN by $\mathrm{ClO}_{2}$," J. Phys. Chem. A 103, 5732-5741 (1999).

39. J. N. Figlar and D. M. Stanbury, Kinetics and mechanism of the aqueous reaction of chlorine dioxide with thiocyanate in Book of Abstracts, $217^{\text {th }}$ ACS National Meeting, (1999). 
40. F. Emmenegger and G. Gordon, "The rapid interaction between sodium chlorite and dissolved chlorine," Inorg. Chem. 6, 633-635 (1967). 


\section{CHAPTER 7}

\section{Resonance Induced Chemical Waves}

\subsection{INTRODUCTION}

Chemical waves, which are examples of dissipative structures, often occur in nonlinear systems that are not at thermodynamic equilibrium [1]. They are comprised of concentration gradients that propagate in an excitable medium. Chemical waves were first characterized by Zaikin and Zhabotinsky in 1970 [2] and have been subject to extensive studies over the past three decades [3,4]. They arise from the coupling of autocatalytic reactions with diffusion and can be mathematically described by reactiondiffusion equations,

$$
\frac{\partial u}{\partial t}=\mathrm{D} \nabla^{2} u+\mathrm{F}[u]
$$

where D represents the diffusion coefficients of the various species present in the system, $u$ is a vector of the species concentrations or state variables, $\nabla^{2}$ is the Laplacian operator, and $\mathrm{F}[u]$ are the nonlinear functions of the state variables. Spatiotemporal patterns have been investigated on oxyhalogen systems involving iodate [5,6] and chlorite [7] reactions, although the venerable Belousov-Zhabotinsky (BZ) [8] reaction has been studied in greater depth [9]. Pattern formation has not been limited to chemical systems $[2,3,10]$ but has also been investigated in biochemical systems, such as the glycolytic system using a yeast extract [9,11], and biological systems [12-16], such as the cardiac muscle [17-19]. Pattern formation also occurs during the development of embryos [20,21], during peristaltic motion [22], and in nerve axons [23]. Conditions that must be

met for the onset of spatiotemporal patterns are given by the following [24]: (1) The 
system must be maintained in a nonequilibrium state in which reactants are continuously supplied and products removed. (2) To ensure that the appropriate nonlinear dynamical elements are present, some feedback process, such as autocatalysis or autoinhibition, must occur in the system.

The observance of spatial structures has long been recognized as an essential phenomenon in biological systems, which are open systems and thus need not follow the thermodynamic principle of increasing disorder. One of the most fascinating examples of spatiotemporal pattern formation is morphogenesis. In pioneering work, Turing [25] proposed that coupling between reaction and diffusion processes in nonlinear systems could give rise to such structures.

The pacemaker source for the genesis of propagating waves has attracted the attention of researchers for many years [26-29]. Ventricular tachycardia [16,30], for example, has been studied in the context of spurious wave initiations. Complex, unsynchronized and potentially dangerous rhythms in the cardiac system may arise from the abrupt starting and stopping of pacemakers $[31,32]$. Such irregularities can lead to ventricular fibrillation, a major cause of sudden cardiac death (SCD) $[13,33]$. The origin of these pacemaker (source) regions [32,34,35] still remains of interest, although questions such as how target patterns propagate from source regions have been successfully answered and the properties of chemical waves have been characterized $[1,4,9]$. Local inhomogeneities, usually due to dust particles [36], intrinsic and irregular thermal variations [27], and instabilities in wave propagation have been discussed as the source of pacemakers in literature [26,35]. 
There are many ways of initiating chemical waves in unstirred oscillatory or quiescent excitable reaction systems. Wave initiation can be achieved by locally perturbing the system [27], for example, by the local addition or depletion of chemical species [28]. Perturbations are typically carried out using a light source [37,38], via local heating [39], by mechanical stimulation in elastic BZ media [40], or electrochemically by locally depleting bromide ion using a silver electrode $[28,29,41]$. These methods rely on a local perturbation of the medium excitability by means of an accessible control variable $[27,42,43]$ beyond a critical threshold, and normally involves locally switching the system from an excitable steady state to an oscillatory state [32]. For example, removal of bromide ions from an excitable BZ medium at a silver electrode results in the initiation of waves as the system becomes locally oscillatory near the electrode $[28,29]$. Waves in the excitable photosensitive $\mathrm{BZ}$ reaction have been initiated via perturbations in light intensity (decreasing light intensity), which locally switches the system to the oscillatory state.

Recently, a new mechanism for the initiation of pacemakers in an excitable media was reported [32]. Rather than the traditional suprathreshold perturbations locally giving rise to an oscillatory state, waves where initiated in an excitable system via localized, small amplitude variations in light intensity (without crossing the bifurcation point of the autonomous system). Initiation of waves in the initially quiescent medium was possible when the frequency of the sinusoidal perturbation was suitably tuned to that of the autonomous system. The region in phase space where wave initiation was possible depended on the parameter values of the perturbation, namely forcing frequency and forcing amplitude, and on the inherent properties of the autonomous system. In this 
chapter we discuss further numerical investigations and experimental observations of this phenomenon.

\subsubsection{The Belousov-Zhabotinsky (BZ) Reaction}

Chemical waves in the excitable BZ reaction have been extensively studied as a chemical model for waves in excitable biological media, such as heart tissue, brain tissues, and neuronal tissue $[2,35,40,44,45]$. The $\mathrm{BZ}$ reaction displays many of the characteristic qualities of dissipative structures found in living systems [24].

The BZ reaction was discovered by B. P. Belousov, a Russian chemist, while he was investigating the oxidation of citric acid by potassium bromate under acidic conditions. He observed that the color of the homogeneous solution alternated between pale yellow and colorless at regular time intervals. Attempts at publishing his results in a number of journals where unsuccessful, as contemporary chemists believed his observations violated the laws of thermodynamics. A few years later, A. M. Zhabotinsky [46] repeated and extended the work of Belousov, substituting citric acid with another organic compound, malonic acid. He also noticed that the cerium ions could be replaced by ferroin to give a more dramatic red-blue color change compared to the pale yellowcolorless change for the cerium catalyzed system. This reaction has now come to be referred to as the Belousov-Zhabotinsky (BZ) reaction.

In its classical form, the $\mathrm{BZ}$ reaction involves the acidic bromate oxidation of an organic acid in the presence of a metal ion catalyst, where the overall reaction is $[47,48]$ :

$$
3 \mathrm{BrO}_{3}^{-}+5 \mathrm{CH}_{2}(\mathrm{COOH})_{2}+3 \mathrm{H}^{+} \rightarrow 3 \mathrm{BrCH}(\mathrm{COOH})_{2}+2 \mathrm{HCOOH}+4 \mathrm{CO}_{2}+5 \mathrm{H}_{2} \mathrm{O} \quad 7.2
$$


Bromate appears to be a necessary ingredient of the BZ reaction [49]. All of the other constituents, however, can be replaced without altering the qualitative features of the system. Aside from $\mathrm{Ce}^{2+}$ as the catalyst, it has been demonstrated that $\mathrm{Mn}^{2+}, \mathrm{Fe}(\mathrm{phen})_{3}{ }^{2+}$, and $\mathrm{Ru}(\mathrm{bpy}){ }_{3}{ }^{2+}$ can also serve as catalysts $[49,50]$. The reduction potential of a suitable 1equivalent reducing catalyst should be between 1.0 and 1.5 Volt/equiv [49]. A number of organic acids have also been investigated to see whether or not they could generate oscillations [51]. It was found that a suitable substrate was one that could be readily brominated, with the subsequent product being capable of releasing bromide ions when oxidized by the metal catalyst [49]. A strong acid is also an essential ingredient of the BZ reaction and most versions have utilized sulfuric acid. One can use nitric acid in the place of sulfuric acid; however, this increases the frequency of the oscillations [49]. Use of $\mathrm{HCl}$, another strong acid, has proved to be unsuitable, as chloride ions are known to suppress the oscillatory behavior $[49,52]$.

\subsubsection{Effect of Light on the Belousov-Zhabotinsky (BZ) Medium}

The effect of light on the BZ reaction was noted by Vavilin et al. [37] in 1968. They showed that irradiation of the cerium-catalyzed BZ medium with ultraviolet (UV) light could either modify or inhibit the oscillations. Busse and Hess [53] found that UV light could also be used to initiate waves in the ferroin-catalyzed BZ reaction. Gáspár et al. [54] studied the effect of visible light on the BZ system by employing various catalysts and demonstrated that the oscillatory dynamics could either be altered or suppressed altogether. Kuhnert et al. $[45,55]$ demonstrated the use of the photosensitive BZ system as a memory device. 
The ruthenium-catalyzed system has been studied in greater depth over the years owing to the photosensitive properties of the $\left[\mathrm{Ru}(\mathrm{bpy})_{3}\right]^{2+}$ complex [56]. The ruthenium catalyst is sensitive to visible light and is photochemically excited by $460 \mathrm{~nm}$ light. The excited state of the catalyst then reacts with bromomalonic acid to produce bromide ions [57], an inhibitor of autocatalysis, in a series of reactions. This allows the excitability of the system to be adjusted by changing light intensity, with excitability decreasing with increasing light intensity.

\subsubsection{The FKN Mechanism}

To model the BZ reaction, Field, Körös and Noyes (FKN) developed a mechanism in which malonic acid is oxidized by bromate and catalyzed by cerium. This mechanism is also effective in characterizing the qualitative features of the various alterations to the original $\mathrm{BZ}$ reaction.

The reaction mechanism given in Table 7.1 can be divided into three processes. Process A, R1 - R4, involves the formation of bromomalonic acid and consumption of bromide ions through the reduction of bromate via a series of oxygen transfers. R1 is the rate determining step for the overall process. Process B, R5 - R7, which involves the autocatalytic oxidation of metal ion catalyst, becomes important at low bromide concentrations. R5 is the rate determining step for the overall process. The net effect of R5 and R6 is to generate bromous acid autocatalytically, as two molecules of bromous acid are formed for every molecule consumed. Process $\mathrm{C}$, involves the oxidation of bromomalonic acid by the metal catalyst through overall reactions R8 and R9. Process C 
is very complex, with the production of bromide ion and various organic substances being determined by the reaction conditions.

Table 7.1: The Field, Körös and Noyes (FKN) Mechanism

\section{REACTIONS}

Process A: Consumption of Br-and production of BrMA

$$
\begin{gathered}
\mathrm{Br}^{-}+\mathrm{BrO}_{3}^{-}+2 \mathrm{H}^{+} \rightarrow \mathrm{HBrO}_{2}+\mathrm{HOBr} \\
\mathrm{Br}^{-}+\mathrm{HBrO}_{2}+\mathrm{H}^{+} \rightarrow 2 \mathrm{HOBr} \\
3\left(\mathrm{Br}^{-}+\mathrm{HOBr}+\mathrm{H}^{+} \rightarrow \mathrm{Br}_{2}+\mathrm{H}_{2} \mathrm{O}\right) \\
3\left(\mathrm{Br}_{2}+\mathrm{CH}_{2}(\mathrm{COOH})_{2} \rightarrow \mathrm{BrCH}(\mathrm{COOH})_{2}+\mathrm{Br}^{-}+\mathrm{H}^{+}\right) \\
\mathbf{2} \mathbf{B r}^{-}+\mathbf{B r O}_{3}{ }^{-}+\mathbf{3} \mathbf{H}^{+}+\mathbf{3} \mathbf{C H}_{2}(\mathbf{C O O H})_{2} \rightarrow \mathbf{3} \mathbf{B r C H}(\mathbf{C O O H})_{2}+\mathbf{H}_{2} \mathbf{O}
\end{gathered}
$$

Process B: Autocatalytic oxidation of $\mathrm{Ce}^{3+}$

$$
\begin{gathered}
2\left(\mathrm{BrO}_{3}{ }^{-}+\mathrm{HBrO}_{2}+\mathrm{H}^{+} \rightarrow 2 \mathrm{BrO}_{2}{ }^{-}+\mathrm{H}_{2} \mathrm{O}\right) \\
4\left(\mathrm{BrO}_{2}{ }^{-}+\mathrm{Ce}^{3+}+\mathrm{H}^{+} \rightarrow \mathrm{HBrO}_{2}+\mathrm{Ce}^{4+}\right) \\
\mathrm{HBrO}_{2} \rightarrow \mathrm{BrO}_{3}{ }^{-}+\mathrm{HOBr}+\mathrm{H}^{+} \\
\mathbf{B r O}_{3}^{-}+\mathbf{4} \mathbf{C e}^{\mathbf{3 +}}+\mathbf{5} \mathbf{H}^{+} \rightarrow \mathbf{H O B r}+\mathbf{4} \mathbf{C e}^{\mathbf{4 +}}+\mathbf{2} \mathbf{H}_{2} \mathbf{O}
\end{gathered}
$$

Process $C: \mathrm{Ce}^{4+}$ oxidation of BrMA

$$
\begin{gathered}
6 \mathrm{Ce}^{4+}+\mathrm{CH}_{2}(\mathrm{COOH})_{2}+2 \mathrm{H}_{2} \mathrm{O} \rightarrow 6 \mathrm{Ce}^{3+}+\mathrm{HCOOH}+2 \mathrm{CO}_{2}+6 \mathrm{H}^{+} \\
4 \mathrm{Ce}^{4+}+\mathrm{BrCH}(\mathrm{COOH})_{2}+2 \mathrm{H}_{2} \mathrm{O} \rightarrow \mathrm{Br}^{-}+4 \mathrm{Ce}^{3+}+\mathrm{HCOOH}+2 \mathrm{CO}_{2}+5 \mathrm{H}^{+}
\end{gathered}
$$




\subsubsection{Excitable Media}

In a typical excitable system, each element has a single, stationary state that is stable to subthreshold perturbations. Perturbations exceeding a definitive threshold, however, will cause a large excursion in phase space. Following the excitation, a refractory period sets in, where the system is insensitive to further excitations. The system then recovers to return to the original stable, steady state to full excitability.

General reaction-diffusion equations describing an excitable media for a twovariable, activator-inhibitor system can be expressed as

$$
\begin{gathered}
\tau_{u} \frac{\partial u}{\partial t}=D_{u} \nabla^{2} u+f(u, v ; \Phi), \\
\tau_{v} \frac{\partial v}{\partial t}=D_{v} \nabla^{2} v+g(u, v ; \Phi),
\end{gathered}
$$

where $\nabla^{2}$ is the Laplacian operator, $u$ is the activator, $v$ is the inhibitor, and $\mathrm{D}_{u}$ and $\mathrm{D}_{v}$ are the diffusion coefficients for the activator and inhibitor, respectively. $f(u, v ; \Phi)$ and $g(u, v ; \Phi)$ are the nonlinear functions representing the variations in time due to chemical reactions, and $\Phi$ is the control parameter representing, for example, light intensity. $\tau_{u}$ and $\tau_{v}$ represent the characteristic times for the activator and inhibitor, respectively. The variable $u$ is also known as the 'propagator' or 'trigger' variable, and the variable $v$ is also known as the 'recovery' or 'controller' variable [58]. The general two-variable model represented by Eq. 7.3 has been the basis of successful models for reactiondiffusion behavior in chemical, biological, and physical systems [13]. For example, cases where $\mathrm{D}_{v}=0$, in which the inhibitor is not diffusive, are distinctive of waves of neuronal tissues [58,59], and cases where the diffusion coefficients of the two variables are approximately equal are representative of waves in some BZ reactions $[58,60]$. The 
function of the inhibitor is to reduce the activity of the activator, whereas the activator has a dual function, intensifying its own production through autocatalysis and giving rise to the inhibitor.

The functions in Eq. 7.3 describe the local, nonlinear dynamics of the system, and Figure 7.1. shows a qualitative representation of the model. The nullclines $f(u, v ; \Phi)=0$ and $g(u, v ; \Phi)=0$ are $\mathrm{N}$-shaped and monotone, respectively [58]. The steady states are defined as the point(s) where the nullclines intersect $(f(u, v ; \Phi)=g(u, v ; \Phi=0))$, and the system can be bistable, oscillatory, or excitable depending on how the nullclines intersect. In an excitable system, the middle branch of the $u$ nullcline, the region between the minimum and maximum of the nullcline, characterizes the excitability threshold.

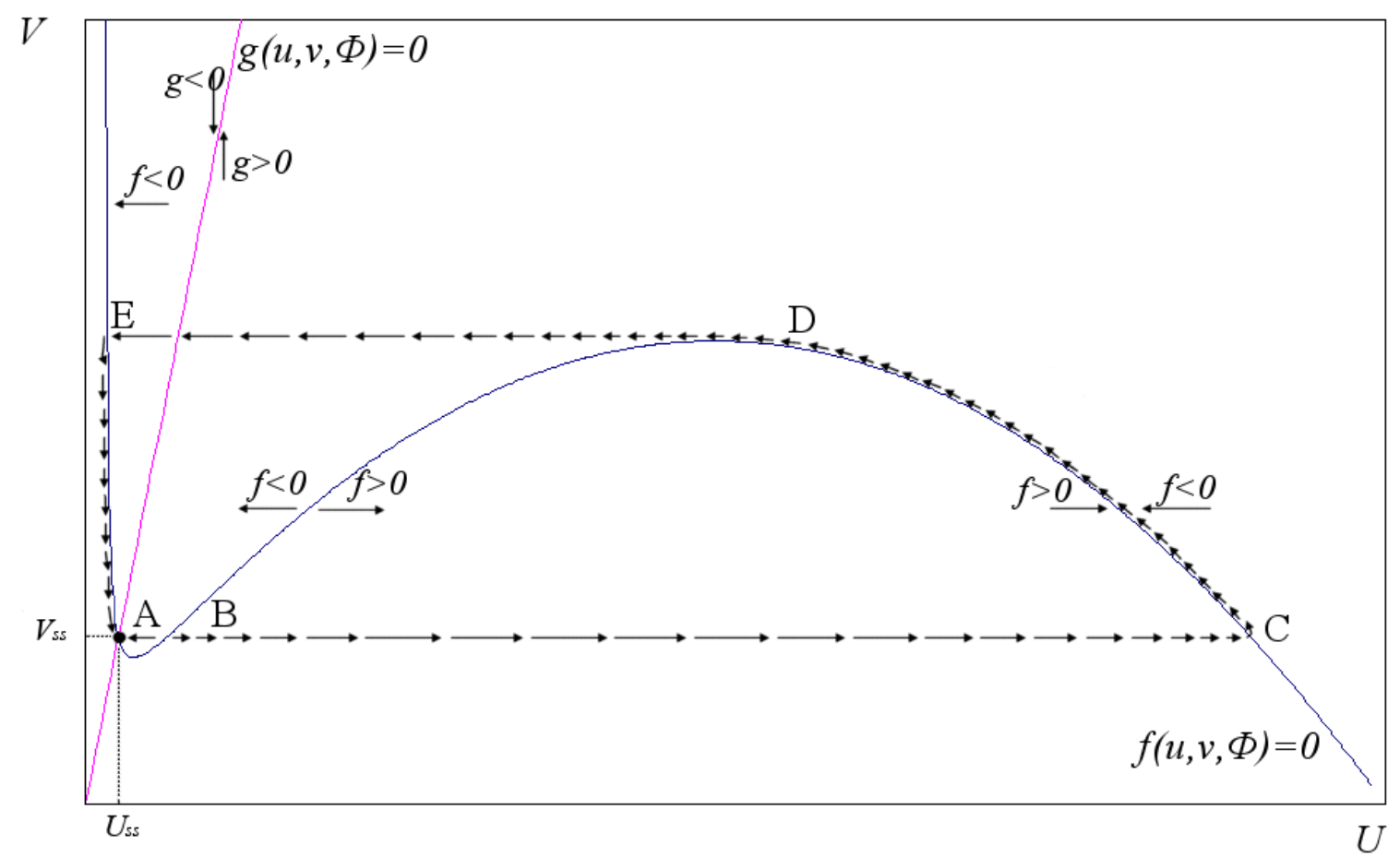

Figure 7.1: Typical phase plane for an excitable system. 
Excitable systems have a number of common features. They possess a stable steady state, $u_{s s}=v_{s s}$, also known as the rest state, and they react in response to perturbations in a similar manner. A subthreshold perturbation, A, in Figure 7.1 to the left of the middle branch, results in a trajectory that decays rapidly to the rest state. A suprathreshold perturbation, B, in Figure 7.1 to the right of the middle branch, produces a trajectory that undergoes a large excursion in the phase plane before the system recovers to full excitability. During the large excursions, the system is refractory and insensitive to further excitations. This series of events is illustrated in the phase plane diagram, Figure 7.1. Typically, the excitable system is defined by three states; the rest state (stable, excitable state), the excited state, and the refractory state (recovery state) and the system can be in any one of these three states at a given time [12].

An active medium is made up of components that are evenly distributed in space and can be locally excited. A variety of types of wave propagation are possible when neighboring components interact through diffusive coupling. Perturbation in a finite subregion in space, beyond the excitability threshold, results in the autocatalytic production of the propagator species $u$ in the region of excitation, resulting in its increase. This gives rise to $u$ spreading into adjacent areas, which causes the neighboring components to exceed the excitability threshold, thus allowing the excitation to continue to spread [58].

In one-dimensional systems, such waves of excitation travel as a wave train of impulses. Two topologically distinct periodic patterns are found for two-dimensional excitable media: spreading homocentric circular waves, also known as target patterns, 
and rotating spiral waves [61]. Spreading homocentric spherical waves and rotating scroll waves are exhibited in three-dimensional systems.

There are three necessary conditions for the initiation of waves and their propagation. First, a steep and substantial response in phase space is required. Second, some form of transport, typically supplied by diffusion, is required for the wave of excitation to spread out in space. Finally, the reaction and diffusion time scales should be comparable to ensure coupling between reaction and diffusion. The role of diffusion is to facilitate the spreading of reactive species, while that of reaction is to transform reactive species into more reactive species and into products. Traveling waves appear as a result of the coupling between reaction and diffusion.

A variety of active media support propagating waves of chemical, physical or biological activity. Examples of active media can be compared according to their state variables, $u$ and $v$, as shown in Table $7.2[58,62]$. Among chemical systems, the BZ reaction has been subjected to more extensive studies, as it is much easier to prepare and control compared to biological systems.

Table 7.2: State variables of some representative excitable media $[58,62]$.

\begin{tabular}{lll}
\hline System & Activator (trigger variable) & Controller (recovery variable) \\
\hline Neuromscular tissue & memberane potential & ionic conductance \\
BZ reaction & bromous acid & oxidized catalyst \\
Dictyostelium discoideum & cyclic AMP & membrane receptor \\
Epidemics & infectious agent & level of immunity \\
Spiral galaxies & density of molecular cloud & temperature of molecular cloud \\
\hline
\end{tabular}




\subsubsection{Resonance}

The dynamics of periodically driven nonlinear systems has been subjected extensive investigations over the years. Resonance-like and synchronization-like phenomena [6365] have been studied in a wide variety of systems [66].

The BZ reaction has been used to study the effect of external periodic forcing on the spatiotemporal dynamics. Petrov et al. [67] were able to transform a rotating spiral wave into a labyrinthine standing wave pattern by periodically forcing the photosensitive BZ medium. The forcing frequencies were related to the oscillation frequency of the independent medium. The reinforcing effects of noise were first reported by Kádár et al. [68], where random fluctuations in light intensity greatly enhanced the propagation of waves in a subexcitabe medium. Müller et al. [69-71] were able to manipulate the dynamics of meandering spiral waves by applying an external periodic force via the imposed light intensity.

All nonlinear physical, chemical and biological oscillators display phase locking (also known as mode or frequency locking) when subjected to an external periodic force. How the oscillator responds to external periodic forcing is largely determined by the forcing parameters, namely the amplitude $\alpha$ and the frequency $\tau$. The corresponding dynamics of the oscillator are either periodic, quasiperiodic or chaotic $[72,73]$.

Usually these driven oscillators display sustained oscillatory behavior even in the absence of external periodic forcing. We consider, on the other hand, a system that is nonoscillatory but close to a subcritical Hopf bifurcation, with a deterministic frequency $\omega$. Hence, when perturbed, the system responds via weak, damped oscillations that decay rapidly to the excitable steady state. Frequency locking occurs at $\lambda=\omega / \tau$ when an 
external periodic force at frequency $\tau$ is applied to the excitable system. Initiation of waves in this case is due to a local resonance between the weak, damped oscillations around the excitable steady state and the external sinusoidal perturbation [32].

If the period between two consecutive waves is rationally related to the period of the sinusoidal perturbation, we show that for every $q$ forcing perturbations, $p$ waves are initiated, i.e., we demonstrate $p: q$ phase locking [74-76]. Thus, a plot of frequency vs. amplitude should reveal domains of firing patterns where phase locking occurs, with $p$ waves within $q$ forcing cycles. These frequency locked domains are known as Arnold tongues [77,78], and their sequence is intimately related to the sequencing of the rational numbers in the Farey series [79]. It is important to note that it is very difficult, if not impossible, to experimentally observe the higher-order tongues, which are typically very narrow. A plot of firing rate or firing number, $p / q$, vs. frequency for a fixed amplitude $\alpha$ has a distinctive form and is known as a devil's staircase [80,81]. The quantity $p / q$ has discrete values within the mode locked regions [82].

In this chapter we present experimental findings and numerical simulations of external periodic forcing on an excitable BZ medium in a thin gel layer. A finite subregion of the photosensitive, reaction-diffusion system is forced periodically with spatially uniform sinusoidal pulses of light in order to study the effect of resonance induced chemical waves. Resonance patterns are found by relating the period between two waves to the period of the sinusoidal perturbation. Numerical simulations were carried out using the modified Oregonator model $[83,84]$, modified to account for the photosensitivity of the $\mathrm{BZ}$ reaction [45,57]. 


\subsection{EXPERIMENTAL SECTION}

Experiments were carried out on a photosensitive Belousov-Zhabotinsky (BZ) medium $[2,55]$. To study the initiation of chemical waves in an excitable system using resonance the medium was illuminated with $460 \mathrm{~nm}$ light by a computer controlled video projector, and the spatiotemporal behavior was monitored with a video camera.

Preparation of Stock Solutions. All stock solutions, with concentrations given in Table 7.3, were prepared using analytical grade chemicals (Fisher), without further purification, and doubly distilled water. They were stored in a refrigerator at a temperature of approximately $3{ }^{\circ} \mathrm{C}$ for later use.

Preparation of Sodium Silicate Solution. A stock solution of 15\% (w/w) sodium silicate was prepared by dissolving $150 \mathrm{~g}$ sodium trisilicate $\left(\mathrm{Na}_{2} \mathrm{Si}_{3} \mathrm{O}_{7}\right)$ in boiling, doubly distilled water. The solution was allowed to boil while stirring until it became transparent. It was then allowed to cool to room temperature and filtered.

Table 7.3: Concentrations of Stock Solutions

\begin{tabular}{ll}
\hline Reagent & Concentration \\
\hline $\mathrm{NaBrO}_{3}$ & $1.5 \mathrm{M}$ \\
$\mathrm{H}_{2} \mathrm{SO}_{4}$ & $3.0 \mathrm{M}$ \\
Malonic Acid & $1.0 \mathrm{M}$ \\
$\mathrm{NaBr}$ & $1.0 \mathrm{M}$ \\
\hline
\end{tabular}


Synthesis of the Ru(bpy) ${ }_{3}{ }^{2+}$ sulfato salt. Bipyridine $(11.4 \mathrm{~g})$ and $\mathrm{Ru}(\mathrm{III}) \mathrm{Cl}_{3}(4.0 \mathrm{~g})$ were refluxed in $95 \%$ ethanol $(100 \mathrm{ml})$ for 72 hours, after which the mixture was filtered to remove excess $\mathrm{Ru}(\mathrm{III}) \mathrm{Cl}_{3}$. Sulfuric acid $(60 \mathrm{ml}$ of $6 \mathrm{M})$ was then added to the filtrate after distilling to approximately half its original volume. The mixture was then left overnight in the freezer. The resultant water soluble crystals were then filtered, washed with ether, and left to dry in a vacuum desiccator. The powder was stored in a refrigerator at approximately $3{ }^{\circ} \mathrm{C}$ for later use.

Preparation of $R u(b p y){ }_{3}{ }^{2+}$ solution. Approximately $0.2 \mathrm{~g}$ of the $\mathrm{Ru}(\mathrm{bpy})_{3}{ }^{2+}$ sulfato salt were dissolved in doubly distilled water. The concentration was then determined accurately spectrophotometrically using its known extinction coefficient of $14,600 \mathrm{M}^{-1}$ $\mathrm{cm}^{-1}$ at $452 \mathrm{~nm}$ [85]. Measurements were carried out using a Hewlett-Packard (HP845x UV-Visible Chemstation) spectrophotometer.

Preparation of Catalyst-free BZ Solution. Appropriate amounts of the stock solutions were mixed to yield a catalyst-free BZ solution with concentrations given in Table 7.4. Fresh solutions were prepared for each experiment and were kept in an ice bath from when they were prepared until the experiment. Bromomalonic acid (BrMA) was generated in situ according the following reaction [86]:

$$
\mathrm{BrO}_{3}{ }^{-}+2 \mathrm{Br}^{-}+3 \mathrm{MA}+3 \mathrm{H}^{+} \rightarrow 3 \mathrm{BrMA}+3 \mathrm{H}_{2} \mathrm{O}
$$


Table 7.4: Composition of catalyst-free BZ solution

\begin{tabular}{ll}
\hline Reagent & Concentration \\
\hline $\mathrm{NaBrO}_{3}$ & $0.552 \mathrm{M}$ \\
$\mathrm{H}_{2} \mathrm{SO}_{4}$ & $0.4891604 \mathrm{M}$ \\
Malonic Acid & $0.026 \mathrm{M}$ \\
BrMA & $0.162 \mathrm{M}$ \\
\hline
\end{tabular}

Preparation of gel. Ruthenium(II)-bypyridyl (Ru(bpy) $\left.{ }_{3}{ }^{2+}\right)$, the light-sensitive catalyst of the BZ reaction, was immobilized in a silica gel medium [87] $(0.3 \times 20 \times 20 \mathrm{~mm})$, prepared by acidifying a solution of $15 \%(\mathrm{w} / \mathrm{w}) \mathrm{Na}_{2} \mathrm{Si}_{3} \mathrm{O}_{7}$ and $1.88 \mathrm{mM} \mathrm{Ru}(\mathrm{bpy})_{3}{ }^{2+}$ with 1.2 $\mathrm{M} \mathrm{H}_{2} \mathrm{SO}_{4}$ and cast uniformly onto a microscope slide. A new gel containing ruthenium(II)-bipyridyl was prepared for each experiment.

\subsubsection{Experimental Setup}

Figure 7.2 shows the main setup of the experiment. This setup is comprised of the $\mathrm{BZ}$ reaction gel and reactor, and the instrumentation to record the observed results.

The microscope slide, onto which the gel containing the catalyst is cast (stripped rectangle within the reactor in Figure 7.2), was mounted in a thermostatic reactor face up. The gel was continuously bathed with fresh catalyst-free BZ solution, so as to maintain a constant nonequilibrium state [10]. The reaction was carried out in an open rector at 20.0 ${ }^{\circ} \mathrm{C}$ with catalyst-free $\mathrm{BZ}$ solution, maintained at $0{ }^{\circ} \mathrm{C}$ using an ice-bath to prevent decomposition. The solution was continuously pumped to the reactor at a flow rate of 5.0 $\mathrm{ml} / \mathrm{min}$ using a peristaltic pump (Ismatec) through a degasser (Uniflow Degasys Ultimate 
DV 4010). To maintain the thickness of the layer of solution in the reactor constant during the experiment, the reaction products were removed at the same rate.

Computer generated images were projected onto gel medium using a modified video projector (see figure 7.3). The beam of light was filtered through a $460 \mathrm{~nm}$ bandpass filter, $\Delta \lambda=10 \mathrm{~nm}$ (Filt. in Figure 7.2), which is close to the excitation wavelength of ruthenium(II)-bipyridyl [56]. Positioned between the microscope slide and the bottom of the reactor was a white membrane (Pall Gelman Laboratory, supor ${ }^{\circledR}-450,0.45 \mu \mathrm{m}, 90$ $\mathrm{mm}$ ) from which light from the projector was scattered. The membrane also serves as a reflective background for imaging the chemical waves. Images were captured using a video camera (Cam. 1 in Figure 7.2) fitted with a macro lens, and were processed by a computer in real time. A reference video camera (Cam. 2 in Figure 7.2) was used to make adjustments to the projected image to ensure spatial homogeneity in the illumination. The reduction of innate spatial variations and temporal instabilities of the projector's response were reduced to acceptable levels with the reference camera.

The beam splitter matches the observation and illumination angles so as to combine the observation and illumination optical paths. The beam of light from the video projector is split into two: a reference image on the diffusive screen (Scr. in Figure 7.2) is produced by light passing through the beam splitter, while the reflected light travels to the gel. A pair of crossed polarizers (Pol. 1 and Pol. 2 in Figure 7.2) reduces the amount of light reflected from the reference screen to the primary camera. To produce the same size images, distances along the optical axis from the beam splitter to the reference screen and to the gel are made equal. The primary camera is positioned above the beam splitter in such a way to ensure that its optical axis coincides with the path of light between the 
beam splitter and the gel. To ensure that the projected images are obtained with similar scaling, the reference camera is positioned at the same distance from the reference screen, as the primary camera is from the gel. To avoid reflections from the solution surface, most of the plane optical surfaces in the system are tilted by a small angle from the perpendicular (not shown in Figure 7.2).

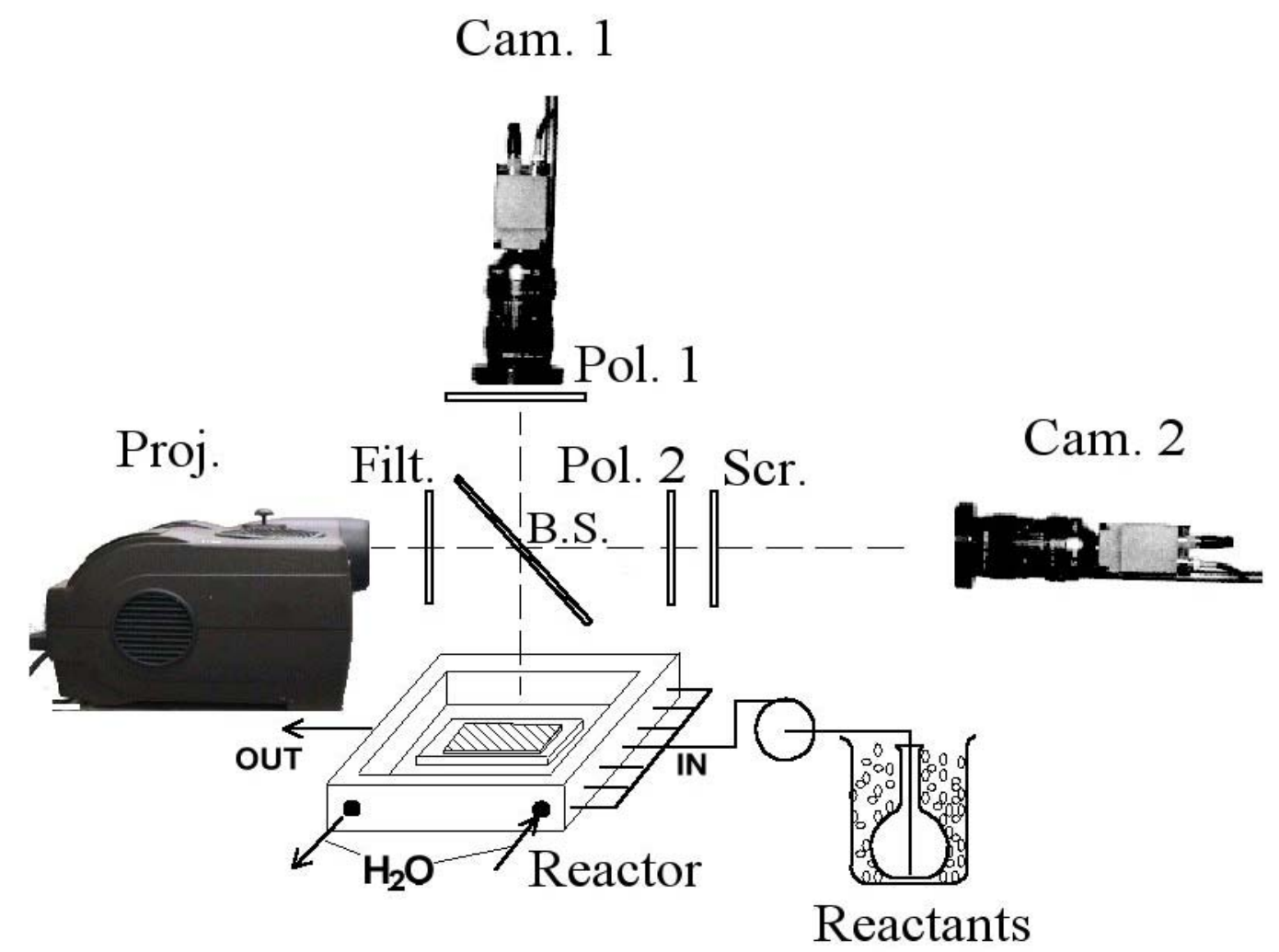

Figure 7.2: Illustration of experimental setup. See text for details.

\subsubsection{Resonance}

Analyzing the dynamics of the system systematically as a function of light intensity $\varnothing$ showed that the system is oscillatory for $\varnothing<8.80 \times 10^{-2} \mathrm{~mW} / \mathrm{cm}^{2}$ and excitable for $\emptyset>$ 
$8.80 \times 10^{-2} \mathrm{~mW} / \mathrm{cm}^{2}$. It should be noted that in all experiments, the domain of oscillatory behavior was deliberately excluded by making certain that the control variable $\varnothing>\emptyset_{\text {osc }}$ where $\emptyset_{\text {osc }}=8.80 \times 10^{-2} \mathrm{~mW} / \mathrm{cm}^{2}$.

To carry out the experiments, an illumination profile produced by the video projector, as shown in Fig. 7.3, was projected onto the gel medium. The boundary region (white) was maintained at high light intensities to prevent wave propagation beyond the reaction zone (gray). In the reaction zone, light intensity was maintained at $\varnothing=4.85 \times 10^{-}$ ${ }^{1} \mathrm{~mW} / \mathrm{cm}^{2}$, thus ensuring that the system was in the excitable domain where initiation of chemical waves is possible. Waves were initiated in the center region (dark gray) by periodically varying the light intensity according to the following relation [32]:

$$
\varnothing=\emptyset_{0}\left[1+\alpha \sin \left(\frac{2 \pi t}{\mathrm{~T}}\right)\right]
$$

The light intensity at the center region, consisting of a 60 cell x 60 cell array, was adjusted at $1.0 \mathrm{~s}$ intervals.

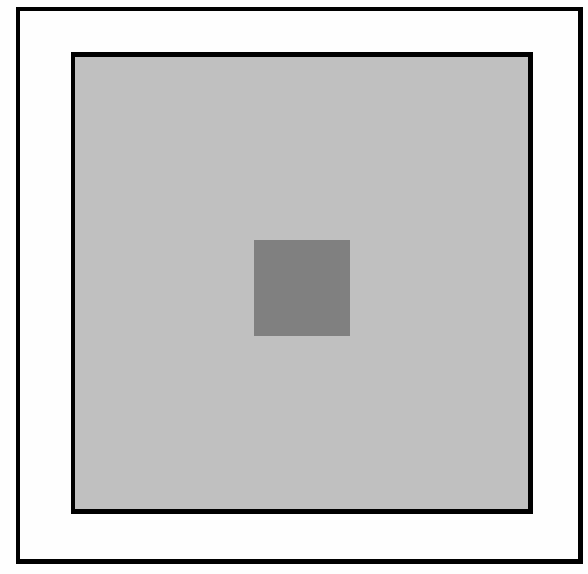

Figure 7.3: Image of the illumination pattern projected onto the gel. 


\subsubsection{Experimental Results}

Systematically scanning the forcing period and forcing amplitude, close to the bifurcation point, defined the region in parameter space where waves can be initiated using resonance. Figure 7.5 shows the frequency-amplitude domain where complex nonlinear resonance [82] between the excitable system and the sinusoidal perturbation results in the initiation of waves [32]. No waves are initiated in the region below the Vshaped curve, and above the optimal amplitude $(\alpha=0.14)$, initiation of waves becomes trivial, as the system then crosses the bifurcation point into the oscillatory region.

An example of resonance induced chemical waves is illustrated in Figure 7.4, where panel (a) shows the initially quiescent excitable medium. In the absence of external periodic modulations in the light intensity, no waves are initiated. Periodic variations in light intensity at the center (dark) region of the medium results in waves being initiated over a certain range of forcing amplitude and forcing period, as shown in Figure 7.5. The wave initiated in panel (b) propagates outward in the form of a circular wave, as shown in panels (c) - (e). Panel (f) shows another wave being initiated. In this particular case, the period between two waves is the same as the period of the perturbation, i.e., a wave is initiated for every perturbation giving rise to a 1:1 resonance pattern. We can also quantify such patterns in terms of the firing number $f n$, which is defined as the ratio between the number of waves initiated $p$ to the number of forcing cycles $q$. Thus, for the parameters corresponding to Figure 7.4 we find $f n=1.0$.

A bifurcation diagram can be constructed showing the relationship between firing number and the period of the sinusoidal perturbation by systematically varying the period of the sinusoidal wave for a fixed forcing amplitude $\alpha$. Figure 7.6 shows that such a 
diagram has a distinctive form, commonly referred to as the devil's staircase $[80,82,88]$, which is typical for forced excitatory behavior [32]. A wave is initiated for each and every perturbation when the period of the perturbation is greater than $66 \mathrm{~s}$, as system will have relaxed back to the excitable state from the previous excitation to support another wave initiation. The result is a 1:1 resonance with a firing number $f n=1.0$. If the period of perturbation is decreased to $56 \mathrm{~s}$, a change in firing pattern takes place from a 1:1 resonance pattern to a 2:3 resonance pattern, $f n=0.66$, where two waves are initiated for every three forcing cycles. Decreasing the period of perturbation even further to $26 \mathrm{~s}$ results in a change of the resonance pattern to the firing number $f n=0.5$. In this case, a wave is initiated every other forcing cycle, as the system can not recover from the previous excitation to sustain another wave, i.e., the system remains in the refractory stage. Decreasing the period of perturbation even further to $13 \mathrm{~s}$ results in a new pattern, with the firing number $f n=0.33$, where one wave is initiated every three forcing cycles. When the period of perturbation is $7 \mathrm{~s}$, the firing pattern now has a firing number of $f n=$ 0.25 , where one wave is initiated every four cycles. For lower amplitudes, using the reaction parameters as given in Figure 7.4, one observes only 1:1 and 1:2 resonances. One might expect higher resonances, for example, 2:5 or 2:7 resonance patterns between the simple resonance patterns $1: 2$ and $1: 3$ or $1: 3$ and $1: 4$, respectively, but we were unable to find these in our experiments. Such patterns usually occupy very narrow regions and were not detectable with our experimental apparatus. 

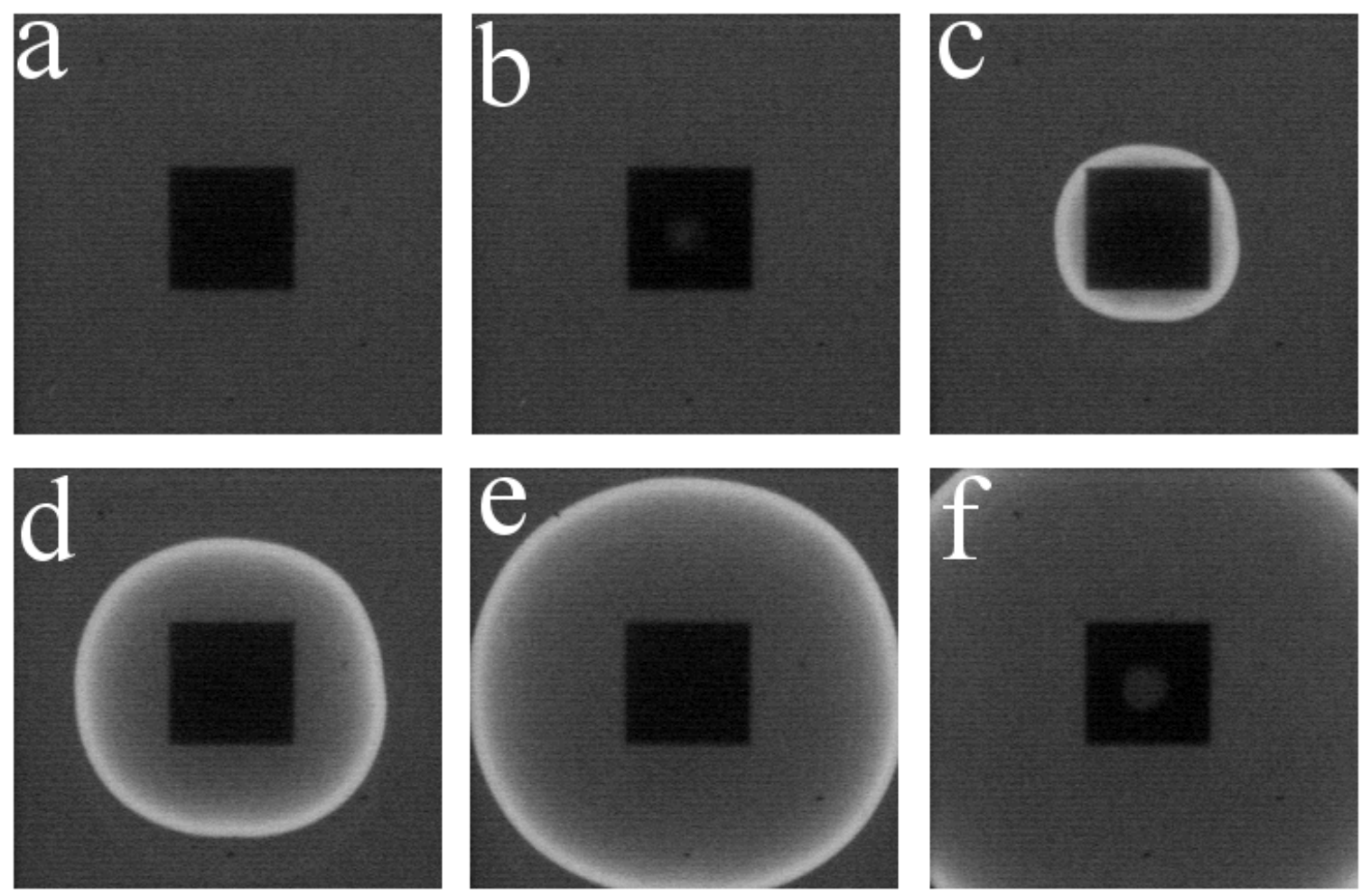

Figure. 7.4: Images of an oxidation wave (white) propagating into an excitable light sensitive BZ medium, collected at equal time intervals of $t=20.0 \mathrm{~s}$. Panels show the initiation of a chemical wave in an initially quiescent media via localized (dark square) periodic modulations of light intensity: $I=I_{\mathrm{o}}[1+\alpha \sin (2 \pi v t / \mathrm{T})]$, where $I_{\mathrm{o}}=0.119 \mathrm{~mW} / \mathrm{cm}^{2}, \alpha=0.10$, and $\mathrm{T}=80 \mathrm{~s}$. In the reaction zone, the light intensity was maintained at $\varnothing=0.485 \mathrm{~mW} / \mathrm{cm}^{2}$. (a) A localized periodic modulation in light intensity is applied to the center of the medium with a spatial size of $60 \times 60$ grid points. (b) Inception of a circular wave, which propagates outward in time, (c) - (e). A wave is initiated every perturbation, resulting in a 1:1 resonant pattern. The reaction was carried out in an open reactor at $20.0{ }^{\circ} \mathrm{C}$, which was continuously supplied with fresh catalyst-free BZ solution (0.552 $\mathrm{M} \mathrm{NaBrO}_{3}, 0.026 \mathrm{M}$ malonic acid, $0.162 \mathrm{M}$ bromomalonic acid, $0.4891604 \mathrm{M} \mathrm{H}_{2} \mathrm{SO}_{4}$ ) maintained at $0.0{ }^{\circ} \mathrm{C}$ to prevent decomposition. Ruthenium(II)-bipyridyl, a light sensitive catalyst, was immobilized in a silica gel medium $\left(0.3 \times 20 \times 20 \mathrm{~mm}^{3}\right)$ prepared by acidifying a solution of $15 \%$ (w/w) $\mathrm{Na}_{2} \mathrm{Si}_{3} \mathrm{O}_{7}$ and $1.88 \mathrm{mM} \mathrm{Ru}(\mathrm{bpy})_{3}{ }^{2+}$ with $\mathrm{H}_{2} \mathrm{SO}_{4}$ and cast onto a microscope slide. Light from the video projector passed through a bandpass filter with a center wavelength of 460 $\mathrm{nm}$. Images of the chemical waves were monitored using a video camera. 


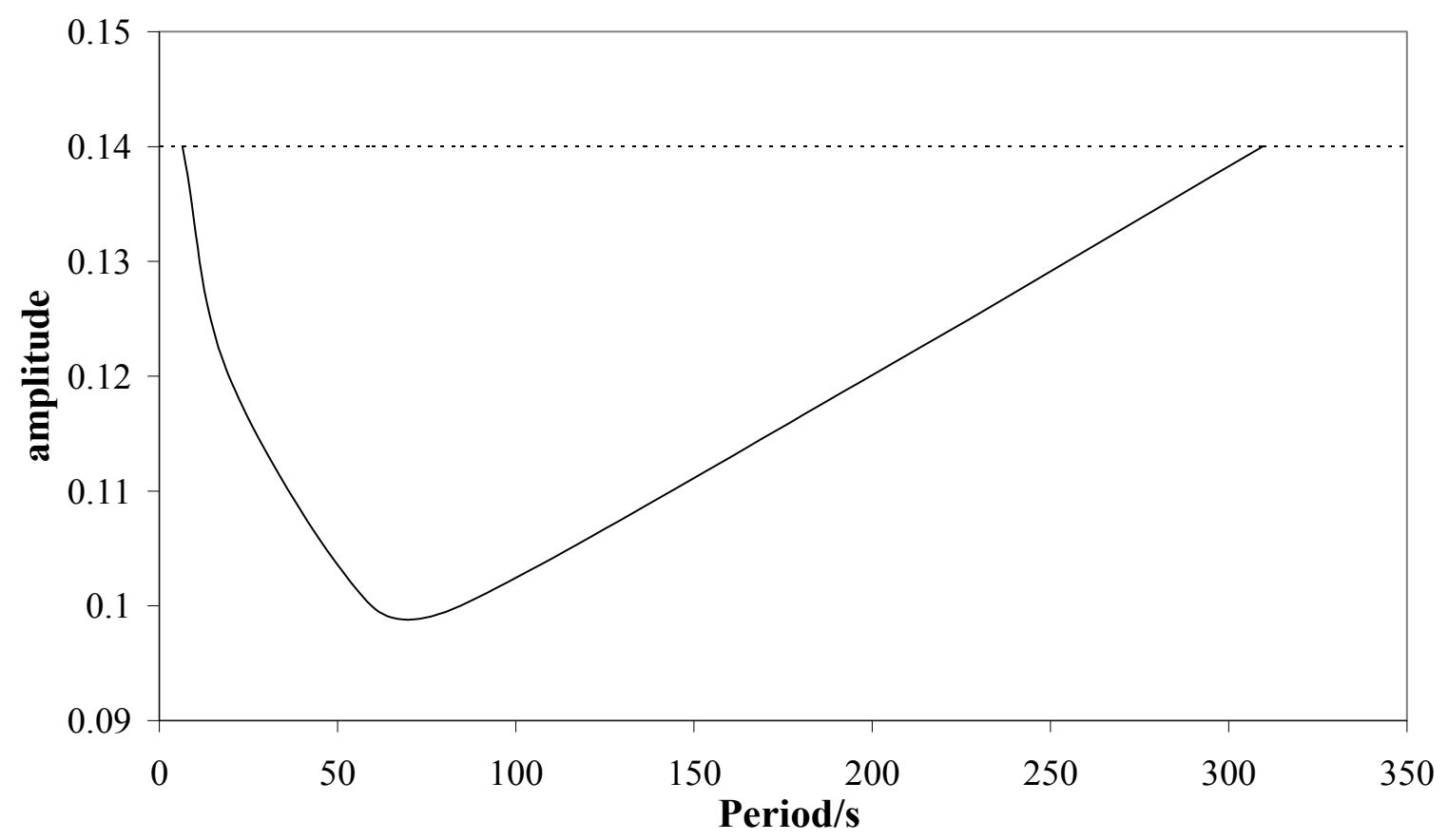

Figure 7.5: V-shaped curve that encloses the region in forcing amplitude - forcing frequency where the initiation of chemical waves is possible with subthreshold variations in light intensity. Parameters are the same as in Figure 7.4. The frequency and amplitude of the applied sinusoidal perturbations were systematically varied in the domain shown. The amplitude $\alpha=0.14$ represents the maximum parameter value that guarantees $\varnothing>\varnothing_{\text {osc }}$, where $\varnothing_{\text {osc }}=8.80 \times 10^{-2} \mathrm{~mW} / \mathrm{cm}^{2}$. 


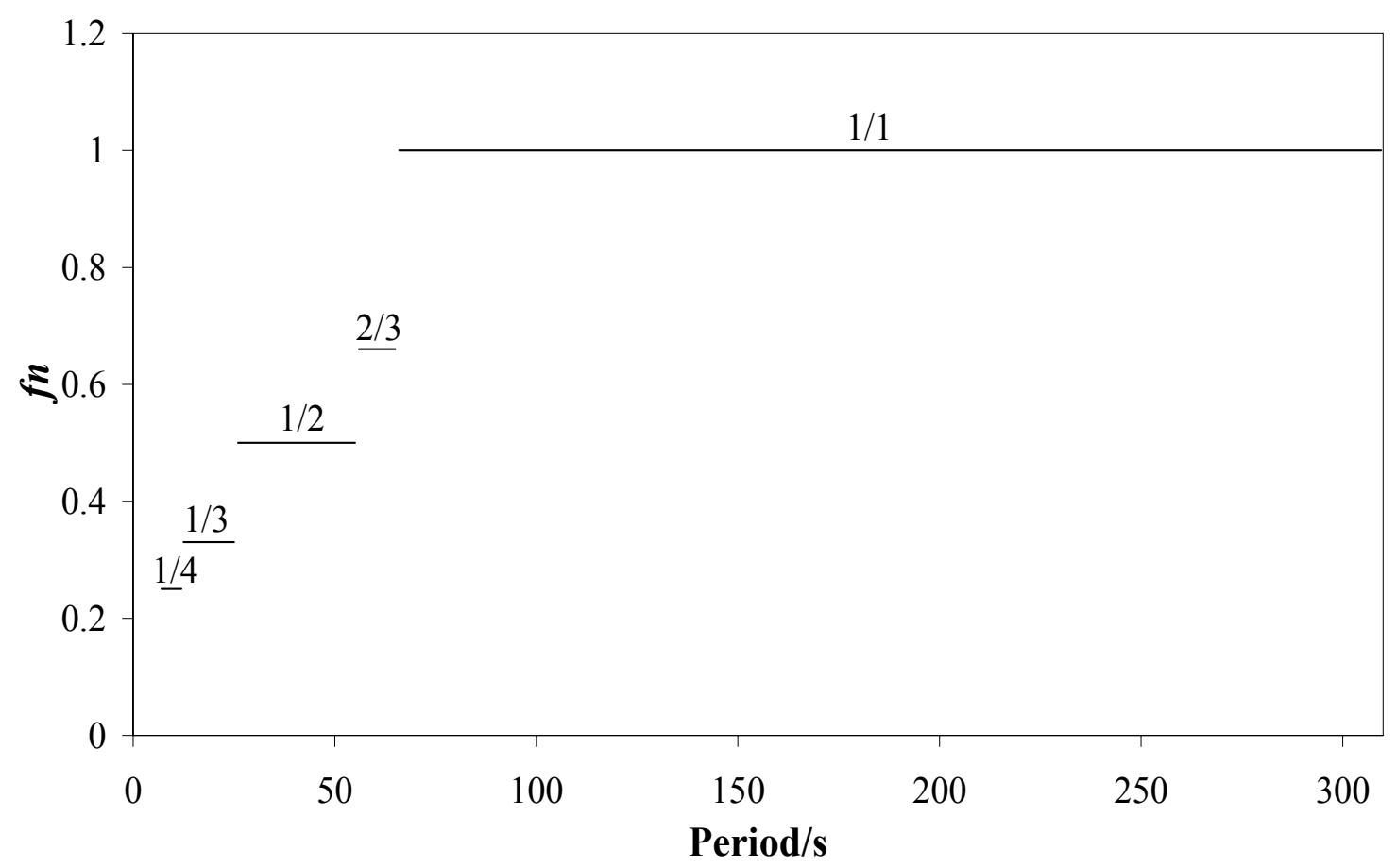

Figure 7.6: Bifurcation diagram showing firing number $f n$ as a function of forcing period for $\alpha=$ 0.14. Parameters are the same as in Figure 7.4, except that forcing amplitude is fixed at $\alpha=0.140$.

\subsection{NUMERICAL SIMULATIONS}

To complete our study of resonance induced chemical waves, numerical investigations were carried out using an Oregonator model $[83,84,89]$ modified to incorporate the photosensitivity of the ruthenium catalyzed BZ reaction $[45,57,68,90]$. Simplifying the model using the Tyson scaling [60,91] enables the spatiotemporal behavior to be described by the following partial differential equations $[32,84]$

$$
\begin{aligned}
& \varepsilon \delta_{\tau} x=x(1-x)+y(q-x)-\varepsilon K_{\mathrm{f}} x+p_{2} \varnothing+\varepsilon \mathrm{D}_{\mathrm{x}} \nabla^{2} x \\
& \varepsilon^{\prime} \delta_{\tau} y=2 h z-y(q+x)+\varepsilon^{\prime} \mathrm{K}_{\mathrm{f}}\left(\mathrm{y}_{0}-\mathrm{y}\right)+p_{1} \varnothing+\varepsilon^{\prime} \varepsilon \mathrm{D}_{\mathrm{y}} \nabla^{2} y \\
& \delta_{\tau} \mathrm{z}=\mathrm{x}-\mathrm{z}-\mathrm{K}_{\mathrm{f}} z+\left(\frac{p_{1}}{2}+p_{2}\right) \varnothing+\mathrm{D}_{\mathrm{z}} \nabla^{2} z
\end{aligned}
$$


where $x, y$, and $z$ are the dimensionless concentration variables related to the actual concentration variables by

$$
x=\frac{2 k_{04}}{k_{03} A} X, y=\frac{k_{02}}{k_{03} A} Y, z=\frac{k_{04} k_{05} M}{\left(k_{03} A\right)^{2}} Z
$$

$\tau$ is the dimensionless time related to the actual time by

$$
\tau=k_{05} M t
$$

The dimensionless parameters $\varepsilon, \varepsilon^{\prime}, q, y_{0}, K_{\mathrm{f}}, \varnothing, p_{1}$, and $p_{2}$ are given by

$$
\begin{aligned}
& \varepsilon=\frac{k_{05} M}{k_{03} A}, \varepsilon^{\prime}=\frac{2 k_{04} k_{05} M}{k_{02} k_{03} A}, q=\frac{2 k_{01} k_{04}}{k_{02} k_{03}}, \\
& y_{0}=\frac{k_{02}}{k_{03} A} Y_{0}, K_{\mathrm{f}}=\frac{1}{k_{05} M} k_{f}, \varnothing=\frac{2 k 04}{(k 03 A)^{2}} \Phi \\
& p_{1}=\frac{V}{0.89+V+15 H^{2} A}, p_{2}=\frac{15 H^{2} A}{0.89+V+15 H^{2} A}
\end{aligned}
$$

The variables $\mathrm{x}, \mathrm{y}$ and $\mathrm{z}$ represent the dimensionless concentrations of bromous acid, bromide, and the oxidized form of catalyst, respectively. The parameter $\varnothing$ is the light intensity that controls the excitability of the medium. Since the Ru (III) complex is immobilized in the silica gel matrix, the corresponding diffusion coefficient is zero, $\mathrm{D}_{z}=$ 0.

\subsubsection{Resonance induced chemical waves}

One- and two-dimensional numerical simulations of Eqs. 7.6 were carried out using the explicit Euler method with zero-flux boundary conditions. A three-point 
approximation for the Laplacian operator $\left(\nabla^{2}=\partial^{2} / \partial x^{2}\right)$ was used for the onedimensional configuration. For the two-dimensional configuration, a five-point approximation for the Laplacian operator $\left(\nabla^{2}=\partial^{2} / \partial x^{2}+\partial^{2} / \partial y^{2}\right)$ was employed. A system size of $1.0 \mathrm{~cm}^{2}$, divided into 50 grid elements, was chosen for the simulation.

The three-variable system (with $D_{i}=0$ ), was subjected to numerical integration and a linear stability analysis. Studying the dynamics of the system in relation to the light intensity $\varnothing$ showed the presence of a subcritical Hopf bifurcation [32,84]. The system was found to be oscillatory for $\varnothing<8.0 \times 10^{-7}$, bistable with a stable limit cycle and a stable focus for $8.0 \times 10^{-7}<\varnothing<8.05 \times 10^{-7}$, and steady state for $\varnothing>\emptyset_{\mathrm{c}}$, where $\emptyset_{\mathrm{c}}=8.05$ $\mathrm{x} 10^{-7}$

To initiate resonance-induced chemical waves, the excitable medium was made nonoscillatory by fixing the excitability with $\varnothing>\emptyset_{\text {c. }}$. Small but finite periodic modulations in light intenstity were then applied to a defined subdivision of the medium according to [32]

$$
\varnothing=\emptyset_{0}\left[1+\alpha \sin \left(\frac{2 \pi t}{\mathrm{~T}}\right)\right]
$$

\subsubsection{Numerical Results}

Systematically varying the forcing amplitude and forcing frequency close to the bifurcation point allows the domain of resonance induced waves to be determined. Figure 7.7 shows the frequency-amplitude domain were complex nonlinear resonances [82] between the damped oscillations around the stable focal steady state and the sinusoidal perturbation results in excitation [32]. Shown within the V-shaped curve are regions were 
the simple resonances 1:1, 1:2, 1:3 and 1:4 occur. These regions are commonly referred to as Arnold tongues [77,78], and their sequence is the same as the sequencing of rational numbers in the Farey series [72,79]. Each region represents a nonlinear resonance at a frequency near that of the imaginary component of the eigenvalues at the subcritical Hopf bifurcation of the autonomous system (Eq. 7.6) [32].

Outside the regions of simple resonance are regions of higher resonances. Figure 7.8 shows a plot of firing number $f n$ versus normalized frequency $v / v_{0}$ for a fixed amplitude $\alpha$ $=0.170$ and reveals the regions where such complex resonances occur. Complex resonances can be observed between the simple resonances, for example, between 1:1 and 1:2 firing patterns can be arranged as a branch of a Farey tree $[92,93]$. This sequence can be generated with any pair of resonance patterns using Farey arithmetic, for example, the daughter pattern of the $a: b$ and $c: d$ patterns is $(a+c) /(b+d)$ [94], as shown in Figure 1.9. As in the experiments, the firing number decreases with increasing forcing frequency. There is a gap between the 1:3 and 1:4 resonances, in which the number of waves initiated and the number of forcing cycles are not rationally related and it is likely that complex patterns occur within this region.

Figure 7.10 and Figure 7.11 display the induced concentration profiles of the variable $x$ and that of the perturbing sinusoidal functions for a fixed amplitude $\alpha=0.16$. Figure 7.10 shows the simple resonances, where a wave is initiated every perturbation in (a), while for (b), a wave is initiated only every other perturbation. In (c), a wave is initiated every third forcing cycle, while in (d), a wave is initiated every fourth forcing cycle. Changes in the firing numbers are due to an increase in forcing frequency. Figure 7.11 reveals some of the higher resonances observed in the system: (a) Five waves are initiated 
for every eleven forcing cycles; (b) four waves are initiated for every nine forcing cycles; (c) three waves are initiated for every seven forcing cycles; and (d) two waves are initiated for every five forcing cycles.

Figure 7.12 shows the simulated spatiotemporal images for resonance induced chemical waves in a two-dimensional excitable system. Sinusoidal perturbations in light intensity are applied locally to an initially homogeneous state, (a). This leads to an excitation, resulting in the initiation of a wave, (b). The wave propagates outward as a traveling circular wave, (c) - (e). For the given model parameters, a wave is imitated every perturbation cycle resulting in a 1:1 resonance pattern. 


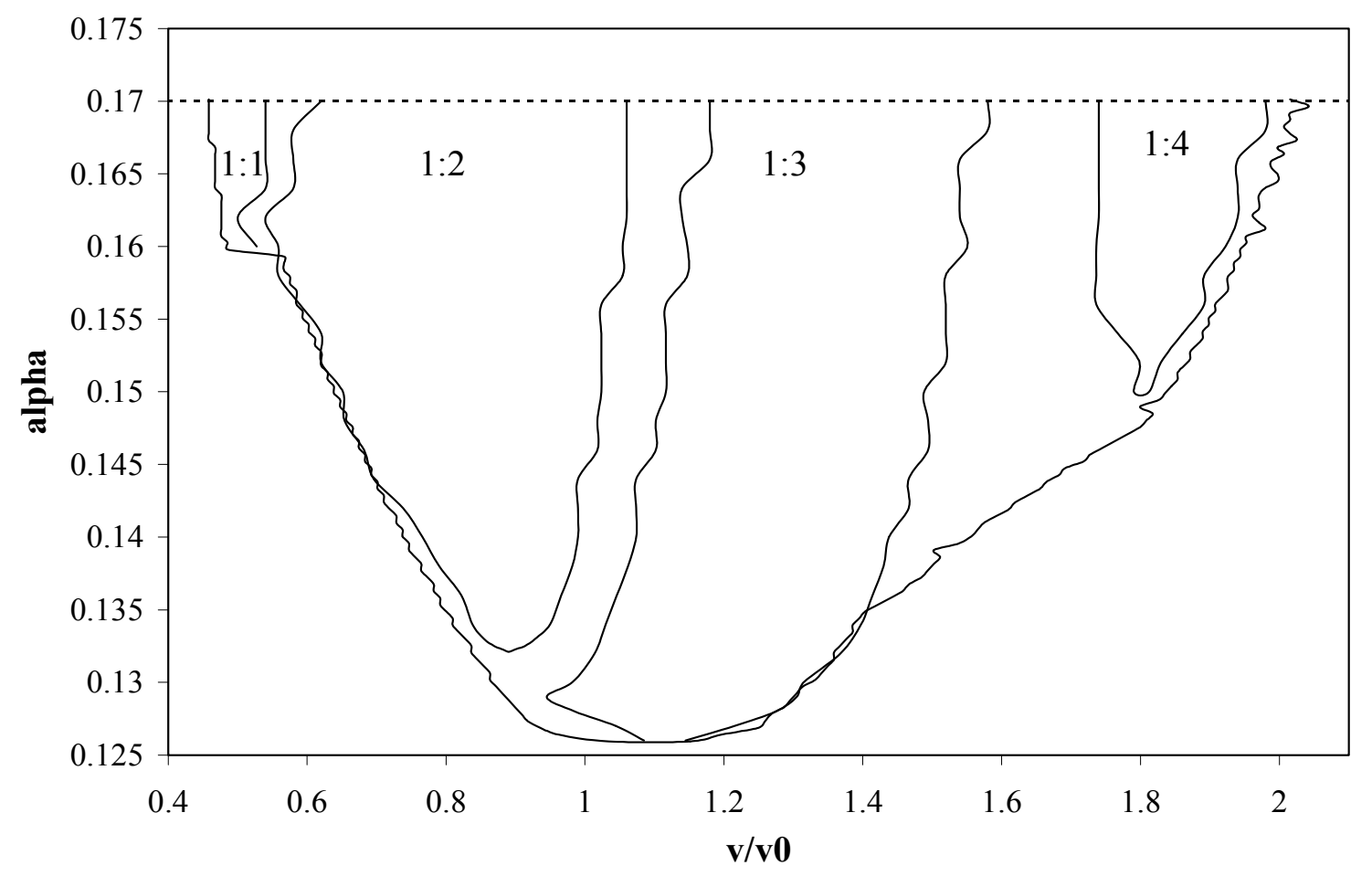

Figure 7.7: Phase diagram showing values of parameters $v / v_{0}$ and $\alpha$ that lead to initiation of waves (region enclosed by the outer V-shaped curve). Shown within the V-shaped curve are mode-locking regions (Arnold tongues) and for clarity, only the 1:q (one wave for $q$ perturbations) are displayed. The simulation was carried out using a one-dimensional lattice of 50 grid points $(\Delta x=0.02, \Delta t=0.0001)$. The parameter $\varnothing$ (light intensity) was maintained at $9.7 \times 10^{-}$ ${ }^{7}$ everywhere in space except at the center where it was adjusted at equal time intervals according to $\varnothing=\varnothing_{0}\left[1+\alpha \sin (2 \pi v \mathrm{t})\right.$, where $\varnothing_{0}=9.7 \times 10^{-7}$. Model parameters of the system are: $k_{01}=\left(2 \mathrm{M}^{-3}\right.$ $\left.\mathrm{s}^{-1}\right) \mathrm{H}^{2}, k_{02}=\left(3 \times 10^{6} \mathrm{M}^{-2} \mathrm{~s}^{-1}\right) \mathrm{H}, k_{03}=\left(42 \mathrm{M}^{-2} \mathrm{~s}^{-1}\right) \mathrm{H}, k_{04}=3 \times 10^{3} \mathrm{M}^{-1} \mathrm{~s}^{-1}, k_{05}=5 \mathrm{M}^{-1} \mathrm{~s}^{-1}, k_{f}=1.05 \mathrm{x}$ $10^{-3} \mathrm{~s}^{-1}, v_{0}=0.02 \mathrm{~s}^{-1}, h=0.5, \mathrm{H}=0.37 \mathrm{M}, A=0.15 \mathrm{M}, M=0.2 \mathrm{M}^{-1}$, and $V=0.05 \mathrm{M}$. Initial conditions: $X_{0}=Z_{0}=0 \mathrm{M}, Y_{0}=1 \times 10^{-4} \mathrm{M}$. Diffusion parameters: $D_{x}=1.5 \times 10^{-5}, D_{y}=1.68 \times 10^{-}$ ${ }^{5}$, and $D_{z}=0$. The parameter threshold that insures $\varnothing>\varnothing_{\mathrm{c}}$, where $\varnothing_{\mathrm{c}}=8.05 \times 10^{-7}$ [32], is satisfied by the value $\alpha=0.1701$. 


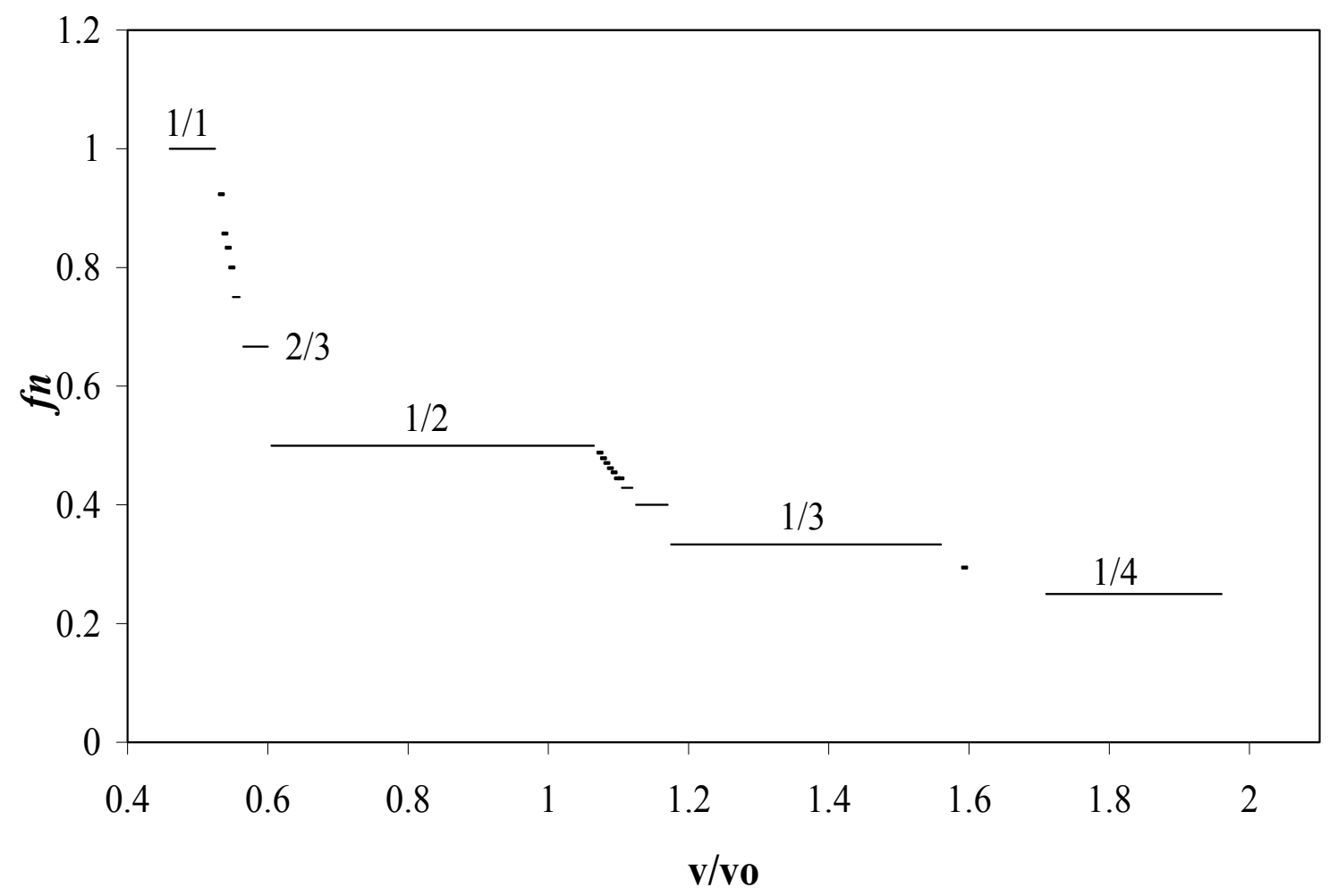

Figure 7.8: Bifurcation diagram showing firing number $f n$ as a function of normalized forcing frequency $v / v_{0}$ illustrating the dominance of 1:q mode locking regions. Model parameters are the same as in Figure 7.7, except that forcing amplitude is fixed at $\alpha=0.170$. 


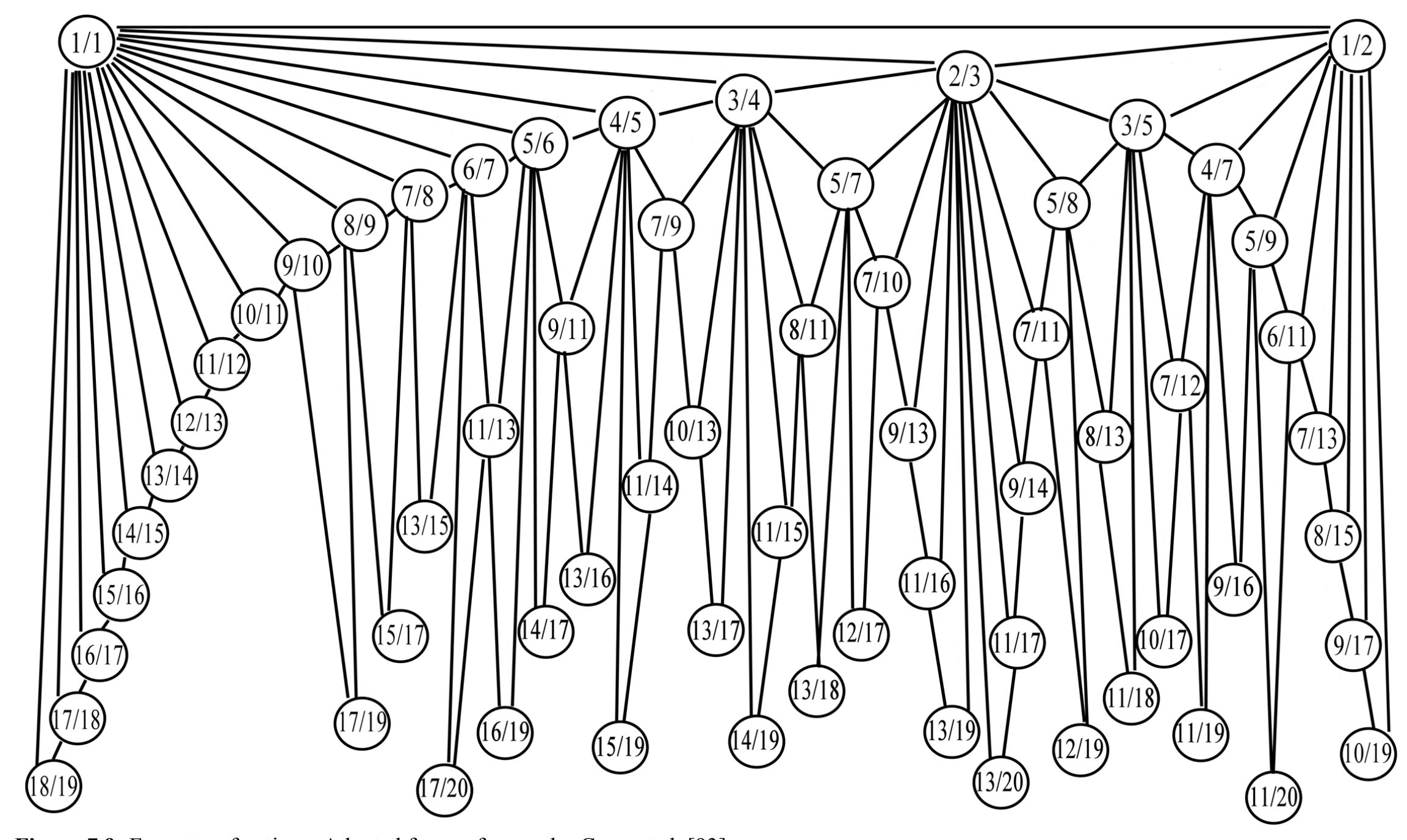

Figure 7.9: Farey tree fractions. Adapted from reference by Coon et al. [93]. 

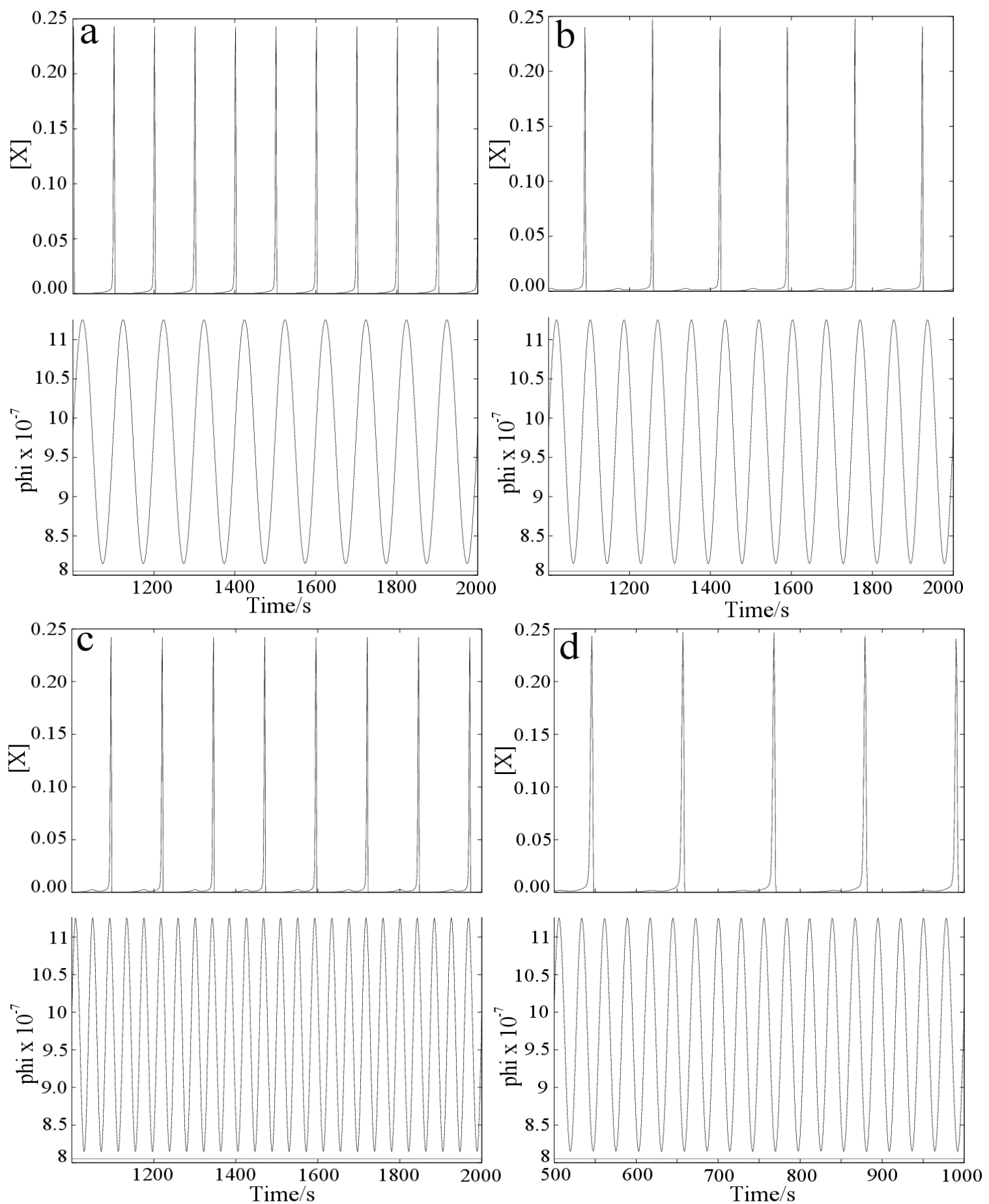

Figure 7.10. Simulated phase locking patterns for $\alpha=0.16$. In all four figures, the upper trace shows the time series of variable $x$, and the lower one is the time series of the sinusoidal perturbation, which clearly does not cross the threshold (bottom line). Model parameters are the same as in Figure 7.7. 1:1 mode locking for $v / v_{0}=0.50$ (a), 1:2 mode locking for $v / v_{0}=0.60$ (b), $1: 3$ mode locking for $v / v_{0}=1.20(\mathrm{c})$, and 1:4 mode locking for $v / v_{0}=1.80(\mathrm{~d})$. 

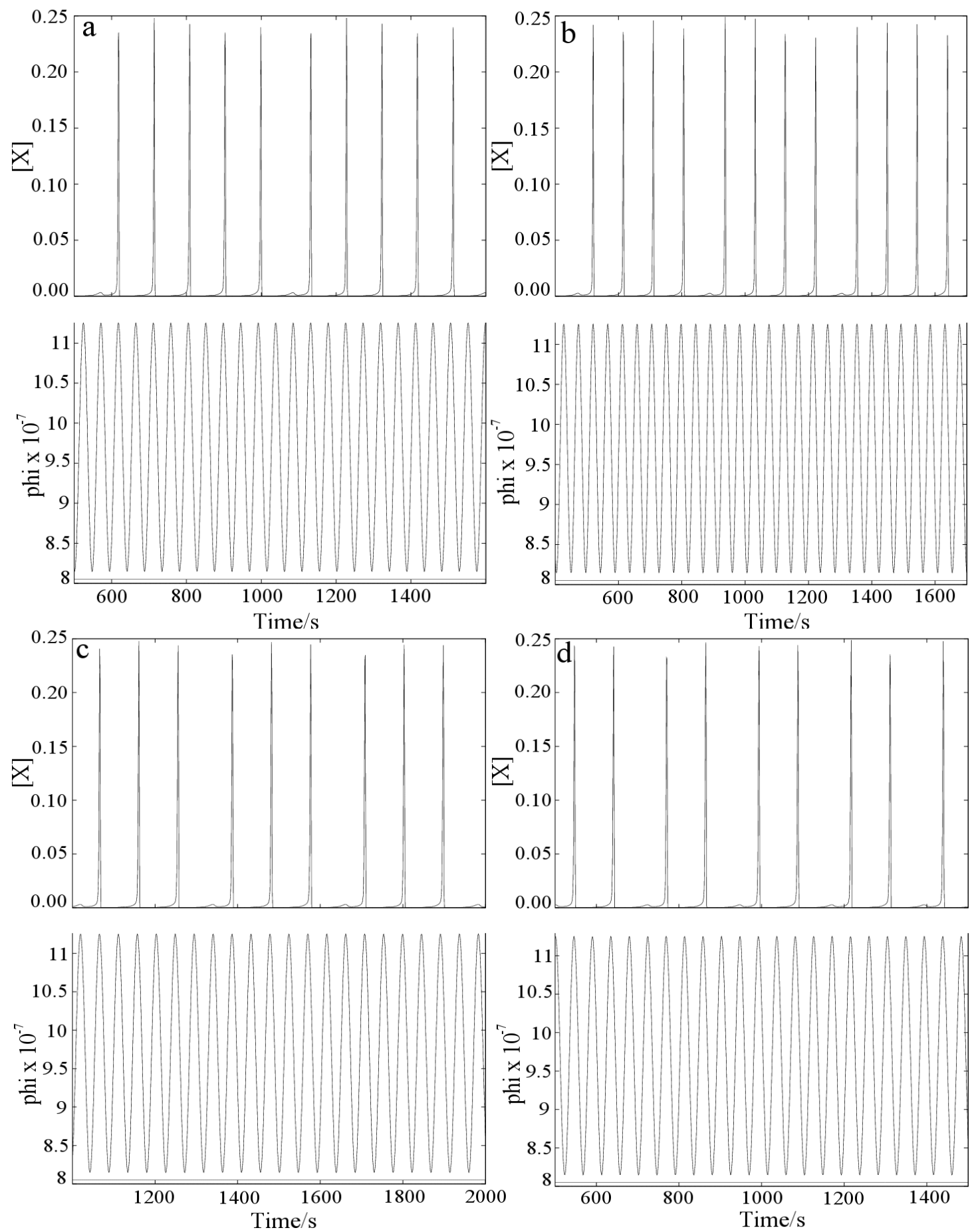

Figure 7.11. Complex simulated phase locking patterns for $\alpha=0.16$. As in Figure 7.10, the upper trace shows the time series of variable $x$, and the lower trace shows the time series of the sinusoidal perturbation, which clearly does not cross the threshold (bottom line). Model parameters are the same as in Figure 7.7. 5:11 mode locking for $v / v_{0}=1.07$ (a), 4:9 mode locking for $v / v_{0}=1.08$ (b), 3:7 mode locking for $v / v_{0}=1.09$ (c), and 2:5 mode locking for $v / v_{0}=1.12(\mathrm{~d})$. 

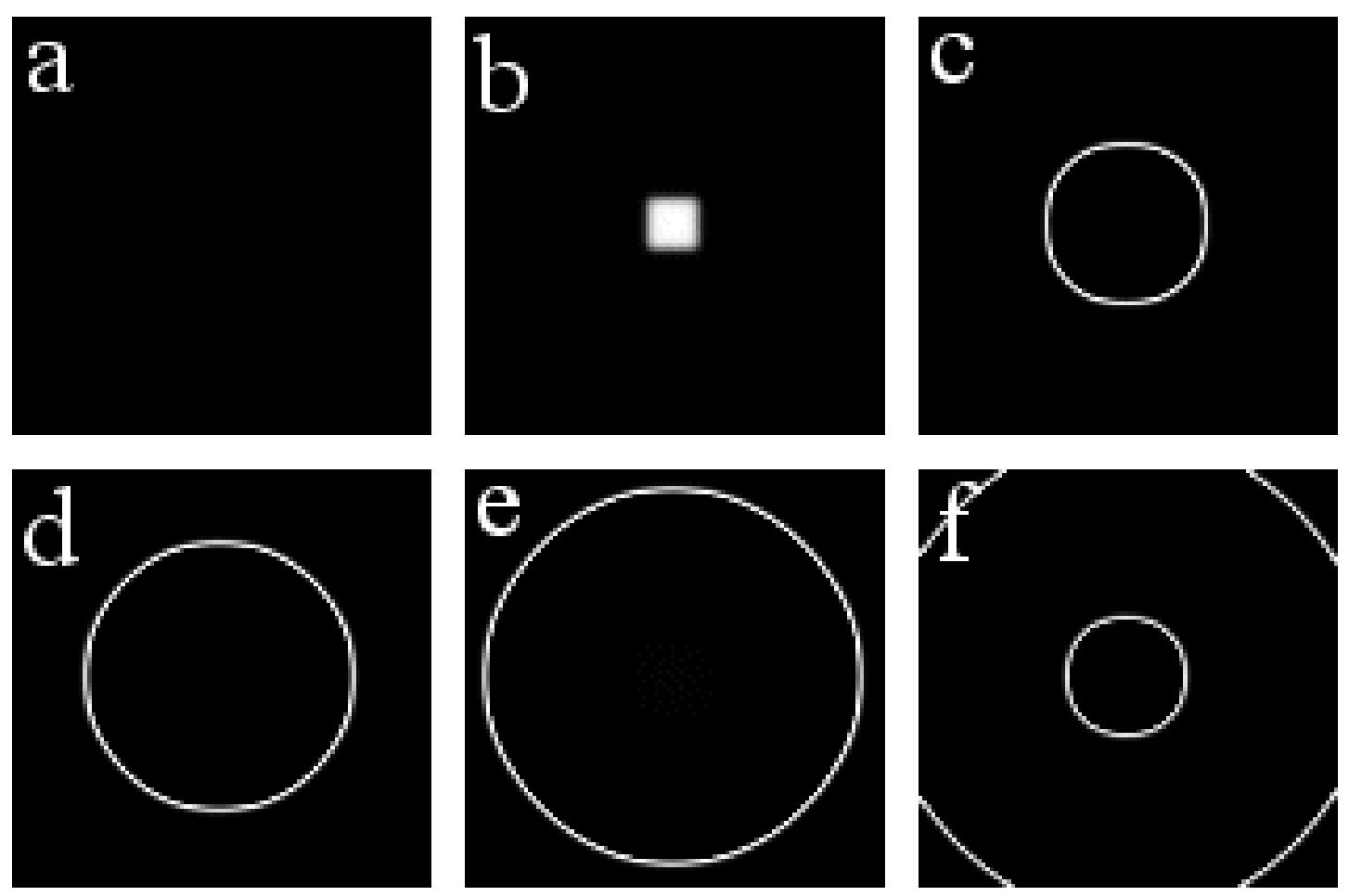

Figure 7.12: Images of an oxidation wave (white) propagating in two-dimensional excitable media (system size $=2 \mathrm{~cm}^{2}$ ) generated from the integration of the modified three-variable Oregonator model. Successive frames taken at intervals of $30 \Delta t$. Initially quiescent media, A; wave initiation due to periodic modulations in light intensity, B; oxidation wave propagates outward in the form of a traveling circular wave, $(C)-(F)$. A wave is initiated every perturbation resulting in a 1:1 resonant pattern. Model parameters are the same as in Figure 7.7. Control parameters: $v=0.50 \times v_{0}$ where $v_{0}=0.02 \mathrm{~s}^{-1}$ and $\alpha=0.16$. The simulation was carried out using a two-dimensional lattice of $100 \mathrm{x} 100$ grid points $(\Delta x=0.02, \Delta t=0.0001)$ [32]. 


\subsection{DISCUSSION AND CONCLUSION}

Our experiments and calculations show that one can initiate waves in an excitable system via small, periodic modulations in light intensity, with the number of waves initiated per number of forcing cycles giving rise to resonance patterns typical of those obtained for periodically forced systems $[32,95,96]$. Initiation of waves in an excitable media was qualitatively reproduced by numerical simulations using the modified Oregonator model. Numerical simulations also revealed the various complex resonance patterns generated in the system, which were not found in experiments. Once again we note that excitation in our case was not achieved by the traditional method of parametrically crossing the bifurcation point of the independent system, but rather by small periodic modulations in light intensity.

This work is an extension of work carried out by Parmananda [32] et al., which focused on the temporal behavior of Eqs. 7.6 without regarding the spatial behavior of the extended system. We have further extended this work by carrying out experimental investigations with the $\mathrm{BZ}$ reaction medium. 


\section{References}

1. J. Ross, S. C. Muller and C. Vidal, "Chemical waves," Science 240, 460-465 (1988).

2. A. N. Zaikin and A. M. Zhabotinskii, "Concentration wave propagation in twodimensional liquid-phase self-oscillating system," Nature 225, 535-537 (1970).

3. R. Kapral and K. Showalter, Chemical Waves and Patterns, (Kluwer Academic Publishers, Dordrecht, 1995).

4. I. R. Epstein and K. Showalter, "Nonlinear chemical dynamics: Oscillations, patterns, and chaos," J. Phys. Chem. 100, 13132-13147 (1996).

5. A. Hanna, A. Saul and K. Showalter, "Detailed studies of propagating fronts in the iodate oxidation of arsenous acid," J. Am. Chem. Soc. 104, 3838-3844 (1982).

6. T. A. Gribschaw, K. Showalter, D. L. Banville and I. R. Epstein, "Chemical waves in the acidic iodate oxidation of arsenite," J. Phys. Chem. 85, 2152-2155 (1981).

7. P. De Kepper, I. R. Epstein, K. Kustin and M. Orban, "Systematic design of chemical oscillators. Part 8. Batch oscillations and spatial wave patterns in chlorite oscillating systems," J. Phys. Chem. 86, 170-171 (1982).

8. C. Vidal and P. Hanusse, "Nonequilibrium behavior in isothermal liquid chemical systems," Int. Rev. Phys. Chem. 5, 1-55 (1986).

9. P. M. Wood and J. Ross, "A quantitative study of chemical waves in the Belousov-Zhabotinsky reaction," J. Chem. Phys. 82, 1924-1936 (1985).

10. Z. Noszticzius, W. Horsthemke, W. D. McCormick, H. L. Swinney and W. Y. Tam, "Sustained chemical waves in an annular gel reactor: A chemical pinwheel," Nature 329, 619-620 (1987).

11. S. C. Mueller, T. Plesser, A. Boiteux and B. Hess, "Pattern formation and Marangoni convection during oscillating glycolysis," J. Biosci. 40C, 588-591 (1985).

12. P. Jung, A. Cornell-Bell, F. Moss, S. Kadar, J. Wang and K. Showalter, "Noise sustained waves in sub-excitable media: From chemical waves to brain waves," Chaos 8, 567-575 (1998).

13. A. T. Winfree, "Electrical turbulence in three-dimensional heart muscle," Science 266, 1003-1006 (1994).

14. J. D. Murray, Mathematical Biology, (Springer-Verlag, Berlin, 1989). 
15. P. Jung, A. Cornell-Bell, K. S. Madden and F. Moss, "Noise-induced spiral waves in astrocyte syncytia show evidence of self-organized criticality," J. Neurophysiol. 79, 1098-1101 (1998).

16. J. Beaumont, N. Davidenko, J. M. Davidenko and J. Jalife, "Spiral waves in twodimensional models of ventricular muscle: Formation of a stationary core," Biophys. J. 75, 1-14 (1998).

17. M. A. Allessie, F. Bonke, I and F. J. Schopman, "Circus movement in rabbit atrial muscle as a mechanism of tachycardia. III. The "leading circle" concept: a new model of circus movement in cardiac tissue without the involvement of an anatomical obstacle," Circ. Res. 41, 9-18 (1977).

18. A. T. Winfree, "Sudden cardia death: A problem in topology," Sci. Am. 248, 144152 (1983).

19. J. M. Davidenko and A. M. Pertsov, "Understanding ventricular fibrillation by quantifying the complexity of activation patterns," J. Cardiovasc. Electrophysiol. 11, 1372-1374 (2000).

20. L. Wolpert, "Positional information and the spatial pattern of cellular differentiation," J. Theor. Biol. 25, 1-47 (1969).

21. A. Gierer and H. Meinhardt, "A theory of biological pattern formation," Kybernetik 12, 30-39 (1972).

22. L. R. Johnson, Physiology of the Gastrointestinal Tract, (Raven Press, New York, 1981).

23. A. L. Hodgkin and A. F. Huxley, "A quantitative description of membrane current and its application to conduction and excitation in nerve," J. Physiol. 117, 500544 (1952).

24. A. Babloyantz, Molecules, Dynamics, and Life: An Introduction To SelfOrganization of Matter, (Wiley, New York, 1986).

25. A. M. Turing, "Chemical basis of morphogenesis," Trans. Roy. Soc. B237, 37-72 (1952).

26. A. T. Winfree, "Rotating chemical reactions," Sci. Am. 230, 82-95 (1974).

27. E. Mori and J. Ross, "Origin of spontaneous wave generation in excitable chemical systems," J. Phys. Chem. 96, 8053-8060 (1992).

28. K. Showalter and R. M. Noyes, "Oscillations in chemical systems. 15. Deliberate generation of trigger waves of chemical reactivity,". J. Am. Chem. Soc. 98, 37303731 (1976). 
29. K. Showalter, R. M. Noyes and H. Turner, "Detailed studies of trigger wave initiation and detection," J. Am. Chem. Soc. 101, 7463-7469 (1979).

30. J. M. Davidenko, "Spiral wave activity: a possible common mechanism for polymorphic and monomorphic ventricular tachycardias," J. Cardiovasc. Electrophysiol. 4, 730-746 (1993).

31. Y. Nagai, H. Gonzalez, A. Shrier and L. Glass, "Paroxysmal starting and stopping of circulating waves in excitable media," Phys. Rev. Lett. 84, 4248-4251 (2000).

32. P. Parmananda, H. Mahara, T. Amemiya and T. Yamaguchi, "Resonance induced pacemakers: A new class of organizing centers for wave propagation in excitable media," Phys. Rev. Lett. 87, 238302/1-238302/4 (2001).

33. D. Mackenzie, "Mathematics in biology, News: Making sense of a heart gone wild," Science 303, 786-787 (2004).

34. A. T. Winfree, "Electrical instability in cardiac muscle: Phase singularities and rotors," J. Theor. Biol. 138, 353-405 (1989).

35. A. T. Winfree, "Spiral waves of chemical activity," Science 175, 634-636 (1972).

36. A. Toth, V. Gaspar and K. Showalter, "Signal transmission in chemical systems: Propagation of chemical waves through capillary tubes," J. Phys. Chem. 98, 522531 (1994).

37. V. A. Vavilin, A. M. Zhabotinskii and A. N. Zaikin, "Effect of ultraviolet radiation on the autovibrational oxidation of malonic acid derivatives," Russ. J. Phys. Chem. 42, 3091-3094 (1968).

38. M. Jinguji, M. Ishihara and T. Nakazawa, "Photoinduced formation of spatial patterns in the Belousov-Zhabotinsky reaction," J. Phys. Chem. 94, 1226-1229 (1990).

39. A. Pagola, J. Ross and C. Vidal, "Measurement of dispersion relation of chemical waves in an oscillatory reacting medium," J. Phys. Chem. 92, 163-166 (1988).

40. K. Suzuki, T. Yoshinobu and H. Iwasaki, "Induction of chemical waves by mechanical stimulation in elastic Belousov-Zhabotinskii media," Chem. Phys. Lett. 349, 437-441 (2001).

41. E. Mori, X. Chu and J. Ross, "Chemical front propagation: Initiation and relative stability," in Chemical Waves and Patterns, edited by R. Kapral and K. Showalter, (Kluwer Academic Publishers, Dordrecht, 1995) p. 419-446.

42. H. Sevcikova and M. Marek, "Wave patterns in an excitable reaction-diffusion system," Physica D 49, 114-124 (1991). 
43. D. R. Chialvo and J. Jalife, "Non-linear dynamics of cardiac excitation and impulse propagation," Nature 330, 749-752 (1987).

44. A. T. Winfree, "Biological rhythms and the behavior of populations of coupled oscillators," J. Theor. Biol. 16, 15-42 (1967).

45. L. Kuhnert, K. I. Agladze and V. I. Krinskii, "Image processing using lightsensitive chemical waves," Nature 337, 244-247 (1989).

46. A. M. Zhabotinsky, Oscillatory Processes in Biological and Chemical Systems, (Science Publishers, Moscow, 1967) p. 252.

47. R. J. Field, E. Koros and R. M. Noyes, "Oscillations in chemical systems. II. Thorough analysis of temporal oscillation in the bromate-cerium-malonic acid system," J. Am. Chem. Soc. 94, 8649-8664 (1972).

48. R. M. Noyes, R. Field and E. Koros, "Oscillations in chemical systems. I. Detailed mechanism in a system showing temporal oscillations," J. Am. Chem. Soc. 94, 1394-1395 (1972).

49. R. M. Noyes and R. J. Field, "Oscillations in chemical systems. 19. Mechanisms of chemical oscillators: experimental examples," Accounts Chem. Res. 10, 273280 (1977).

50. E. Koros, L. Ladanyi, V. Friedrich, Z. Nagy and A. Kis, "Tris(dipyridine)ruthenium(2+) ion-bromate-malonic acid oscillating system," React. Kinet. Catal. Lett. 1, 455-460 (1974).

51. T. C. Bruice and G. J. Kasperek, "Oscillating reaction. Reaction of potassium bromate, ceric sulfate, and a dicarboxylic acid," Inorg. Chem. 10, 382-386 (1971).

52. S. S. Jacobs and I. R. Epstein, "Effects of chloride ion on oscillations in the bromate-cerium-malonic acid system," J. Am. Chem. Soc. 98, 1721-1724 (1976).

53. H. Busse and B. Hess, "Information transmission in a diffusion-coupled oscillatory chemical system," Nature 244, 203-205 (1973).

54. V. Gaspar, G. Bazsa and M. T. Beck, "The influence of visible light on the Belousov-Zhabotinsky oscillating reactions applying different catalysts," Z. Phys. Chem. (Leipzig) 264, 43-48 (1983).

55. L. Kuhnert, "A new optical photochemical memory device in a light-sensitive chemical active medium," Nature 319, 393-394 (1986).

56. K. Kalyanasundaram, "Photophysics, photochemistry and solar energy conversion with tris(bipyridyl)ruthenium(II) and its analogs," Coordin. Chem. Rev. 46, 159244 (1982). 
57. S. Kadar, T. Amemiya and K. Showalter, "Reaction mechanism for light sensitivity of the $\mathrm{Ru}(\mathrm{bpy})_{3}{ }^{2+}$-catalyzed Belousov-Zhabotinsky reaction," J. Phys. Chem. A 101, 8200-8206 (1997).

58. J. J. Tyson and J. P. Keener, "Singular pertubation theory of traveling waves in excitable media (A review)," Physica D 32, 327-367 (1988).

59. A. S. Mikhailov and V. I Krinskii, "Reverberator in an active medium. Analytical results," Biofizika 27, 875-879 (1982).

60. J. P. Keener and J. J. Tyson, "Spiral waves in the Belousov-Zhabotinskii reaction," Physica D 21D, 307-324 (1986).

61. R. J. Field and Burger Maria, Oscillations and Traveling Waves in Chemical Systems, (John Wiley and Sons, New York, 1985).

62. P. Parmananda, "Generalized synchronization of spatiotemporal chemical chaos," Phys. Rev. E 56, 1595-1598 (1997).

63. P. Jung and P. Haenggi, "Resonantly driven brownian motion: Basic concepts and exact results," Phys. Rev. A 41, 2977-2988 (1990).

64. L. Gammaitoni, P. Hanggi, P. Jung and F. Marchesoni, "Stochastic resonance," Rev. Mod. Phys. 70, 223-287 (1998).

65. A. R. Bulsara, S. B. lowen and C. D. Rees, "Reply to 'coherent stochastic resonance in the presence of a field'," Phys. Rev. E 52, 5712-5713 (1995).

66. B. Shulgin, A. Neiman and V. Anishchenko, "Mean switching frequency locking in stochastic bistable systems driven by a periodic force," Phys. Rev. Lett. 75, 4157-4160 (1995).

67. V. Petrov, Q. Ouyang and H. L. Swinney, "Resonant pattern formation in a chemical system," Nature 388, 655-657 (1997).

68. S. Kadar, J. Wang and K. Showalter, "Noise-supported travelling waves in subexcitable media," Nature 391, 770-772 (1998).

69. S. Grill, V. S. Zykov and S. C. Mueller, "Spiral wave dynamics under pulsatory modulation of excitability," J. Phys. Chem. 100, 19082-19088 (1996).

70. V. S. Zykov, S. Grill and S. C. Mueller, "Order and chaos in excitable media under external forcing," Proc. Exp. Chaos Conf. 3, 185-199 (1996).

71. O. Steinbock and S. C. Mueller, "Chemical spiral rotation is controlled by lightinduced artificial cores," Physica A 188, 61-67 (1992). 
72. M. McGuinness, Y. Hong, D. Galletly and P. Larsen, "Arnold tongues in human cardiorespiratory systems," Chaos 14, 1-6 (2004).

73. A. L. Lin, M. Bertram, K. Martinez, H. L. Swinney, A. Ardelea and G. F. Carey, "Resonant phase patterns in a reaction-diffusion system," Phys. Rev. Lett. 84, 4240-4243 (2000).

74. V. Paar and N. Pavin, "Intermingled fractal Arnold tongues," Phys. Rev. E 57, 1544-1549 (1998).

75. K. Yoshino, T. Nomura, K. Pakdaman and S. Sato, "Synthetic analysis of periodically stimulated excitable and oscillatory membrane models," Phys. Rev. E 59, 956-969 (1999).

76. S. Coombes and P. C. Bressloff, "Mode locking and Arnold tongues in integrateand-fire neural oscillators," Phys. Rev. E 60, 2086-2096 (1999).

77. L. Glass, "Cardiac arrhythmias and circle maps - A classical problem," Chaos 1, 13-19 (1991).

78. V. I. Arnold, "Cardiac arrhythmias and circle mappings," Chaos 1, 20-24 (1991).

79. J. Belair, "Periodic pulsatile stimulation of a nonlinear oscillator," J. Math. Biol. 24, 217-232 (1986).

80. M. H. Jensen, P. Bak and T. Bohr, "Complete devil's staircase, fractal dimension, and universality of mode-locking structure in the circle map," Phys. Rev. Lett. 50, 1637-1639 (1983).

81. M. H. Jensen, P. Bak and T. Bohr, "Transition to chaos by interaction of resonances in dissipative systems. I. Circle maps," Phys. Rev. A 30, 1960-1069 (1984).

82. A. Pikovsky, M. Rosenblum, and J. Kurths, Synchronization: A Universal Concept in Nonlinear Science, (Cambridge University Press, Cambridge 2003).

83. R. J. Field and R. M. Noyes, "Oscillations in chemical systems. IV. Limit cycle behavior in a model of a real chemical reaction," J. Chem. Phys. 60, 1877-1884 (1974).

84. T. Amemiya, T. Ohmori, M. Nakaiwa and T. Yamaguchi, "Two-parameter stochastic resonance in a model of the photosensitive Belousov-Zhabotinsky reaction in a flow system," J. Phys. Chem. A 102, 4537-4542 (1998).

85. A. Juris, V. Balzani, F. Barigelletti, S. Campagna, P. Belser and A. Von Zelewsky, "Ruthenium(II) polypyridine complexes: Photophysics, photochemistry, electrochemistry, and chemiluminescence," Coordin. Chem. Rev. 84, 85-277 (1988). 
86. M. K. R. Reddy, Z. Nagy-Ungvarai and S. C. Mueller, "Effect of visible light on wave propagation in the ruthenium-catalyzed Belousov-Zhabotinsky reaction," J. Phys. Chem. 98, 12255-12259 (1994).

87. T. Yamaguchi, L. Kuhnert, Z. Nagy-Ungvarai, S. C. Mueller and B. Hess, "Gel systems for the Belousov-Zhabotinsky reaction," J. Phys. Chem. 95, 5831-5837 (1991).

88. A. B. Katok and B. Hasselblatt, Introduction to the Modern Theory of Dynamical Systems, (Cambridge University Press, Cambridge, 1995).

89. J. J. Tyson and P. C. Fife, "Target patterns in a realistic model of the BelousovZhabotinsky reaction," J. Chem. Phys. 73, 2224-2237 (1980).

90. H. J. Krug, L. Pohlmann and L. Kuhnert, "Analysis of the modified complete Oregonator accounting for oxygen sensitivity and photosensitivity of BelousovZhabotinsky systems," J. Phys. Chem. 94, 4862-4866 (1990).

91. J. J. Tyson, "Oscillations, bistability, and echo waves in models of the BelousovZhabotinsky reaction," Ann. N. Y. Acad. Sci. 316, 279-295 (1979).

92. J. Maselko and H. L. Swinney, "A Farey triangle in the Belousov-Zhabotinsky reaction," Phys. Lett. A 119, 403-406 (1987).

93. D. D. Coon, S. N. Ma and A. G. U. Perera, "Farey-fraction frequency modulation in the neuronlike output of silicon p-i-n diodes at $4.2 \mathrm{~K}$," Phys. Rev. Lett. 58, 1139-1142 (1987).

94. M. James and F. Moss, "Analog simulation of a periodically modulated laser model," J. Opt. Soc. Am. B 5, 1121-1126 (1988).

95. J. Finkeova, M. Dolnik, B. Hrudka and M. Marek, "Excitable chemical reaction systems in a continuous stirred tank reactor," J. Phys. Chem. 94, 4110-4115 (1990).

96. M. Marek, M. Dolnik, I. Schreiber and J. Finkeova, "Excitable chemical reaction systems," NATO ASI Series, Series B 244, 79-89 (1991). 


\section{CHAPTER 8}

\section{Chemical Waves in the Oscillatory Peroxidase-Oxidase Reaction}

\subsection{INTRODUCTION}

Chemical waves are comprised of concentration gradients propagating in an excitable reaction medium. They have been extensively studied both theoretically and experimentally [1,2]. Most studies have focused on biological [3-5], biochemical [6], and chemical systems $[7,1]$, with the BZ reaction being subjected to more extensive studies, as it is much easier to experimentally prepare and control as well as study computationally.

Theoretical and experimental studies have distinguished between two types of chemical waves: phase waves and trigger waves [8-10]. Phase waves, also known as pseudowaves [10,11], long waves [12], phase diffusion waves [13], or kinematic waves $[13,14]$, occur in oscillatory media due to variations of a state variable in space, giving rise to phase or frequency differences or gradients in the concentrations of certain species $[9,14]$. Phase waves do not rely on diffusion for their propagation, and are thus able to pass through impermeable barriers. One of the distinguishing characteristics of phase waves is that they propagate at high velocities without an upper velocity limit $[9,15]$.

Trigger waves or short waves [12] occur in excitable media and are dependent upon diffusion for their propagation. Two conditions must be met for the onset of trigger waves [9,14]: First, the system should be excitable, i.e., perturbations exceeding a certain threshold of concentration for a given variable should trigger a large excursion in phase space. An oscillatory medium may also be excitable; hence, trigger waves can occur in 
oscillatory or non-oscillatory excitable media. Second, those variables capable of giving rise to a large excursion in phase space should have a steep concentration gradient in space. Trigger waves can be distinguished from phase waves in that they travel at constant velocity (smaller velocity when compared to phase waves), have sharp wave fronts, and are unable to cross impenetrable barriers $[9,13]$.

In the previous chapter, we reported on the successful initiation of resonance induced waves in an excitable BZ medium, and these waves were modeled by solving the reaction-diffusion equations of the modified Oregonator model. In this chapter, we report on the initiation of chemical waves in an oscillatory biochemical system, the peroxidaseoxidase reaction. Trigger waves are initiated in the oscillatory system via localized perturbations in the concentration of one of the variables using the extended BFSO model [16-18]. In this chapter we will also review the experimental and theoretical studies of the peroxidase-oxidase reaction that have been previously carried out.

\subsection{THE PEROXIDASE-OXIDASE REACTION}

The peroxidase-oxidase reaction was first reported by Yokota and Yamazaki in $1965[19,20]$. They observed damped oscillations in the oxidation of NADH catalyzed by horseradish peroxidase, HRP, with oxygen as the oxidizing agent. This reaction has been extensively studied over the years both theoretically and experimentally. Most studies have been conducted using peroxidase enzyme from horseradish roots, although peroxidases from other compounds, e.g. milk [21-23], soybeans [22,23], or the fungus Coprinus cinereus $[22,23]$, have been shown also to catalyze the oscillatory reaction. 
Peroxidases are enzymes which catalyze the oxidation of compounds that are hydrogen donors. In most instances hydrogen peroxide, $\mathrm{H}_{2} \mathrm{O}_{2}$, is the oxidizing agent, and the reaction is given by the following stoichiometry:

$$
2 \mathrm{YH}+\mathrm{H}_{2} \mathrm{O}_{2} \rightarrow 2 \mathrm{Y}^{\bullet}+2 \mathrm{H}_{2} \mathrm{O}
$$

where $\mathrm{YH}$ is the hydrogen donor. For some substances, e.g. reduced nicotinamide adenine dinucleotide (NADH), indole-3-acetic acid, dihydroxyfumaric acid, or triose reductone, oxygen $\left(\mathrm{O}_{2}\right)$ can act as the oxidizing agent in place of $\mathrm{H}_{2} \mathrm{O}_{2}$, and equation (8.1) becomes [24]:

$$
2 \mathrm{YH}+\mathrm{O}_{2}+2 \mathrm{H}^{+} \rightarrow 2 \mathrm{Y}^{+}+2 \mathrm{H}_{2} \mathrm{O}
$$

Most experimental and theoretical studies have been carried out using NADH as the hydrogen donor, and equation (8.2) is replaced by the following stoichiometry:

$$
2 \mathrm{NADH}+\mathrm{O}_{2}+2 \mathrm{H}^{+} \rightarrow 2 \mathrm{NAD}^{+}+2 \mathrm{H}_{2} \mathrm{O}
$$

This reaction, when studied in the presence of the modifiers methylene blue (MB) and 2,4-dichlorophenol (DCP), exhibits a variety of nonlinear phenomena under semibatch or CSTR conditions. The reported behaviors include damped oscillations [19], simple [21] and mixed mode oscillations [25-27], bursting oscillations [17,28], quasiperiodicity [26,29], period adding and period doubling route to chaos [30-32]. 
Theoretical studies of reaction (8.3) have been hampered by the complicated mechanism of the reaction, which includes multiple enzyme states and the generation of intermediates, such as $\mathrm{H}_{2} \mathrm{O}_{2}$, NAD', and superoxide anion $\left(\mathrm{O}_{2}{ }^{-}\right)$. There are five enzyme states of HRP, differing in oxidation state, which are involved in the PO reaction [33,34]: ferrous peroxidase $\left(\mathrm{Per}^{2+}\right)$, ferriperoxidase $\left(\mathrm{Per}^{3+}\right)$ or the native form, compound I (CoI or $\mathrm{Per}^{5+}$ ), compound II (CoII or $\mathrm{Per}^{4+}$ ), and compound III (CoIII or $\mathrm{Per}^{6+}$ ). Other states have also been reported, but these are not known to partake in the PO reaction.

The PO reaction involves two feedback loops (oxidation-reduction loops) by which reactants are transformed into products. The first loop, the peroxidase cycle, involves the initial oxidation of the native form by $\mathrm{H}_{2} \mathrm{O}_{2}$.

$$
\begin{aligned}
& \mathrm{Per}^{3+}+\mathrm{H}_{2} \mathrm{O}_{2} \rightarrow \mathrm{CoI} \\
& \mathrm{CoI}+\mathrm{YH} \rightarrow \mathrm{CoII}+\mathrm{YH}^{\bullet} \\
& \mathrm{CoII}+\mathrm{YH} \rightarrow \mathrm{Per}^{3+}+\mathrm{YH}^{\bullet}
\end{aligned}
$$

The second loop, the oxidase cycle, involves the reduction of CoIII to CoI.

$$
\begin{aligned}
& \mathrm{Per}^{3+}+\mathrm{O}_{2}^{-} \rightarrow \mathrm{CoIII} \\
& \mathrm{CoIII}+\mathrm{Y}^{\cdot} \rightarrow \mathrm{CoI}+\mathrm{Y}^{+} \\
& \mathrm{CoI}+\mathrm{YH} \rightarrow \mathrm{CoII}+\mathrm{YH}^{\bullet} \\
& \mathrm{CoII}+\mathrm{YH} \rightarrow \mathrm{Per}^{3+}+\mathrm{YH}^{\bullet} \\
& \mathrm{Per}^{3+}+\mathrm{YH}^{\cdot} \rightarrow \mathrm{Per}^{2+}+\mathrm{Y}^{+} \\
& \mathrm{Per}^{2+}+\mathrm{O}_{2} \rightarrow \mathrm{CoIII}
\end{aligned}
$$


Of special importance in the PO reaction mechanism are the NAD ${ }^{\circ}$ radical and CoIII species.

\subsubsection{Experimental Studies}

\subsubsection{Early Work}

Damped oscillations in the oxidation of NADH were first reported in 1965 [19], when the reaction was carried out in an semi-open system, where oxygen was continuously bubbled into the system. Compound III was thought to have an important role in the observed behavior, while the reaction was thought to be inhibited by oxygen. Later work by Degn [24], demonstrated that one could also observe the same phenomena when NADH was substituted by dihydroxyfumaric acid, triose reductone, and indole-3acetic acid.

Sustained oscillations were later observed in a slightly modified system where the hydrogen donor, NADPH, was regenerated using a glucose-6-phospate and glucose-6phospate dehydrogenase combination in the presence of $\mathrm{MB}$ and $\mathrm{DCP}$ with either lactoperoxidase [21] or HRP [35]. Olsen and Degn [36,37] later demonstrated that glucose-6-phophate dehydrogenase was not a prerequisite for sustained oscillations, as they observed the same phenomena when NADH was constantly infused into the system. The presence of the modifiers, MB and DCP, was critical, however, for sustained oscillations. 


\subsubsection{Observation of Chaos}

Olsen and Degn [37] observed that the waveform of oscillations changed from periodic to aperiodic as the initial enzyme concentrations were varied. Initial enzyme concentrations of $0.9 \mu \mathrm{M}$ gave simple periodic oscillations, and bursting oscillations were observed for concentrations below $0.5 \mu \mathrm{M}$. Irregular and aperiodic oscillations were observed for initial enzyme concentrations of $0.5 \mu \mathrm{M}$. An analysis of these results using next-amplitude maps and the theorem of $\mathrm{Li}$ and Yorke [38] were carried out to prove that the aperiodic oscillations were chaotic, although no route to chaos was determined. Chaos was reported in the BZ reaction by Schmitz et al. [39] only after it had been observed in the PO reaction. The PO reaction is the only single-enzyme reaction known to exhibit chaotic phenomena without the imposition of periodic forcing.

Work by Geest et al. [31] in 1992 demonstrated that the periodicity of the oscillations could be changed by altering the concentrations of DCP. They observed a period-doubling route to chaos as concentrations of DCP were increased. Later work by Hauser et al. $[25,30]$ confirmed the existence of this period-doubling route to chaos at $\mathrm{pH}$ 5.1 when the concentrations of NADH was increased. Increasing NADH concentrations at $\mathrm{pH}$ 6.3, however, resulted in a period adding route to chaos when all other experimental conditions were kept constant. The transformation from a period-adding route to a period-doubling route occurred at a $\mathrm{pH}$ of about 5.4.

\subsubsection{The Role of Methylene Blue}

The PO reaction is traditionally studied in the presence of modifiers, MB and DCP, introducing nonphysiological species into the system. Simple oscillations have 
been observed in the absence of the modifiers, although their presence was a prerequisite for one to observe complex dynamics. Before studies by Hauser et al. [40], MB was believed to be an inhibitor of enzyme activity [21,36].

Studies $[40,41,42]$ have revealed that damped oscillations can be observed in the absence of MB; the enzyme, however, was inactivated or destroyed in such cases. Addition of moderate amounts of MB gave sustained oscillations and prevented the irreversible inactivation of HRP. MB also influenced the amount or turnover of ferrous peroxidase in the system. The authors concluded that MB reacted either directly with ferrous peroxidase or with $\mathrm{NAD}^{\circ}$ radical, hence, indirectly affecting ferrous peroxidase production. Theoretical models of the PO reaction have accounted for MB in the mechanism by including its concentration into the rate constant of the following reaction:

$$
\mathrm{NADH}+\mathrm{H}^{+}+\mathrm{O}_{2} \rightarrow \mathrm{NAD}^{+}+\mathrm{H}_{2} \mathrm{O}_{2}
$$

\subsubsection{The Role of 2,4 Dichlorophenol}

Use of the nonphysiological DCP as a modifier in the PO reaction questions the physiological applications of the reaction. Studies have shown that DCP can be substituted by other phenolic or aromatic amines, which also leads to simple and complex dynamics [43-45]. No oscillations or only damped oscillations were observed in the absence of phenolic amines [43], confirming that they are essential for sustained oscillations. Addition of moderate amounts of phenolic cofactors yielded different results depending on the substituents. Compounds such as 2-hydroxycinnamic acid, phenol, and p-cresol, completely inhibited the PO reaction while 4-hydroxybenzaldehyde or 4- 
hydroxycinnamic acid caused the PO reaction to proceed in a monotonic way. Sustainable periodic oscillations were observed with 2-chlorophenol and 3-chlorophenol, while complex dynamics were observed with 2,4-dichlorophenol, 7-hydroxycoumarin, and 4-chlorophenol. For an aromatic compound to induce complex dynamics in the PO reaction, an aromatic hydroxyl group or an aromatic amino group was required $[43,45]$. Possession of only an aromatic hydroxyl group, however, did not induce complex dynamics.

Phenolic compounds aid in electron transfer in the PO reaction and are not used up during the reaction. Whether the addition of a phenolic compound resulted in complex dynamics depended on the half-wave potential for the following reaction:

$$
\mathrm{PhOH} \rightarrow \mathrm{PhO}^{\bullet}+\mathrm{H}^{+}+\mathrm{e}^{-}
$$

\subsubsection{Bistability}

Experiments by Degn [46] and later by Degn et al. [47] revealed the occurrence of bistability in the PO reaction. Two stable steady states were found for the same experimental conditions, i.e. oxygen partial pressure. The system could be reversibly switched from one state to another by perturbing the oxygen concentration. The bistability phenomenon was attributed to enzyme inhibition by oxygen.

Work by Auda et al. [48,28] has shown that the system can also be bistable between an oscillatory state and a steady state. Reversible transitions from one state to another were achieved by momentarily stopping oxygen supply to the reactor (steady 
state $\rightarrow$ oscillations) or by perturbing the hydrogen peroxide concentration (oscillations $\rightarrow$ steady state).

\subsubsection{Theoretical Studies}

\subsubsection{Introduction}

Extensive studies have been carried out on theoretically modeling the PO reaction, beginning with the work by Olsen and Degn [36]. A number of models have been proposed, which were developed as an understanding of the reaction grew. The models can be divided into two categories: simple models, e.g. the 1983 Olsen model, and detailed models, e.g. BFSO model, both of which will be discussed in this section.

The detailed models attempt to include all of the essential reactions into the mechanism, and the number of number of reactions increased as an understanding of the PO reaction developed. Both the simple and detailed models are able to produce results similar to those obtained in experiments, although the simple models had been superior before the development of the BFSO model [16]. This paradox was attributed to inaccuracies in the rate constants used in the detailed models. The detailed models have a number reactions in common, specifically reactions involving four of the five enzyme species [34]. Models that incorporated the fifth species, $\mathrm{Per}^{2+}$, were able to show complex

dynamics, while only simple oscillations were observed with models in which $\mathrm{Per}^{2+}$ was excluded in the mechanism [49]. Another important species is the NAD• radical, which is believed to be the autocatalyst in the PO reaction. 


\subsubsection{Simple Models}

\subsection{The Four-Variable Model}

Theoretical modeling of the PO reaction began with the work of Degn, Olsen, and Perram [47], who developed a four-variable model for the oxidation of NADH catalyzed by HRP. The four variables represent the reactants, molecular oxygen (A) and NADH (B), and intermediates, $\mathrm{NAD}^{\circ}(\mathrm{X})$ and CoIII $(\mathrm{Y})$. The native form of the enzyme is included in the rate constants for the reactions (A1) and (A3). The modifiers MB and DCP are not explicitly accounted for in this model. The model is given by the following equations:

$$
\begin{gathered}
\mathrm{A}+\mathrm{B}+\mathrm{X} \rightarrow 2 \mathrm{X} \\
2 \mathrm{X} \rightarrow 2 \mathrm{Y} \\
\mathrm{A}+\mathrm{B}+\mathrm{Y} \rightarrow 2 \mathrm{X} \\
\mathrm{X} \rightarrow \mathrm{P} \\
\mathrm{Y} \rightarrow \mathrm{Q} \\
\mathrm{X}_{0} \rightarrow \mathrm{X} \\
\mathrm{A}_{0} \rightleftharpoons \mathrm{A} \\
\mathrm{B}_{0} \rightarrow \mathrm{B}
\end{gathered}
$$

where (A1) is a branching reaction and reactions (A4) and (A5) are termination reactions. Reaction (A6) is the initiation reaction involving the formation of the free radicals NAD', and reactions (A7) and (A8) model the inflow of oxygen and NADH into the reactor. This model was able to yield simulations in good agreement with experimental data, with one drawback. The model did not reproduce complex dynamics observed in experiments. 
Later work by Larter et al. [50,51], however, showed that the model was able to generate complex dynamics for a small parameter region.

In 1983, Olsen [52] proposed a slightly modified model that is similar to the DOP model except for the first and third reactions, which are given by the following:

$$
\begin{array}{r}
\mathrm{B}+\mathrm{X} \rightarrow 2 \mathrm{X} \\
\mathrm{A}+\mathrm{B}+\mathrm{Y} \rightarrow 3 \mathrm{X}
\end{array}
$$

This model is able to reproduce the experimental results both qualitatively and quantitatively $[50,51,53]$. The 1983 Olsen model produces complex dynamics for a wider range of variables when compared to the DOP model.

\subsection{The Dunford Model}

In 1991 Alexandre and Dunford [54] proposed a model for the PO reaction as they argued that the 1983 Olsen model was not compatible with experiments as it did not include oxygen in the first loop. They argued that in the Olsen and DOP model CoIII was used up instead of being formed when the concentration of oxygen was high as one would expect from experimental data. The reactions of the model are:

$$
\begin{aligned}
\mathrm{P}+\mathrm{A}+\mathrm{X} & \rightarrow \mathrm{Y}+2 \mathrm{X} \\
\mathrm{Y}+\mathrm{X} & \rightarrow \mathrm{P}+2 \mathrm{X} \\
\mathrm{B} & \rightarrow \mathrm{X} \\
\mathrm{X} & \rightarrow \text { Products } \\
\mathrm{A}_{0} & \rightleftharpoons \mathrm{A}
\end{aligned}
$$


The variables are (A) oxygen, (B) NADH, which is assumed to be constant, (X) is the NAD ${ }^{\circ}$ radical, $(\mathrm{Y})$ is CoIII, and $(\mathrm{P})$ is the native state of the enzyme $\left(\mathrm{Per}^{3+}\right)$. Reactions (B1) and (B2) comprise the autocatalytic cycle. Reaction (B3) is the initiation reaction in which $\mathrm{NAD}^{\circ}$ radicals are formed. Reaction (B5) models the inflow of oxygen into the system. This model was able to simulate both damped and periodic oscillations; it failed, however, to reproduce the complex dynamics observed in the PO reaction.

\subsubsection{Detailed Models}

\subsection{The YY Model}

In 1977, Yokota and Yamazaki proposed a 12 reaction closed system model for the PO reaction called the YY model (see Table 8.1) [55]. This model simulates a closed PO system where there is no inflow of reactants or outflow of products. The model did not include the fifth enzyme species, $\mathrm{Per}^{2+}$ or a termination pathway for the NAD ${ }^{\circ}$ radicals. It was able to simulate the characteristic waveform of the reaction with the distinctive phases in the dynamics of CoIII. The model was, however, unable to reproduce the oscillatory dynamics observed in experiments [34]. This was attributed to inaccuracies in rate constants and inconsistencies in the mechanism as discussed by Aguda and Larter [48].

\subsection{The FAB Model}

The FAB model [56], like the YY model, does not include the fifth enzyme

species, $\mathrm{Per}^{2+}$, but differs from the YY model by the inclusion of a unimolecular termination pathway for the $\mathrm{NAD}^{\circ}$ radical. The $\mathrm{FAB}$ model is able to simulate oscillatory 
dynamics, although no quantitative analysis could be carried out due to the assumptions made in simplifying the model. The model, however, was unable to simulate the complex dynamics observed in experiments.

\subsection{Model A}

Aguda and Clark [57] in 1987 proposed a model based on reducing the list of PO reactions given by Yamazaki and Yokota [55] via stoichiometric network analysis. This model, like the FAB model includes a termination reaction for the NAD radical and does not include the enzyme species $\mathrm{Per}^{2+}$, but differs in that the termination reaction is now bimolecular. This model was capable of reproducing damped oscillations, bistability, and simple periodic oscillations [34,48]. No complex oscillatory dynamics or chaos was observed in the simulations with Model A.

\subsection{Model C}

To model complex dynamics in the PO reaction, Aguda and Clark [48] modified Model A to include the fifth enzyme species, $\mathrm{Per}^{2+}$, and time-dependent inflow of NADH into the system as in experiments. Numerical simulations using this model, Model C (C for chaos) [34], gave both periodic and chaotic oscillations via a period-doubling route [58]. The results were both qualitative and quantitative. It was concluded that two feedback loops were essential for one to observe complex dynamics, and this was achievable by inclusion of $\mathrm{Per}^{2+}$. 


\subsection{Urbanalator}

To propose a model as close to experiments as possible, the Urbanalator [59] was formulated using data (reactions and rate constants) that had been experimentally derived. This model included the modifier MB in its mechanism, but not the fifth enzyme species, $\mathrm{Per}^{2+}$. This model is capable of producing simple oscillation, although no complex oscillation or chaos was reported.

\subsection{BFSO}

To reproduce complex dynamics and chaos in the PO reaction, Bronnikova et al. [16] modified the Urbanalotor to include the fifth enzyme species, $\mathrm{Per}^{2+}$. These adjustments were similar to those done by Aguda and Clark in modifying Model A to Model C. Numerical simulations of the BFSO model were able to qualitatively reproduce both periodic oscillations and chaotic oscillations via a period-doubling route as observed in experiments $[31,30,60]$. The BFSO model was modified in 2001 to include (R19) to give the BFSO-14 model [17]. The extended BFSO model is capable of simulating the observed bistability and bursting oscillations at low enzyme concentrations [18]. Although some discrepancies still exist between experimental and simulated behavior, the BFSO-14 model is at present model of choice for the PO reaction as it uses experimentally realistic rate equations and rate constants. It is for this reason the BFSO14 model was chosen for simulating chemical waves in the PO reaction. 
Table 8.1 Reactions Included in Detailed Models [34].

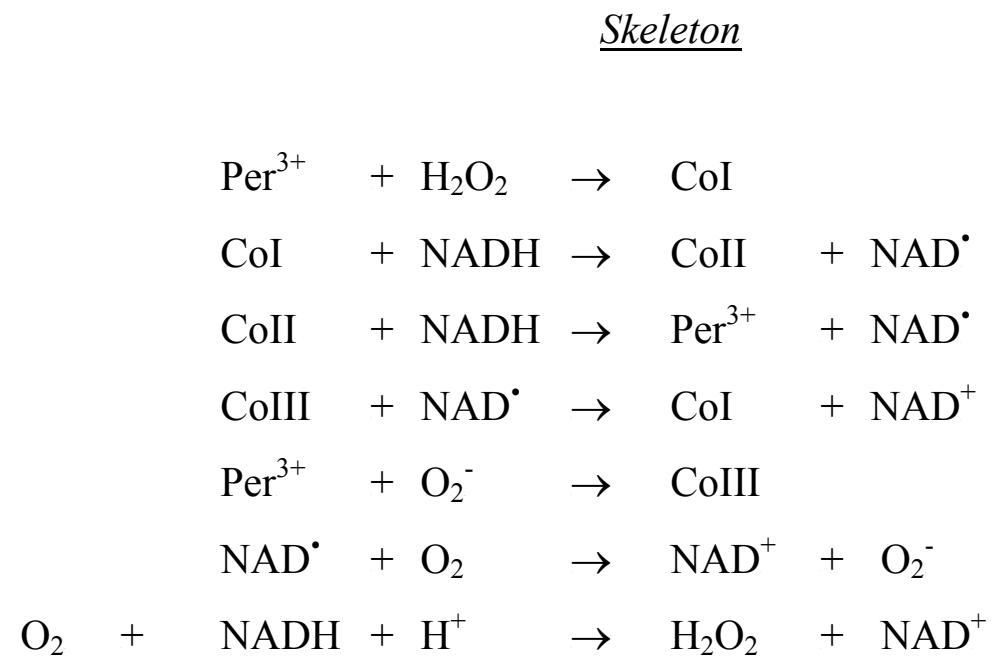

\section{$\underline{\text { Model } A}$}

$$
\begin{aligned}
2 \mathrm{NAD}^{\circ} & \rightarrow(\mathrm{NAD})_{2} \\
& \rightarrow \mathrm{O}_{2} \\
\mathrm{O}_{2} & \rightarrow
\end{aligned}
$$

\section{Model C}

$$
\begin{aligned}
& \rightarrow \quad \mathrm{NADH} \\
& \mathrm{Per}^{3+}+\mathrm{NAD}^{\cdot} \rightarrow \mathrm{Per}^{2+}+\mathrm{NAD}^{+} \\
& \mathrm{Per}^{2+}+\mathrm{O}_{2} \rightarrow \text { CoIII } \\
& 2 \mathrm{O}_{2}^{-}+2 \mathrm{H}^{+} \rightarrow \mathrm{O}_{2}+\mathrm{H}_{2} \mathrm{O}_{2} \\
& \mathrm{H}^{+}+\mathrm{H}_{2} \mathrm{O}_{2}+\mathrm{NAD}^{\circ} \rightarrow \mathrm{NAD}^{+}+\mathrm{H}_{2} \mathrm{O}+\mathrm{HO}^{\circ} \\
& \mathrm{HO}^{\circ}+\mathrm{NADH} \rightarrow \mathrm{NAD}^{\circ}+\mathrm{H}_{2} \mathrm{O} \\
& \mathrm{H}^{+}+\mathrm{O}_{2}^{-}+\mathrm{NADH} \rightarrow \mathrm{H}_{2} \mathrm{O}_{2}+\mathrm{NAD}^{\circ} \\
& \text { CoIII } \rightarrow \text { CoII }+\mathrm{H}_{2} \mathrm{O}_{2}
\end{aligned}
$$




$$
\begin{aligned}
& \underline{B F S O \text { Model }} \\
& \mathrm{O}_{2}^{-}+\mathrm{NADH}+\mathrm{H}^{+} \rightarrow \mathrm{NAD}^{-}+\mathrm{H}_{2} \mathrm{O}_{2} \\
& 2 \mathrm{O}_{2}^{-}+2 \mathrm{H}^{+} \rightarrow \mathrm{O}_{2}+\mathrm{H}_{2} \mathrm{O}_{2} \\
& 2 \mathrm{NAD}^{\circ} \rightarrow \quad(\mathrm{NAD})_{2} \\
& \mathrm{Per}^{3+}+\mathrm{NAD}^{\cdot} \rightarrow \mathrm{Per}^{2+}+\mathrm{NAD}^{+} \\
& \mathrm{Per}^{2+}+\mathrm{O}_{2} \rightarrow \mathrm{CoIII} \\
& \mathrm{NADH} \text { (stock) } \rightarrow \quad \mathrm{NADH} \text { (liquid) } \\
& \mathrm{O}_{2} \text { (gas) } \rightleftharpoons \quad \mathrm{O}_{2} \text { (liquid) } \\
& \mathrm{O}_{2}^{-}+\mathrm{NADH} \rightarrow \mathrm{NAD}^{-}+\mathrm{H}_{2} \mathrm{O}_{2} \\
& \rightarrow \quad \mathrm{O}_{2} \\
& \mathrm{NAD}^{\bullet} \rightarrow \\
& 2 \mathrm{O}_{2}^{-}+2 \mathrm{H}^{+} \rightarrow \mathrm{O}_{2}+\mathrm{H}_{2} \mathrm{O}_{2} \\
& \mathrm{NADH}_{\mathrm{R}} \rightarrow \quad \mathrm{NADH} \\
& \mathrm{NADH} \rightarrow \mathrm{NADH}_{\mathrm{P}} \text { (products) } \\
& \mathrm{O}_{2(\mathrm{~g})} \rightarrow \quad \mathrm{O}_{2(\mathrm{aq})} \\
& \mathrm{O}_{2(\mathrm{aq})} \rightarrow \quad \mathrm{O}_{2(\mathrm{~g})} \\
& 2 \mathrm{NAD}^{\circ} \rightarrow \quad(\mathrm{NAD})_{2}
\end{aligned}
$$




\subsection{CHEMICAL WAVES IN THE PEROXIDASE-OXIDASE REACTION}

Waves in the BZ reaction [61-63] were first reported by Zaikin and Zhabotinsky [7]. Since then, waves in the excitable BZ reaction have been extensively studied as a chemical model for waves in excitable biological media, such as heart tissue, brain tissues, and neuronal tissue [64-66].

The PO reaction, on the other hand, is more relevant for studying excitable biological media, as the PO reaction is known to occur in vivo. It occurs in plants during lignification of cell walls $[34,67]$. Peroxidase enzymes are important in mammals as well, and are often used for medical diagnosis $[68,34]$. The PO reaction represents a crucial link between the purely chemical oscillators and the purely biological oscillators, and its study will provide insights into the spatiotemporal behavior of biological systems. Though the experimental and theoretical understanding of this system has grown over the years, no studies of the nonlinear spatiotemporal behavior have been carried out on this system to date. The PO reaction satisfies the dynamical conditions necessary for the appearance of chemical waves and other spatial structures, and should thus be capable of sustaining chemical waves as in the $\mathrm{BZ}$ reaction. In the following section we present the results of one-dimensional numerical simulations of the BFSO-14 model that characterizes the nonlinear behavior of the PO reaction. We show that chemical waves can indeed be obtained in an oscillatory PO model system reaction after applying an external perturbation to system. 


\subsubsection{Generation of Chemical Waves in the BFSO-14 Model}

To study the generation of chemical waves an oscillatory PO reaction, numerical calculations were performed using an extended BFSO model. This model, however, is computationally intensive and more complicated when compared to the 1983 Olsen model and involves estimations of some rate constants. It was chosen over other models, however, as it is the more experimentally realistic model and better represents the nonlinear dynamics occurring in the PO reaction.

\subsubsection{The Chemical Reaction Model}

The chemical reactions comprising the extended BFSO model are

$$
\begin{aligned}
& \mathrm{O}_{2}+\mathrm{NADH}+\mathrm{H}^{+} \stackrel{\mathrm{k}_{1}}{\longrightarrow} \mathrm{NAD}^{+}+\mathrm{H}_{2} \mathrm{O}_{2} \\
& \mathrm{Per}^{3+}+\mathrm{H}_{2} \mathrm{O}_{2} \stackrel{\mathrm{k}_{2}}{\longrightarrow} \mathrm{CoI} \\
& \mathrm{CoI}+\mathrm{NADH} \stackrel{\mathrm{k}_{3}}{\longrightarrow} \mathrm{CoII}+\mathrm{NAD}^{\bullet} \\
& \mathrm{CoII}+\mathrm{NADH} \stackrel{\mathrm{k}_{4}}{\longrightarrow} \mathrm{Per}^{3+}+\mathrm{NAD}^{\bullet} \\
& \mathrm{NAD}^{\bullet}+\mathrm{O}_{2} \stackrel{\mathrm{k}_{5}}{\longrightarrow} \mathrm{NAD}^{+}+\mathrm{O}_{2}^{-} \\
& \mathrm{Per}^{3+}+\mathrm{O}_{2}^{-} \quad \stackrel{\mathrm{k}_{6}}{\longrightarrow} \text { CoIII } \\
& 2 \mathrm{O}_{2}^{-}+2 \mathrm{H}^{+} \stackrel{\mathrm{k}_{7}}{\longrightarrow} \mathrm{O}_{2}+\mathrm{H}_{2} \mathrm{O}_{2} \\
& \mathrm{CoIII}+\mathrm{NAD}^{\cdot} \stackrel{\mathrm{k}_{8}}{\longrightarrow} \mathrm{CoI}+\mathrm{NAD}^{+} \\
& 2 \mathrm{NAD}^{\bullet} \stackrel{\mathrm{k}_{9}}{\longrightarrow}(\mathrm{NAD})_{2} \\
& \mathrm{Per}^{3+}+\mathrm{NAD}^{\cdot} \stackrel{\mathrm{k}_{10}}{\longrightarrow} \mathrm{Per}^{2+}+\mathrm{NAD}^{+} \\
& \mathrm{Per}^{2+}+\mathrm{O}_{2} \stackrel{\mathrm{k}_{11}}{\longrightarrow} \text { CoIII }
\end{aligned}
$$




$$
\begin{array}{llll} 
& \mathrm{NADH} \text { (stock soln) } & \stackrel{\mathrm{k}_{12}}{\longrightarrow} & \mathrm{NADH}(\text { soln }) \\
\mathrm{O}_{2} \text { (gas) } & \stackrel{\mathrm{k}_{13}}{\longrightarrow} \mathrm{O}_{2}(\text { soln }) \\
\mathrm{O}_{2}^{-}+\mathrm{NADH}+\mathrm{H}^{+} \stackrel{\mathrm{k}_{14}}{\longrightarrow} \mathrm{H}_{2} \mathrm{O}_{2}+\mathrm{NAD}^{\circ}
\end{array}
$$

$\mathrm{MB}$ is incorporated into the rate constant of reaction (R19) as it is known to catalyze that reaction. The peroxidase cycle is represented by the three reactions $(\mathrm{R} 1)-(\mathrm{R} 3)$. Reactions (R24) and (R25) represent the continuous supplies of NADH and oxygen, respectively. The ordinary differential equations for the extended BFSO model are

$$
\begin{aligned}
& \mathrm{d}[\mathrm{NADH}] / \mathrm{dt}=-\mathrm{k}_{1} \mathrm{X}_{1} \mathrm{X}_{2}-\mathrm{k}_{3} \mathrm{X}_{5} \mathrm{X}_{1}-\mathrm{k}_{4} \mathrm{X}_{6} \mathrm{X}_{1}+\mathrm{k}_{12}[\mathrm{NADH}]_{\mathrm{st}}-\mathrm{k}_{14} \mathrm{X}_{1} \mathrm{X}_{9} \\
& \mathrm{~d}\left[\mathrm{O}_{2}\right]=-\mathrm{k}_{1} \mathrm{X}_{1} \mathrm{X}_{2}-\mathrm{k}_{5} \mathrm{X}_{3} \mathrm{X}_{2}+\mathrm{k}_{7}\left(\mathrm{X}_{9}\right)^{2}+\mathrm{k}_{13}\left[\mathrm{O}_{2}\right]_{\mathrm{gas}}-\mathrm{k}-{ }_{13} \mathrm{X}_{2}-\mathrm{k}_{11} \mathrm{X}_{10} \mathrm{X}_{2} \\
& \mathrm{~d}\left[\mathrm{NAD}^{\circ}\right]=\mathrm{k}_{3} \mathrm{X}_{5} \mathrm{X}_{1}+\mathrm{k}_{4} \mathrm{X}_{6} \mathrm{X}_{1}-\mathrm{k}_{5} \mathrm{X}_{3} \mathrm{X}_{2}-\mathrm{k}_{8} \mathrm{X}_{7} \mathrm{X}_{3}-2 \mathrm{k}_{9}\left(\mathrm{X}_{3}\right)^{2}-\mathrm{k}_{10} \mathrm{X}_{4} \mathrm{X}_{3}+\mathrm{k}_{14} \mathrm{X}_{1} \mathrm{X}_{9} \\
& \mathrm{~d}\left[\mathrm{Per}^{3+}\right]=-\mathrm{k}_{2} \mathrm{X}_{8} \mathrm{X}_{4}+\mathrm{k}_{4} \mathrm{X}_{6} \mathrm{X}_{1}-\mathrm{k}_{6} \mathrm{X}_{9} \mathrm{X}_{4}-\mathrm{k}_{10} \mathrm{X}_{4} \mathrm{X}_{3} \\
& \mathrm{~d}[\mathrm{CoI}]=\mathrm{k}_{2} \mathrm{X}_{8} \mathrm{X}_{4}-\mathrm{k}_{3} \mathrm{X}_{5} \mathrm{X}_{1}+\mathrm{k}_{8} \mathrm{X}_{7} \mathrm{X}_{3} \\
& \mathrm{~d}[\mathrm{CoII}]=\mathrm{k}_{3} \mathrm{X}_{5} \mathrm{X}_{1}-\mathrm{k}_{4} \mathrm{X}_{6} \mathrm{X}_{1} \\
& \mathrm{~d}[\mathrm{CoIII}]=\mathrm{k}_{6} \mathrm{X}_{9} \mathrm{X}_{4}-\mathrm{k}_{8} \mathrm{X}_{7} \mathrm{X}_{3}+\mathrm{k}_{11} \mathrm{X}_{10} \mathrm{X}_{2} \\
& \mathrm{~d}\left[\mathrm{H}_{2} \mathrm{O}_{2}\right]=\mathrm{k}_{1} \mathrm{X}_{1} \mathrm{X}_{2}-\mathrm{k}_{2} \mathrm{X}_{8} \mathrm{X}_{4}+\mathrm{k}_{7}\left(\mathrm{X}_{9}\right)^{2}+\mathrm{k}_{14} \mathrm{X}_{1} \mathrm{X}_{9} \\
& \mathrm{~d}\left[\mathrm{O}_{2}^{-}\right]=\mathrm{k}_{5} \mathrm{X}_{3} \mathrm{X}_{2}-\mathrm{k}_{6} \mathrm{X}_{9} \mathrm{X}_{4}-2 \mathrm{k}_{7}\left(\mathrm{X}_{9}\right)^{2}-\mathrm{k}_{14} \mathrm{X}_{1} \mathrm{X}_{9} \\
& \mathrm{~d}\left[\mathrm{Per}^{2+}\right]=\mathrm{k}_{10} \mathrm{X}_{4} \mathrm{X}_{3}-\mathrm{k}_{11} \mathrm{X}_{10} \mathrm{X}_{2}
\end{aligned}
$$

where $\mathrm{X}_{1}=\mathrm{NADH}, \mathrm{X}_{2}=\mathrm{O}_{2}, \mathrm{X}_{3}=\mathrm{NAD}^{\circ}, \mathrm{X}_{4}=\mathrm{Per}^{3+}, \mathrm{X}_{5}=\mathrm{CoI}, \mathrm{X}_{6}=\mathrm{CoII}, \mathrm{X}_{7}=$ CoIII, $\mathrm{X}_{8}$ $=\mathrm{H}_{2} \mathrm{O}_{2}, \mathrm{X}_{9}=\mathrm{O}_{2}^{-}$, and $\mathrm{X}_{10}=\mathrm{Per}^{2+}$. No attempt was made to transform the system into 
dimensionless equations and the parameters were used as given in Figure 8.1a without scaling.

\subsubsection{Reaction-Diffusion Equations for BFSO-14 Model}

Ten reaction-diffusion equations, obtained from the model that describes the evolution of ten variables in time, were numerically integrated after adding diffusion terms. Since the HRP enzyme will be immobilized in a gel matrix, the corresponding diffusion terms for the native form $\left(\mathrm{Per}^{3+}\right)$ and the four intermediates $\left(\mathrm{Per}^{2+}, \mathrm{CoI}, \mathrm{CoII}\right.$, and CoIII) were set to zero, $\mathrm{D}_{\mathrm{xx}}=0.0$. Although only numerical results are presented in this discussion, the actual experimental setup of the system will involve immobilizing the $\mathrm{HRP}$ in a gel matrix while the other variable species (NADH, $\mathrm{O}_{2}, \mathrm{NAD}^{\circ}, \mathrm{H}_{2} \mathrm{O}_{2}$, and $\mathrm{O}_{2}{ }^{-}$) are allowed to diffuse freely within the reactor.

\subsubsection{Wave Generation in the One-Dimensional BFSO-14 system}

We have performed one-dimensional numerical simulations of Eqs. (8.15) using the explicit Euler method with zero-flux boundary conditions and a three-point approximation for the Laplacian. Since simulations are performed for a one-dimensional configuration, the Laplacian becomes

$$
\nabla^{2}=\frac{\partial^{2}}{\partial x^{2}}
$$

A system size of 500 grid elements and a perturbation area of 40 grid elements were chosen for the simulations. To initiate chemical waves, an oscillatory PO system was 
perturbed at the center of the domain after a period of $100.0 \mathrm{~s}$ by changing the variable $\mathrm{NADH}$ for a period of $1.0 \mathrm{~s}$ according to

$$
\left[\mathrm{X}_{1}\right]_{\mathrm{p}}=\left[\mathrm{X}_{1}\right]+\left[\mathrm{X}_{1}\right]^{* \mathrm{x}}
$$

where $\left[\mathrm{X}_{1}\right]$ represents the concentration of NADH at the time of perturbation, and $\mathrm{x}$ is the fractional change. Figure 8.1a shows the resultant space-time plot with profiles of the $\mathrm{NAD}^{\circ}$ radical concentration (the autocatalyst in the PO system) at equal increments of time. The space-time plot demonstrates the initiation of trigger waves that propagate outwards in the medium. Unlike in an excitable system where trigger waves propagate from the pacemaker region to the boundary, in an oscillatory system the trigger wave propagates outwards to a point determined by the oscillations of the bulk system. The trigger wave disappears with each bulk oscillation, although it propagates farther and farther with each bulk oscillation. This is in agreement with studies by Ortoleva and Ross [15], which demonstrated that waves persisted longer with each bulk oscillation. Figure $8.1 \mathrm{~b}$ shows space-time plots taken after a period of 3500.0 seconds, with trigger waves propagating to the boundary. Initiation of trigger waves was possible using a perturbation of [NADH] with $1 \% \leq \mathrm{x} \leq 10 \%$. Perturbations in [NADH] of less than $1 \%$ did not initiate chemical waves.

To prove that the waves initiated in Figure 8.1 were trigger waves and not phase waves another calculation was carried out in which all parameters were the same as in Figure 8.1, except the diffusion coefficients were set to zero for all variables $\left(D_{x x}=0\right)$. Trigger waves, unlike phase waves, are blocked by barriers to diffusion. Hence, if the 
calculation produces the same results as in Figure 8.1, we can conclude that the waves observed are phase waves and not trigger waves. The calculations showed that no propagating waves appeared when all diffusion coefficients were set to zero, as shown in Figure 8.2. Instead, we see the region of perturbation oscillating at the same frequency as the bulk oscillations but out of phase for as long as the calculation is carried out. This shows that the waves in Figure 8.1 are trigger waves, as they require diffusion for their propagation.

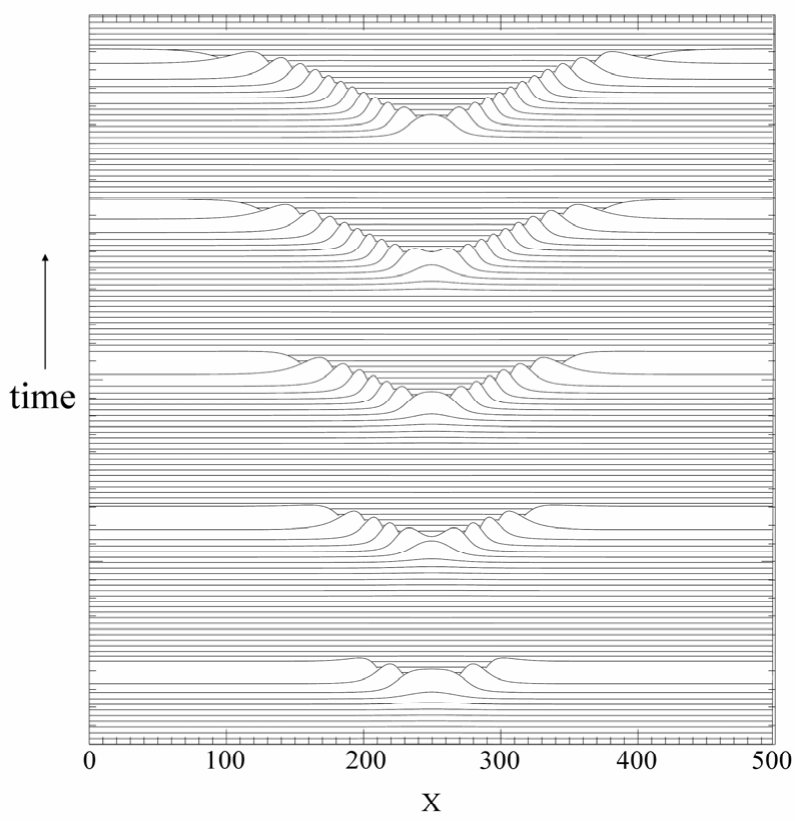

Figure 8.1a: Calculated space-time plots showing concentration profiles of the NAD radical. Perturbation of NADH (3\%) results in the initiation of a trigger wave which propagates outward before the whole medium oscillates. The trigger wave travels farther and farther with each bulk oscillation until it reaches the boundary. Profiles were overlayed in increments of 3.0 seconds, and, owing to the large period of the bulk oscillation (240.0 s), a period of 156 seconds was removed between the bulk oscillations. Model parameters of the system are given by $[16,17,18]$ : $\mathrm{k}_{1}=3.0, \mathrm{k}_{2}=1.8 \times 10^{7}, \mathrm{k}_{3}=4.0 \times 10^{4}, \mathrm{k}_{4}=2.6 \times 10^{4}, \mathrm{k}_{5}=2.0 \times 10^{7}, \mathrm{k}_{6}=1.7 \times 10^{7}, \mathrm{k}_{7}=2.0 \times 10^{7}$, $\mathrm{k}_{8}=1.0, \mathrm{k}_{9}=5.6 \times 10^{7}, \mathrm{k}_{10}=1.8 \times 10^{6}, \mathrm{k}_{11}=1.0 \times 10^{5}$ and $\mathrm{k}_{14}=3.0 \times 10^{2}$, all in $\mathrm{M}^{-1} \mathrm{~s}^{-1}$. The rate of reaction (R24) $\left(\mathrm{k}_{12}[\mathrm{NADH}]_{\mathrm{st}}\right)$ was $1.14314 \times 10^{-7} \mathrm{Ms}^{-1}$, the first order rate constant $\mathrm{k}_{-13}$ was equal to $3.73 \times 10^{-3} \mathrm{~s}^{-1}$, and the rate of diffusion of oxygen into the reactor $\left(\mathrm{k}_{13}\left[\mathrm{O}_{2}\right]_{\text {gas }}\right)$ was $6.2415 \times 10^{-8}$ $\mathrm{Ms}^{-1}$. Initial conditions: $\mathrm{X}_{1}=747.049395 \mu \mathrm{M}, \mathrm{X}_{2}=4.580033 \mu \mathrm{M}, \mathrm{X}_{3}=0.001125 \mu \mathrm{M}, \mathrm{X}_{4}=$ $0.001109 \mu \mathrm{M}, \mathrm{X}_{5}=0.001566 \mu \mathrm{M}, \mathrm{X}_{6}=0.002407 \mu \mathrm{M}, \mathrm{X}_{7}=0.894912 \mu \mathrm{M}, \mathrm{X}_{8}=2.296717 \mu \mathrm{M}, \mathrm{X}_{9}$ $=0.047679 \mu \mathrm{M}$, and $\mathrm{X}_{10}=0.000004 \mu \mathrm{M}$. Diffusion parameters: $\mathrm{D}_{\mathrm{X} 4}=\mathrm{D}_{\mathrm{X} 5}=\mathrm{D}_{\mathrm{X} 6}=\mathrm{D}_{\mathrm{X} 7}=\mathrm{DX}_{10}=$ $0.0, \mathrm{D}_{\mathrm{X} 1}=4.0 \times 10^{-5}, \mathrm{D}_{\mathrm{X} 2}=8.2 \times 10^{-4}, \mathrm{D}_{\mathrm{X} 3}=4.0 \times 10^{-5}, \mathrm{D}_{\mathrm{X} 8}=7.8 \times 10^{-4}$, and $\mathrm{D}_{\mathrm{X} 9}=8.2 \times 10^{-4}$ all in $\mathrm{cm}^{2} \mathrm{~s}^{-1}$. The simulation was carried out using a one-dimensional lattice of 500 grid points $(\Delta x=$ $0.02, \Delta t=0.0001)$. 


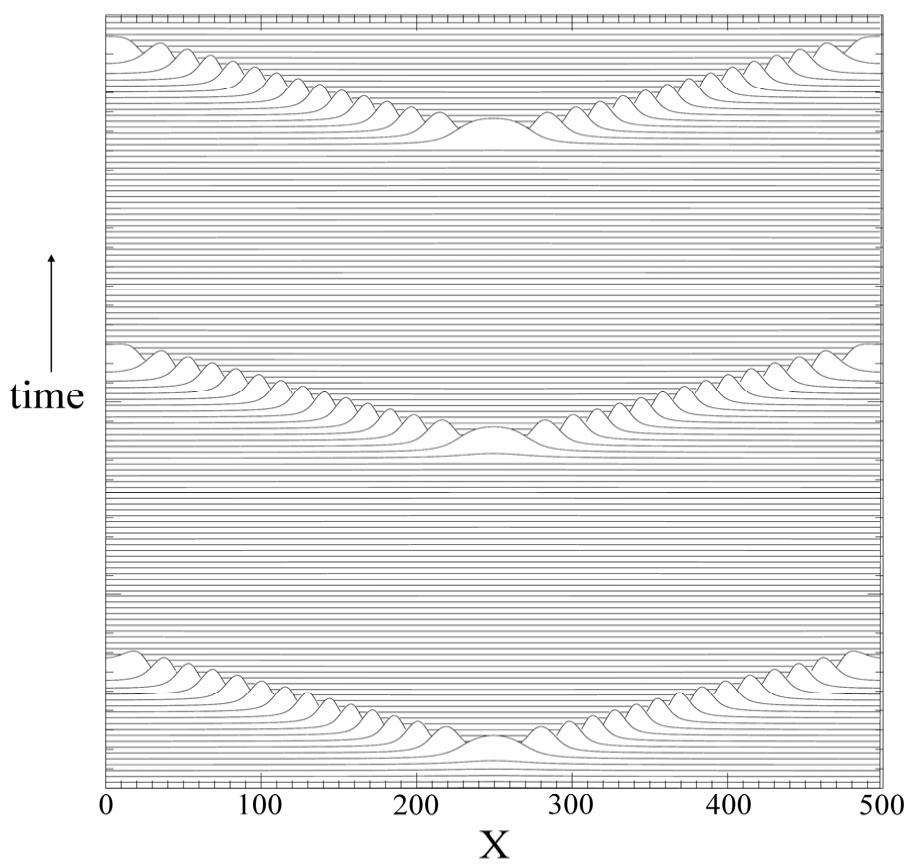

Figure 8.1b: Space-time plots showing concentration profiles of the NAD ${ }^{\circ}$ radical demonstrating wave propagation to the boundary. Model parameters are the same as in Figure 8.1 except that profiles are taken $3500.0 \mathrm{~s}$ into the reaction. Profiles were overlayed in increments of 4.0 seconds, and owing to the large period of the bulk oscillations $(240.0 \mathrm{~s})$ a period of 20.0 seconds was removed between the oscillations.

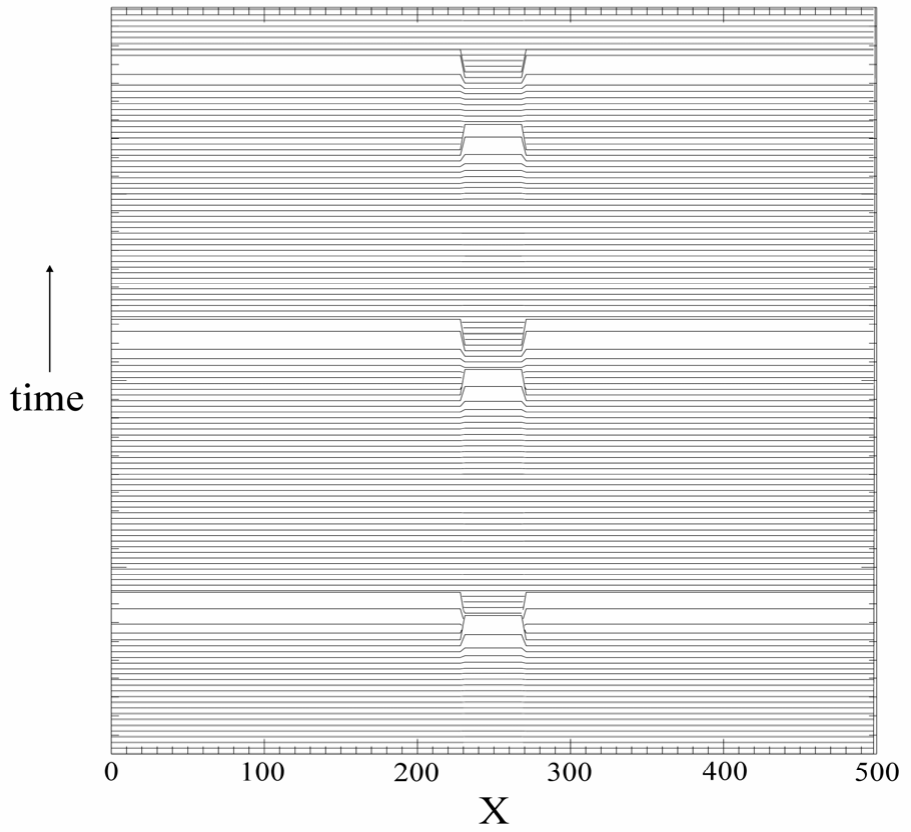

Figure 8.2: Space-time plots showing profiles of NAD ${ }^{\circ}$ radical concentration. All model parameters are the same as in Figure 8.1 except that all diffusion coefficients are set to zero. No trigger wave is initiated in this case. Profiles were overlayed in increments of 2.0 seconds, and owing to the large period of the bulk oscillations $(240.0 \mathrm{~s})$ a period of 140.0 seconds was removed between the bulk oscillations. 


\subsection{DISCUSSION AND CONCLUSION}

In addition to a review of the PO reaction, we have been able to demonstrate the initiation of traveling waves in the extended BFSO model. One-dimensional simulations in the BFSO-14 model have revealed that trigger waves will indeed propagate in an oscillatory Peroxidase-Oxidase system. The fact that the chemical waves had a characteristic sharp front and were not able to penetrate barriers to diffusion demonstrates that the waves were trigger waves. Phase waves, on the other hand, generally have broader fronts [13] and are able to penetrate barriers to diffusion, as they do not depend on diffusion for their propagation.

The numerical results presented here suggest the likelihood of experimentally observing wave propagation in the PO reaction. As far as we know, our study constitutes the first example of the generation of chemical waves in a PO reaction model system. 


\section{References}

1. R. Kapral and K. Showalter, Chemical Waves and Patterns, (Kluwer Academic Publishers, Boston, 1995).

2. I. R. Epstein and K. Showalter, "Nonlinear chemical dynamics: Oscillations, patterns, and chaos," J. Phys. Chem. 100, 13132-13147 (1996).

3. A. T. Winfree, "Electrical turbulence in three-dimensional heart muscle," Science 266, 1003-1006 (1994).

4. P. Jung, A. Cornell-Bell, K. S. Madden, and F. Moss, "Noise-induced spiral waves in Astrocyte syncytia show evidence of self-organized criticality," J. Neurophysiol. 79, 1098-1101 (1998).

5. J. Beaumont, N. Davidenko, J. M. Davidenko, and J. Jalife, "Spiral waves in twodimensional models of ventricular muscle: Formation of a stationary core," Biophys. J. 75, 1-14 (1998).

6. S. C. Mueller, T. Plesser, A. Boiteux, and B. Hess, "Pattern formation and Marangoni convection during oscillating glycolysis," J. Biosci. 40C, 588-591 (1985).

7. A. N. Zaikin and A. M. Zhabotinsky, "Concentration wave propagation in twodimensional liquid-phase self oscillating system," Nature 225, 535-537 (1970).

8. J. Ross, S. C. Muller, and C. Vidal, "Chemical Waves," Science 240, 460-465 (1988).

9. E. J. Reusser and R. J. Field, "The transition from phase waves to trigger waves in a model of the Zhabotinskii reaction," J. Am. Chem. Soc. 101, 1063-1071 (1979).

10. R. R. Aliev, "Oscillation phase dynamics in the Belousov-Zhabotinsky reaction. Implementation to image processing," J. Phys. Chem. 98, 3999-4002 (1994).

11. R. R. Aliev and V. N. Biktashev, "Dynamics of the oscillation phase distribution in the BZ reaction," J. Phys. Chem. 98, 9676-9681 (1994).

12. R. R. Aliev, V. A. Davydov, T. Ohmori, M. Nakaiwa, and T. Yamaguchi, "Change of the shape of a chemical vortex due to a local disturbance," J. Phys. Chem. A 101, 1313-1316 (1997).

13. J. M. Bodet, J. Ross, and C. Vidal, "Experiments on phase diffusion waves," J. Chem. Phys. 86, 4418-4424 (1987).

14. Y. X. Zhang, P. Foerster, and J. Ross, "Origin of spontaneous wave generation in an oscillatory chemical system," J. Phys. Chem. 96, 8898-8904 (1992). 
15. P. Ortoleva and J. Ross, "Phase waves in oscillatory chemical reactions," J. Chem. Phys. 58, 5673-5680 (1973).

16. T. V. Bronnikova, V. R. Fed'kina, W. M. Schaffer, and L. F. Olsen, "Perioddoubling bifurcations and chaos in a detailed model of the peroxidase-oxidase reaction," J. Phys. Chem. 99, 9309-9312 (1995).

17. T. V. Bronnikova, W. M. Schaffer, and L. F. Olsen, "Nonlinear dynamics of the peroxidase-oxidase reaction: I. Bistability and bursting oscillations at low enzyme concentrations," J. Phys. Chem. B 105, 310-321 (2001).

18. W. M. Schaffer, T. V. Bronnikova, and L. F. Olsen, "Nonlinear dynamics of the peroxidase-oxidase reaction. II. Compatibility of an extended model with previously reported model-data correspondences," J. Phys. Chem. B 105, 5331$5340(2001)$.

19. I. Yamazaki, K. Yokota, and R. Nakajima, "Oscillatory oxidations of reduced pyridine nucleotide by peroxidase," Biochem. Biophys. Res. Commun. 21, 582586 (1965).

20. K. Yokota and I. Yamazaki, "Reaction of peroxidase with reduced nicotinamideadenine dinucleotide and reduced nicotinamide-adenine dinucleotide phosphate," Biochim. Biophys. Acta 105, 301-312 (1965).

21. S. Nakamura, K. Yokota, and I. Yamazaki, "Sustained oscillations in a lactoperoxidase. NADPH and $\mathrm{O}_{2}$ system," Nature 222, 794 (1969).

22. K. R. Valeur and L. F. Olsen, "Kinetic studies of the oscillatory dynamics in the peroxidase-oxidase reaction catalyzed by four different peroxidases," Biochim. Biophys. Acta 1289, 377-384 (1996).

23. U. Kummer, K. R. Valeur, G. Baier, K. Wegmann, and L. F. Olsen, "Oscillations in the peroxidase-oxidase reaction: A comparison of different peroxidases," Biochim. Biophys. Acta 1289, 397-403 (1996).

24. H. Degn, "Compound 3 kinetics and chemiluminescence in oscillatory oxidation reactions catalyzed by horseradish peroxidase," Biochim. Biophys. Acta 180, 271290 (1969).

25. M. J. B. Hauser and L. F. Olsen, "Mixed-mode oscillations and homoclinic chaos in an enzyme reaction," J. Chem. Soc. Faraday T. 92, 2857-2863 (1996).

26. T. Hauck and F. W. Schneider, "Mixed-mode and quasiperiodic oscillations in the peroxidase-oxidase reaction," J. Phys. Chem. 97, 391-397 (1993).

27. R. Larter and S. Hemkin, "Further refinements of the peroxidase-oxidase oscillator mechanism: Mixed-mode oscillations and chaos," J. Phys. Chem. 100, 18924-18930 (1996). 
28. B. D. Aguda, L. L. Hofmann Frisch, and L. Folke Olsen, "Experimental evidence for the coexistence of oscillatory and steady states in the peroxidase-oxidase reaction," J. Am. Chem. Soc. 112, 6652-6656 (1990).

29. M. S. Samples, Y. F. Hung, and J. Ross, "Further experimental studies on the horseradish peroxidase-oxidase reaction," J. Phys. Chem. 96, 7338-7342 (1992).

30. M. J. B. Hauser, L. F. Olsen, T. V. Bronnikova, and W. M. Schaffer, "Routes to chaos in the peroxidase-oxidase reaction: Period-doubling and period-adding," J. Phys. Chem. B 101, 5075-5083 (1997).

31. T. Geest, C. G. Steinmetz, R. Larter, and L. F. Olsen, "Period-doubling bifurcations and chaos in an enzyme reaction," J. Phys. Chem. 96, 5678-5680 (1992).

32. T. Hauck and F. W. Schneider, "Chaos in a Farey sequence through period doubling in the peroxidase-oxidase reaction," J. Phys. Chem. 98, 2072-2077 (1994).

33. H. B. Dunford, Heme Peroxidases, (John Wiley, New York, 1999).

34. A. Scheeline, D. L. Olson, E. P. Williksen, G. A. Horras, M. L. Klein, and R. Larter, "The peroxidase-oxidase oscillator and its constituent chemistries," Chem. Rev. 97, 739-756 (1997).

35. I. Yamazaki and K. Yokota, "Oxidation states of peroxidase," Mol. Cell Biochem. 2, 39-52 (1973).

36. L. F. Olsen and H. Degn, "Oscillatory kinetics of the peroxidase-oxidase reaction in an open system. Experimental and theoretical studies," Biochim. Biophys. Acta 523, 321-334 (1978).

37. L. F. Olsen and H. Degn, "Chaos in an enzyme reaction," Nature 267, 177-178 (1977).

38. T. Y. Li and J. A. Yorke, "Period three implies chaos," Am. Math. Mon. 82, 985992 (1975).

39. R. A. Schmitz, K. R. Graziani, and J. L. Hudson, "Experimental evidence of chaotic states in the Belousov-Zhabotinsky reaction," J. Chem. Phys. 67, 30403044 (1977).

40. M. J. B. Hauser, A. Lunding, and L. Folke Olsen, "On the role of methylene blue in the oscillating peroxidase-oxidase reaction," Phys. Chem. Chem. Phys. 2, 1685-1692 (2000). 
41. D. L. Olson and A. Scheeline, "The peroxidase-NADH biochemical oscillator. 1. Examination of oxygen mass transport, the effect of light, and the role of methylene blue," J. Phys. Chem. 99, 1204-1211 (1995).

42. P. Sevcik and H. B. Dunford, "Kinetics of the oxidation of NADH by methylene blue in a closed system," J. Phys. Chem. 95, 2411-2415 (1991).

43. M. J. Hauser and L. F. Olsen, "The role of naturally occurring phenols in inducing oscillations in the peroxidase-oxidase reaction," Biochemistry 37, 2458-2469 (1998).

44. A. C. Moller, M. J. B. Hauser, and L. F. Olsen, "Oscillations in peroxidasecatalyzed reactions and their potential function in vivo," Biophys. Chem. 72, 6372 (1998).

45. U. Kummer, M. J. B. Hauser, K. Wegmann, L. F. Olsen, and Baier Gerold, "Oscillations and complex Dynamics in the peroxidase-oxidase reaction induced by naturally occurring aromatic substrates," J. Am. Chem. Soc. 119, 2084-2087 (1997).

46. H. Degn, "Bistability caused by substrate inhibition of peroxidase in an open reaction system," Nature 217, 1047-1050 (1968).

47. H. Degn, L. F. Olsen, and J. W. Perram, "Bistability, oscillation, and chaos in an enzyme reaction," Ann. N. Y. Acad. Sci. 316, 623-637 (1979).

48. B. D. Aguda and R. Larter, "Sustained oscillations and bistability in a detailed mechanism of the peroxidase-oxidase reaction," J. Am. Chem. Soc. 112, 21672174 (1990).

49. R. Larter, L. F. Olsen, C. G. Steinmetz, and T. Geest, "Chaos in biochemical stystems: The peroxidase reaction as a case study," in Chaos in Chemistry and Biochemistry, edited by R.J.Field and L.Gyorgyi, (World Scientific, Singapore 1993) p. 175-224.

50. R. Larter, C. G. Steinmetz, and B. D. Aguda, "Fast-slow variable analysis of the transition to mixed-mode oscillations and chaos in the peroxidase reaction," J. Chem. Phys. 89, 6506-6514 (1988).

51. R. Larter, C. L. Bush, T. R. Lonis, and B. D. Aguda, "Multiple steady states, complex oscillations, and the devil's staircase in the peroxidase-oxidase reaction," J. Chem. Phys. 87, 5765-5771 (1987).

52. L. F. Olsen, "An enzyme reaction with a strange attractor," Phys.Lett. A 94A, 454-457 (1983). 
53. B. D. Aguda, R. Larter, and B. L. Clarke, "Dynamic elements of mixed-mode oscillations and chaos in a peroxidase-oxidase model network," J. Chem. Phys. 90, 4168-4175 (1989).

54. S. Alexandre and H. B. Dunford, "A new model for oscillations in the peroxidaseoxidase reaction," Biophys. Chem. 40, 189-195 (1991).

55. K. Yokota and I. Yamazaki, "Analysis and computer simulation of aerobic oxidation of reduced nicotinamide adenine dinucleotide catalyzed by horseradish peroxidase," Biochemistry 16, 1913-1920 (1977).

56. V. R. Fed'kina, F. I. Ataullakhanov, and T. V. Bronnikova, "Computer simulation of sustained oscillations in peroxidase-oxidase reaction," Biophys. Chem. 19, 259-264 (1984).

57. B. D. Aguda and B. L. Clarke, "Bistability in chemical reaction networks: theory and application to the peroxidase-oxidase reaction," J. Chem. Phys. 87, 34613470 (1987).

58. B. D. Aguda and R. Larter, "Periodic-chaotic sequences in a detailed mechanism of the peroxidase-oxidase reaction," J. Am. Chem. Soc. 113, 7913-7916 (1991).

59. D. L. Olson, E. P. Williksen, and A. Scheeline, "An Experimentally based model of the Peroxidase-NADH biochemical oscillator: An enzyme-mediated chemical switch," J. Am. Chem. Soc. 117, 2-15 (1995).

60. A. C. Moller and L. F. Olsen, "Perturbations of simple oscillations and complex dynamics in the peroxidase-oxidase reaction using magnetic fields," J. Phys. Chem. B 104, 140-146 (2000).

61. A. M. Zhabotinsky, "Periodic process of the oxidation of malonic acid in solution (study of kinetics of Belousov's reaction)," Biofizika 9, 306-311 (1964).

62. R. M. Noyes and R. J. Field, "Oscillatory chemical reactions," Annu. Rev. Phys. Chem. 25, 95-119 (1974).

63. A. M. Zhabotinsky, Oscillatory Processes in Biological and Chemical Systems, (Science Publishers, Moscow, 1967).

64. A. T. Winfree, "Spiral waves of chemical activity," Science 175, 634-636 (1972).

65. K. Suzuki, T. Yoshinobu, and H. Iwasaki, "Induction of chemical waves by mechanical stimulation in elastic Belousov-Zhabotinsky media," Chem. Phys. Lett. 349, 437-441 (2001).

66. L. Kuhnert, K. I. Agladze, and V. I. Krinskii, "Image processing using lightsensitive chemical waves," Nature 337, 244-247 (1989). 
67. B. Halliwell, "Lignin synthesis: The generation of hydrogen peroxide and superoxide by horseradish peroxidase and its stimulation by manganese (II) and phenols.," Planta 140, 81-88 (1978).

68. B. Halliwell and J. M. Gutteridge, "Role of free radicals and catalytic metal ions in human disease: An overview," Methods Enzymol. 186,1-85 (1990). 